\title{
Synthesis and Characterization of Silicon and Germanium Nanocrystals and Titanium Disulphide Nanostructures
}

\author{
by
}

Sujay Prabakar

\author{
A thesis \\ Submitted to Victoria University of Wellington \\ in fulfillment of the \\ Requirements for the degree of \\ Doctor of Philosophy \\ in Chemistry
}

Victoria University of Wellington

June 2010 
(C) Copyright 2010

by

Sujay Prabakar

All Rights Reserved 
'Alchemy may be compared to the man who told his sons he had left the gold buried somewhere in his vineyard; where they by digging found no gold, but by turning up the mould, about the roots of their vines, procured a plentiful vintage. So the search and endeavors to make gold have brought many useful inventions and instructive experiments to light.'

\section{Francis Bacon}




\begin{abstract}
Keywords: Solution synthesis, silicon nanocrystals, germanium nanocrystals, size control, hydride reducing agents, fluorescent whitening agents, titanium disulphide nanostructures
\end{abstract}

This thesis is concerned with the synthesis and characterization of nanostructured materials in the solution, in particular silicon and germanium nanocrystals, their application as fluorescent whitening agents and titanium disulphide nanostructures. The aim of this research with regards to the synthesis of silicon and germanium nanocrystals was to obtain size control and provide functionality using simple room temperature solution techniques. In the case of the nanostructures of titanium disulphide, the focus was to synthesize in the colloid using simple one-pot bench top techniques. The above were realized with chemical techniques in the solution using organic solvents and surfactants to control their size. The morphology, chemical composition and crystal structure of the synthesized nanomaterials were characterized using techniques such as High Resolution Transmission Electron Microscopy (HRTEM), Selected Area Electron Diffraction (SAED), Scanning Electron Microscopy (SEM), Energy Dispersive X-Ray Spectroscopy (EDX) and Optical Spectroscopies. Whilst chapter one is a brief introduction of the thesis, chapter two talks in detail about the various characterization techniques used in this research.

Chapter three of the thesis focuses on the synthesis of alkyl- and amine-functionalized silicon nanocrystals using a microemlusion technique. The effect of reducing agents, 
surfactants and precursors on particle size was studied. The surfactant $\mathrm{C}_{12} \mathrm{E}_{5}$ was found to be very effective in producing silicon nanocrystals that were freestanding and pure. Whilst the hydride reducing agents lithium aluminium hydride and lithium tri-ethyl borohydride were found to be effective in synthesizing nanocrystals of narrow size distribution, it was found that using silicon tetrachloride yielded smaller particles compared to silicon tetrabromide.

The fourth chapter in the theses is concerned with the synthesis and characterization of germanium nanocrystals by both microemulsion and high temperature techniques. Using lithium aluminium hydride; a strong reducing agent, very small nanocrystals were obtained, whilst weaker reductants such as sodium borohydride produced larger nanocrystals. Another effective method to control the particle size of germanium nanocrystals was found to be by varying the concentration of precursor. The germanium nanocrystals which were amine capped were found to luminesce in the blue and were used to image HePG2 cells. Toxicity studies on these nanocrystals proved their relative non-toxicity. The high temperature experiments, though not as flexible as the room temperature syntheses were found to facilitate a certain degree of size control.

Chapter five of the theses deal with the application of silicon and germanium nanocrystals as fluorescent whitening agents in wool fabrics. Both nanocrystals, when applied to the fabric were found to emit matching blue fluorescence that was demonstrated to be more suited to improving the brightness properties of fabric than the commercial fluorescing whitening agent Uvitex. In particular Silicon-amine and Silicon-hexene functionalized nanocrystal (low concentration) treated fabrics were found to have

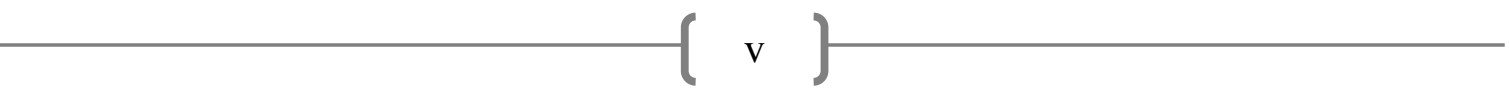


improved color stability against both UVA and UVB radiation. The treated fabrics were in addition found to maintain a stable color than untreated fabric. Silicon-amine treated fabrics were found to have a stable color even after $48 \mathrm{~h}$ exposures to UVA radiation. It should be noted that this is the first evidence of the application of group IV semiconductor nanocrystals as fluorescing whitening agents.

The sixth chapter of this thesis deals with the one-pot synthesis of titanium disulphide nanostructures using both coordinating and non-coordinating solvents and their subsequent characterization. By varying the injection temperature of the titanium source into the 1-Octadecene sulphur solution, two different morphologies were synthesized. Two different pathways were suggested for the formation of the flower-like and flake-like morphologies; an instant nucleation to form titanium disulphide flakes whilst spherical nuclei to form flower-like nanostructures. The flower-like nanostructures were found to have higher BET surface area compared to the flake-like nanostructures and previously reported surface areas for analogous $\mathrm{TiS}_{2}$ nanostructures. Whilst using oleylamine as solvent, the low temperature injection yielded hollow spheres of $\mathrm{TiS}_{2}$ and the high temperature injection, fullerene-like nanoparticles of $\mathrm{TiS}_{2}$. The property of oleylamine to selectively bind to the nanostructure surface in conjunction with the effect of injection temperature was understood to be behind the growth of these nanostructures. The synthesis of flower-like and flake-like morphologies by solution phase techniques were the first evidence of this kind for titanium disulphide and provides a new and exciting material for a variety of applications.

A final chapter on conclusions and recommendations for future work is then presented.

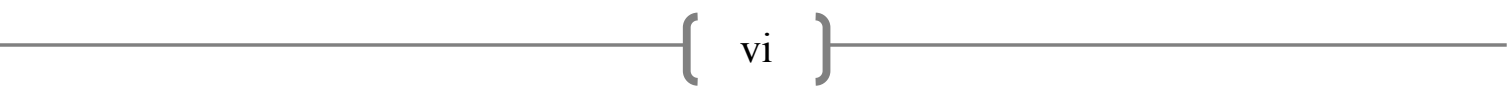




\section{Acknowledgements}

It is with great pleasure that I thank and acknowledge the many people who have contributed to my thesis as well as to my career and life over the past four years. I have had the good fortune to work with and learn from many talented people at Victoria University. I would like to thank in particular:

My supervisor Dr Richard Tilley for being an enthusiastic scientist, and for his direction in performing this research. I would also like to thank Richard for introducing me to the world of electron microscopy, and in particular, mentoring me in the art of writing good quality scientific articles.

My secondary supervisor Dr Shaun Hendy for his kind words and encouragement.

David Flynn for his help with the Electron Microscopes.

Dr. Chris Bumby for his exuberance and bright ideas during the silicon days, and also for introducing me to cricket at Karori.

Dr Jolon Dyer and Dr Santanu Deb Choudhury from AgResearch, Christchurch for being great hosts and helping with the FWA research.

Dr Paul Wilson for introducing me to the world of materials science research.

The Tilley Research Group for their scintillating discussions and constant support, in particular Soshan Cheong, John Watt and Amane Shiohara.

Both teaching and non-teaching staff at the School of Chemical and Physical Sciences for their cooperation and assistance; fellow students in the school for their help. 
Flatmates and friends Giorgi, Jay, Emily, Hoiran, Mattie, Josh and Bim for their help and company whilst at Waiteata. A special thanks to Jay for being a great mate during hard times.

Bumble and Vanitha akka, for their love and affection over the years. Lee, Rachel, Gary, Maria and Ethan for the fun times at Te Awamutu.

My uncle Victor for being a source of inspiration to undertake a career in science.

Mum, Dad and Gooch, who have been supportive from afar both financially and morally and have done their best to cheer me up or sympathize with me as I have struggled through a world quite foreign to them.

I would like to thank my wife Cathy, to whom for the past five years I have had the joy and privilege to share all of my successes and fears.

But above all it's God's grace that has led me all the way and I am what Iam because of the Almighty. 


\section{Table of Contents}

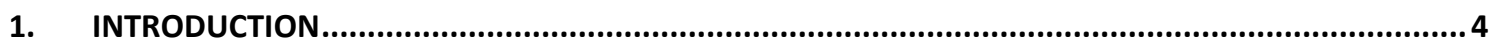

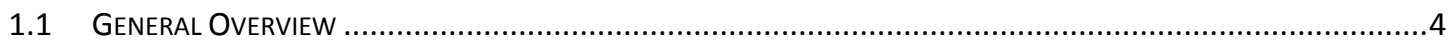

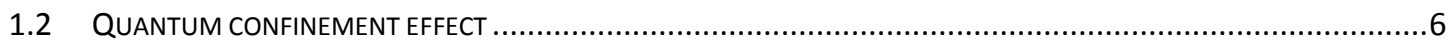

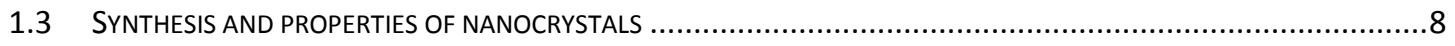

1.4 COLLOIDAL SYNTHESIS OF SEMICONDUCTOR QUANTUM DOTS...................................................................

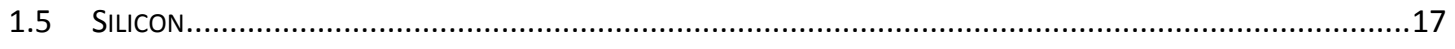

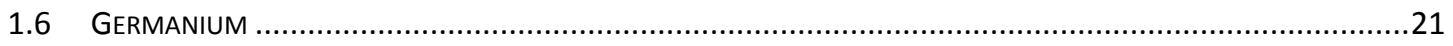

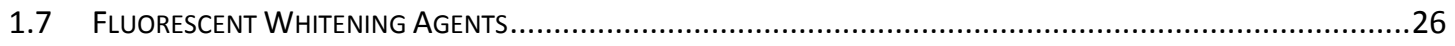

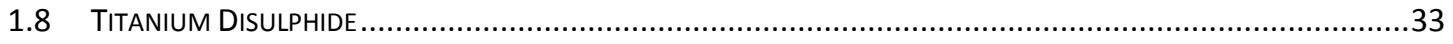

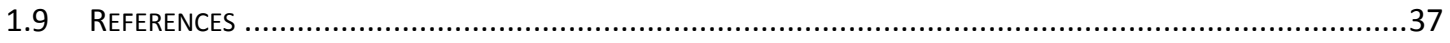

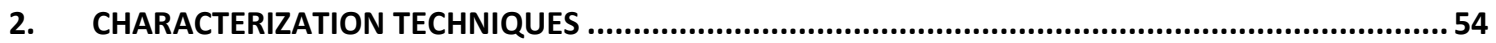

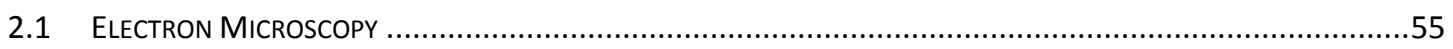

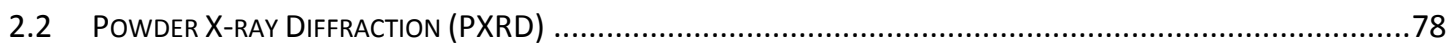

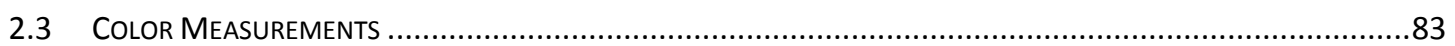

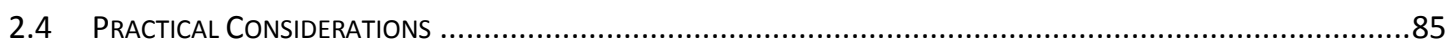

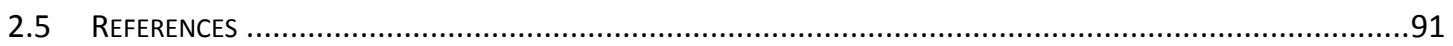

3. SOLUTION SYNTHESIS AND CHARACTERIZATION OF SILICON NANOCRYSTALS..........................93

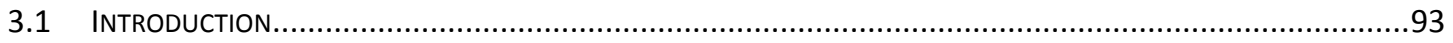

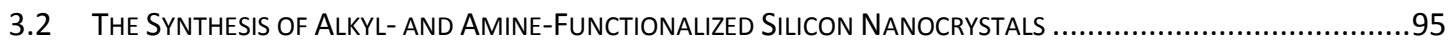

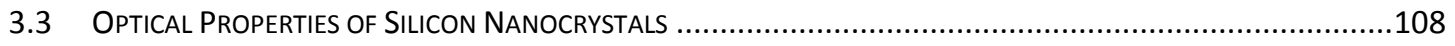

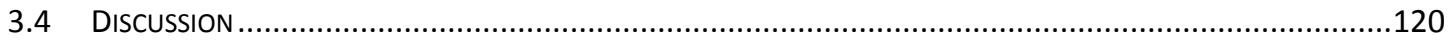

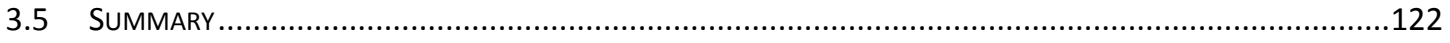

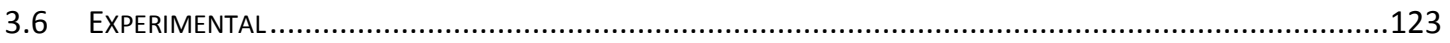

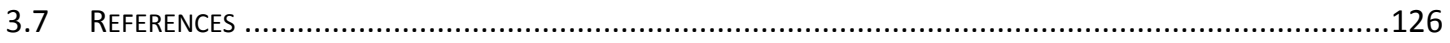

4. SOLUTION SYNTHESIS AND CHARACTERIZATION OF GERMANIUM NANOCRYSTALS ................. 131

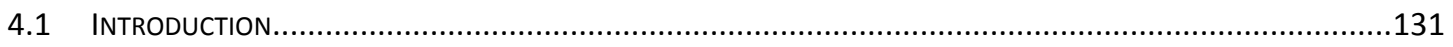

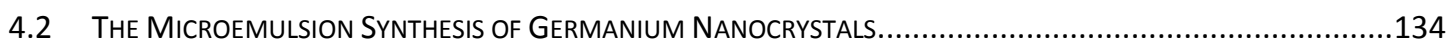

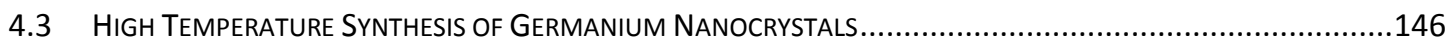

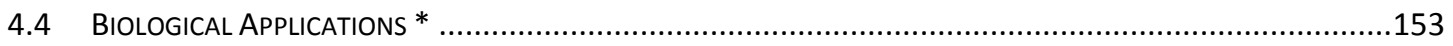

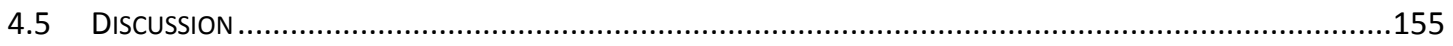

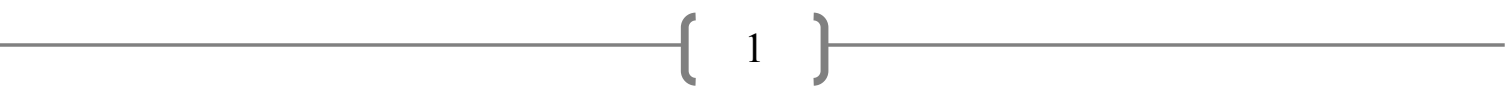




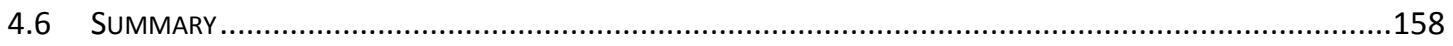

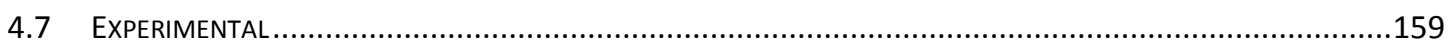

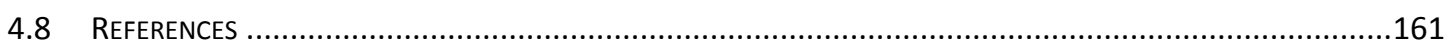

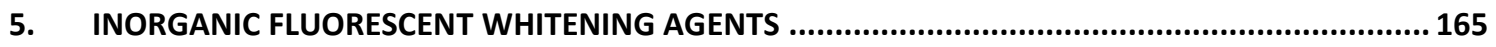

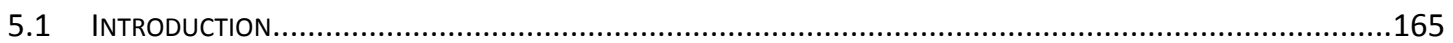

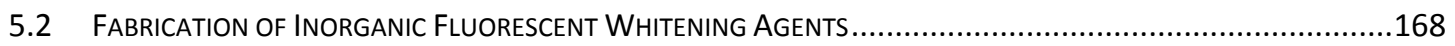

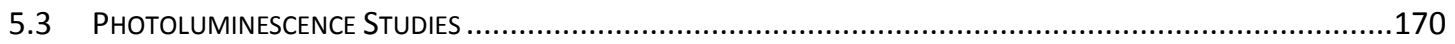

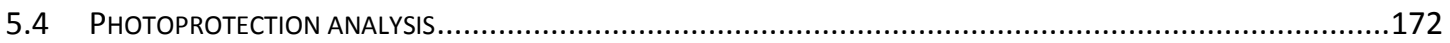

5.5 HPLC Assay EVAluating the OXIDATIVE Degradation of LUMINOL ............................................184

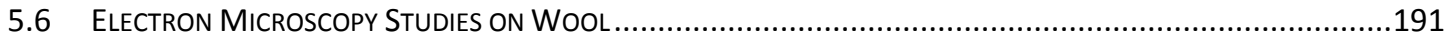

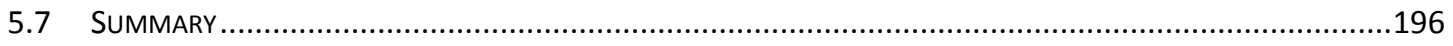

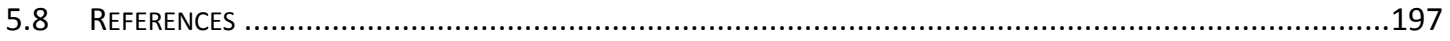

6. SOLUTION SYNTHESIS OF TITANIUM DISULPHIDE NANOSTRUCTURES ..................................200

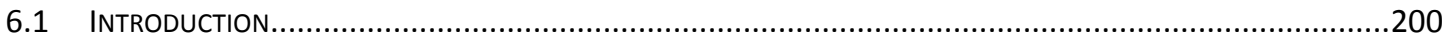

6.2 THE REACTION OF TITANIUM ISOPROPOXIDE AND ELEMENTAL SULFUR: THE FORMATION OF TITANIUM DIOXIDE

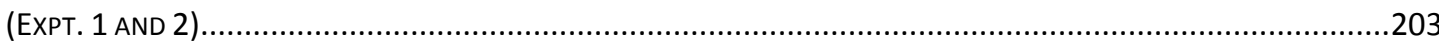

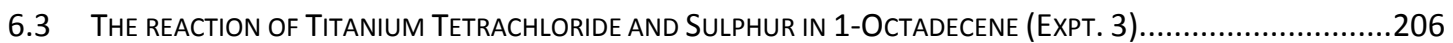

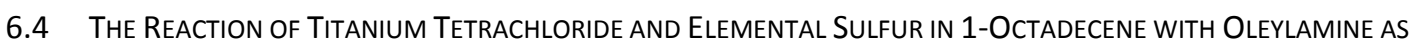

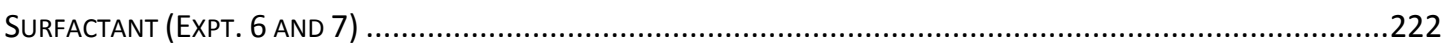

6.5 The Reaction of Titanium Tetrachloride And Elemental Sulfur In OleyLAMine (EXPT. 8 ANd 9) ........223

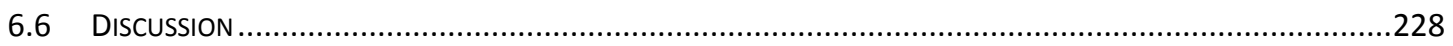

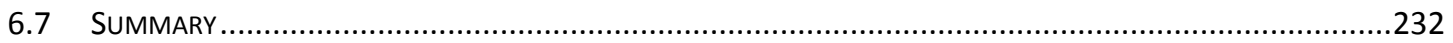

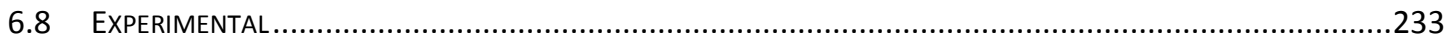

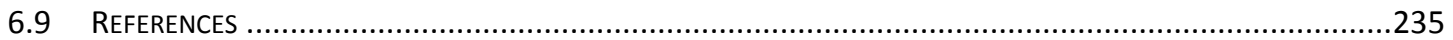

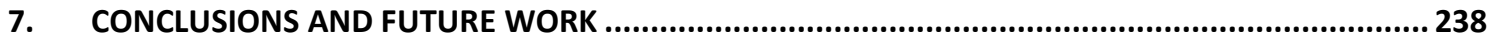

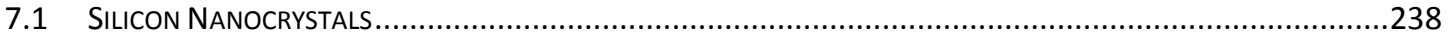

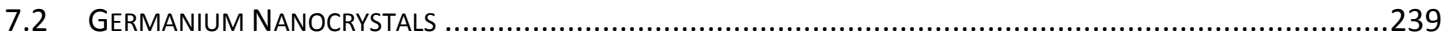

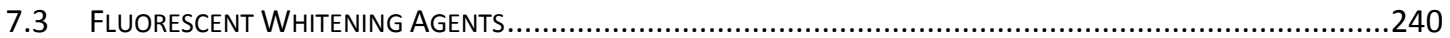

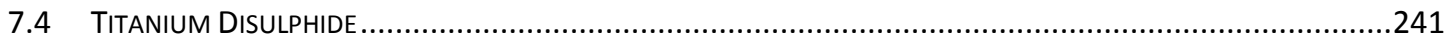

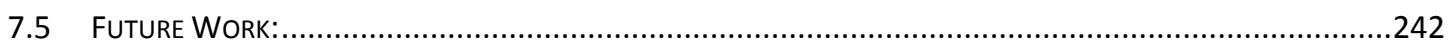

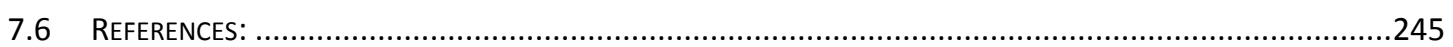




\begin{tabular}{ll} 
& \multicolumn{1}{c}{ Abbreviations } \\
ED & Electron Diffraction \\
EDS & Energy Dispersive X-ray Spectrometry \\
FESEM & Field Emission Scanning Electron Microscope \\
FFT & Fast Fourier Transform \\
HRTEM & High Resolution Transmission Electron Microscopy \\
h & hour \\
IR & Infra-red \\
PL & Photoluminescence \\
PXRD & Powder X-ray diffraction \\
SAED & Selected Area Electron Diffraction \\
SEM & Scanning Electron Microscopy \\
UV-Vis & Ultra-Violet-Visible \\
&
\end{tabular}




\section{Introduction}

\subsection{General Overview}

There has been considerable interest in the solution synthesis of nanocrystalline materials over the past few years due to advances in synthetic strategies and characterization techniques. When the size shrinks to the nanoscale, the large surface to volume ratio and quantum size effects of materials give rise to unique electrical and optical properties and can be used in diverse applications such as optoelectronics, sensing, catalysis and medicine. ${ }^{1-4}$

The synthesis of semiconductor nanocrystals by colloidal techniques has the advantage of providing control over size, shape, composition and surface chemistry. ${ }^{5-7}$ This facilitates the synthesis of easily dissolvable nanomaterials for low-temperature processing, deposition on plastic substrates, as additives in polymeric coatings and composites and biological applications. Colloidal techniques can produce narrow size and shape distributions of many kinds of nanometer size crystalline particles with stabilized surfaces and useful optical and electronic properties. ${ }^{8-12}$ While, the development of synthetic routes for the fabrication of colloidal semiconductor nanocrystals has paved the way for investigations into a variety of size-dependent optical and thermodynamic effects, the majority of those systems are II-IV semiconductors and much less attention has been paid to the technologically important group IV semiconductors, Silicon and Germanium. 
The research in this thesis deals with the synthesis of semiconductor nanocrystals and nanostructures using simple solution phase techniques. Transmission electron microscopy (TEM) was used as the main tool to study these nanostructures, and also the effect of reaction conditions on their size and morphologies was studied. The materials that were studied were nanocrystalline silicon, germanium and nanostructures of titanium disulphide. Whilst the semiconductors, silicon and germanium were studied because of their applications in biological imaging and as potential fluorescent whitening agents as described in chapter 5; titanium disulphide was studied due to its interesting layered crystal structure and intercalation capabilities.

The thesis is structured in the following way. Chapter 2 discusses the general preparative methods and characterization techniques used to investigate the chemical composition, structure and properties of the materials. In Chapter 3 the synthesis of silicon nanocrystals and there optical properties are described. Chapter 4 discusses the synthesis of germanium nanocrystals by varying reaction parameters, the ease of size control, optical properties and their application in bio-imaging. In chapter 5 the potential application of silicon and germanium nanocrystals as fluorescent whitening agents is described. Chapter 6 details the low temperature synthesis of various morphologies of titanium disulphide by varying reaction parameters. Chapter 7 presents the conclusions and suggests some further work.

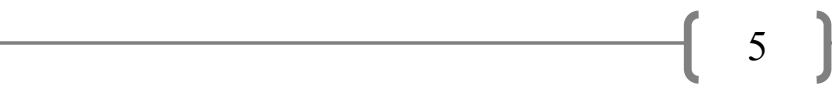




\subsection{Quantum confinement effect}

When one or more dimensions of a particle are brought to the nanoscale regime, the quantum confinement effect sets in. ${ }^{13-15}$ This concept can be explained using the term band gap, a property that is characteristic of semiconductors. ${ }^{16}$ The band gap can be defined as the difference in energy between the valence and conduction band and is typically in the range of $1-3 \mathrm{eV}$ for semiconductors. ${ }^{17-20}$ In bulk materials the energy bands are a continuum of energy levels arising from the overlapping of the atomic orbital's due to the large number of molecules. When a semiconductor is brought to the nanoscale, containing lesser number of molecules, the quantized energy levels become distinct. Thus the addition or removal of atoms significantly changes the energy levels within the band making the band gap size dependent. ${ }^{21-24}$

Energy levels in bulk semiconductor

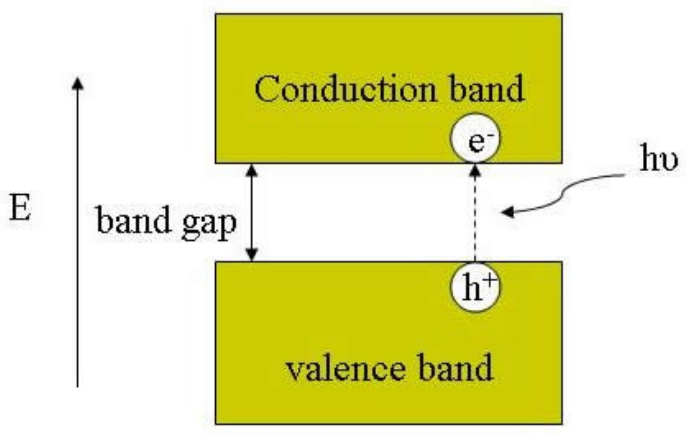

Energy levels in a quantum dot

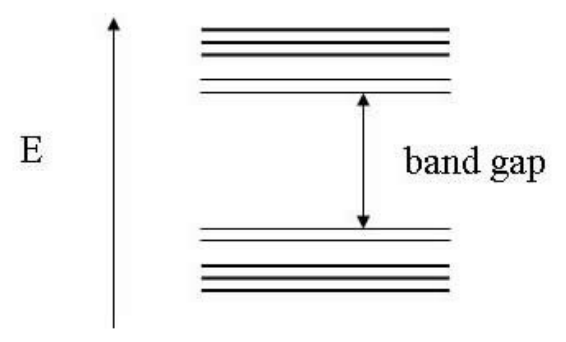

Figure 1.1: A Schematic diagram of the energy levels in a bulk semiconductor and a nanocrystal. 
As a result of this phenomenon the absorption and emission of semiconductor nanocrystals or quantum dots can be customized for different wavelengths. The figure depicts the excitation of an electron into the conduction band thus leaving a hole in the valence band. An electron-hole pair is called an exciton, and the natural physical separation between them is called the excitonic Bohr radius and is characteristic of each material. Thus when a semiconducting material approaches a size nearing its Bohr excitonic radius, the exciton is said to be confined within the particle and is called a quantum $\operatorname{dot}^{25-27}$ 


\subsection{Synthesis and properties of nanocrystals 1.3.1 What are nanocrystals?}

Semiconductor nanocrystals or quantum dots are materials that are typically $2-8 \mathrm{~nm}$ in diameter, consisting of approximately twelve to fourteen thousand atoms. The effect of quantum confinement results, in the electrons and holes in the nanocrystal to exhibit quantized energy states; thus enabling them to exhibit novel physical properties that are not found in their bulk counterparts. Research in semiconductor quantum dots started with the realization that the optical and electronic properties of these particles were strongly dependent on particle size, due to quantum confinement of the charge carriers in small spaces. $1,3,10$

Quantum dots can be prepared through methods ranging from atomic deposition on solid phases to colloidal synthesis in aqueous solutions. Since the size dependent properties of quantum dots are marked when they are monodisperse, a lot of effort is being made to synthesize them in a highly homogeneous and crystalline fashion. In a spherical nanocrystal the free electrons and holes are confined in all three directions and the movement of charge carriers is completely determined by quantum mechanics, hence the name quantum dots. Because of the similarity between the discrete energy levels of quantum dots and the discrete energy levels of atoms (particle in a box) they are often referred to and thought of as "artificial atoms". 6-9 


\subsubsection{Size Dependent properties}

The tailoring of material properties has been shown in many inorganic solids, especially semiconductor nanocrystals. ${ }^{28-30}$ The electronic structure of a nanocrystal depends on it size. ${ }^{13}$ Recent advances have shown that it is an effective route to tune band-gap semiconductor nanocrystals by changing their constituent stoichiometries. ${ }^{31}$ For example in $\mathrm{CdS}$, the following has been observed. The band gap can be tuned between $2.5 \mathrm{eV}$ and 4 $\mathrm{eV}^{1}$ The lowest allowed optical excitation has a radiative rate that ranges from several nanoseconds to tens of picoseconds. ${ }^{1}$ All these fundamental properties have been found in a material of single chemical composition just by reducing the size of the crystal. The large surface area to volume ratio of these low dimension structures is another interesting aspect that brings into account the importance of surface chemistry on the properties of quantum dots. 


\subsection{Colloidal Synthesis of Semiconductor Quantum}

\section{Dots}

Semiconductor quantum dots have been prepared by both physical and chemical methods. But, physical methods such as molecular beam epitaxy (MBE), ${ }^{32-34}$ metal-organic chemical vapor deposition (MOCVD) and pulsed laser ablation have their own disadvantages and limitations. ${ }^{35-40}$ The nanoparticles that are prepared through such techniques are either attached to a substrate or embedded in a matrix. Moreover the possibility of modifying the surface of a single quantum dot is impossible. Expensive instrumentation and high energy consumption further limit these techniques.

In stark contrast, colloidal synthesis offers the advantage of synthesizing nanocrystals of higher yields that could be easily tailored and manipulated. ${ }^{41}$ The surface of the particles can be passivated by functional organic molecules bound or terminated to the surface making them miscible in any liquid. ${ }^{42-44}$ One of the ground breaking advances in the synthesis of colloidal quantum dots is its potential advantage over conventional biological probes ${ }^{45-47}$ Molecules that are capable of emitting light and fluoresce are conjugated with a bio-molecule and used to image the molecules location or probe its environment. ${ }^{48}$ Detailed reviews by Alivisatos on the biological applicability of colloidal quantum dots could be found in the reference section. ${ }^{49,50}$ 


\subsubsection{Nanocrystal nucleation, crystal growth and surface}

\section{stabilization}

Studies have shown that the synthesis of nanocrystals requires a fast and discrete nucleation step followed by a slower controlled growth on the existing nuclei. ${ }^{51}$ The rapid addition of a reagent to a reaction mixture increases the precursor concentration above the nucleation threshold. ${ }^{52}$ A shorter nucleation burst partially eases the supersaturation. As long as the consumption of starting material by the growing nanocrystals does not exceed, the rate of precursor addition, no nuclei would be formed.

Since the growth of an individual nanocrystal is the same as another, the initial size distribution is determined by the time over which the nuclei are formed and begin to grow. If, the growth of nanocrystals during nucleation is small compared with subsequent growth, the nanocrystals tend to become uniform over time and this is referred to as the focusing of size distribution. In short, solution phase synthesis could be said to undergo the following steps. (a) Nucleation, (b) the growth of nuclei into nanocrystals and finally the (c) growth termination and the stabilization of the nanoparticles. ${ }^{53-56}$

Surface stabilizing agents are present in the solution to prevent aggregation and precipitation of the particles. ${ }^{5,54,56}$ When a stabilizing agent is bonded to the surface of a nanocrystal as a monolayer they are called capping agents. ${ }^{25}$ Capping agents help in mediating crystal growth, sterically stabilizing the particles in solution also in passivating the surface electronic states present in the nanocrystal trap states. They also tend to

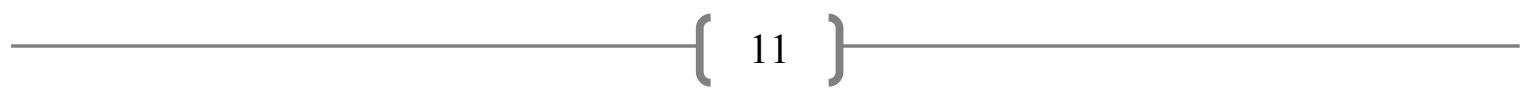


increase the photoluminescence efficiency. ${ }^{47}$ Their ability to protect particles, from oxidation and solubilisation is vital to systems that are susceptible to oxidation. The stability of the nanocrystals, however, with respect to aggregation could be possible only if the capping groups provide a repulsive force that counters the Van der Waal attractions that exist between the nanocrystals. ${ }^{57,58}$

Whilst there are many models to explain the synthesis of colloidal nanocrystals, the LaMer plot is a standard model that explains colloidal growth. The LaMer diagram (figure 1.2) when applied to a system explains that nucleation occurs in the first step of the reaction after which growth of the particle occurs. The size of the particles is expected to increase with concentration of the precursor. This fact is further supported by the reasoning that the number of nuclei is constant and an increase in concentration results in an increase in particle size.

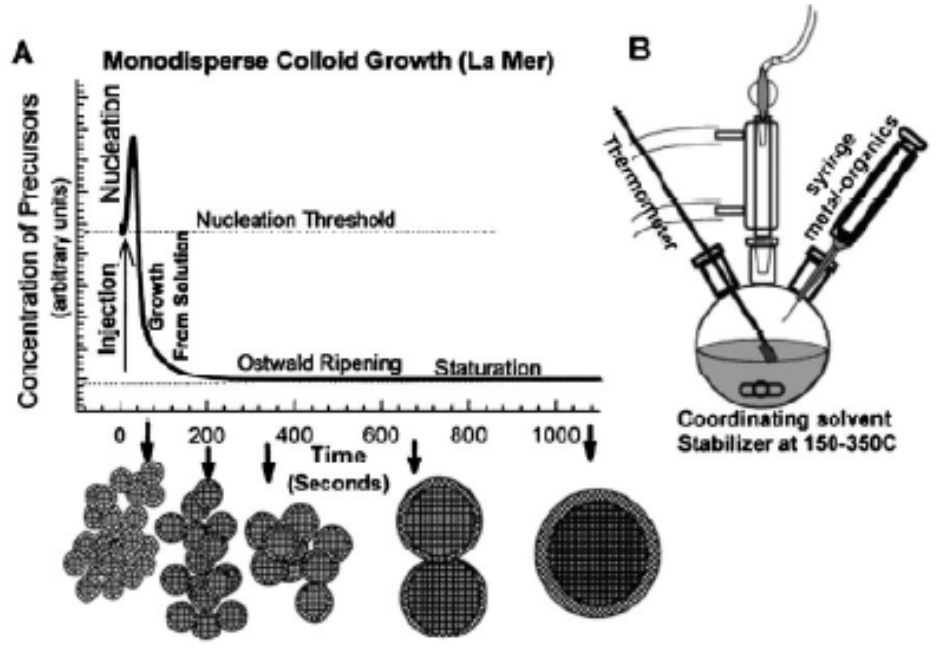

Figure 1.2: The concept of monodisperse colloid growth of La Mer model (A) and a typical synthetic apparatus (B). ${ }^{62 b}$ 


\subsubsection{Semiconductor nanocrystals: from Microemulsion, Solvothermal and Hydrothermal methods}

Microemulsion: Microemulsion routes to synthesize nanoparticles allow one to obtain monodisperse particles and size control by varying the size of the microemulsion droplet radius and the concentration of the precursor. A microemulsion can be defined as a thermodynamically stable, optically transparent dispersion of two immiscible liquids stabilized by a surface active agent. ${ }^{59-61}$ The properties are governed by the watersurfactant molar ratio which is a factor that is linearly correlated with the water droplet size. The mechanism of synthesis of colloidal nanoparticles in microemulsions can be explained by two models. The first is based on the LaMer diagram ${ }^{62 a}$ (explained in 1.4.1) and the second on the thermodynamic stabilization of particles. ${ }^{63}$

The second model suggests that the particles are thermodynamically stabilized by surface active agents and that particle size remains constant though precursor concentration and aqueous droplet size vary. Further nucleation is said to be a continuous process that occurs during the formation of nanoparticles. Both the models are said to be limiting and do not take each other into consideration.

The synthesis of metallic nanoparticles by reduction is by far the most common synthesis in micellar systems. A reverse micellar solution containing a dissolved metal salt and a similar solution containing a reducing agent is added the metal cations are reduced to the metallic state. Such techniques are being increasingly introduced in the synthesis of 
Group IV semiconductors because of the ability to tailor the morphology of the particles and the ease by which their surfaces can be modified. ${ }^{45,64-66}$

Solvothermal and hydrothermal reactions: Solvents can be brought to temperatures well above their boiling points in pressurized vessels such as bombs and autoclaves as a result of the increase in autogenous pressure caused by heating. Reactions performed under such conditions are referred to as solvothermal reactions. ${ }^{67}$ When water is used as a solvent it is termed as a hydrothermal reaction. ${ }^{68}$ When solvents are subjected to conditions above their critical point they are referred to as supercritical solvents. These fluids exhibit properties of both liquid and gas and have high viscosities. They help in solubilizing compounds that would not be soluble under ambient conditions. Solvothermal reactions allow many materials to be prepared at conditions below those normally required for solid state reactions. The primary advantage of such methods is that the products obtained are highly crystalline and do not require post synthetic treatment such as annealing.

Korgel and co workers reported in 2001, the preparation of alkoxy functionalized silicon nanoparticles by the thermal degradation of diphenylsilane in a supercritical solvent mixture of octanol and Hexane. ${ }^{69}$ A very similar synthesis was used to synthesize Germanium nanoparticles by supercritical solvents by the same workers. ${ }^{70,71}$ Though a variety of solvothermal routes to prepare nanocrystalline metal oxides, nitrides and chalcogenides are reported in the literature, very limited reports could be found on Group IV semiconductors. Since these methods offer a choice of solvents and variable reaction temperatures, nanoparticles of different size and morphology could be obtained. 


\subsection{Photoluminescence in Semiconductor Nanocrystals}

Photoluminescence refers to the emission of light by a material through any process other than blackbody radiation. In PL one measures physical and chemical properties of materials by using photons to induce excited electronic states in the material system and analyzing the optical emission as these states relax. Typically, light is directed onto the sample for excitation, and the emitted luminescence is collected by a lens and passed through an optical spectrometer onto a photon detector. The spectral distribution and time dependence of the emission are related to electronic transition probabilities within the sample, and can be used to provide qualitative information about chemical composition, structure, impurities, kinetic process and energy transfer. Sensitivity is one of the strengths of the PL technique, allowing very small quantities or low concentrations of material to be analyzed. ${ }^{112,113}$

In PL, a material gains energy by absorbing photon at some wavelength by promoting an electron from a low to a higher energy level. This may be described as making a transition from the ground state to an excited state of an atom or molecule, or from the valence band to the conduction band of a semiconductor crystal. The system then undergoes a non-radiative internal relaxation involving interaction with crystalline or molecular vibrational and rotational modes, and the excited electron moves to a more stable excited level, such as the bottom of the conduction band or the lowest vibrational molecular state. After a characteristic lifetime in the excited state, electron will return to the ground state. In the luminescent materials some or all of the energy released during this final transition is in the form of light, in which case the relaxation is called radiative. The wavelength of the emitted light is longer than that of the incident light. Optical 
absorption and photoluminescence spectra are commonly used in the characterization of the size of semiconductor nanocrystals.$^{112,113}$ 


\subsection{Silicon}

Silicon is the most ubiquitous material in the electronic world, because of its relative abundance and low cost. In 1990 Canham discovered that porous silicon, exhibited efficient room temperature photoluminescence at visible energies above the bulk silicon band gap of $1.12 \mathrm{eV} .{ }^{72-74}$ Since the discovery of room temperature photoluminescence from porous silicon, a lot of effort has been devoted to the development of silicon nanomaterial based light emission sources. The luminescence of silicon nanostructures have been studied in various systems, from free standing nanocrystals to multilayered structures. ${ }^{75-77}$ Photoluminescing silicon nanostructures have been fabricated by a variety of physical and chemical techniques. Though each technique has its advantages and disadvantages, the solution phase reduction to produce free standing silicon nanocrystals is unique because of the ability to functionalize the surface by both hydrophilic and hydrophobic molecules, making the material suitable for a variety of biological applications.

Silicon is a bio-inert semiconductor that is electronically very stable. In contrast to all IIVI nanoparticle types, Si exhibits a low inherent toxicity. ${ }^{115}$ While most of the II-VI QDs are known to have a direct band-gap transition, bulk $\mathrm{Si}$ is an indirect band-gap semiconductor. In this case the transition from the bottom of the conduction band to the top of the valence band violates conservation of momentum and is electronically forbidden. ${ }^{115}$ The transition does occur, but only with phonon assistance. That means that the absorption and emission of photon require the additional change in lattice vibration mode. This is an indirect process with a low probability, and for this reason, in contrast 
to its extensive use in electronic devices bulk Si has only found limited optical applications. Based on this, it might be anticipated that Si NPs would tend to have a long PL lifetime and low PL efficiencies when compared to direct band-gap semiconductors. ${ }^{115}$ 


\subsubsection{Crystal Structure and Physical Properties}

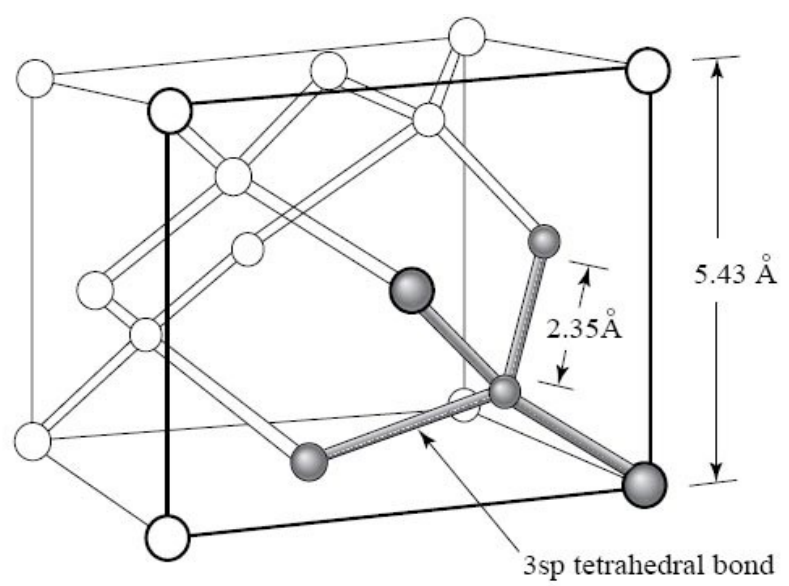

Figure 1.3: The crystal structure of silicon.

Silicon crystallizes in the diamond cubic structure and has two interpenetrating facecentered cubic primitive lattices, as can be seen in figure 1.3. The merged FCC structures are offset by $\mathrm{a} / 4$ in the $\mathrm{x}, \mathrm{y}$ and $\mathrm{z}$ direction. ${ }^{78,79}$

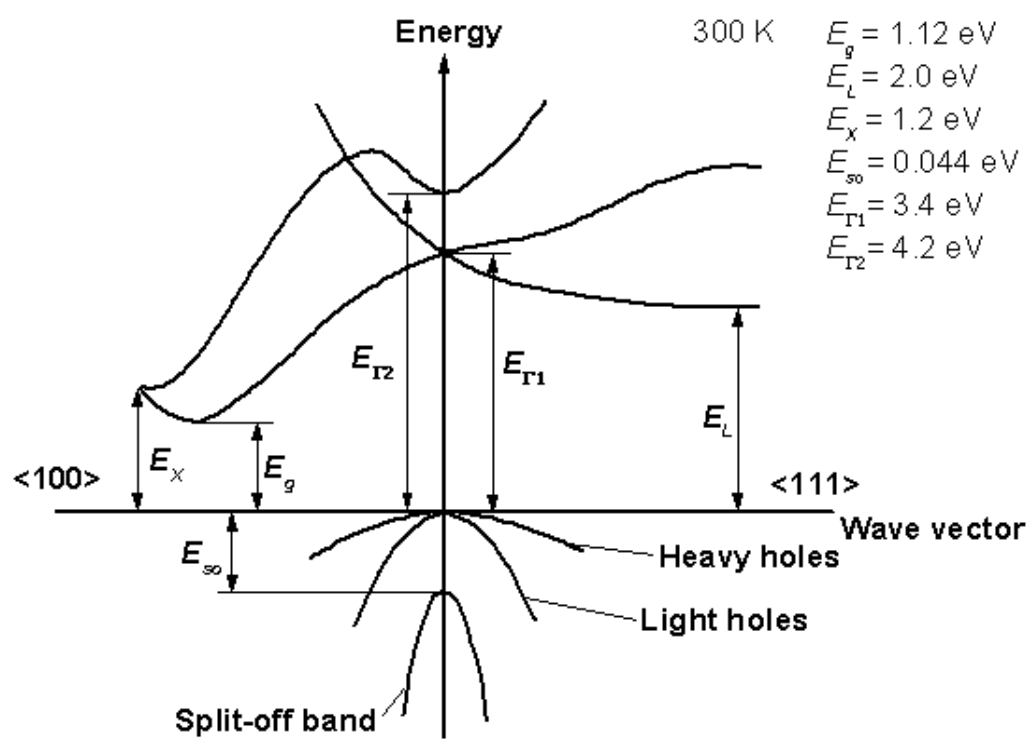

Figure 1.4: The band structure of silicon. 
The band structure of silicon is presented above in figure 1.4. Bulk silicon is an indirect band gap material with an exciton Bohr radius of $\sim 4.9 \mathrm{~nm}$. The basic unit cell parameters and band gap are tabulated below in table $1 .^{80}$

\begin{tabular}{|c|c|}
\hline Structure & Silicon \\
\hline Lattice parameter at 300 K & $0.54311 \mathrm{~nm}$ \\
\hline Band Gap & Indirect \\
\hline Energy of Indirect Band Gap at & $1.12 \mathrm{eV}(1108 \mathrm{~nm})$ \\
$300 \mathrm{~K}$ & $3.4 \mathrm{eV}(366 \mathrm{~nm})$ \\
\hline $\mathrm{K}$ & \\
\hline
\end{tabular}

Table 1: The Basic parameters of silicon 


\subsection{Germanium}

Compared to silicon nanocrystals, germanium particles have a larger exciton Bohr radius, thus imparting a strong quantum confinement independent of the large nanocrystal radius. Though bulk germanium is an indirect band gap material, nanocrystalline germanium has found to behave as direct band gap material. Germanium nanocrystals

exhibiting strong visible photoluminescence have been prepared by several methods. ${ }^{81-84}$ Takeoka and co workers have observed size dependent photoluminescence in the nearinfrared region which is closer to the band gap of bulk germanium $(\sim 0.66 \mathrm{eV}$ at 300 K). ${ }^{85}$ It has also been suggested that the radiative recombination in Ge nanocrystals could be fast because of the small energy difference between the indirect and direct gap of germanium. Due to the narrow band gap $(0.66 \mathrm{eV})$ and large Bohr exciton radius of germanium $(>20 \mathrm{~nm})$ it is possible to tune the photoluminescence of germanium quantum dots throughout the visible spectrum, just by making small changes in the dimension of the particle. These properties make germanium appealing to many optoelectronic applications. ${ }^{86}$ 


\subsubsection{Crystal Structure and Physical Properties}

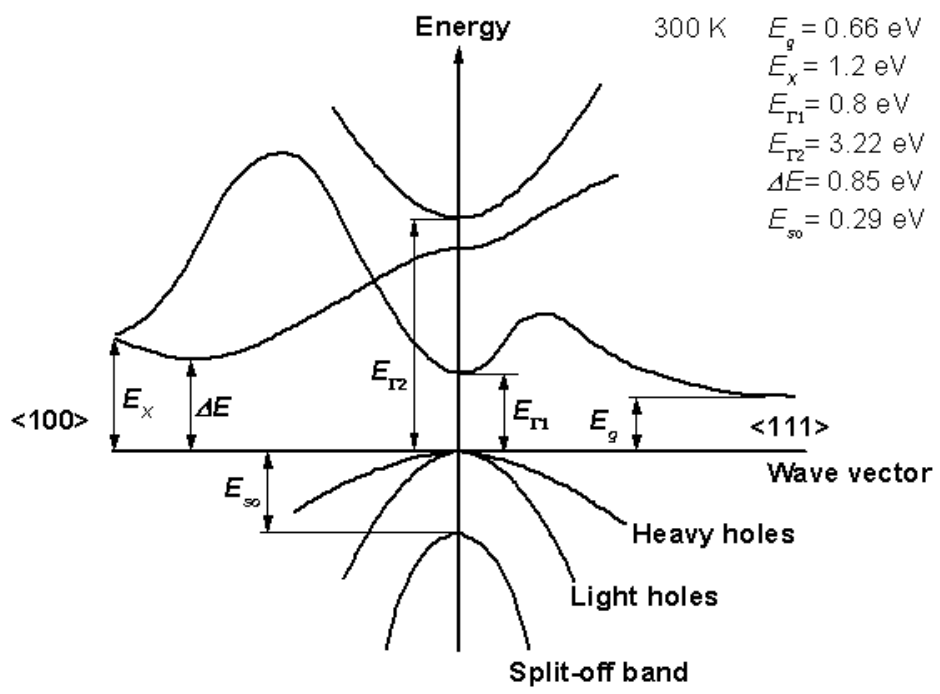

Figure 1.5: The band structure of germanium art $300 \mathrm{~K}$

Germanium like silicon confirms to the diamond crystal structure and varies in that it has a slightly higher lattice parameter. ${ }^{78,79}$ It has a band gap of $0.67 \mathrm{eV}$ which lies in the infrared region, and like silicon if quantum confinement is demonstrated, will widen. The band structure of germanium is presented in figure 1.5. The basic cell parameters and band gap are tabulated below in table $2 .^{80}$

\begin{tabular}{|l|l|}
\hline Function & Germanium \\
\hline Lattice parameter at 300 K & $0.565791 \mathrm{~nm}$ \\
\hline Band Gap & Indirect \\
\hline $\begin{array}{l}\text { Energy of Indirect Band Gap at } \\
300 \mathrm{~K}\end{array}$ & $0.66 \mathrm{eV}(2000 \mathrm{~nm})$ \\
\hline $\begin{array}{l}\text { Energy of Direct Band Gap at } \\
300 \mathrm{~K}\end{array}$ & $0.8 \mathrm{eV}(1650 \mathrm{~nm})$ \\
\hline
\end{tabular}

Table 2: The Basic parameters of germanium 


\subsection{Synthesizing Silicon and Germanium Nanocrystals using a Microemulsion route- Objectives}

In this study, the synthetic methodology of Tilley et al's work on microemulsion routes to Si and Ge nanocrystals, were used as a basis. Briefly, the aim of the set of experiments in chapters 3 and 4 was to improve and extend the existing synthetic aspects of Tilley et al's work, by modifying parameters such as surfactants, precursors, reductants and capping agents, and studying its effect on particle size and properties. The primary objectives and reasons, behind using the microemulsion synthesis for silicon and germanium nanocrystals is listed below, although a more comprehensive review and discussion on the shortcomings of other synthetic methodologies will be discussed in the introduction section of chapters 3 and 4 .

- Few reports on solution techniques to synthesize monodisperse, size controlled $\mathrm{Si}$ and Ge nanocrystals that investigate the role of reactants on the nanocrystal.

- To devise a scheme, whereby nanocrystals of different particle sizes could be synthesized by altering reaction parameters.

- To study the effect of particle size and capping agents on the optical properties of the nanocrystals, and finally

- To produce stable, photoluminescent Si and Ge nanocrystals that are either hydrophobic or hydrophilic. 


\subsubsection{Photoluminescence in Silicon Nanocrystal and}

\section{Germanium Nanocrystals}

There has also been much interest in silicon ( $\mathrm{Si}$ ) and germanium (Ge) nanocrystals, especially in regards to the potential of obtaining useful levels of visible photoluminescence (PL) from these materials produced by a variety Indeed, visible PL has been observed from both $\mathrm{Si}$ and Ge nanocrystals of techniques most of which grow the nanocrystals in a glass $\left(\mathrm{SiO}_{2}\right)$ matrix. ${ }^{116}$ These techniques include sputtering, laser ablation, sol-gel processing, ion implantation followed by high temperature annealing. Many methods have been researched over the last few years for light generation and amplification in silicon and germanium. One of the most promising one is based on silicon nanocrystals with the aim of taking advantage of the reduced dimensionality of the nanocrystalline phase $(1-5 \mathrm{~nm}$ in size) where quantum confinement and surface effects play a crucial role. ${ }^{116}$ It has been found that the silicon nanocrystal bandgap increases with decrease in size and a photoluminescence (PL) external efficiency in excess of $23 \%$ is obtained. ${ }^{116} \mathrm{Si}$ nanocrystals based LED with high efficiency has been obtained by using Si NC active layers and achieving separate injection of electrons and holes. Moreover, optical gain under optical pumping has been already demonstrated in a large variety of experimental conditions. After the initial impulse given by the work of Canham on photoluminescence from porous $\mathrm{Si}$, nanostructured silicon has received extensive attention. This activity is mainly centred on the possibility of getting relevant optoelectronic properties from nanocrystalline $\mathrm{Si}^{116}$ The huge efforts made toward matter manipulation at the nanometer scale have been motivated by the fact that desir- 
able properties can be generated by just changing the system dimension and shape. Investigation of phenomena such as the Stokes shift, the PL emission energy versus nanocrystals size, the doping properties, the radiative lifetimes, the nonlinear optical properties, the quantum-confined Stark effect (QCSE), and so on can give a fundamental contribution to the understanding of how the optical response of such systems can be tuned. A considerable amount of work has been done on excited silicon nanocrystal, but a clear understanding of some aspects is still lacking. The question of surface effects, in particular oxidation, has been addressed in the last few years. ${ }^{116}$ Both theoretical calculations and experimental observations have been applied to investigate the possible active role of the interface on the optoelectronic properties of Si NCs. The optical properties of silicon and germanium nanocrystals synthesized by the microemulsion route will be discussed in chapter 3 and 4 . 


\subsection{Fluorescent Whitening Agents}

Fluorescent or optical whitening agents (FWA) have been widely used for improving the whiteness of textile fabrics and paper. ${ }^{87-92}$ These agents possess the property of absorbing, preferentially, ultra-violet light in the wavelength region of $350 \mathrm{~nm}$ followed by emission of a proportion of the absorbed energy in the form of visible light which is violet, blue or greenish in colour, falling mainly in the range of wavelengths $400-500$ $\mathrm{nm}$ with maximum emission at $430-450 \mathrm{~nm} .^{89,93}$

There are two effects that a fluorescent whitening agent could have on an off-white fabric. The color becomes bluer and the brightness or luminescence increases. Such behavior may be compared with the process of 'bluing' with a dye or pigment, an operation which has been common practice in textile finishing. ${ }^{94}$ The blue dye simply absorbs the yellow light which would otherwise be reflected from the sample, thus decreasing the luminescence, and any yellow coloration is changed to a pale bluish-grey which is less objectionable to the eye. The overall result in this case is that less energy, instead of more, is being radiated in the visible region than would be the case with untreated fabric. $^{90}$

The property of fluorescence is possessed by many common organic dyes such as anthracene, stilbenes, coumarins and pyrazoline. However other properties such as its ability to withstand washing, discoloration and remain attached to the textile fibers is equally important. The drawback of using conventional FWA's is the rapid rate by 
which it undergoes photo-yellowing, especially in woolen fabrics, thus hindering its ability to compete well with cotton and other synthetic fibres. ${ }^{95-97}$ The need for a FWA that undergoes a less rapid rate of photo-yellowing coupled with the ability of silicon and germanium to luminesce and emit in the blue has been the motivating factor in this research.

\subsubsection{The Generation of Singlet Oxygen $\left({ }^{1} \mathrm{O}_{2}\right)$, Superoxide}

\section{radicals in Wool, and the Effect of Water on}

\section{Photoyellowing}

The theory that singlet oxygen is involved in the photoyellowing of wool was first proposed in 1976 by Nicholls and co-workers. ${ }^{114}$ The singlet oxygen mechanism involves absorption of the UV components of sunlight by the aromatic amino acid residues in wool protein, followed by energy transfer through the protein chain to tryptophan residues. Tryptophan residues in the singlet excited state then undergo intersystem crossing to an excited, long-lived triplet state which can react with ground state oxygen to produce singlet oxygen (Scheme 1).

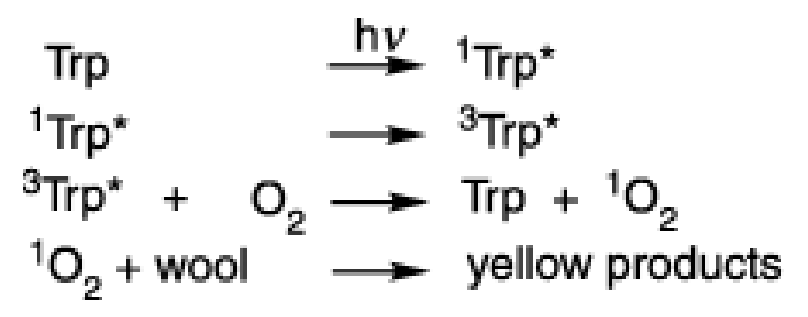

Scheme: 1 (From reference 114) 
Reactive ${ }^{1} \mathrm{O}_{2}$ then reacts with certain amino acid residues in wool, in particular tryptophan, leading to yellow oxidation products ${ }^{114}$. Indirect evidence from the reduced triplet lifetime of ${ }^{3} \mathrm{Trp}^{*}$ in air compared with nitrogen observed in wool phosphorescence studies supports this mechanism ${ }^{114}$. One of the stronger arguments for the singlet oxygen mechanism has been based on measurements of the $\mathrm{O}_{2}$ consumption of wool during irradiation. At least 10 oxygen molecules are consumed per molecule of tryptophan destroyed. ${ }^{114}$ However, it has not been demonstrated that this $\mathrm{O}_{2}$ is reacting with ${ }^{3} \operatorname{Trp}$ to form ${ }^{1} \mathrm{O}_{2}$. Direct oxidation of other excited species in wool is likely to occur concurrently, and many other mechanisms, including the propagation stages of freeradical oxidation, are known to consume large amounts of oxygen.

Millington and Kirschenbaum detected the formation of hydroxyl radicals when wool fabric in the wet state was irradiated with both UV-A $(366 \mathrm{~nm})$ and blue light $(425 \mathrm{~nm})$ in the presence of a highly specific and sensitive fluorescent probe, the terephthalate anion. ${ }^{114}$ Trace metal ions (especially $\mathrm{Fe}$ and $\mathrm{Cu}$ in wool) are involved in ${ }^{\circ} \mathrm{OH}$ generation since $\left[{ }^{\circ} \mathrm{OH}\right]$ was significantly reduced in the presence of the metal chelator deferoxamine. Similar behaviour was observed for nylon, cotton and polyester fabrics, although wool produced the highest [ $\left.{ }^{\circ} \mathrm{OH}\right]$.

Millington and Kirschenbaum. ${ }^{114}$ suggested that the photoyellowing of wool in the wet state occurs via the formation of hydroxyl and superoxide radicals catalysed by trace metal ions that are strongly bound to wool. This is the photodegradation mechanism that is known to occur in synthetic polymers. It involves the formation of free radicals as a consequence of the excitation of absorbing chromophores, particularly in the UV region. 
114 The presence of trace amounts of certain metal ions has been shown to significantly increase the production of free radicals in irradiated polymers. . ${ }^{114}$ The most active metal ions are those which are readily oxidised or reduced by one electron transfer and have different oxidation states with similar stability, and in particular the transition metals $\mathrm{Fe}, \mathrm{Cu}, \mathrm{Mn}, \mathrm{Co}$ and V. This has been demonstrated for wool, where doping the fabric with $\mathrm{Fe}^{\mathrm{III}}$ and $\mathrm{Cu}^{\mathrm{II}}$ ions increased the production of free radicals after irradiation at wavelengths above $320 \mathrm{~nm}$ in ESR studies. ${ }^{114}$ Copper(ii) binds to wool and this complex has been shown to enhance hydroxyl radical production in the presence of hydrogen peroxide. ${ }^{114}$ A previous study has shown that human hair ${ }^{114}$, which is very similar in structure and composition to wool, contains significant amounts of trace metals that appear to be strongly bound. Wool also contains trace metals, in particular iron and copper. Analysis of hydrolysed Merino wool fabric using inductively coupled plasma atomic emission spectroscopy (ICP-AES) has reported $c a .15 \mathrm{ppm} \mathrm{Fe,} c a .5 \mathrm{ppm} \mathrm{Cu}$ and ca. 2 ppm Mn . ${ }^{114}$

Once free-radical species are formed via photochemical initiation, these react rapidly with molecular oxygen and initiate the typical polymer photodegradation chain reaction shown in scheme 2. ${ }^{114}$ During the propagation stages, significant levels of polymer hydro peroxides $(\mathrm{POOH})$ are produced. In the presence of transition metal ions or light, hydro peroxides can decompose to produce aggressive hydroxyl radicals (Eqn 10). 


$$
\begin{aligned}
& \text { Initiation } \\
& \mathrm{I} \stackrel{\mathrm{h} v}{\longrightarrow} \mathrm{I}^{*} \\
& \mathrm{I}^{*} \longrightarrow \mathrm{A}^{*}+\mathrm{B}^{*} \\
& \mathrm{~A}^{*}+\mathrm{PH} \longrightarrow \mathrm{P}^{*}+\mathrm{AH} \\
& \mathrm{POO}^{*}+\mathrm{PH} \longrightarrow \mathrm{POOH}+\mathrm{P}^{*} \\
& \text { Branching } \quad \mathrm{POOH} \stackrel{\mathrm{h} v \text { or } \mathrm{M}^{n+}}{\longrightarrow} \mathrm{PO} \cdot+\cdot{ }^{\cdot} \mathrm{OH} \\
& \mathrm{PO}^{*}+\mathrm{PH} \longrightarrow \mathrm{POH}+\mathrm{P}^{*} \\
& \cdot \mathrm{OH}+\mathrm{PH} \longrightarrow \mathrm{H}_{2} \mathrm{O}+\mathrm{P}^{\cdot} \\
& \mathrm{POOH}+\mathrm{PH} \stackrel{\text { hvor } \mathrm{M}^{n+}}{\longrightarrow} \mathrm{PO}^{*}+\mathrm{P}^{*}+\mathrm{H}_{2} \mathrm{O} \\
& 2 \mathrm{POOH} \stackrel{\mathrm{h} v \text { or } \mathrm{M}^{n+}}{\longrightarrow} \mathrm{POO}^{*}+\mathrm{PO}^{*}+\mathrm{H}_{2} \mathrm{O} \\
& \mathrm{P}^{*}+\mathrm{P}^{*} \longrightarrow \mathrm{P}-\mathrm{P} \\
& \mathrm{P}^{*}+\mathrm{POO}^{*} \longrightarrow \text { POOP } \\
& \mathrm{POO}^{*}+\mathrm{POO}^{*} \longrightarrow \mathrm{POOP}+\mathrm{O}_{2} \\
& \mathrm{PH}=\text { polymer } \\
& \text { I = primary photochemical absorber (radical initiator) } \\
& \mathrm{A}^{\boldsymbol{*}} \text { and } \mathrm{B}^{\boldsymbol{*}}=\text { radical products from excited state of initiator } \\
& \mathrm{M}^{n+}=\text { catalytic metal ions }
\end{aligned}
$$

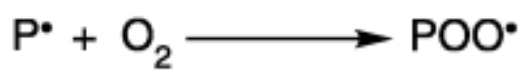

Scheme 2: (From Reference 114) 
Another suggested route to hydroxyl radicals is via the superoxide radical anion. Potential mechanisms to generate this radical and its subsequent reactions are shown in Scheme 3. This reaction scheme has been shown to be very important in many biological systems ${ }^{114}$, recently including melanin and human hair. Tryptophan (via its oxidation products) has also been shown to generate superoxide and hydrogen peroxide directly when exposed to near-UV radiation in the range of $300-375 \mathrm{~nm}$, which is particularly relevant for wool and silk ${ }^{114}$. The superoxide anion undergoes dismutation to produce hydrogen peroxide that can then form hydroxyl radicals through the Fenton reaction in the presence of trace metal ions and through direct photolysis of hydrogen peroxide (Eqns 21 and 22).

$$
\begin{aligned}
& \mathrm{Chr}+\mathrm{hv} \longrightarrow \mathrm{Chr}^{*} \\
& \mathrm{Chr}^{*}+\mathrm{O}_{2} \longrightarrow \mathrm{Chr}^{+\bullet}+\mathrm{O}_{2} \cdot \\
& 2 \mathrm{O}_{2}^{-\bullet}+2 \mathrm{H}^{+} \longrightarrow \mathrm{H}_{2} \mathrm{O}_{2}+\mathrm{O}_{2} \\
& \mathrm{H}_{2} \mathrm{O}_{2}+\mathrm{Mn}^{+} \longrightarrow \mathrm{M}^{(n+1)+}+\mathrm{OH}^{*}+\mathrm{H}^{-} \\
& \mathrm{H}_{2} \mathrm{O}_{2}+\mathrm{hv} \longrightarrow 2 \mathrm{H}^{*} \\
& \mathrm{M}^{(n+1)+}+\mathrm{O}_{2} \longrightarrow \mathrm{Mn}^{+}+\mathrm{O}_{2} \\
& \mathrm{Chr}^{(n+}=\text { polymer chromophore or impurity } \\
& \mathrm{Mn}^{+}, \mathrm{M}^{(n+1)+}=\text { catalytic metal ions }
\end{aligned}
$$

Scheme 3: (From reference 114)

The photoyellowing of proteinaceous fibers, particularly in the wet state, might occur via free radical mechanisms in a similar manner to other polymer systems exposed to sunlight. ESR spectroscopy has shown that both carbon- and sulphur-centred free radicals are formed directly in wool after irradiation in the absence of atmospheric oxygen. In the presence of air or oxygen, these signals are to some extent quenched, 
indicating that the radicals are reacting with oxygen. One would expect the catalytic influence of trace metal ions, and in particular the generation of hydroxyl radicals via the Fenton reaction, to be much greater under wet irradiation conditions than dry. This is the case for other systems where redox mechanisms are involved, such as the corrosion of metals. ${ }^{114}$ 


\subsection{Titanium Disulphide}

Metal di-chalcogenide materials with layered structures such as titanium disulphide have attracted considerable interest in recent years because of their electronic properties and potential applications. ${ }^{98-100}$ Titanium disulphide and its intercalation compounds have been studied intensively and shown to be promising cathode materials in rechargeable batteries and as potential hydrogen storage devices. ${ }^{101,102}$

\subsubsection{Crystal Structure and Physical Properties}

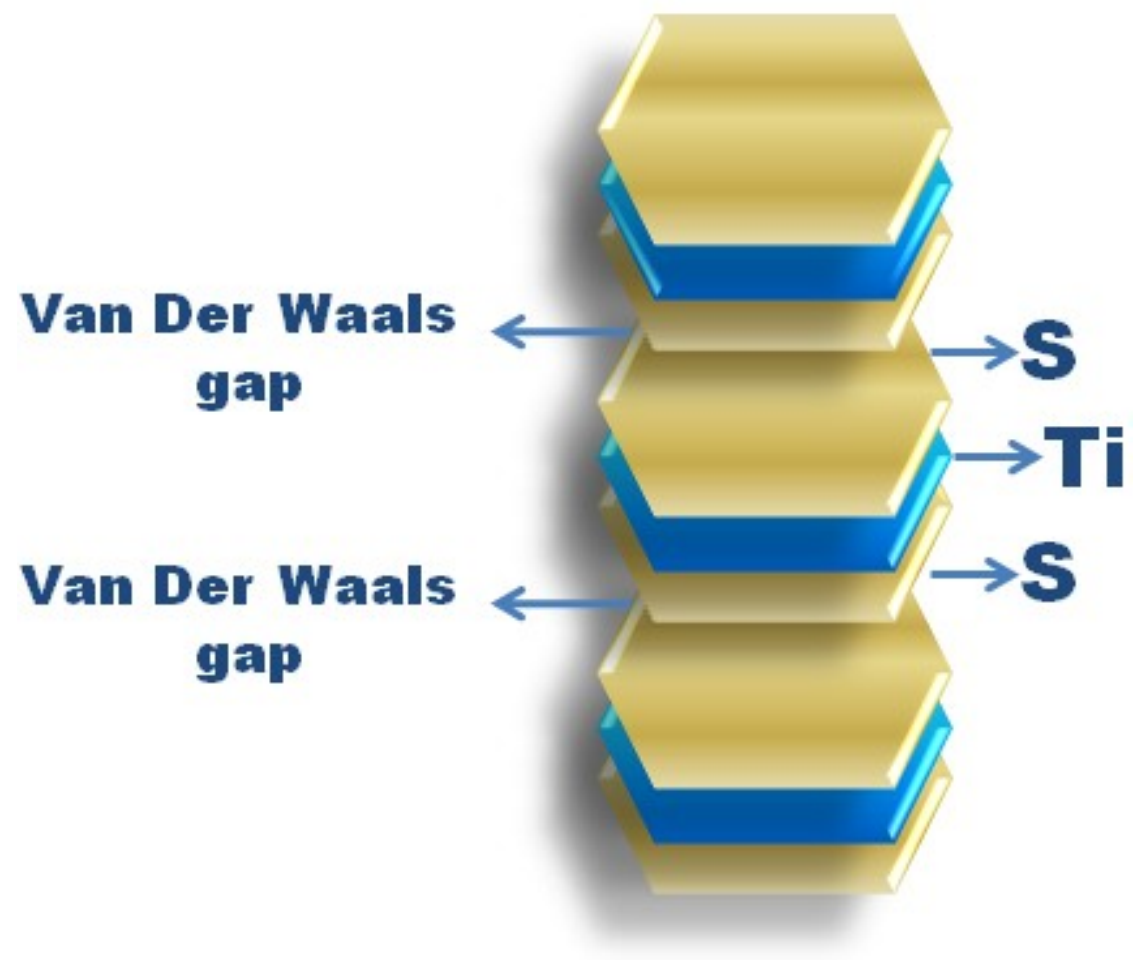

Figure 1.6: A pictorial representation of the hexagonal layered structure of $\mathrm{TiS}_{2}$ 
Titanium disulphide like the rest of the group IVA dichalcogenides crystallizes in the hexagonal $\mathrm{CdI}_{2}$ structure, a layered compound with repeating subunits, made by a layer of $\mathrm{Ti}$ atoms and a layer of $\mathrm{S}$ atoms. Figure 1.6 demonstrates the sequential layered crystal structure of titanium disulphide. There is strong bonding between atoms in the $\mathrm{Ti}$ and S layers, whereas weak van der Waals forces dominate the bonding between the S and S layers. ${ }^{103}$ These weak forces of attraction are the reason for the large stability of the [001] surface of the layers, allowing the material to accommodate extra atoms in the van der Waals region. The sulphur atoms are octahedrally coordinated to the titanium atoms, with each $\mathrm{TiS}_{6}$ octahedra combined with each other tightly through strong covalent bonds, while each layer stacks weakly by van der Waals force as can be seen in figure 1.7. ${ }^{104}$
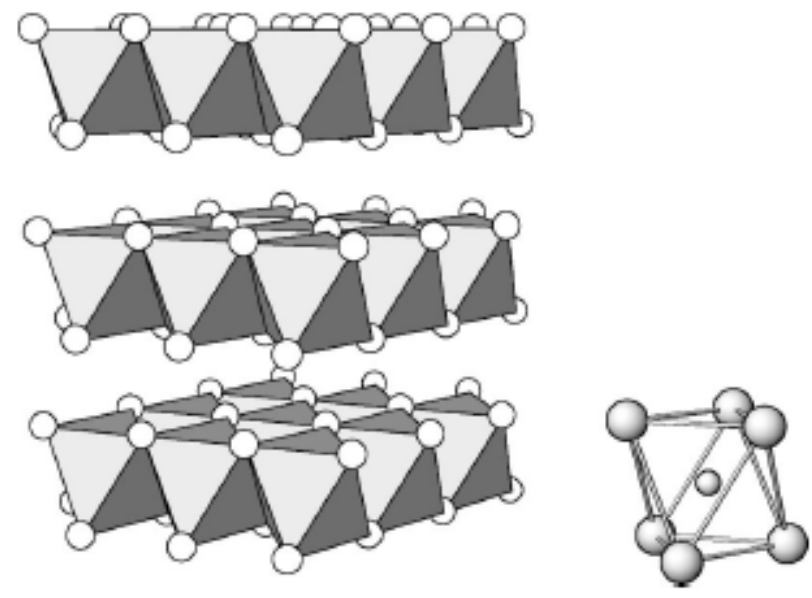

Figure 1.7: The crystal structure of $\mathrm{TiS}_{2}$ and an individual octahedral unit ${ }^{109}$

Bulk titanium disulphide is known to be a semiconductor with a band gap of about $2 \mathrm{eV}$ and an indirect bandgap of $1.4 \mathrm{eV}$. The Bohr radius of titanium disulphide has been estimated to be $6.43 \mathrm{~nm}$. The basic unit cell parameters, band gaps and exciton bohr radius of titanium disulphide is shown in table $3 .{ }^{104}$ 


\begin{tabular}{|c|c|}
\hline Property & Titanium Disulphide \\
\hline Lattice Parameter at $300 \mathrm{~K}$ & $\mathrm{a}=3.40 \AA$ \\
$\mathrm{c}=5.69 \AA$
\end{tabular}

Table 3: The basic parameters of $\mathrm{TiS}_{2}$

\subsubsection{Titanium disulphide Nanostructures}

Various morphologies of $\mathrm{TiS}_{2}$ such as nanotubes, ${ }^{101}$ nanoclusters, ${ }^{102}$ fullerenes, ${ }^{105}$ nanodisks, ${ }^{106}$ whiskers, ${ }^{107}$ and thin films ${ }^{108}$ have been successfully synthesized. With respect to the synthesis of three-dimensional (3D) nanostructures, morphologies such as flower-like patterns and dendritic crystals of $\mathrm{TiS}_{2}$ have been synthesized, by a chemical vapor transport approach ${ }^{109}$ However, the controlled wet chemical synthesis of $3 \mathrm{D}$ nanostructures of $\mathrm{TiS}_{2}$ has not previously been achieved and has proved to be a motivational factor in this study. Reports have been observed for the synthesis of flake-like nanostructures for materials such as $\mathrm{SnS}_{2}$ and $\mathrm{MoS}_{2}$, their growth attributed to their crystal structure. ${ }^{110,111}$ The synthesis of flake-like 2D nanostructures has also not been studied or its synthesis reported in the literature. Moreover, the facile control of whether materials adopt two-dimensional (2D) structures such as nanoflakes or nanoplatelets and $3 \mathrm{D}$ structures such as nanoflowers or nanorods is still difficult and requires complex synthetic techniques. ${ }^{108,109}$ However, a colloidal synthesis offers a more promising approach with advantages of being low cost in preparation and suitable for large-scale 
production. Chapter 6 of this thesis will be mainly concerned with the colloidal synthesis of various $\mathrm{TiS}_{2}$ nanostructures. 


\subsection{References}

1. Alivisatos, A. P., Semiconductor clusters, nanocrystals, and quantum dots. Science 1996, 271 (5251), 933-937.

2. Alivisatos, A. P., Nanocrystals: Building blocks for modern materials design. Endeavour 1997, 21 (2), 56-60.

3. Whitesides, G. M., Nanoscience, nanotechnology, and chemistry. Small 2005, 1 (2), 172-179.

4. Alivisatos, A. P.; Barbara, P. F.; Castleman, A. W.; Chang, J.; Dixon, D. A.; Klein, M. L.; McLendon, G. L.; Miller, J. S.; Ratner, M. A.; Rossky, P. J.; Stupp, S. I.; Thompson, M. E., From molecules to materials: Current trends and future directions. Advanced Materials 1998, 10 (16), 1297-1336.

5. Ozin, G. A., Nanochemistry : a chemical approach to nanomaterials. Cambridge : Royal Society of Chemistry: 2009.

6. Puntes, V. F.; Krishnan, K. M.; Alivisatos, A. P., Colloidal nanocrystal shape and size control: The case of cobalt. Science 2001, 291 (5511), 2115-2117.

7. Puntes, V. F.; Krishnan, K. M.; Alivisatos, P., Synthesis, self-assembly, and magnetic behavior of a two-dimensional superlattice of single-crystal epsilon-Co nanoparticles. Applied Physics Letters 2001, 78 (15), 2187-2189. 
8. Jana, N. R.; Gearheart, L.; Murphy, C. J., Wet chemical synthesis of silver nanorods and nanowires of controllable aspect ratio. Chemical Communications 2001, (7), 617-618.

9. Jana, N. R.; Gearheart, L.; Murphy, C. J., Wet chemical synthesis of high aspect ratio cylindrical gold nanorods. Journal of Physical Chemistry B 2001, 105 (19), 4065-4067.

10. Alivisatos, A. P., Perspectives on the physical chemistry of semiconductor nanocrystals. Journal of Physical Chemistry 1996, 100 (31), 13226-13239.

11. Parak, W. J.; Gerion, D.; Pellegrino, T.; Zanchet, D.; Micheel, C.; Williams, S. C.; Boudreau, R.; Le Gros, M. A.; Larabell, C. A.; Alivisatos, A. P., Biological applications of colloidal nanocrystals. Nanotechnology 2003, 14 (7), R15-R27.

12. Yin, Y.; Alivisatos, A. P., Colloidal nanocrystal synthesis and the organicinorganic interface. Nature 2005, 437 (7059), 664-670.

13. Rao, C. N. R.; Kulkarni, G. U.; Thomas, P. J.; Edwards, P. P., Sizedependent chemistry: Properties of nanocrystals. Chemistry-a European Journal 2002, $8(1), 29-35$.

14. Artemyev, M. V.; Bibik, A. I.; Gurinovich, L. I.; Gaponenko, S. V.; Woggon, U., Evolution from individual to collective electron states in a dense quantum dot ensemble. Physical Review B 1999, 60 (3), 1504-1506. 
15. Busani, R.; Folkers, M.; Cheshnovsky, O., Direct observation of band-gap closure in mercury clusters. Physical Review Letters 1998, 81 (18), 3836-3839.

16. Haug, H. K., S. W., Quantum Theory of the Optical and Electronic Properties of Semiconductors. 1994.

17. Klingshirn, C., Semiconductor Optics Springer; 2nd edition: 2005.

18. Schäfer, W., Wegener, Martin, Semiconductor Optics and Transport Phenomena. Springer: 2002.

19. Zimmermann, R., Many-Particle Theory of Highly Excited Semiconductors. 1988.

20. Sapra, S.; Sarma, D. D., Evolution of the electronic structure with size in II-VI semiconductor nanocrystals. Physical Review B 2004, 69 (12), -.

21. Halperin, W. P., Quantum Size Effects in Metal Particles. Reviews of Modern Physics 1986, 58 (3), 533-606.

22. Henglein, A., Electronics of Colloidal Nanometer Particles. Berichte Der Bunsen-Gesellschaft-Physical Chemistry Chemical Physics 1995, 99 (7), 903913.

23. Jortner, J., Cluster Size Effects. Zeitschrift Fur Physik D-Atoms Molecules and Clusters 1992, 24 (3), 247-275. 
24. Link, S.; El-Sayed, M. A., Size and temperature dependence of the plasmon absorption of colloidal gold nanoparticles. Journal of Physical Chemistry B 1999, 103 (21), 4212-4217.

25. Buhro, W. E.; Colvin, V. L., Semiconductor nanocrystals - Shape matters. Nature Materials 2003, 2 (3), 138-139.

26. Koch, S. W.; Kira, M.; Khitrova, G.; Gibbs, H. M., Semiconductor excitons in new light. Nature Materials 2006, 5 (7), 523-531.

27. Klingshirn, C.; Haug, H., Optical-Properties of Highly Excited Direct Gap Semiconductors. Physics Reports-Review Section of Physics Letters 1981, 70 (5), 315-398.

28. Kan, S.; Mokari, T.; Rothenberg, E.; Banin, U., Synthesis and size-dependent properties of zinc-blende semiconductor quantum rods (vol 2, pg 155, 2003). $\mathrm{Na}$ ture Materials 2004, 3 (1), 72-72.

29. Banin, U.; Cao, Y. W.; Katz, D.; Millo, O., Identification of atomic-like electronic states in indium arsenide nanocrystal quantum dots. Nature 1999, 400 (6744), 542-544.

30. Liu, L. P.; Zhuang, Z. B.; Xie, T.; Wang, Y. G.; Li, J.; Peng, Q.; Li, Y. D., Shape Control of CdSe Nanocrystals with Zinc Blende Structure. Journal of the American Chemical Society 2009, 131 (45), 16423-16429. 
31. Zhong, X. H.; Feng, Y. Y., New strategy for band-gap tuning in semiconductor nanocrystals. Research on Chemical Intermediates 2008, 34 (2-3), 287-298.

32. Feit, Z.; Kostyk, D.; Woods, R. J.; Mak, P., Single-Mode Molecular-Beam Epitaxy Grown Pbeusete/Pbte Buried-Heterostructure Diode-Lasers for Co2 High-Resolution Spectroscopy. Applied Physics Letters 1991, 58 (4), 343-345.

33. Springhotz, G.; Bauer, G., Molecular beam epitaxy of IV-VI semiconductor hetero- and nano-structures. Physica Status Solidi B-Basic Solid State Physics 2007, 244 (8), 2752-2767.

34. Dekel, E.; Gershoni, D.; Ehrenfreund, E.; Spektor, D.; Garcia, J. M.; Petroff, P. M., Multiexciton spectroscopy of a single self-assembled quantum dot. Physical Review Letters 1998, 80 (22), 4991-4994.

35. Shalini, K.; Chandrasekaran, S.; Shivashankar, S. A., Growth of nanocrystalline TiO2 films by MOCVD using a novel precursor. Journal of Crystal Growth 2005, 284 (3-4), 388-395.

36. Domrachev, G. A.; Ob'edkov, A. M.; Kaverin, B. S.; Zaitsev, A. A.; Titova, S. N.; Kirillov, A. I.; Strahkov, A. S.; Ketkov, S. Y.; Domracheva, E. G.; Zhogova, K. B.; Kruglova, M. V.; Filatov, D. O.; Bukalov, S. S.; Mikhalitsyn, L. A.; Leites, L. A., MOCVD synthesis of germanium filled "diamondlike" carbon nanotubes and microtubes from organogermanium precursors and their field-emission properties. Chemical Vapor Deposition 2006, 12 (6), 357-363. 
37. Kaganovich, E. B.; Manoilov, E. G.; Begun, E. V., Photoluminescence of germanium quantum dots formed by pulsed laser ablation. Semiconductors 2007, $41(2), 172-175$.

38. Riabinina, D.; Durand, C.; Chaker, M.; Rowell, N.; Rosei, F., A novel approach to the synthesis of photoluminescent germanium nanoparticles by reactive laser ablation. Nanotechnology 2006, 17 (9), 2152-2155.

39. Semaltianos, N. G.; Logothetidis, S.; Perrie, W.; Romani, S.; Potter, R. J.; Edwardson, S. P.; French, P.; Sharp, M.; Dearden, G.; Watkins, K. G., Silicon nanoparticles generated by femtosecond laser ablation in a liquid environment. Journal of Nanoparticle Research 2010, 12 (2), 573-580.

40. Nath, M.; Rao, C. N. R.; Popovitz-Biro, R.; Albu-Yaron, A.; Tenne, R., Nanoparticles produced by laser ablation of HfS3 in liquid medium: Inorganic fullerene-like structures of Hf2S. Chemistry of Materials 2004, 16 (11), 22382243.

41. Lee, D. C.; Pietryga, J. M.; Robel, I.; Werder, D. J.; Schaller, R. D.; Klimov, V. I., Colloidal Synthesis of Infrared-Emitting Germanium Nanocrystals. Journal of the American Chemical Society 2009, 131 (10), 3436-+.

42. Shiohara, A.; Hanada, S.; Prabakar, S.; Fujioka, K.; Lim, T. H.; Yamamoto, K.; Northcote, P. T.; Tilley, R. D., Chemical Reactions on Surface Molecules Attached to Silicon Quantum Dots. Journal of the American Chemical Society 2010, 132 (1), 248-253. 
43. Prabakar, S.; Shiohara, A.; Hanada, S.; Fujioka, K.; Yamamoto, K.; Tilley, R. D., Size Controlled Synthesis of Germanium Nanocrystals by Hydride Reducing Agents and Their Biological Applications. Chemistry of Materials 2010, 22 (2), 482-486.

44. Fujioka, K.; Hiruoka, M.; Sato, K.; Manabe, N.; Miyasaka, R.; Hanada, S.; Hoshino, A.; Tilley, R. D.; Manome, Y.; Hirakuri, K.; Yamamoto, K., Luminescent passive-oxidized silicon quantum dots as biological staining labels and their cytotoxicity effects at high concentration. Nanotechnology 2008, 19 (41), -

45. Tilley, R. D.; Warner, J. H.; Yamamoto, K.; Matsui, I.; Fujimori, H., Microemulsion synthesis of monodisperse surface stabilized silicon nanocrystals. Chemical Communications 2005, (14), 1833-1835.

46. Warner, J. H.; Hoshino, A.; Yamamoto, K.; Tilley, R. D., Water-soluble photoluminescent silicon quantum dots. Angewandte Chemie-International Edition 2005, 44 (29), 4550-4554.

47. Warner, J. H.; Rubinsztein-Dunlop, H.; Tilley, R. D., Surface morphology dependent photoluminescence from colloidal silicon nanocrystals. Journal of Physical Chemistry B 2005, 109 (41), 19064-19067.

48. Fujioka, K.; Hoshino, A.; Manabe, N.; Futamura, Y.; Tilley, R.; Yamamoto, K., Silicon nanocrystals as handy biomarkers - art. no. 644816. Colloidal Quantum Dots for Biomedical Applications II 2007, 6448, 44816-44816 
49. Alivisatos, P., The use of nanocrystals in biological detection. Nature Biotechnology 2004, 22 (1), 47-52.

50. Bruchez, M.; Moronne, M.; Gin, P.; Weiss, S.; Alivisatos, A. P., Semiconductor nanocrystals as fluorescent biological labels. Science 1998, 281 (5385), 20132016.

51. Everett, D. H., Basic Principles of Colloid Science. The Royal Society of Chemistry London: 1988.

52. Burda, C.; Chen, X. B.; Narayanan, R.; El-Sayed, M. A., Chemistry and properties of nanocrystals of different shapes. Chemical Reviews 2005, 105 (4), 1025-1102.

53. Peng, X. G.; Wickham, J.; Alivisatos, A. P., Kinetics of II-VI and III-V colloidal semiconductor nanocrystal growth: "Focusing" of size distributions. Journal of the American Chemical Society 1998, 120 (21), 5343-5344.

54. Murray, C. B.; Kagan, C. R.; Bawendi, M. G., Synthesis and characterization of monodisperse nanocrystals and close-packed nanocrystal assemblies. Annual Review of Materials Science 2000, 30, 545-610.

55. Pamplin, B. R., Crystal Growth. Pergamon Press: New York: 1975.

56. Murray, C. B.; Norris, D. J.; Bawendi, M. G., Synthesis and Characterization of Nearly Monodisperse Cde $(\mathrm{E}=\mathrm{S}$, Se, Te) Semiconductor Nanocrystallites. Journal of the American Chemical Society 1993, 115 (19), 8706-8715. 
57. McBann, J. W.; Salmon, C. S., Colloidal electrolytes. Soap solutions and their constitution. Journal of the American Chemical Society 1920, 42, 426-460.

58. Gutmann, H.; Kertes, A. S., Cationic Surfactants in Organic-Solvents .3. Critical Micelle Concentration of Dodecylammonium Halides in Benzene and Toluene. Journal of Colloid and Interface Science 1975, 51 (3), 406-411.

59. Garcia-Rio, L.; Leis, J. R.; Moreira, J. A., Reactivity in water/oil microemulsions. Influence of sodium bis(2-ethylhexyl)sulfosuccinate/isooctane/water microemulsions on the solvolysis mechanism of substituted benzoyl chlorides. Journal of the American Chemical Society 2000, 122 (42), 10325-10334.

60. Garcia-Rio, L.; Leis, J. R.; Mejuto, J. C., Solvolysis of benzoyl halides in AOT/isooctane/water microemulsions. Influence of the leaving group. Langmuir 2003, $19(8), 3190-3197$.

61. Garcia-Rio, L.; Leis, J. R., Reactivity in quaternary water in oil microemulsions. 2. Different distribution of the reagents changing from three- to fourcomponent microemulsions. Journal of Physical Chemistry B 2000, 104 (28), 6618-6625.

62.(a) Lamer, V. K.; Dinegar, R. H., Theory, Production and Mechanism of Formation of Monodispersed Hydrosols. Journal of the American Chemical Society 1950, 72 (11), 4847-4854; (b) Blackman, J. A., Metallic Nanoparticles, Elsevier, 2009. 
63. Leff, D. V.; Ohara, P. C.; Heath, J. R.; Gelbart, W. M., Thermodynamic Control of Gold Nanocrystal Size - Experiment and Theory. Journal of Physical Chemistry 1995, 99 (18), 7036-7041.

64. Ma, X. C.; Wu, F. Y.; Kauzlarich, S. M., Alkyl-terminated crystalline Ge nanoparticles prepared from $\mathrm{NaGe}$ Synthesis, functionalization and optical properties. Journal of Solid State Chemistry 2008, 181 (7), 1628-1633.

65. Zhang, X. M.; Neiner, D.; Wang, S. Z.; Louie, A. Y.; Kauzlarich, S. M., A new solution route to hydrogen-terminated silicon nanoparticles: synthesis, functionalization and water stability. Nanotechnology 2007, 18 (9), -.

66. Chiu, H. W.; Kauzlarich, S. M.; Sutter, E., Solution synthesis and characterization of Ge nanoparticles prepared via reduction. Abstracts of Papers of the American Chemical Society 2004, 227, U1308-U1308.

67. Choucair, M.; Thordarson, P.; Stride, J. A., Gram-scale production of graphene based on solvothermal synthesis and sonication. Nature Nanotechnology 2009, 4 (1), 30-33.

68. Wang, X.; Li, Y. D., Selected-control hydrothermal synthesis of alpha- and beta-MnO2 single crystal nanowires. Journal of the American Chemical Society 2002, 124 (12), 2880-2881.

69. Holmes, J. D.; Ziegler, K. J.; Doty, R. C.; Pell, L. E.; Johnston, K. P.; Korgel, B. A., Highly luminescent silicon nanocrystals with discrete optical 
transitions. Journal of the American Chemical Society 2001, 123 (16), 37433748.

70. Lu, X. M.; Ziegler, K. J.; Ghezelbash, A.; Johnston, K. P.; Korgel, B. A., Synthesis of germanium nanocrystals in high temperature supercritical fluid solvents. Nano Letters 2004, 4 (5), 969-974.

71. Hanrath, T.; Korgel, B. A., Nucleation and growth of germanium nanowires seeded by organic monolayer-coated gold nanocrystals. Journal of the American Chemical Society 2002, 124 (7), 1424-1429.

72. Canham, L. T., Silicon Quantum Wire Array Fabrication by Electrochemical and Chemical Dissolution of Wafers. Applied Physics Letters 1990, 57 (10), 1046-1048.

73. Cullis, A. G.; Canham, L. T.; Calcott, P. D. J., The structural and luminescence properties of porous silicon. Journal of Applied Physics 1997, 82 (3), 909965.

74. Cullis, A. G.; Canham, L. T., Visible-Light Emission Due to Quantum Size Effects in Highly Porous Crystalline Silicon. Nature 1991, 353 (6342), 335-338.

75. Prokes, S. M., Surface and optical properties of porous silicon (vol 11, pg 305, 1996). Journal of Materials Research 1996, 11 (7), 1851-1851.

76. Chang, I. M.; Chen, Y. F., Light emitting mechanism of porous silicon. Journal of Applied Physics 1997, 82 (7), 3514-3518. 
77. Ozanam, F.; Chazalviel, J. N.; Wehrspohn, R. B., In-situ photoluminescence studies of porous silicon in liquids. Thin Solid Films 1997, 297 (1-2), 53-60.

78. Wells, A. F., Structural Inorganic Chemistry. Oxford University Press, USA; 5 edition: 1984.

79. Saito, Y., Crystal-Structure and Habit of Silicon and Germanium Particles Grown in Argon Gas. Journal of Crystal Growth 1979, 47 (1), 61-72.

80. Levinshtein M.E., S. L. R., Handbook Series on Semiconductor Parameters. World Scientific, London,: 1996.

81. Dutta, A. K., Visible photoluminescence from Ge nanocrystal embedded into a $\mathrm{SiO} 2$ matrix fabricated by atmospheric pressure chemical vapor deposition. Applied Physics Letters 1996, 68 (9), 1189-1191.

82. Kanemitsu, Y.; Uto, H.; Masumoto, Y.; Maeda, Y., On the Origin of Visible Photoluminescence in Nanometer-Size Ge Crystallites. Applied Physics Letters 1992, 61 (18), 2187-2189.

83. Niquet, Y. M.; Allan, G.; Delerue, C.; Lannoo, M., Quantum confinement in germanium nanocrystals. Applied Physics Letters 2000, 77 (8), 1182-1184.

84. Taylor, B. R.; Kauzlarich, S. M.; Lee, H. W. H.; Delgado, G. R., Solution synthesis of germanium nanocrystals demonstrating quantum confinement. Chemistry of Materials 1998, 10 (1), 22-24. 
85. Takeoka, S.; Fujii, M.; Hayashi, S.; Yamamoto, K., Size-dependent nearinfrared photoluminescence from Ge nanocrystals embedded in $\mathrm{SiO} 2$ matrices. Physical Review B 1998, 58 (12), 7921-7925.

86. Steiner, T. D., Semiconductor Nanostructures for Optoelectronic Applications Artech House Publishers: 2004.

87. Findley, W. R., Fluorescent Whitening Agents. Soap Cosmetics Chemical Specialties 1988, 64 (7), 44-\&.

88. Grancaric, A. M.; Tarbuk, A.; Dumitrescu, I.; Biscan, J., Influence of fluorescent whitening agents on ultraviolet protection of pretreated cotton. Aatcc Review 2006, 6 (4), 44-48.

89. Kaschig, J.; Schaumann, M.; Schultz, B., The photochemistry of fluorescent whitening agents: Impact on light fastness. Proceeedings of the 4th World Conference on Detergents: Strategies for the 21st Century 1999, 323-325

90. Bourgoing, S.; Robert, S., Use of fluorescent whitening agents against lightinduced colour reversion of high yield pulps. Iswpc - 9th International Symposium on Wood and Pulping Chemistry - Poster Presentations 1997, 101-104

91. Higginbotham, R. S.; Thomas, F. W., Fluorescent Whitening Agents. Nature 1958, 181 (4621), 1437-1439. 
92. Crouse, B. W.; Snow, G. H., Fluorescent Whitening Agents in the PaperIndustry - Their Chemistry and Measurement. Tappi 1981, 64 (7), 87-89.

93. Wang, S. G.; Yang, Q. B.; Du, J. S.; Bai, J.; Li, Y. X., Variety of photoluminescence intensity of fluorescent whitening agents introduced into polyacrylonitrile nanofibers. Journal of Applied Polymer Science 2007, 103 (4), 2382-2386.

94. Amirshahi, S. H.; Agahian, F., Basis functions of the total radiance factor of fluorescent whitening agents. Textile Research Journal 2006, 76 (3), 197-207.

95. Holt, L. A.; Leaver, I. H.; Milligan, B., Fluorescent Whitening Agents .8. Photochemical Behavior of Coumarin and Stilbene Whiteners in Polymer-Films and in Surface Whitened Wool. Textile Research Journal 1976, 46 (7), 539-544.

96. Holt, L. A.; Milligan, B.; Wolfram, L. J., Fluorescent Whitening Agents .6. Whitening of Wool by Surface Application of Whitener-Polymer Solutions. Textile Research Journal 1975, 45 (3), 257-264.

97. Holt, L. A.; Milligan, B., Fluorescent Whitening Agents .3. Photodecomposition of Disodium 4,4-Diacetamidostilbene-2,2-Disulfonate. Textile Research Journal 1974, 44 (3), 181-183.

98. Tenne, R.; Margulis, L.; Genut, M.; Hodes, G., Polyhedral and Cylindrical Structures of Tungsten Disulfide. Nature 1992, 360 (6403), 444-446. 
99. Feldman, Y.; Wasserman, E.; Srolovitz, D. J.; Tenne, R., High-Rate, GasPhase Growth of Mos2 Nested Inorganic Fullerenes and Nanotubes. Science 1995, 267 (5195), 222-225.

100. Nath, M.; Rao, C. N. R., Nanotubes of group 4 metal disulfides. Angewandte Chemie-International Edition 2002, 41 (18), 3451-3454.

101. Chen, J.; Li, S. L.; Tao, Z. L.; Shen, Y. T.; Cui, C. X., Titanium disulfide nanotubes as hydrogen-storage materials. Journal of the American Chemical Society 2003, 125 (18), 5284-5285.

102. Tao, Z. L.; Xu, L. N.; Gou, X. L.; Chen, J.; Yuan, H. T., TiS2 nanotubes as the cathode materials of Mg-ion batteries. Chemical Communications 2004, (18), 2080-2081.

103. Wilson, J. A.; Yoffe, A. D., Transition Metal Dichalcogenides Discussion and Interpretation of Observed Optical, Electrical and Structural Properties. $A d$ vances in Physics 1969, 18 (73), 193-\&.

104. Imai, H.; Shimakawa, Y.; Kubo, Y., Large thermoelectric power factor in TiS2 crystal with nearly stoichiometric composition. Physical Review B 2001, 64 (24).

105. Margolin, A.; Popovitz-Biro, R.; Albu-Yaron, A.; Rapoport, L.; Tenne, R., Inorganic fullerene-like nanoparticles of $\mathrm{TiS}_{2}$. Chem. Phys. Lett. 2005, 411, 162.

106. Park, K. H.; Choi, J.; Kim, H. J.; Oh, D.-H.; Ahn, J. R.; Son, S. Unstable 
single-layered colloidal $\mathrm{TiS}_{2}$ nanodisks. Small 2008, 7, 945.

107. Zhang, Y.; Li, Z.; Jia, H.; Luo, X.; Xu, J.; Zhang, X.; Yu, D., TiS ${ }_{2}$ crystal growth by a simple vapor-deposition method. J. Cryst. Growth 2006, 293, 124.

108. Tao, Y.-R.; Wu, X.-C.; Zhang, Y.-L.; Dong, L.; Zhu, J.-J.; Hu, Z., Thio-gel synthesis of titanium disulfide thin films and nanoparticles using titanium(IV) alkoxide precursors. Cryst. Growth Des. 2008, 8 ( 8) 2990.

109. Ma, J.; Jin, H.; Liu, X.; Fleet, M. E.; Li, J.; Cao, X.; Feng, S., Selective synthe sis and formation mechanism of TiS2 dendritic crystals. Cryst. Growth Des. 2008, 8 ( 12) 4460 .

110. Yang, Q.; Tang, K.; Wang, C.; Zhang, D.; Qian, Y., The synthesis of $\mathrm{SnS}_{2}$ nanoflakes from tetrabutyltin precursor. J. Solid State Chem. 2002, 164, 106.

111. David, J. S.; Pradeep, T.; Joydeep, B.; Umesh, W. V., Closed-cage clusters in the gaseous and condensed phases derived from sonochemically synthesized $\mathrm{MoS}_{2}$ nanoflakes. J. Am. Soc. Mass Spectrom. 2007, 18, 2191.

112. X. Liu, C. Lee, C. Zhou, and J. Han, Carbon nanotube field-effect inverters Appl. Phys. Lett. 79, 3329 (2001). 
113. Cao. G., Nanostructures and nanomaterials: Synthesis, Properties and Applications, Imperial college press.

114. Millington. K. T., Photoyellowing of wool. Part 2: Photoyellowing mechanisms and methods of prevention, Color. Technol, 122, 301-316.

115. Rosso-Vasic M., Synthesis and Photo physics of Functionalized Silicon Nanoparticles, PhD Thesis, Wageningen University, 2008.

116. Pavesi, L and Turan, R. Silicon Nanocrystals: Fundamentals, Synthesis and Applications, WILEY-VCH Verlag GmbH \& Co. KGaA, Weinheim, 2010 


\section{Characterization Techniques}

The synthesis of nanomaterials by solution phase techniques provides us with a plethora of methods for controlling size, morphology and functionality. ${ }^{1}$ The as-synthesized nanoparticles and nanostructures could be suspended in a variety of organic solvents depending on the attached functional groups or simply based on their solubilities. ${ }^{2}$ Purification of the nanomaterials is carried out mainly to remove excess surfactant, solvent, impurities and by-products, facilitating easy characterization. The assynthesized nanomaterials are then characterized using a variety of techniques; 3,4 however electron microscopy was used as the primary characterization tool. This chapter deals with the characterization techniques used in this research. A brief paragraph listing the various techniques that were used is given below followed by a more detailed explanation in the latter parts of the chapter.

High Resolution Transmission Electron Microscopy (HRTEM) studies provided detailed information on the structure and crystallographic orientation of the sample. Scanning Electron Microscopy (SEM) was used in this research to investigate the morphology and growth mechanism of titanium disulphide nanostructures studied in chapter 6. Selected Area Electron Diffraction (SAED) provides detailed information on the phase and orientation of the sample. Energy Dispersive X-ray Spectroscopy (EDX) used in conjunction with both scanning and transmission electron microscopy was used to determine the elemental composition of the samples. Powder X-Ray Diffraction Analysis (PXRD) was used when sufficient quantities of the sample were obtained and provided information on the crystallographic phases of a sample. 
Fourier Transform Infrared spectroscopy (FTIR) was used on nanoparticle samples primarily to investigate their surface functionality after chemical modification, whilst Ultraviolet-visible spectroscopy (UV-Vis) was used to determine the absorption of UVVis by a sample. Photoluminescence spectroscopy (PL) studies were conducted on fluorescing nanocrystal samples to determine the emission.

Techniques such as the B.E.T surface area measurement (Brunauer, Emmett, and Teller) for measuring the surface area of nanostructures, High Performance Liquid Chromatography (HPLC) to monitor singlet oxygen species (ROS) in nanocrystals and light meter measurements used to determine the brightness of nanoparticle modified fabrics were conducted in conjunction with some of the application oriented studies of the materials. It should be noted that the above three techniques are discussed quite briefly, mainly because of their minor significance to the scope of this research and the thesis.

\subsection{Electron Microscopy}

\subsubsection{Transmission Electron Microscopy}

Basics of TEM: The transmission electron microscope (TEM) is a very important tool that provides valuable structural and morphological information. The electron microscope contains an electron source which provides a beam of electrons by a process called thermionic emission (Edison effect). The charged nature of the electrons enables the beam to be controlled and focused by magnetic fields to generate diffraction patterns and image the materials. Figure 2.1 is a schematic of a transmission electron microscope column. The source of electrons is usually a tungsten filament or a lanthanum hexaboride $\left(\mathrm{LaB}_{6}\right)$ single crystal. The latter being the source used in our technique, is a 
standard self-biasing type with $\mathrm{LaB}_{6}$ emitter. The fast moving electrons are accelerated by a potential difference of $200 \mathrm{kV}$ and wavelengths $\left(\lambda=0.0251 \mathrm{~A}^{\mathrm{o}}\right)$ much smaller than the inter-atomic distances in crystal lattices. They are thus diffracted by the atoms due to electron-beam interactions of the crystal. Due to the relativistic effects on the electron caused by the acceleration by the large potential difference, the precise wavelength of the electrons is given by equation:

$$
\lambda=\frac{h\left(1-\beta^{2}\right)^{1 / 4}}{\left(2 m_{e} e_{0} E\right)^{1 / 2}} \quad \text { Eq. (2.1) }
$$

Where $\mathrm{h}$ is the plank's constant, $\mathrm{m}_{\mathrm{e}}$ is the rest mass of the electron, $\mathrm{e}_{0}$ is the electronic charge, $\mathrm{E}$ is the accelerating voltage and $\beta=\mathrm{v} / \mathrm{c}$, where $\mathrm{v}$ and $\mathrm{c}$ are the velocities of the electron and light respectively, in vacuum.

The electrons are first focused to form an approximately parallel beam by the condenser lenses. This beam then strikes the sample. A ray diagram between the specimen and the screen is shown in figure 2.2. Some of the electrons will be diffracted by the sample imparting structural information to the beam. The diffracted beam and the primary beam emerge through the sample and are recombined by the objective lens. The electron beam wave fronts can then be focused to a point which lies on a plane, normal to the straightthrough beam, which is the back focal plane of the objective lens. This produces an electron diffraction pattern. In effect the objective lens produces a Fourier transform of the electron density in the crystal. The other lenses complete an inverse Fourier transform to give an 'image'. By alteration of the projector lenses either the image of the 
electron diffraction pattern can be propagated down the microscope and be recorded on a fluorescent screen, photographic plate or captured by a CCD camera. 


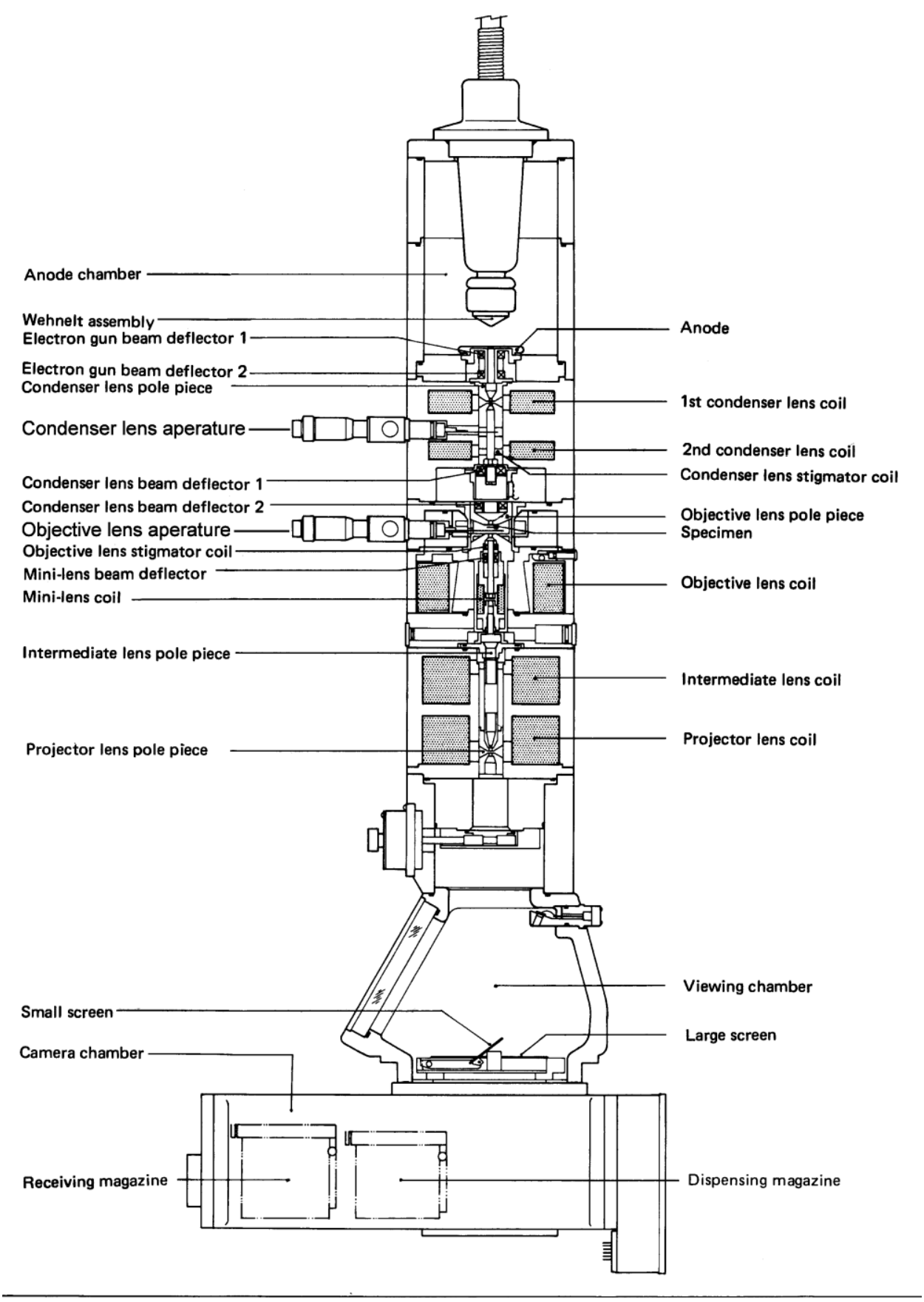

Figure 2.1: Schematic of a transmission electron microscope 


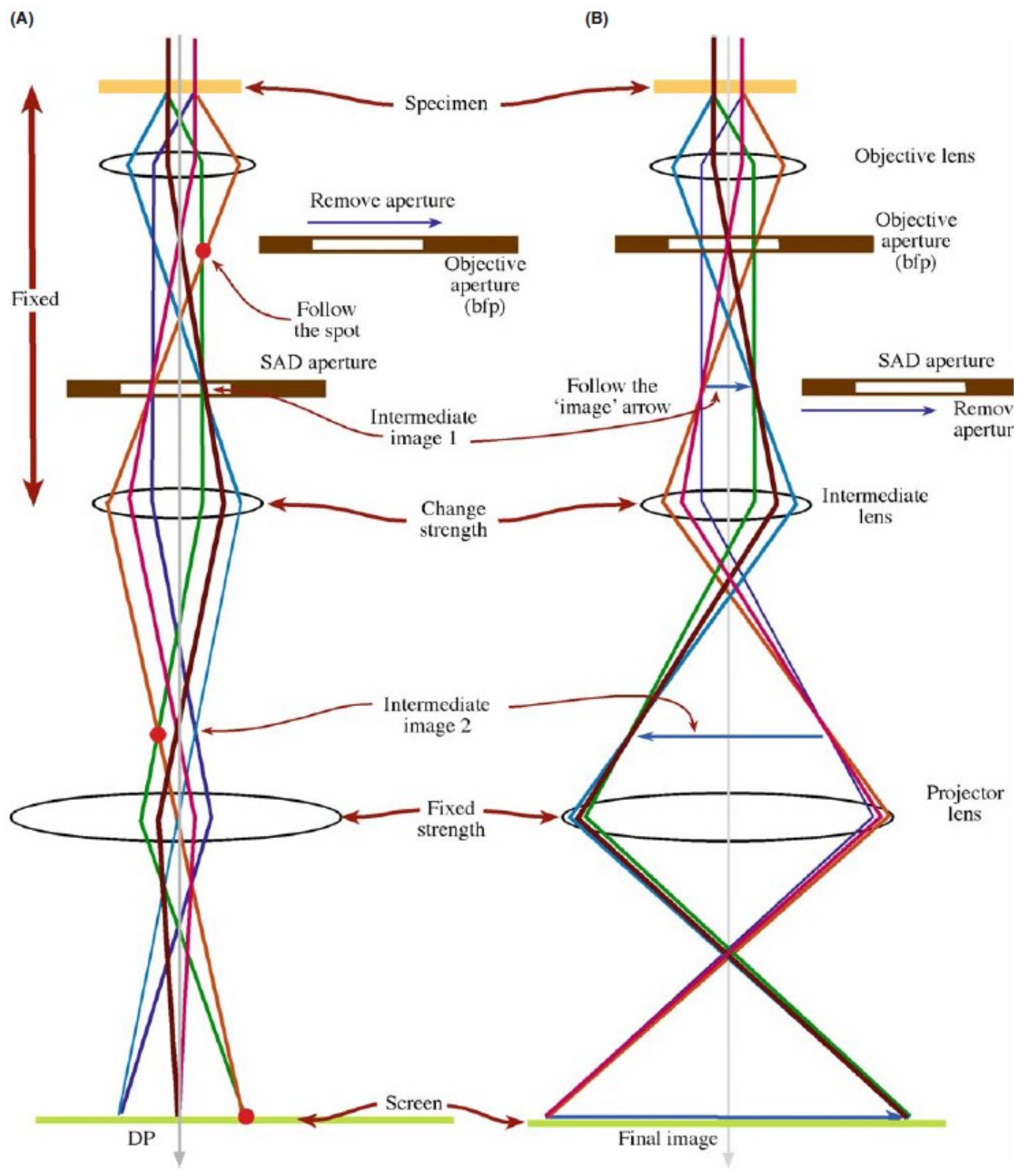

Figure 2.2: Ray diagram of a transmission electron microscope in (A) diffraction mode: projecting the diffraction pattern onto the viewing screen and (B) image mode: projecting the image onto the screen. (Obtained from William and Carter) 


\subsubsection{High-Resolution TEM (HRTEM) imaging}

The structural information of crystalline nanomaterials could be directly obtained using HRTEM. Although detailed imagery could be obtained using this technique, one should be aware of the constraints that the TEM could impose on the information of the image, since a TEM image is basically formed by the recombination of diffracted and undiffracted electron beams. Such constraints are usually caused by the objective lens. The objective lens recombines the diffracted beams from the sample to form the initial image. Any imperfections in this lens such as astigmatism and spherical aberration must be considered. Electromagnetic lenses within the microscope can be used to correct the degree and orientation of the microscope, caused by the departure of the lens bore from a cylindrical nature. Theoretically these coils should allow the complete correction of astigmatism and is not found to be a problem in microscopes used these days. The actual lenses in a microscope are the magnetic field within the vacuum between the pole-pieces of the objective lenses and the geometry of this magnetic field is difficult to control. This causes the beams which are diffracted though different angles to be brought into focus at slightly different planes. Such a defect is known as spherical aberration and can be corrected in optical lenses by making them aspherical. However this cannot be done in magnetic lenses and could be only countered to some effect by increasing the focal length, whereby the focus is adjusted by an amount $\Delta \mathrm{F}$, which is referred to as the defocus. The spherical aberration coefficient of the objective lens, $\mathrm{C}_{\mathrm{s}}$ and the defocus $\Delta \mathrm{F}$ are related by the phase contrast transfer function, $\mathrm{CTF}(\mathrm{u})$, given by 
$\mathrm{CTF}(\mathrm{u})=\mathrm{A}(\mathrm{u}) \mathrm{E}(\mathrm{u}) \sin (\chi(\mathrm{u})) \quad$ Eq. (2.2)

Where $\mathrm{A}(\mathrm{u})$ is the aperture function, $\mathrm{E}(\mathrm{u})$ the envelope function, $\mathrm{u}$ a reciprocal lattice vector, and $\chi(\mathrm{u})$ the phase distortion function, given by

$$
\chi(u)=\frac{\pi}{2} C_{s} \lambda^{3} u^{4}-\pi \Delta f \lambda u^{2} \quad \text { Eq. (2.3) }
$$

The introduction of phase shifts into the diffracted beams is described by CTF (u), which converts the phase difference into an amplitude difference in the image intensity. CTF $(\mathrm{u})$ is at maxima when $\chi(\mathrm{u})$ is a multiple of $\pm \pi / 2$ and at zero when $\chi(\mathrm{u})$ is a multiple of $\pm \pi$. Thus, the phase shift of electron wave determines the appearance of a high resolution TEM image. At negative $\mathrm{CTF}(\mathrm{u})$, the phase contrast is positive and whilst viewing a brighter background, the atoms will appear darker. The opposite is observed, when CTF $(\mathrm{u})$ is positive and no detail when CTF $(\mathrm{u})$ is zero.

When $\Delta \mathrm{F}$ and $\mathrm{C}_{\mathrm{s}}$ are opposite, there is a value of defocus where $\Delta \mathrm{F}$ and $\mathrm{C}_{\mathrm{s}}$ cancel out known as the Scherzer defocus, $\Delta \mathrm{F}_{\text {Sch. }}$ In the Scherer defocus one aims to counter the term $\mathrm{u}^{4}$ with the parabolic term $\Delta \mathrm{fu}^{2}$ of $\chi(\mathrm{u})$, creating a wide band where low spatial frequencies $\mathrm{u}$ are transferred to image intensity with a similar phase. The Scherer defocus occurs at

$$
\Delta \mathrm{F}_{\mathrm{Sch}}=-1.2\left(\mathrm{C}_{\mathrm{s}} \lambda\right)^{1 / 2} \quad \text { Eq. (2.4) }
$$


At this defocus all the beams have constant phase and the same relative intensities. This designates the resolution limit of the transmission electron microscope and typically lies within the range of 1.6-2.0 $\mathrm{A}^{\circ}$.

The other two important defects that affect TEM resolution are chromatic aberration and beam divergence. Chromatic aberration occurs mainly due to the fact that the focal length of the magnetic lens is dependent on the energy of the electrons. The electron beam which is known to have a spread of energies and consequently wavelengths is not perfectly monochromatic. The fluctuations in the accelerating voltage and the objective lens current and the inherent thermal energy spread of electrons emitted from the electron source would also additionally contribute to the occurrence of chromatic aberration. Beam divergence results from the filament not being a point source and consequently the incident beam is never exactly parallel. Both chromatic aberration and beam divergence reduce the performance of the microscope by introducing an uncertainty in the phase introduced by the objective lens.

A JEOL 2010 HRTEM operated at $200 \mathrm{keV}$ was used for TEM and HRTEM analyses. TEM samples were prepared by drying drops of solutions containing the nanomaterials on a TEM grid, utilizing either strong carbon films on 200 mesh copper or holey carbon films, purchased from ProSciTech. Whilst GATAN Digital Micrograph software suite was used to obtain digital images and to perform Fast Fourier transforms (FFT) on images, crystal structure models and simulated power spectrums were generated using CARINE. 


\subsubsection{Data Interpretation:}

Using HRTEM highly detailed images of atomic resolution could be obtained. A crystal whose atoms are highly ordered could be distinguished from the carbon film of the TEM grid, because of its amorphous nature. For example in figure 2.3a, a germanium nanocrystal with discernible lattice fringed is shown. Using the image, a power spectrum (inset) was calculated from a Fast Fourier transform (FFT) of the image (figure 2.3b). Figure $2.3 \mathrm{~b}$ exhibits the characteristic spot pattern of a single-crystal domain and the hexagonal symmetry of the [111] lattice planes of Ge. The role of a FFT is to transform a function from a spatial domain to a frequency domain. The power spectrum matches the simulated pattern calculated for cubic Ge viewed down the [111] direction. Lattice fringes, repeat themselves, and would show up as spots corresponding to the frequency of repetition. When all the inter-planar angles measured between spots in the power spectrum and those measured in the simulated diffraction pattern match, and the values of atomic plane spacing matches that of the reference, a perfect match is said to be made. This method was done routinely in order to facilitate proper characterization of the sample. 


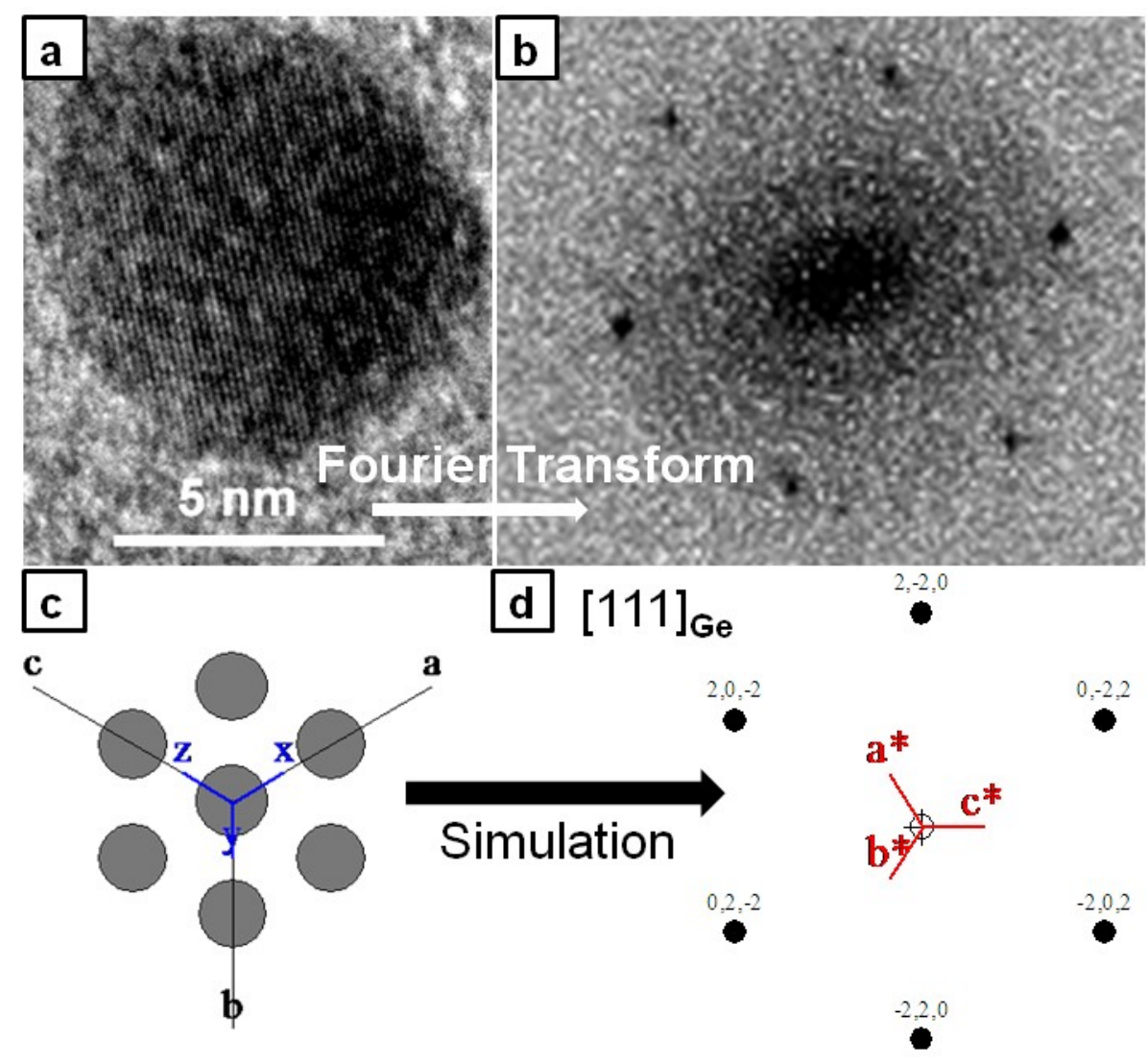

Figure 2.3: (a) HRTEM image of an germanium nanocrystal; (b) a power spectrum; (c) a unit cell of cubic Ge looking down the [111] direction and (d) the simulated diffraction pattern. 


\subsubsection{Resolution Limits in the Transmission Electron}

\section{Microscope}

While in principle, it should be possible to image atom arrangements on crystal surfaces with the same resolution as for the bulk of the crystals; practical limitations are imposed by the strong scattering by the bulk material. Diffraction and interference could be said to one of these causes. In transmission electron microscopy (TEM) severe limitations are imposed by the non-linearity of the variation of contrast with thickness or the need to image with reflections given only by the surface but not by the bulk crystal.

Even in a perfect optical system, a point of light cannot be focused as a perfect dot. Instead, the image when viewed critically consists of concentric circles with diminished intensity, known as an Airy disc. ${ }^{9}$

Resolution in a perfect optical system can be described mathematically by Abbe's equation.

$\mathrm{d}=0.612 * 1 / \mathrm{n} \sin \mathrm{a}-----$ Eq. $\mathbf{( 2 . 5 )}$

where:

$d=$ resolution

$1=$ wavelength of imaging radiation

$n=$ index of refraction of medium between point source and lens, relative to free space

$\mathrm{a}=$ half the angle of the cone of light from specimen plane accepted by the objective (half aperture angle in radians)

$n \sin$ a is often expressed as NA (numerical aperture) 
This is the diffraction-limited resolution of an optical system. If all aberrations and distortions are eliminated from the optical system, this will be the limit to resolution. If aberrations and distortions are present, they will determine the practical limit to resolution. ${ }^{9}$

De Broglie equation. By combining some of the principles of classical physics with the quantum theory, de Broglie proposed that moving particles have wave-like properties and that their wavelength can be calculated, based on their mass and energy levels. ${ }^{9}$ The general form of the de Broglie equation is as follows:

$\lambda=\mathrm{h} / \mathrm{m}^{*} \mathrm{v} \quad$ Eq. (2.6)

where:

$$
\begin{aligned}
& \lambda=\text { wavelength } \\
& h=\text { Planck's constant }\left(6.6 \times 10^{-27}\right) \\
& m=\text { mass of the particle }\left(9.1 \times 10^{-28}\right) \\
& v=\text { velocity of the particle }
\end{aligned}
$$

When an electron passes through a potential difference (accelerating voltage field) V, its kinetic energy with be equal to the energy of the field, i.e. eV (energy in electron volts) $=\mathrm{V}$ (the accelerating voltage). As you may recall, $\mathrm{e}=\mathrm{mc}^{2}$. By restating this for velocities below the speed of light and particles with true mass, the energy of an electron may be stated as follows:

$$
\mathrm{eV}=1 / 2 m v^{2} \ldots \mathbf{E q} \cdot(\mathbf{2 . 7 )}
$$

where:

$$
\begin{aligned}
& \mathrm{eV}=\text { energy in electron volts }\left(e=4.8 \times 10^{-10}\right) \\
& m=\text { mass of the particle } \\
& v=\text { velocity of the particle }
\end{aligned}
$$


By using some assumptions about the velocity of the particle and its mass, it is possible to express either wavelength (l) or velocity (v) in terms of the accelerating voltage (V). By further substituting the values of $h$ and $m$ above, the equation for 1 reduces to the following:

$$
\lambda=1.23 \mathrm{~nm} / \mathrm{V}^{1 / 2}-\mathbf{- - .} \text { Eq. (2.8) }
$$

Equation for resolution in TEM: This value for 1 can then be substituted into Abbe's equation. Since angle a is usually very small, for example $10^{-2}$ radians (a likely figure for TEM), the value of a approaches that of sin a, so we replace it. ${ }^{9}$ Since $n$ (refractive index) is essentially 1 , we eliminate it, and we multiply 0.612 by 12.3 to obtain 0.753 . Therefore, the equation reduces to the following:

$$
d=0.753 / a V^{1 / 2}-\mathbf{E q} \cdot \mathbf{( 2 . 9 )}
$$

where:

$$
\begin{aligned}
& d=\text { resolution in } \mathrm{nm} \\
& \alpha=\text { half aperture angle } \\
& \mathrm{V}=\text { accelerating velocity }
\end{aligned}
$$

Now, solving for 100,000 volts, the result is $0.24 \mathrm{~nm}$ or $2.4 \AA$. This improves with higher accelerating voltage and gets worse with lower voltages. (Using Einsteinian calculations, the resolution is: $0.22 \mathrm{~nm}$ or $2.2 \AA$.) Each lens and aperture has its own set of aberrations and distortions. ${ }^{9}$ If aberrations and distortions are present, they will determine the practical limit to resolution. 


\subsubsection{Selected Area Electron Diffraction:}

Selected area electron diffraction (SAED) analysis was used extensively in this research, to obtain crystal structures and structural data from the crystalline nanomaterials under study. One of the greatest advantages of the transmission electron microscope is its capability to observe, by adjusting the electron lenses, both electron microscope images (information in real space) and diffraction patterns (information in reciprocal space) for the same region. By inserting a selected area aperture and using the parallel incident beam illumination we get a diffraction pattern of an area as small as $100 \mathrm{~nm}$ in diameter. A diffraction pattern is obtained by placing an aperture in the imaging plane of the objective lens. Only rays passing through this aperture contribute to the diffraction pattern at the far field. The combination of imaging and diffraction in SAED mode makes it particularly useful for setting diffraction conditions for electron imaging in a TEM, such as lattice images or diffraction contrast. It is also one of the major electron microscopy techniques for identifying phases and determining the orientation of materials.

Interpretation of a Selected Area Diffraction Pattern: Whilst amorphous materials would give rise to broad and diffuse ring patterns, crystalline materials would produce well defined rings or discrete spots. After indexing the diffraction pattern of the crystalline material, the structure and orientation of the crystal could be found out. SAED patterns were obtained on electron microscopy films, and after developing the films, the

distance between symmetrically related diffraction spots (usually single crystalline nanomaterials), or the diameter of the rings (group of crystals), could be directly measured on the negative film. The diffraction radius, $\mathrm{R}$ is related to the Bragg angle, $\theta$ by 


$$
\frac{\mathrm{R}}{\mathrm{L}}=\tan 2 \theta \sim \theta
$$

Where, $\mathrm{L}$ is the camera length.

In the current study a constant camera length of $150 \mathrm{~cm}$ was used. This value can be altered by increasing or decreasing the magnification of the lenses, and is rather a calculated value than a physical distance. Since the Bragg angle, $\theta$ obtained from electron diffraction is very small, $\tan 2 \theta \approx 2 \theta \approx 2 \sin \theta$ is valid. Substituting the above and equation 2.5 into the Bragg's equation, gives

$$
\frac{\mathrm{R}}{\mathrm{L}}=\frac{\lambda}{\mathrm{d}}
$$

Or,

$$
d=\frac{2 \lambda L}{D}
$$

Where $\mathrm{D}$, is the diameter of the diffraction ring

The interplanar spacing can be calculated from this value, and to avoid uncertainties that might arise from $\lambda$, the term $2 \lambda \mathrm{L}$ was determined by calibration from the diffraction pattern of gold nanocrystals. 


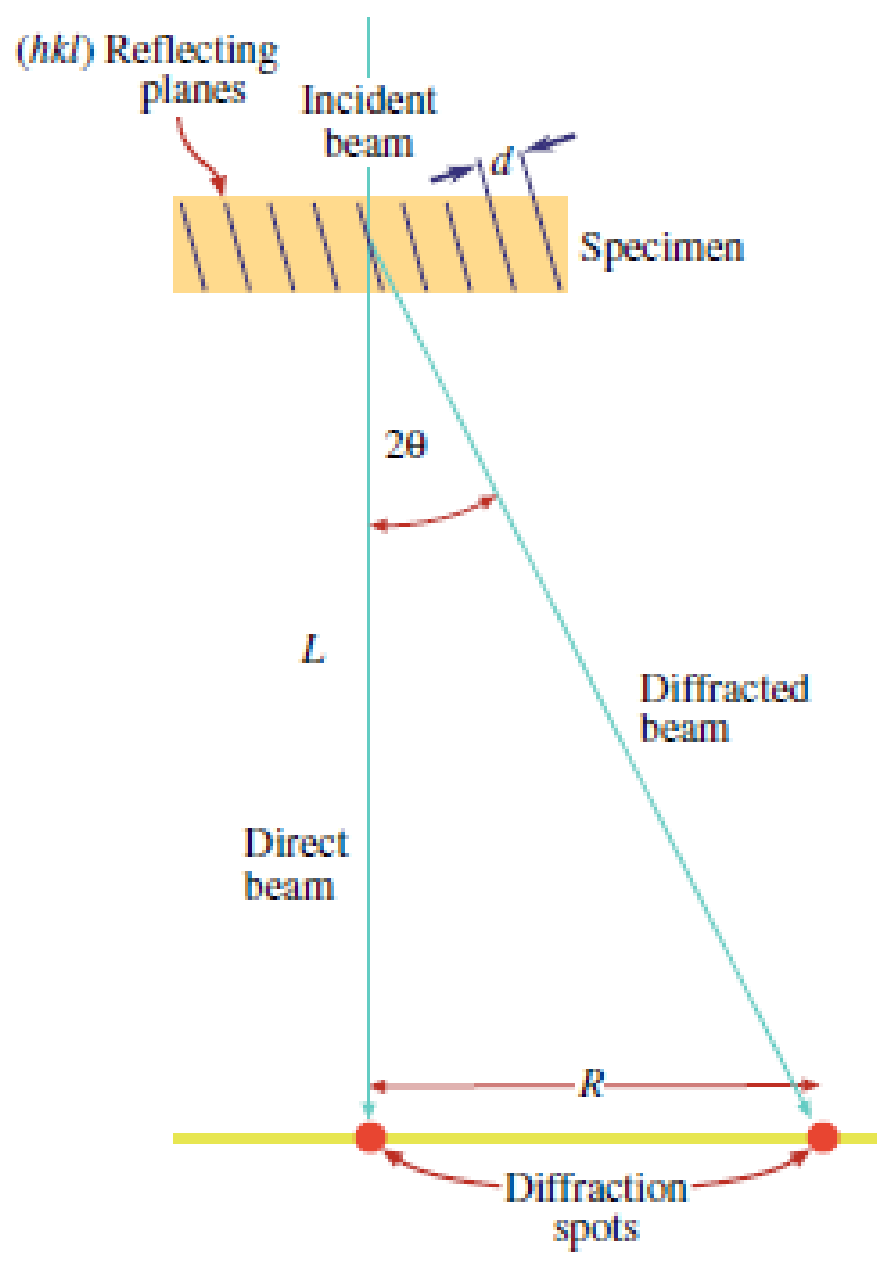

Figure 2.4: A Schematic representation of the relationship between camera length, Bragg angle and the diameter of the diffracted ring in a diffraction pattern. 


\subsubsection{Energy Dispersive X-ray Spectrometry (EDS)}

Energy dispersive X-ray spectroscopy (EDS) is a very important micro analytical technique that was used in conjunction with both the TEM and SEM in this current research. It uses the characteristic X-rays generated from a sample bombarded with electrons to identify the elemental constituents comprising the sample. A spectrum corresponding to specific X-ray lines and elements are generated, and can be easily identified. Quantitative data can also be obtained by comparing peak heights or areas in the unknown with a standard material. Data collection and analysis with EDS is a relatively quick and simple process because the complete spectrum of energies is acquired simultaneously.

When a beam of high energy electrons bombard the sample, X-rays are generated. Two processes can occur when an electron strikes an atom; elastic and inelastic scattering. While the former is used in forming images, the latter is used in EDS. The electrons that are generated by the microscope are accelerated through 200 kilovolts, thus containing sufficient energy to ionize atoms by removing an electron from one of the core shells, before continuing on with reduced energy. If the electron ejected is from an inner shell then an electron from an outer shell will fall to remove the vacancy thus stabilizing the atom. Energy is lost when an electron drops from a higher energy shell to a lower energy shell and this could be either due to the emission of a second electron or an Xray photon. This energy is characteristic of the energy level separation and can be used to identify the elements present within a crystal, since it is unique to each element. 
However in energy dispersive spectroscopy X-ray photons are detected rather than the Auger electrons.

The emitted X-rays have energies that are characteristic of the shells involved and are their transitions have specific terminologies. An $\mathrm{L}$ to $\mathrm{K}$ transition is given by $\mathrm{K}_{\alpha}$ and an $\mathrm{M}$ to $\mathrm{K}$ transition by $\mathrm{K}_{\beta}$.

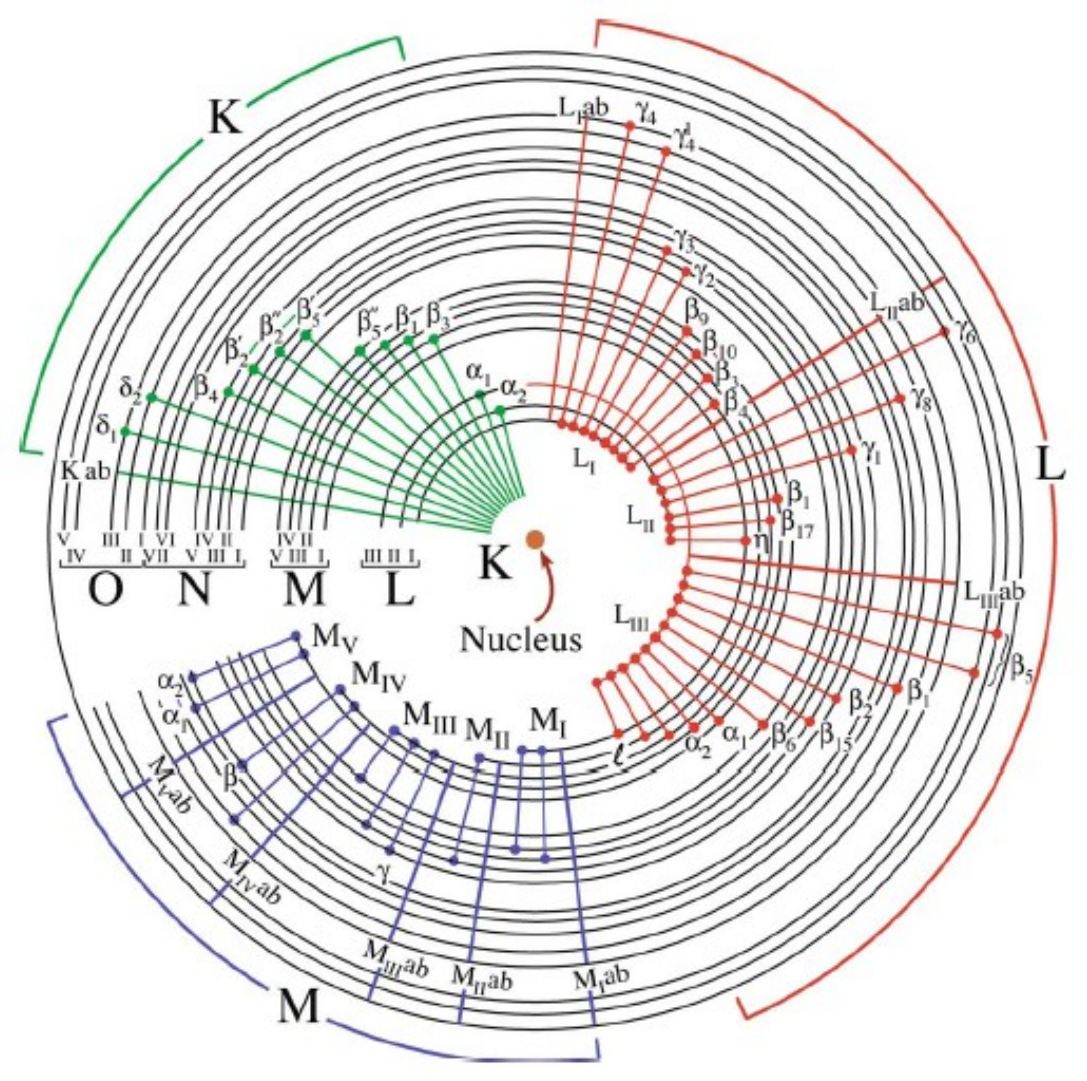

Figure 2.5: The complete range of possible electron transitions that give rise to K, L, M characteristic X-rays. Not all X-rays are detectable by EDS in the TEM. (Courtesy David B. Williams and Carter, C. B. ${ }^{4}$ )

When X-rays are generated by a crystal, there is a possibility that they could be generated deep in a thick crystal and be absorbed by the rest of the crystal. This wavelength 
dependent absorption is in turn dependent on the element and could be a source of misleading data. However this limitation can be dealt with, by using thin, crystals thus limiting sample re-absorption. The X-ray detector in the electron microscope contains a crystal of silicon doped with lithium and absorbs X-rays, thus creating an electron hole pair which in turn induces a current. The detector can process only a certain amount of $\mathrm{X}$-rays at a given time and if the input is too much, it would tend to detect emissions of lower energy compared to higher energy emissions. This again, results in erroneous data and could be avoided by using thin

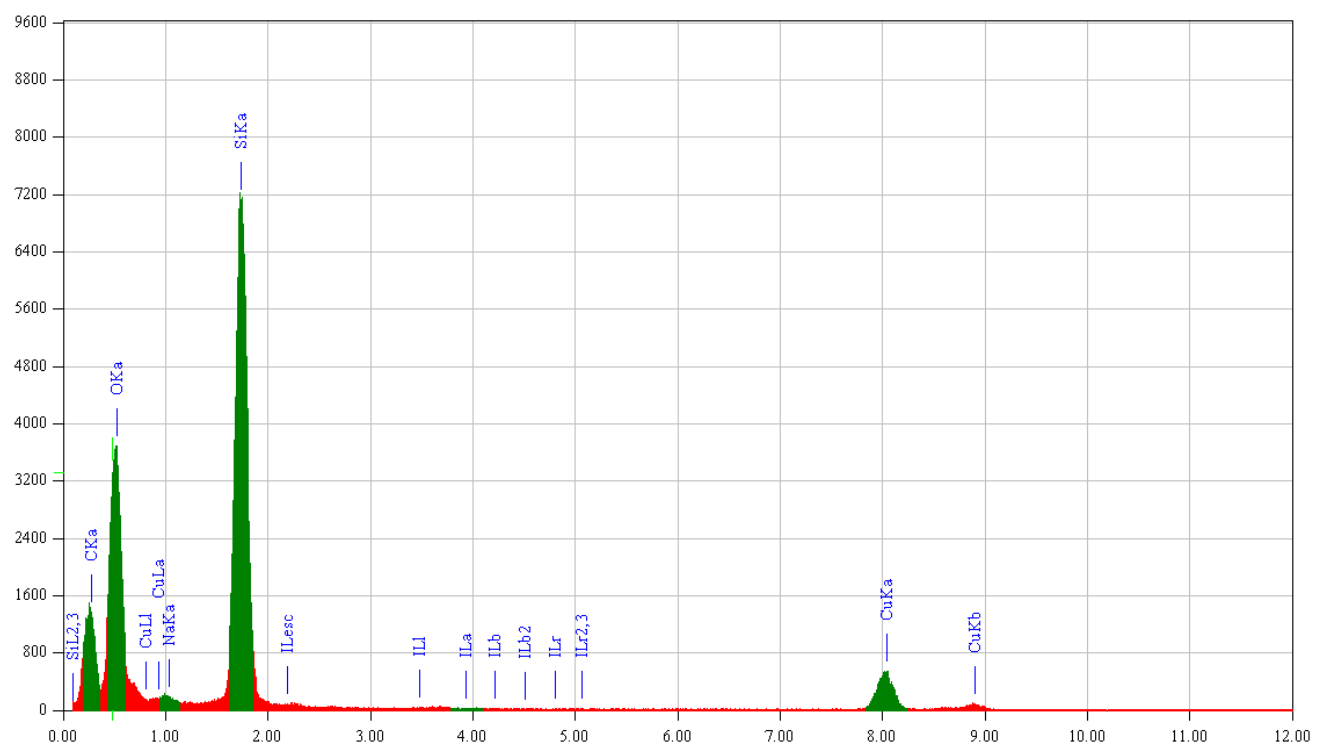

Figure 2.6: An energy dispersive spectroscopy spectrum of silicon nanocrystals

crystals and also by reducing the beam current, so that the numbers of incident electrons on the specimen are reduced. An EDX spectrum of nanocrystalline silicon is shown in the figure2.9. With a carbon coated copper grid, X-ray emission from carbon and copper 
are observed. Heavier elements like tin would give a higher number of transitions compared to lighter elements like carbon.

\subsubsection{Scanning Electron Microscopy (SEM)}

Basics of SEM: The SEM permits the observation of materials in macro and submicron ranges. The instrument is capable of generating three-dimensional images for analysis of topographic features. In the SEM, a beam of electrons is focused on a spot volume of the specimen, resulting in the transfer of energy to the spot. These bombarding electrons, also referred to as primary electrons, dislodge electrons from the specimen itself. These, also known as secondary electrons, are attracted and collected by a positively biased grid or detector, and then translated into a signal. To produce an SEM image, the electron beam is swept across the area being inspected, producing many such signals. These signals are then amplified, analyzed, and translated into images of the topography being inspected. Finally, the image is shown on a computer screen. ${ }^{6 \mathrm{~b}}$

The energy of the primary electrons determines the quantity of secondary electrons collected during inspection. The emission of secondary electrons from the specimen increases as the energy of the primary electron beam increases, until a certain limit is

reached. Beyond this limit, the collected secondary electrons diminish as the energy of the primary beam is increased, because the primary beam is already activating electrons deep below the surface of the specimen. Electrons coming from such depths usually recombine before reaching the surface for emission. Aside from secondary electrons, the primary electron beam results in the emission of backscattered (or reflected) 
electrons from the specimen. Backscattered electrons possess more energy than secondary electrons, and have a definite direction. As such, they can not be collected by a secondary electron detector, unless the detector is directly in their path of travel. All emissions above $50 \mathrm{eV}$ are considered to be backscattered electrons.

Backscattered electron imaging is useful in distinguishing one material from another, since the yield of the collected backscattered electrons increases monotonically with the specimen's atomic number. Backscatter imaging can distinguish elements with atomic number differences of at least 3 , i.e., materials with atomic number differences of at least 3 would appear with good contrast on the image. ${ }^{6 b}$ 


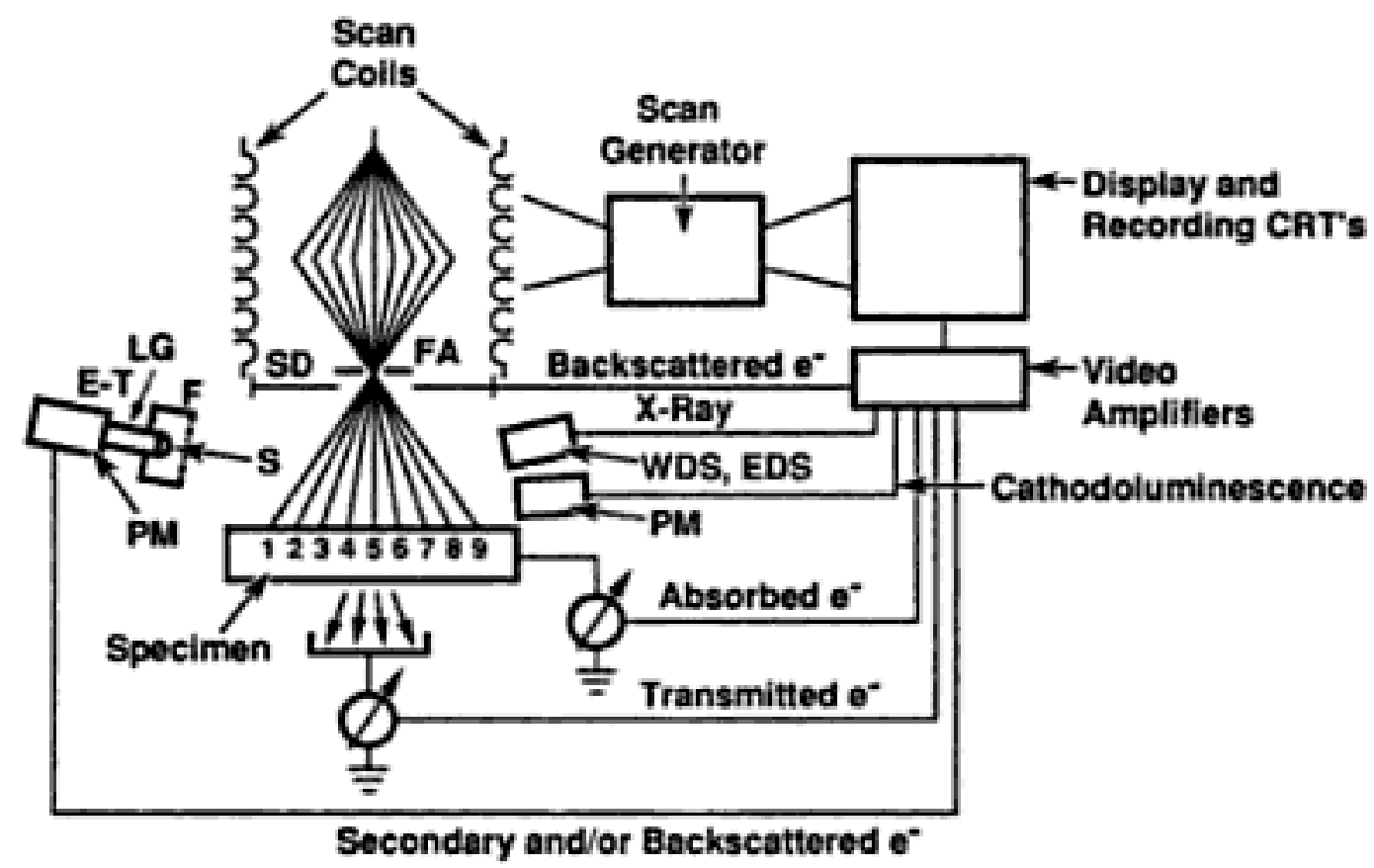

Figure 2.7: A schematic illustration of the scanning system of the SEM. The abbreviations are : FA, final aperture; SD, solid state back scattered electron detector; EDS, energy dispersive $\mathrm{x}$-ray spectrometer; WDS, wavelength-dispersive $\mathrm{x}$-ray spectrometer; CRT, cathode ray tube; E-T, Everhart-Thornley secondary/backscattered electron detector, consisting of F, Faraday cage; S, Scintillator; LG, light guide; and PM, photomultiplier.

From the illustration (figure 2.7) it can be seen that the electron beam, exits the electron column, enters the specimen chamber, and strikes the specimen at a single location on the optical axis of the column. The beam electrons interact elastically and in-elastically with the specimen, forming the limiting interaction volume from which various types of radiation emerge, including backscattered, secondary and absorbed electrons. By recording the magnitude of the signals with suitable detectors, a measure of certain 
properties of the specimen, such as topography, composition, etc. can be made at the single location where the electron beam strikes. ${ }^{6 a}$

High resolution scanning electron microscopy images were obtained using a JSM-6500F SEM. Samples were prepared by suspending the material in a suitable solvent and a small drop of the solvent dried on a silicon substrate. According to the material used, the materials were either coated with platinum or carbon to prevent charging. Figure 2.8 is a typical SEM image of a nanostructured material $\left(\mathrm{TiS}_{2}\right)$ illustrating the rather exotic three-dimensional qualities of the material.

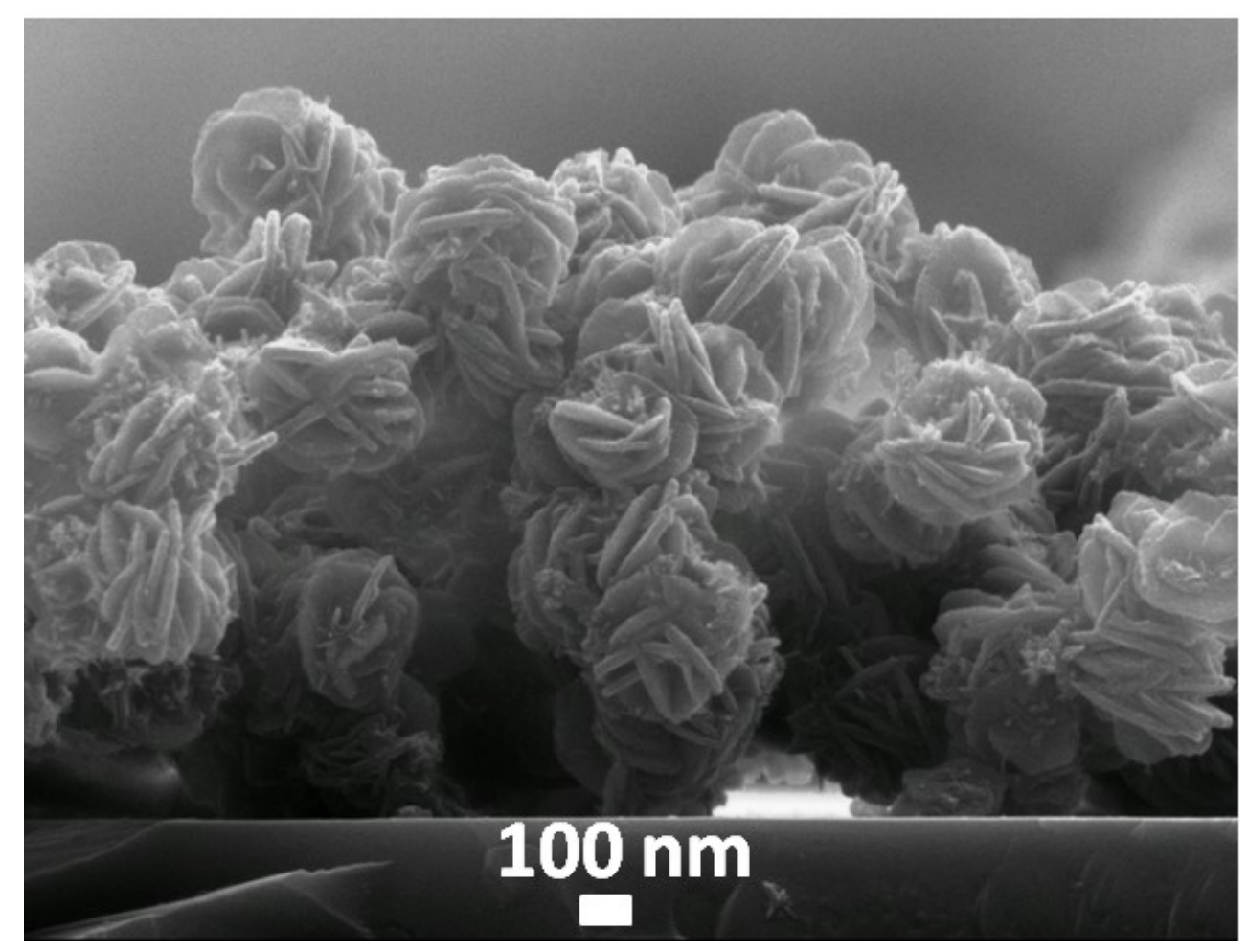

Figure 2.8: An SEM image of nanostructured $\mathrm{TiS}_{2}$. 


\subsection{Powder X-ray Diffraction (PXRD)}

Powder X-ray diffraction analysis is useful in providing structural information of crystalline materials that diffract when illuminated with an X-ray beam. A Detailed text on the basic principles of the technique could be obtained from Billinge and Dinnebier ${ }^{5}$.

Theory: X-ray diffraction analysis involves the measurement of the intensity of X-rays scattered from electrons bound to atoms. X-rays are electromagnetic waves whose wavelengths are typically in the range of $\sim 0.1-100 \AA$. These waves are scattered in all directions for a given scattering angle. As crystals are made of repeating units, a regular array of scattered waves is produced. The constructive interference of these waves gives rise to diffraction patterns consisting of specific directions. Such a condition is only possible for a given interplanar spacing $\mathrm{d}_{\mathrm{hkl}}$. Figure 2.9 illustrates the Braggs model of reflection of X-rays from a set of parallel planes. ${ }^{5}$

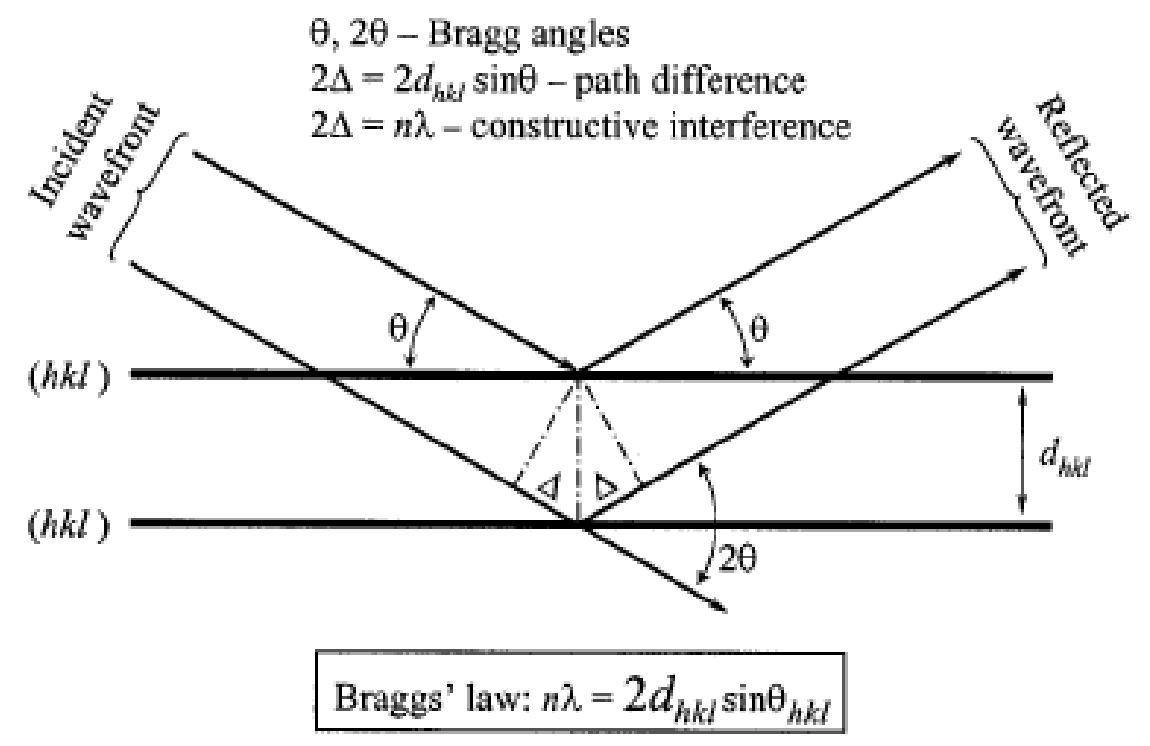

Figure 2.9: Geometrical illustration of the Braggs law. 
According to the Bragg's model diffraction from a crystalline sample can be explained by using a simple notion of mirror reflection of the incident X-ray beam from a series of crystallographic planes. Consider an incident front of waves with parallel propagation vectors, which form an angle $\theta$ with the planes (hkl). In a mirror reflection, the reflected wavefront will also consist of parallel waves, which form the same angle $\theta$ with all planes. The path differences introduced between a pair of waves both before and after they are reflected by the neighbouring planes, A, are determined by the interplanar distance as $\mathrm{A}=\mathrm{d}_{\mathrm{hk} \mid} \sin \theta$. The total path difference is $2 \Delta$, and the constructive interference is observed when $2 \Delta=\mathrm{n} \lambda$, where $\mathrm{n}$ is integer and $\lambda$ is the wavelength of the incident wavefront. This simple geometrical analysis results in the Braggs' law:

\section{$2 d_{h k l} \sin \theta_{h k l}=n \lambda$}

The integer $n$ is known as the order of reflection. Its value is taken as 1 in all calculations, since orders higher than one $(\mathrm{n}>1)$ can always be represented by first order reflections $(n=1)$ from a set of different crystallographic planes with indices that are multiples of $n$ because

$$
d_{h k l}=n d_{n h, n k, n l}
$$

and for any $\mathrm{n}>1$, Eq. 2.8 is transformed as follows:

$$
2 d_{h k l} \sin \theta_{h k l}=n \lambda \Rightarrow 2 d_{n h, n k, n l} \sin \theta_{h k l}=\lambda \quad \text { Eq. (2.10) }
$$

For a single crystal with a given orientation to the incident X-ray beam, only a few reflections might result, as the set of planes with interplanar spacing satisfying the Bragg 
law is limited. In order to observe all possible reflections, the orientation of the crystal with respect to the incident beam must be systematically varied to bring all crystallographic planes sequentially into diffraction conditions. A typical X-ray diffractogram is usually displayed as a line graph of intensity versus $2 \theta$, as shown in figure 2.10 .

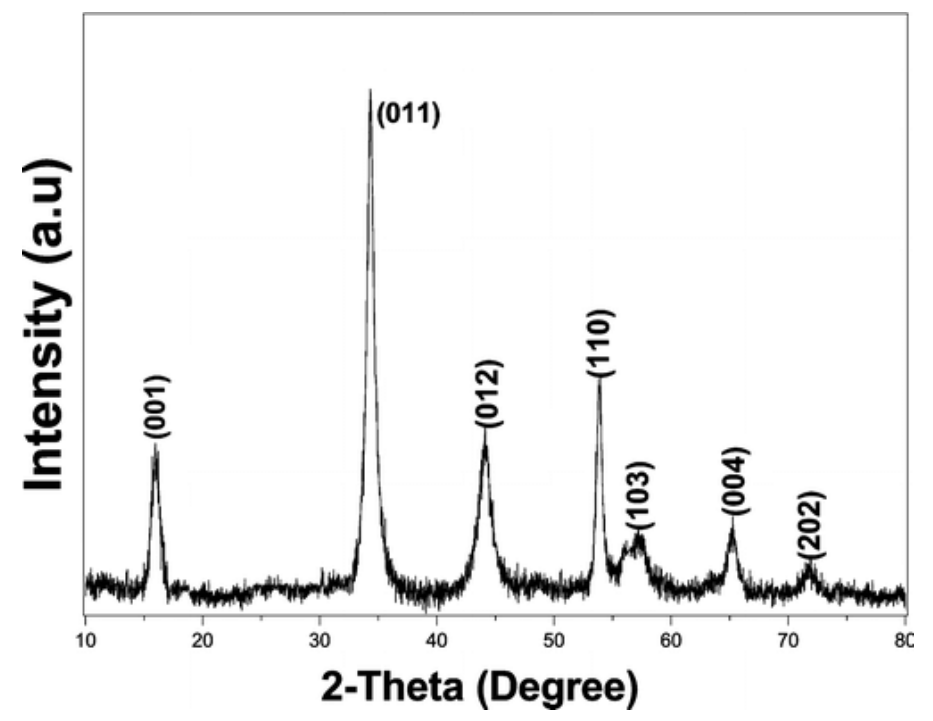

Figure 2.10: An example of a diffractogram

The intensity of X-ray reflections is dependent on parameters such as beam intensity of the X-ray source, the geometry of the diffractometer and the degree of adsorption of the specimen. This intensity is usually normalized and given as a relative intensity, given by

$$
F(h k l)=\sum_{j=1}^{n} f_{j, \theta} \operatorname{Exp}\left[i 2 \theta\left(h x_{j}+k y_{j}+l z_{j}\right)\right] \quad \text { Eq. (2.11) }
$$

Where, $\mathrm{F}(\mathrm{hkl})$ is the structure factor of a set of planes with indices hkl, the sum of scattering contributions from all $\mathrm{n}$ atoms within the unit cell. The contribution of the $j$ th atom is the product of its atomic scattering factor $\mathrm{f}_{\mathrm{j}, \theta}$ and an exponential term containing its fractional coordinates within the unit cell. ${ }^{5}$ 


\subsubsection{Sample Preparation and Data interpretation:}

Powder X-ray diffraction patterns were acquired on a Philips PW 1720 X-ray generator using $\mathrm{Cu} \mathrm{K \alpha}$ radiation. The data was processed using X'pert Data Collector software. A detector present in the diffractometer collects diffracted X-rays at a particular range of $2 \theta$ measured in a range between $20-80^{\circ}$. A tension of $40 \mathrm{kV}$ and a current of $20 \mathrm{~mA}$ were used for measurements. The nanomaterial samples were prepared by drying a suspended solution of the nanomaterials on a sample holder depending on the solvents reactivity with the sample holder, or the sample is dried separately under $\mathrm{N}_{2} /$ vacuum conditions and transferred to a sample holder. The instrument is calibrated a using polycrystalline silicon crystal to an uncertainty of $0.02^{\circ}$ in values of $2 \theta$. Information on the crystallite size was obtained by calculating the X-ray correlation length (L) from the fwhm of the XRD peak using the Scherer equation ${ }^{5}$ :

$$
\bar{D}=\frac{57.3 k \lambda}{\beta \cos \theta}
$$

Where $D=$ mean diameter, $k=$ shape factor, $\lambda=$ wavelength, $\beta=$ the full-width at half maximum, and $\theta=$ Bragg angle for studied ring. The factor 57.3 is used for conversion of $\beta$ from degree to radians. The Scherer equation, (2.12), predicts crystallite thickness if crystals are smaller than $100 \mathrm{~nm}$. Since small angular differences in angle are associated with large spatial distances (inverse space), broadening of a diffraction peak is expected to reflect some large scale feature in the crystal. The simplest way to obtain the Scherer equation is to take 
the derivative of Bragg's law holding the wavelength constant and allowing the diffraction angle and the Bragg spacing to vary, $2 d \sin \theta=\lambda$. Taking derivative in $d$ and $\theta$ yields $2 \Delta d \cos \theta \Delta \theta=\lambda$ since $\Delta \theta$ can be positive or negative the absolute value must be taken and it reflects the halfwidth of the peak, so $2 \Delta \theta$ is the peak of the peak (really half-width at half-height) so $2 \Delta \theta$ is the peak full-width at half-height, $\beta . \Delta d$ reflects the crystallite thickness,

Thickness $=t=\Delta d=\frac{\lambda}{\beta \cos \theta_{\beta}}$

If a Gaussian function is used to describe the peak a pre-factor of 0.9 occurs so the Scherer equation is given as $\mathrm{t}=0.9 \lambda / \beta \cos \theta_{\beta}$. The shape factor provides information about the "roundness" of the particle. For a spherical particle the shape factor is 1 , for all other particles it is smaller than 1 . The formula for this calculation is:

shape factor $=4 . \pi \cdot \frac{\text { area }}{\text { perimeter }}{ }^{2}$

Eq. (2.14)

The crystallographic phase identification was made possible by using X'pert Highscore software, where a match of the experimental diffraction data with the ICDD (International Council for Diffraction Data) database is made. 


\subsection{Color Measurements}

\subsubsection{CIE XYZ Color Space}

The three dimensional color space CIE XYZ (CIE 1931 Color Space) is a scientific system used to define light colors additive colors; first established by the International Commission on Illumination or CIE, which is the abbreviation for its French name, Commission international de l'éclairage. In the subsequent chapter on FWA (chapter 5), this color space would be commonly used to investigate the varying degrees of photoyellowing.

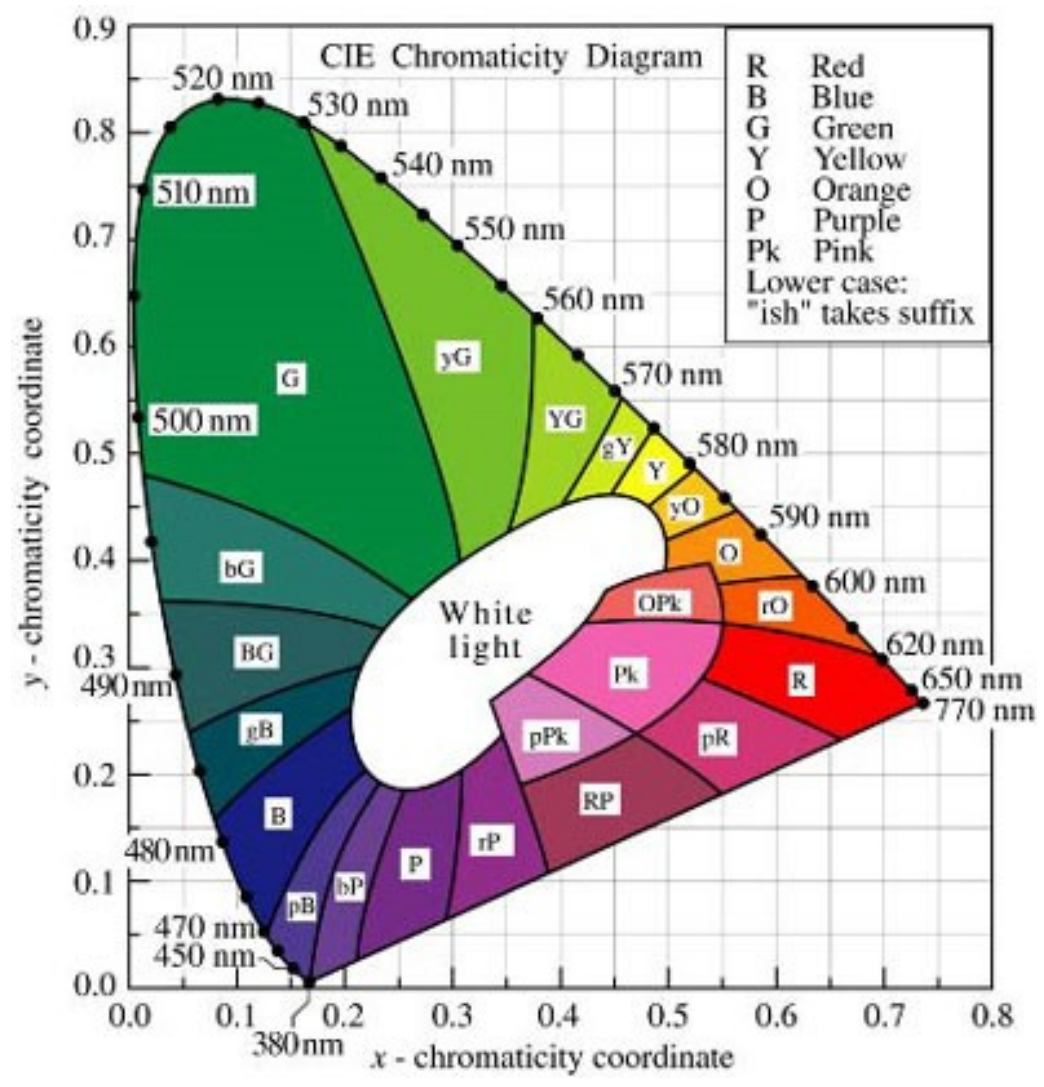

Figure 2.11: The CIE Gamut or Chromaticity chart 
The CIE used the data produced by Wright and Gold ${ }^{7}$ to develop the CIE color space and is now a widely accepted international standard for defining colors. CIE XYZ values or the Tristimulus values (The human eye has three different types of color sensitive receptors, called cone cells which have the ability to receive three different wavelengths of light, hence the need for three parameters to describe a color) is the measure of cone cell activation of three colour sensing cells in the retina of our eye, and are defined between $0 \%$ and $100 \% .{ }^{7}$ The CIE XYZ color spaces uses the values $\mathrm{X}, \mathrm{Y}$ and $\mathrm{Z}$ and were designed so that the value of $\mathrm{Y}$ is a measurement of the brightness of a color. The values of $\mathrm{X}, \mathrm{Y}$ and $\mathrm{Z}$ are used to derive the parameters of $\mathrm{x}$ and $\mathrm{y}$ to be able to plot the colors on the CIE gamut or chromaticity chart shown in figure 2.11.

The chromaticity chart is a two dimensional representation of the three dimensional CIE XYZ color space and displays all the chromaticity's which a person sees. Chromaticity in turn could be defined as the aspect of color which is determined by its dominant wavelength and purity; which is simply the color that we see. When all such chromaticities are displayed, it is called the gamut of human vision and when plotted in two dimensions forms an ellipsoid. ${ }^{8}$

All data were recorded using D65 2 Deg (Illu/Obs) [standard illuminant]. Colour measurements before and after UV irradiation were taken using Minolta Chroma Mater CR210, with a wide are illumination, a $2^{\circ}$ viewing angle and a $50 \mathrm{~mm}$ diameter measuring area to average the reading over a wide area, which is suitable for measuring cloth or textured surfaces. Each color measurement represents the average of nine readings. 


\subsection{Practical Considerations 2.4.1 Optical and Vibrational Spectroscopy}

UV-Visible Spectroscopy: Diluted solutions of the nanomaterial samples in suitable organic solvents were used for UV-VIS absorption measurement. The instrument used was a CARY 100Scan UV-Visible spectrophotometer operating in a double beam mode. The measurements were carried out with a data interval of $1 \mathrm{~nm}$ and a scan rate of 200 $\mathrm{nm}$ per minutes. The measured range spanned from $300-800 \mathrm{~nm}$.

Photoluminescence Spectroscopy: One of the primary ways to study and characterize luminescent nanoparticles is by the use of spectroscopic techniques. Semiconductors are distinguished by the nature and the mechanisms of the processes that bring out the absorption or emission of light. Photoluminescence excitation has become a standard technique for obtaining information on nanostructured materials such as quantum dots. In bulk materials, the luminescence spectrum often resembles a standard direct absorption spectrum, thus making it of little use to study details of both. Since the discovery of room-temperature photo-luminescence from porous silicon, considerable effort has been devoted to the development of silicon nanostructure-based light emission sources. The luminescence of Si nanocrystals has been studied in various systems, from single nanocrystals to multilayered structures, and efficient electroluminescence has also been reported. 
The origin of photoluminescence from silicon nanocrystals has been attributed to excitonic emission in a quantum-confined system. ${ }^{8}$ when an exciton is created in a nanocrystal with dimensions smaller than the excitonic Bohr radius, the electrons and holes are confined in real and reciprocal space. The Bohr radius of bulk silicon is $4.9 \mathrm{~nm}$ and is characteristic of each material. ${ }^{9}$ By disrupting the crystal symmetry, quantum confinement causes a relaxation of the momentum selection rules in the silicon nanocrystal. Radiative recombination is therefore a much more efficient process in silicon nanocrystals than in bulk silicon, and the emission efficiency shoots up accordingly. ${ }^{10}$ Quantum-confined nanocrystals, or quantum dots, based on a wide range of semiconductor materials, are now being widely investigated as emitters, detectors, biological sensors, and solar cells.

The photoluminescence technique involves scanning the frequency of the excitation signal and recording the emission within a very narrow spectral range. The excitation source is a Xenon flash tube, which produces an intense, short duration pulse of radiation over the spectral range of the instrument. Energy from the source is focussed by an ellipsoidal mirror and reflected by a torroidal mirror onto the entrance slit of an Excitation monochromator. The monochromator consists of an entrance slit, a grating, a spherical mirror and an exit slit. A narrow wavelength band emerges from the exit slit, with the centre wavelength being determined by the setting of the grating. The majority of the excitation beam is however transmitted to the sample area with the aid of a focussing torroidal mirror. A small portion is reflected by the beam splitter onto a reference photodiode. Energy emitted from the sample is then focussed by the torroidal 
mirror onto the entrance slit of the emission monochromator, which consists of an entrance slit, a grating and an exit slit. A narrow wavelength band emerges from the exit slit with the centre wavelength again being determined by the setting of the grating. The excitation and emission monochromator can be scanned over their ranges independently to selected points in their ranges.

Photoluminescence measurements were conducted using a J-Y Fluorolog fluorescence meter. The samples were dissolved in suitable organic solvents and the photoluminescence measured over a range of 200-800 $\mathrm{nm}$.

\subsubsection{Vibrational Spectroscopy- Fourier Transform Infrared Spectroscopy (FTIR)}

Vibrational Spectroscopy involves photons that induce transitions between vibrational states in molecules and solids, typically in the infrared frequency range from 2 to 12 $\times 10^{13} \mathrm{~Hz}$. The energy gaps of many semiconductors are in this same frequency range and can be studied by infrared techniques. FTIR spectroscopy is crucial in studying the surface properties of capped semiconductor particles viz silicon and germanium nanocrystal whose surface has been modified by hydrophobic and hydrophilic surface molecules.

A Bruker Tensor 27 infra-red spectrometer was used in this research. The samples were prepared using the $\mathrm{KBr}$ pellet technique. 


\subsubsection{BET surface area (Brunauer, Emmett, and Teller)}

BET surface area measurements were conducted using a Micromeritics ASAP 2010 Accelerated Surface Area and Porosimetry System. The samples were initially dried in vacuum, followed by degassing at $100^{\circ} \mathrm{C}$ under $\mathrm{N}_{2}$ prior to measurements. The BET method according to ASTM D 45567-86 Standard Test Method; for Single Point Determination of Specific Area of Catalysts, Using Nitrogen Adsorption by the Continuous Flow Method. 


\subsubsection{High performance Liquid Chromatography (HPLC)}

\section{Measurements}

HPLC assays for the determination of the production of reactive oxygen species (ROS) by fluorescent whitening agents were performed. A Dionex Ultimate 3000 system equipped with a Bio-Rad Hi-Pore reversed phase column (RP-304), $250 \times 4.6 \mathrm{~mm}$, along with a guard, was used for this assay. An isocratic mobile phase containing acetonitirile and a $\mathrm{Na}_{2} \mathrm{HPO}_{4}$ buffer $\left(0.033 \mathrm{~mol}^{-1}, \mathrm{pH} 8\right)$ in the ratio of 7:15 was used at a flow rate of $0.5 \mathrm{ml} / \mathrm{min}$. The eluants were monitored at $351 \mathrm{~nm}$. The analysis was performed at $22^{\circ} \mathrm{C}$ and an equilibration time of 10 minutes was used in between runs. Solutions of $1 \mathrm{mM}$ LUM were analyzed before and after irradiation with or without the addition of $0.1 \mathrm{mM}$ APA, $0.2 \mathrm{mM}$ rose bengal, $2 \mathrm{mM}$ L-ascorbic acid, $0.25 \mathrm{mM}$ Uvitex, $0.3 \mu \mathrm{M}$ amine-capped silicon nanoparticles, $2 \mu \mathrm{M}$ alkene-capped silicon nanoparticles, $0.3 \mu \mathrm{M}$ amine-capped germanium nanoparticles and $2 \mu \mathrm{M}$ alkene-capped germanium nanoparticles. 


\subsection{References}

1. Cushing, B. L.; Kolesnichenko, V. L.; O'Connor, C. J., Recent advances in the liquid-phase synthesis of inorganic nanoparticles. Chem. Rev.2004, 104, 3893-3946.

2. Rao, C. N. R.; Muller, A.; Cheetham, A. K., The Chemistry of Nanomaterials: Synthesis, Properties and Applications.2004.

3. Schmid,G.; Ed. Nanoparticles From Theory to Application; WILEY-VCH Verlag, Weinheim, Germany, 2004.

4. Williams, D. B. and Carter, C. B. Transmission Electron Microscopy Basics 1, Plenum Press, New York, United States of America, 1996.

5. Billinge, S. J. L and Dinnebier, R. E. Powder diffraction: Theory and Practice; RSC publishing, 2008.

6. (a) Goldstein, D. Newbury, D. Joy, C, Lyman, P. Echlin, E. Lifshin, L. Sawyer, Scanning Electron Microscopy and X-Ray Microanalysis , Kluwer Academic, Plenum Publishers, New York; 2003. (b) http://www.siliconfareast.com/SEMTEM.htm

7. Hunt, R.W.G., Measuring Colour, Fountain Press, England, 1998

8. Wyszecki, G and Stiles, W.S.,Color Science, John Wiley \& Sons, New York 1982.

9. http://www.ou.edu/research/electron/bmz5364/resolutn.html 


\section{Solution Synthesis and Characterization of Silicon Nanocrystals}

\subsection{Introduction}

This chapter deals with the synthesis and characterization of crystalline, photoluminescent silicon nanocrystals using solution techniques. Silicon nanocrystals have attracted an increasing amount of attention over the last few years, as they combine sizedependent optical properties with the richness and stability of silicon surface chemistry. ${ }^{1}$ Since quantum confinement effects for Si nanocrystals are expected for particles smaller than $\sim 5 \mathrm{~nm}$ (Bohr radius of silicon= $4.9 \mathrm{~nm}$ ) the controlled synthesis of well-defined and monodisperse Si nanocrystals in the 1-5 nm range is of interest for a variety of optoelectronic applications. ${ }^{2-4}$

The synthesis of Si nanocrystals using hydrogen terminated Si surfaces which could react with terminal alkenes and amines to provide $\mathrm{Si}-\mathrm{C}$ and $\mathrm{Si}-\mathrm{N}$ linkages is an attractive way of synthesizing functionalized Si nanocrystals. ${ }^{5,6}$ Quite a few routes for such functionalized nanocrystals have been previously reported. Some of the methods used to functionalize the surface of the silicon nanocrystals are similar to those used for porous or planar silicon surfaces. ${ }^{7,8}$ The synthesis of H-terminated Si nanocrystals have been made possible by methods such as annealing $\mathrm{SiO}_{2}$ at high temperatures followed by etching with $\mathrm{HF}$ or plasma synthesis or the reduction of $\mathrm{SiCl}_{4}$ with alkali naphthalide or Zintl salts or the metathesis reaction between sodium silicide and $\mathrm{NH}_{4} \mathrm{Br}^{9-16,24}$ However, the method yielding functionalized Si nanocrystals, that are both hydrophobic and 
hydrophilic are that of Tilley et al who synthesized alkyl and amine terminated $\mathrm{Si}$ nanocrystals in inverse micelles using hydride reducing agents. ${ }^{5,17}$

In the literature there are very few reports on the size-controlled synthesis of silicon nanocrystals. The primary aim of this chapter is to illustrate the possibility of size control by varying reactions conditions. Experiments 1 to 3 deal with the synthesis of silicon nanocrystals using three different surfactants. The effect of surfactant on particle size is discussed. Experiments 4 to 6 discuss the effect of various hydride reducing agents on particle size, whilst Experiments 7 and 8 shows the control of particle size by varying precursors. Finally, experiments 9 to 11 discuss the optical properties of $\mathrm{Si}$ nanocrystals whilst using four different capping agents. The characterization of these nanocrystals by TEM, PL and IR spectroscopy is reported. The various reaction parameters used in the synthesis are tabulated in tables 3.1-3.3. 


\subsection{The Synthesis of Alkyl- and Amine-Functionalized}

\section{Silicon Nanocrystals}

Alkyl- and amine-capped Si nanocrystals were synthesized according to the following procedure. Dry toluene or hexane was made oxygen free by a cycle of freeze-pumpthaw cycles and kept under $\mathrm{N}_{2}$ throughout the synthesis. The surfactants TOAB (Tetraoctyl ammonium bromide- $\left.\mathrm{CH}_{3}\left(\mathrm{CH}_{2}\right)_{7}\right]_{4} \mathrm{NBr}$ ) or $\mathrm{C}_{12} \mathrm{E}_{5}$ (Pentaethylene glycol monododecyl ether-H- $\left.\left(\mathrm{OCH}_{2} \mathrm{H}_{2}\right)_{5} \mathrm{OCH}_{2}\left(\mathrm{CH}_{2}\right)_{10} \mathrm{CH}_{3}\right)$ or CTAB (Cetyl trimethylammonium bromide- $\left.\left(\mathrm{C}_{16} \mathrm{H}_{33}\right) \mathrm{N}\left(\mathrm{CH}_{3}\right)_{3} \mathrm{Br}\right)$ were dispersed in the solvents and the precursor's silicon tetrachloride $\left(\mathrm{SiCl}_{4}\right)$, silicon tetrabromide $\left(\mathrm{SiBr}_{4}\right)$ or silicon tetraiodide $\left(\mathrm{SiI}_{4}\right)$ were either injected or dissolved (in the case of $\mathrm{SiI}_{4}$, an off-white powder) into the solvents using air tight syringes. After the mixture was homogenized, hydride reducing agents $\left(\mathrm{LiAlH}_{4}\right.$ or $\mathrm{Li}\left(\mathrm{Et}_{3}\right) \mathrm{BH}$ or $\mathrm{LiBH}_{4}$ or $\left.\mathrm{NaBH}_{4}\right)$ was added to form hydrogen terminated Si nanocrystals. After the mixture was allowed to react for $30 \mathrm{mins}$, the surface of the hydrogen terminated nanocrystals were modified using capping agents (1-Hexene, 1Heptene or 1-Octene or allylamine) with the addition of a catalytic amount of $\mathrm{H}_{2} \mathrm{PtCl}_{6}$ solution.

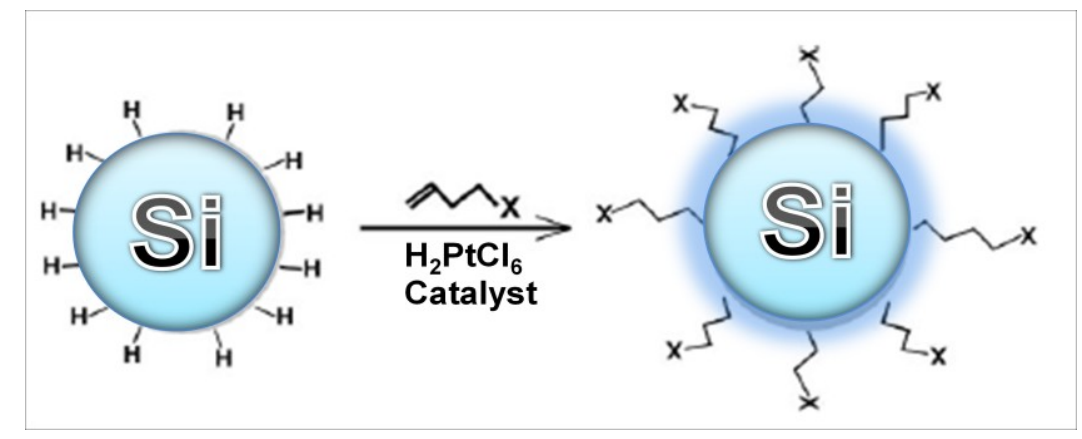

Figure 3.1: A schematic representation of the microemlusion synthesis. 
The variables in the synthesis such as surfactants, precursors, reducing agents and capping agents were then used to study their effect on particle size and optical properties.

\subsubsection{Effect of Surfactants on Particle Size (Expt. 1-3)}

\begin{tabular}{|l|l|l|l|l|}
\hline E.No & Surfactant & Solvent & Reductant & Capping agent \\
\hline 1 & $\begin{array}{l}\text { TOAB (Tetraoctyl ammonium } \\
\text { bromide) }\end{array}$ & Toluene & $\mathrm{LiAlH}_{4}$ & 1 -Hexene \\
\hline 2 & $\begin{array}{l}\mathrm{C}_{12} \mathrm{E}_{5} \quad(\text { Pentaethylene glycol } \\
\text { monododecyl ether) }\end{array}$ & $\begin{array}{l}\text { Hexane } \\
\text { nium bromide) }\end{array}$ & $\mathrm{LiAlH}_{4}$ & $1-$ Hexene \\
\hline 3 & Thetyl trimethylammo- & Toluene & $\mathrm{LiAlH}_{4}$ & $1-$ Hexene \\
\hline
\end{tabular}

Table 3.1: List of surfactants used to synthesize Si nanocrystals

Crystalline Si nanocrystals of sizes ranging from 1.5-6 nm were synthesized while studying the effect of surfactants, on the particle size of silicon nanocrystals. All other variables (Table 3.1) were kept constant. 


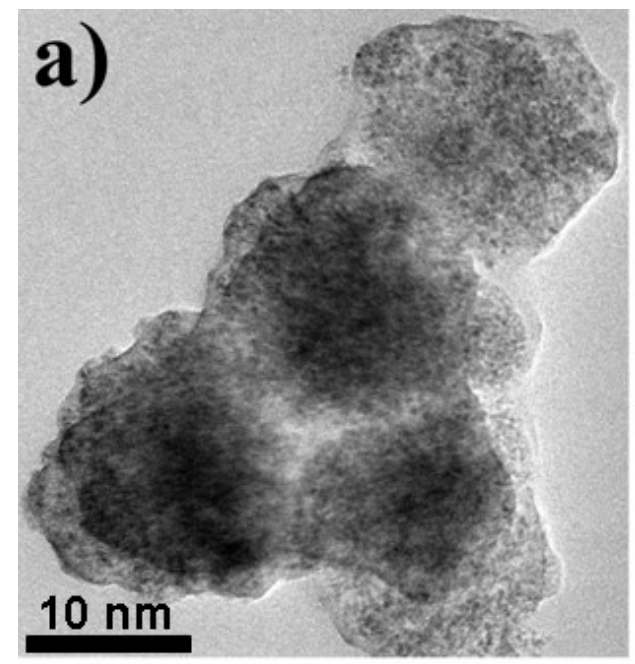

\section{b)}
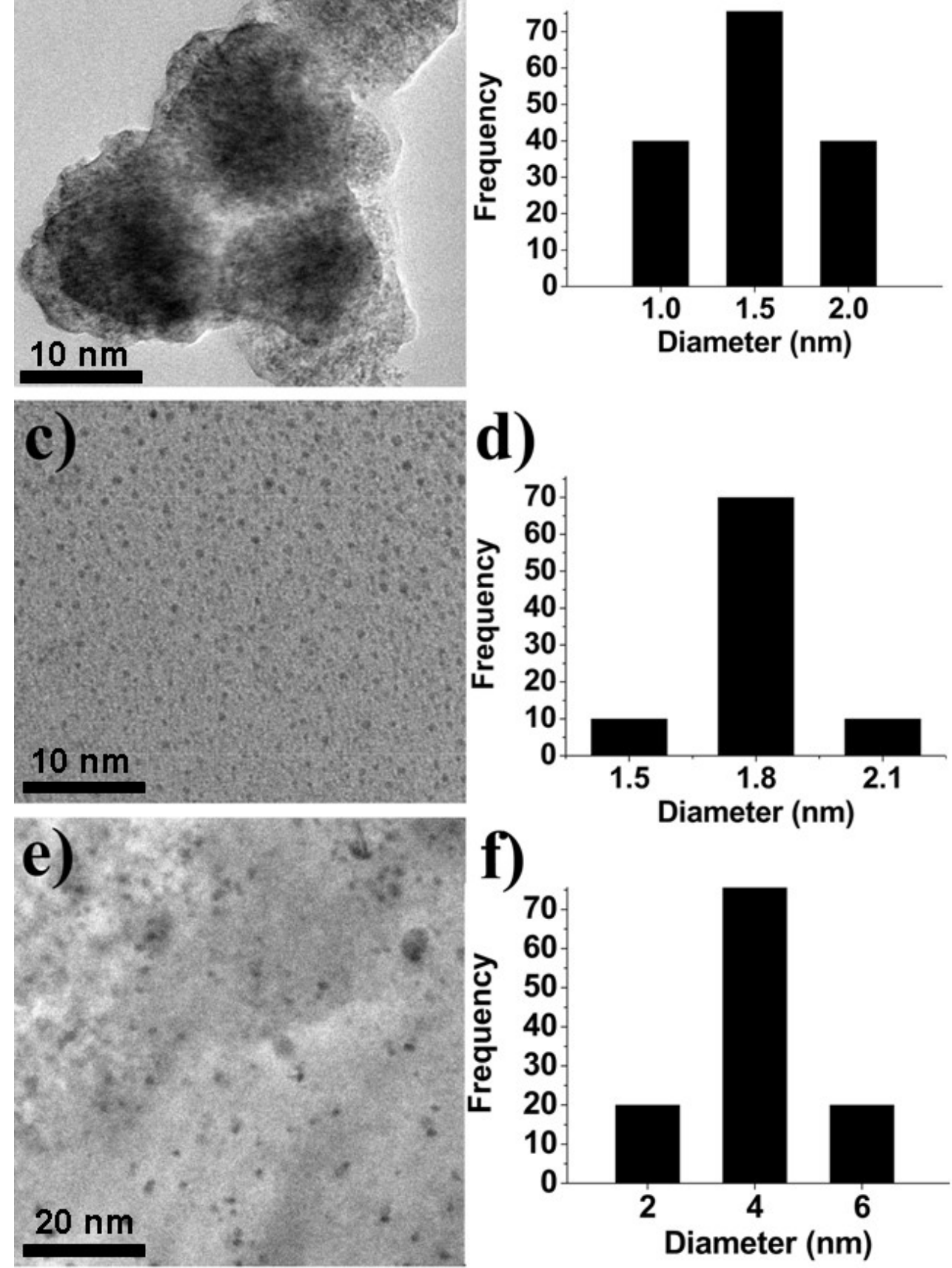

Figure 3.2: Low magnification TEM images of silicon nanocrystals obtained using the surfactants (a) TOAB, (c) $\mathrm{C}_{12} \mathrm{E}_{5}$ and (e) $\mathrm{CTAB}$ and their corresponding size distribution histograms (b), (d) and (f) respectively. 
Experiment 1 (TOAB) produced Si nanocrystals of size $1.5 \pm 0.5 \mathrm{~nm}$ (based on 250 particles) and were crystalline and spherical. Figure 3.2a is a typical low magnification TEM image of the reaction using TOAB as surfactant. Although particles of narrow size distribution were observed (Figure 3.2b), the particles were aggregated into clusters of 50-80 nm with each cluster consisting of a few hundred nanocrystals. Further the nanocrystals appeared to be encapsulated in an organic matrix. EDX analysis of the nanocrystals in Figure 3.2a shows the presence of a Br peak (Figure 3.3) indicating that remnants of the surfactant were still present in the nanocrystals. Repeated purification of the nanocrystals by liquid phase separation failed to completely remove residual surfactant and other organic impurities that tend to encapsulate these particles.

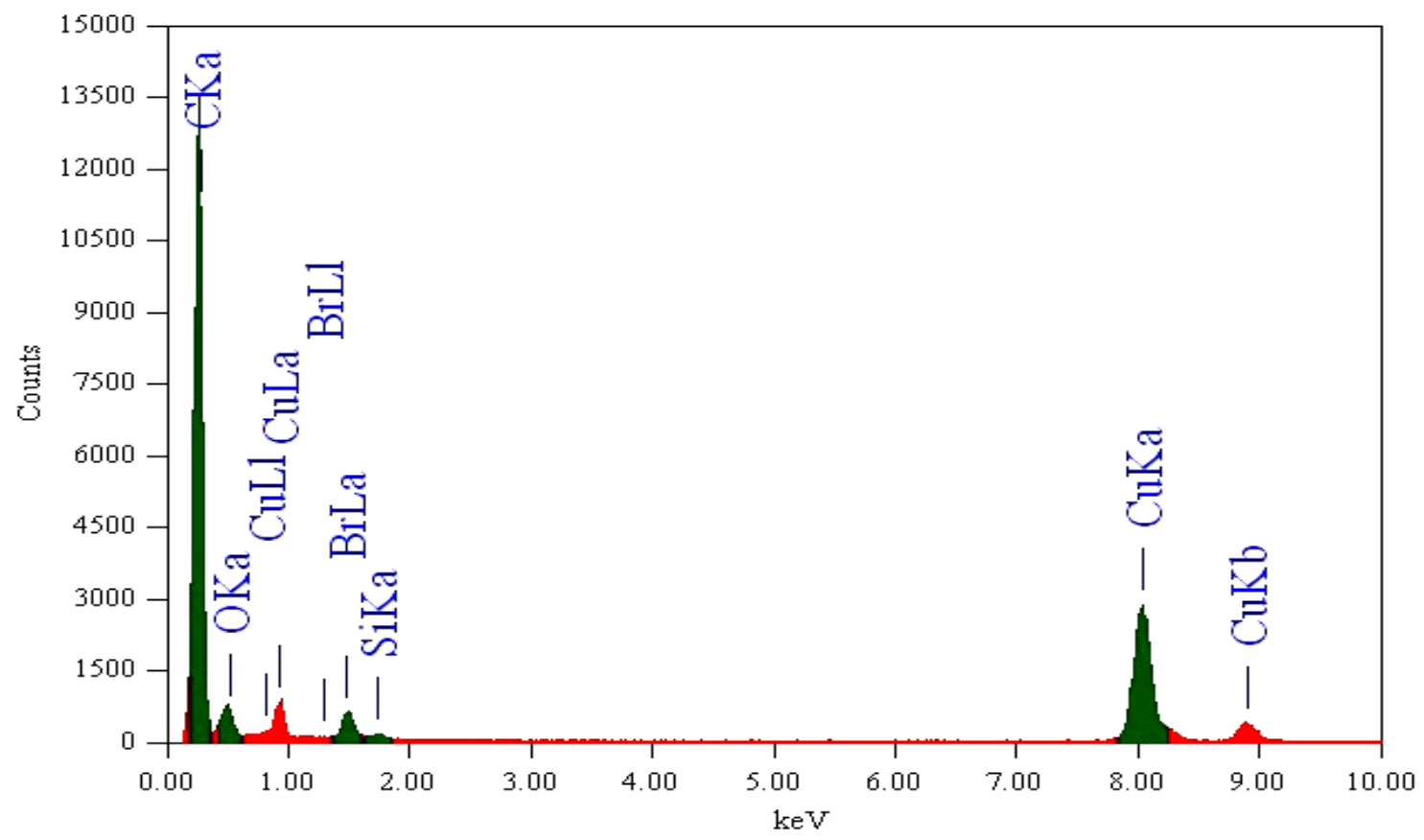

Figure 3.3: EDX spectrum of a cluster of Si nanocrystals synthesized using TOAB as surfactant 
Figure 3.4 is a SAED pattern of the area shown in figure $3.2 \mathrm{a}$, with the rings corresponding to the diamond crystal structure adopted by silicon. The weak rings are due to the organic surfactant layer on the nanocrystals.

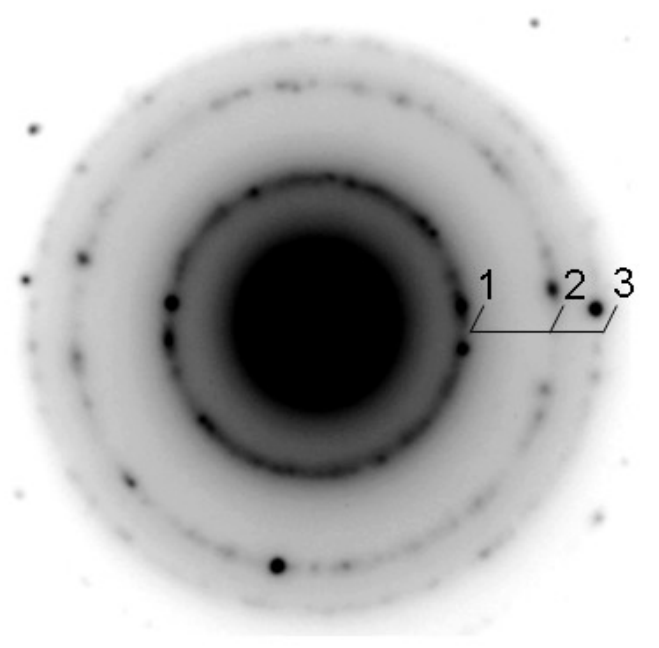

Figure 3.4: Selected-area electron diffraction (SAED) for silicon nanocrystals obtained from the reaction using TOAB as surfactant.

The reaction using $\mathrm{C}_{12} \mathrm{E}_{5}$ as surfactant (Experiment 2) also produced crystalline and spherical nanocrystals as can be seen in figure 3.2c. Si nanocrystals of average size 1.8 $\mathrm{nm}$ with a range of sizes of $\pm 0.3 \mathrm{~nm}$ were synthesized. The particles were of narrow size distribution as shown in the histogram (figure 3.2d) and were found to be free standing nanocrystals. EDX analysis (figure 3.5) of the sample showed the presence of silicon and oxygen, the latter probably due to some surface oxidation. SAED pattern of the nanocrystals showed the characteristic diamond crystal structure of lattice and was similar to that obtained for the previous experiment. Compared to the previous reaction involving TOAB as surfactant, this reaction $\left(\mathrm{C}_{12} \mathrm{E}_{5}\right)$ was relatively easier to purify. This is mainly because whilst using TOAB, a white powder that is sparingly soluble in polar 
solvents is obtained. $\mathrm{C}_{12} \mathrm{E}_{5}$ is a viscous liquid ( $\rho=.963 \mathrm{~g} / \mathrm{ml}$ ), and is easily removed by repeated washings with polar solvents due to its better solubility in polar solvents compared to TOAB.

The reaction using CTAB (Experiment 3) as surfactant produced nanocrystals of average size $4.0 \mathrm{~nm}$ with a size range of $\pm 2 \mathrm{~nm}$ (Figures $3.2 \mathrm{e}$ and f). Figure $3.2 \mathrm{e}$ is a typical low magnification image showing nanocrystals encrusted in surfactant. Although CTAB has relatively higher solubility compared to the former surfactants, an inability to completely remove residual surfactants was faced. Though SAED analysis of the particles showed that they were crystalline silicon (Figure 3.3), EDX analysis showed the presence of a Br peak, due to the remnants of surfactant, similar to that observed for TOAB.

The ease of purification and relative narrow size distribution obtained for the reaction involving the surfactant $\mathrm{C}_{12} \mathrm{E}_{5}$ prompted its use for further studies in this chapter. 


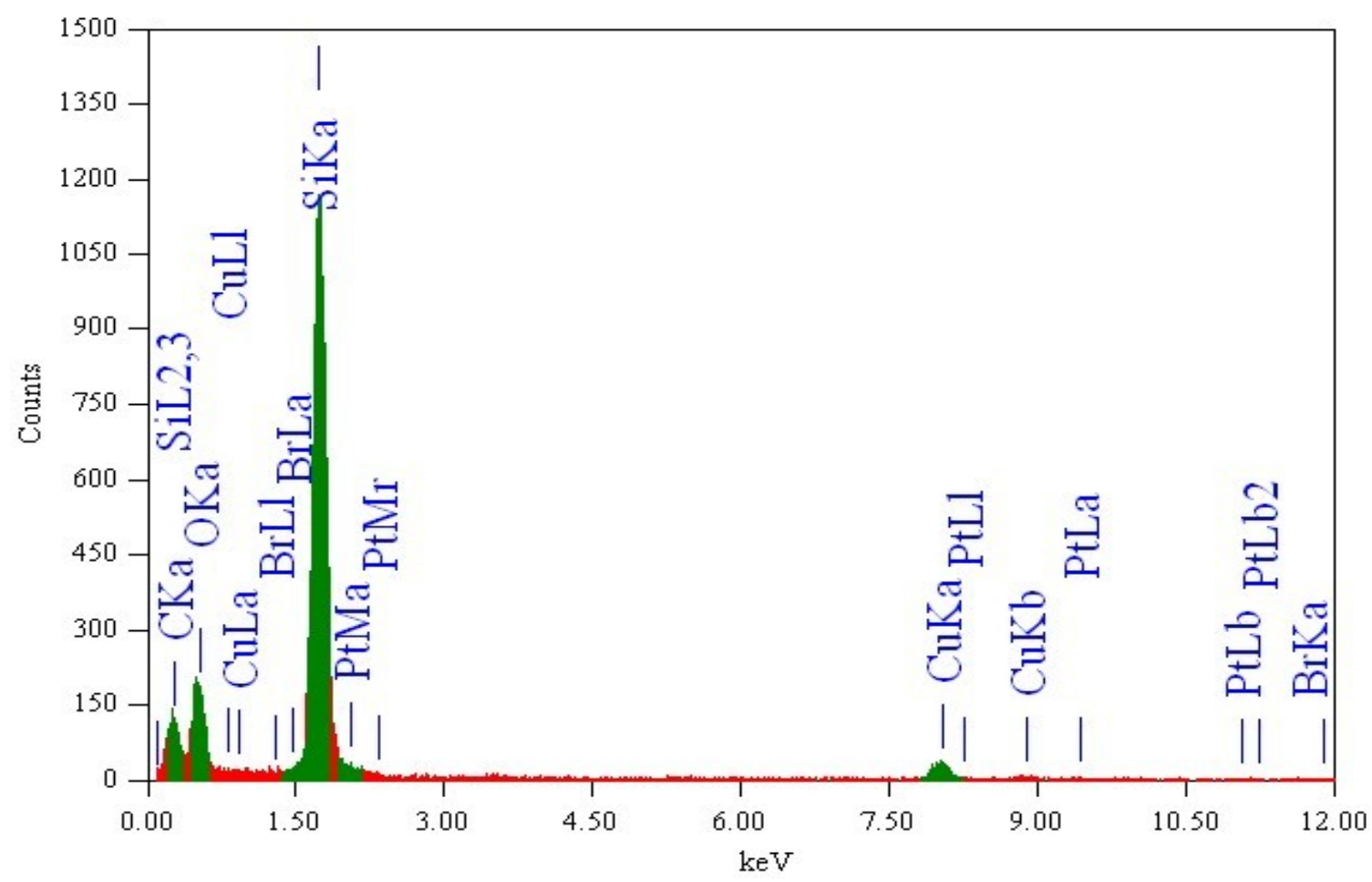

Figure 3.5: EDX spectrum of silicon nanocrystals obtained using $\mathrm{C}_{12} \mathrm{E}_{5}$ as surfactant 


\subsubsection{Effect of Reducing agents on Particle size (Expt. 2, 4-6)}

The effects of different hydride reducing agents on the particle size of Si nanocrystals were investigated. All other variable were kept constant (Table 3.2). Si nanocrystals of size range 1.8 to $2.5 \mathrm{~nm}$ were synthesized.

\begin{tabular}{|l|l|l|l|l|}
\hline E.No & Reductant & Surfactant & Solvent & Capping agent \\
\hline 2 & $\mathrm{LiAlH}_{4}$ & $\mathrm{C}_{12} \mathrm{E}_{5}$ & Hexane & 1 -Hexene \\
\hline 4 & $\mathrm{Li}(\mathrm{Et})_{3} \mathrm{BH}$ & $\mathrm{C}_{12} \mathrm{E}_{5}$ & Hexane & 1 -Hexene \\
\hline 5 & $\mathrm{LiBH}_{4}$ & $\mathrm{C}_{12} \mathrm{E}_{5}$ & Hexane & $1-$ Hexene \\
\hline 6 & $\mathrm{NaBH}_{4}$ & $\mathrm{C}_{12} \mathrm{E}_{5}$ & Hexane & $1-$ Hexene \\
\hline
\end{tabular}

Table 3.2: List of hydride reducing agents used to synthesize Si nanocrystals

Silicon nanocrystals of size Si nanocrystals of average size $1.8 \mathrm{~nm}$ with a range of sizes of $\pm 0.3 \mathrm{~nm}$ were synthesized (Experiment 2) whilst using lithium aluminium hydride as reducing agent as seen earlier in figure $3.2 \mathrm{c}$ and d. Similarly, $2 \mathrm{~nm}$ particles of size range $\pm 0.2 \mathrm{~nm}$ and $2 \mathrm{~nm}$ particles of size range $\pm 0.5 \mathrm{~nm}$ were obtained for the reduction using lithium tri-ethyl borohydride and lithium borohydride as reductant (Figures 3.6a to 3.6 d). However the reaction with sodium borohydride produced silicon particles of average size $5 \mathrm{~nm}$ with size range $\pm 1 \mathrm{~nm}$ (Figures $3.6 \mathrm{e}$ and $\mathrm{f}$ ).

The vigorous and quick reduction caused by the reductant $\mathrm{LiAlH}_{4}$ causes a fast depletion of monomers with few left for any further growth of the silicon nanocrystals, resulting 
in the formation of smaller nanocrystals. Lithium tri-ethyl borohydride and lithium borohydride on the other hand have comparatively weaker reactivity than lithium aluminum hydride, hence slightly larger particle sizes are observed. Thus, sodium borohydride which is known to have a weaker reducing ability produced particles of comparatively larger size distributions. When there is lesser monomer depletion in the nucleation burst, considerable amount of un-reacted monomers will contribute to the nanocrystal growth causing the formation of nanoparticles of large size.

The as-synthesized nanocrystals, after purification was found to be crystalline and their diffraction patterns corresponded to the characteristic diamond crystal structure adopted by silicon. 


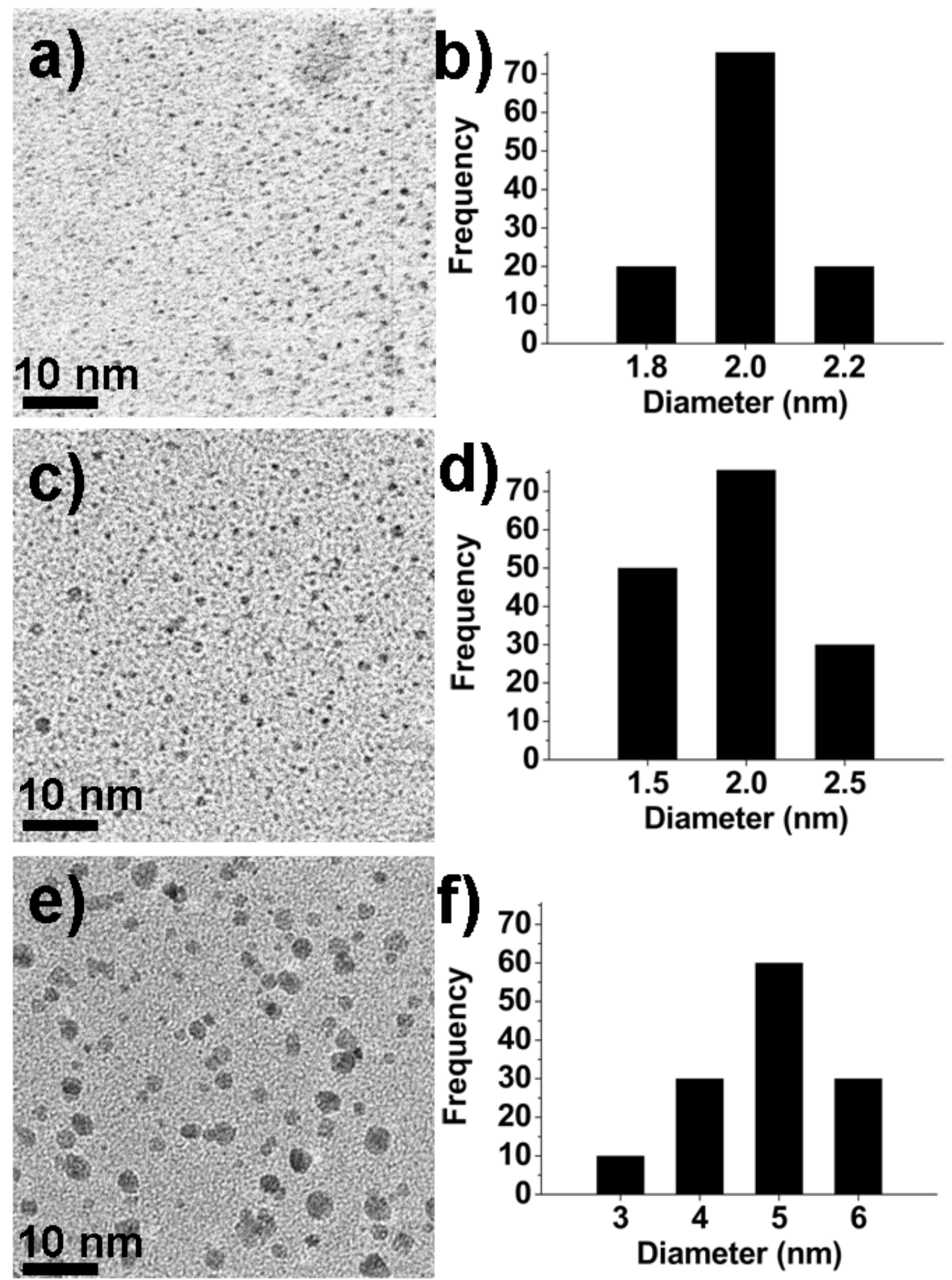

Figure 3.6: Low magnification TEM images of silicon nanocrystals obtained using the reductants (a) $\mathrm{Li}(\mathrm{Et})_{3} \mathrm{BH}$, (c) $\mathrm{LiBH}_{4}$ and (e) $\mathrm{NaBH}_{4} \mathrm{CTAB}$ and their corresponding size distribution histograms (b), (d) and (f) respectively. 


\subsubsection{Effect of Precursors on Particle Size (Expt. 2, 7 and 8)}

Three different silicon halide precursors were used to synthesize Si nanocrystals. Both silicon tetrachloride and silicon tetrabromide were used as obtained, however silicon tetraiodide being a solid was first dissolved in toluene in a glove-box filled with $\mathrm{N}_{2}$ and transferred to an air-tight syringe.

\begin{tabular}{|c|c|c|c|c|}
\hline E.No & Precursor & Surfactant & Reductant & Capping agent \\
\hline 2 & $\mathrm{SiCl}_{4}$ & $\begin{array}{l}\mathrm{C}_{12} \mathrm{E}_{5} \text { (Pentaethylene glycol } \\
\text { monododecyl ether) }\end{array}$ & $\mathrm{LiAlH}_{4}$ & 1-Hexene \\
\hline 7 & $\mathrm{SiBr}_{4}$ & $\begin{array}{l}\mathrm{C}_{12} \mathrm{E}_{5} \text { (Pentaethylene glycol } \\
\text { monododecyl ether) }\end{array}$ & $\mathrm{LiAlH}_{4}$ & 1-Hexene \\
\hline 8 & $\mathrm{SiI}_{4}$ & $\begin{array}{l}\mathrm{C}_{12} \mathrm{E}_{5} \text { (Pentaethylene glycol } \\
\text { monododecyl ether) }\end{array}$ & $\mathrm{LiAlH}_{4}$ & 1-Hexene \\
\hline
\end{tabular}

Table 3.3: List of silicon halide precursors used to synthesize Si nanocrystals

As discussed in section 3.2.1 (experiment 2), the reaction using silicon tetrachloride $\left(\mathrm{SiCl}_{4}\right)$ as precursor yielded particles of size range $1.8 \pm 0.3 \mathrm{~nm}$ (Figure 3.2a and $\left.\mathrm{b}\right)$. The reaction using silicon tetrabromide $\left(\mathrm{SiBr}_{4}\right)$ as precursor produced nanocrystals of size $4.5 \pm 0.5 \mathrm{~nm}$ as can be seen in the histogram in the inset of figure 3.6a, and were highly crystalline. Figure 3.7a is typical low magnification TEM image of silicon nanocrystals synthesized using silicon tetrabromide as precursor. A selected area electron diffraction analysis (SAED) pattern of the particles shown in figure $3.7 \mathrm{~b}$, corresponded to the diamond crystal structure of silicon. Silicon tetrabromide has weaker bond strength 


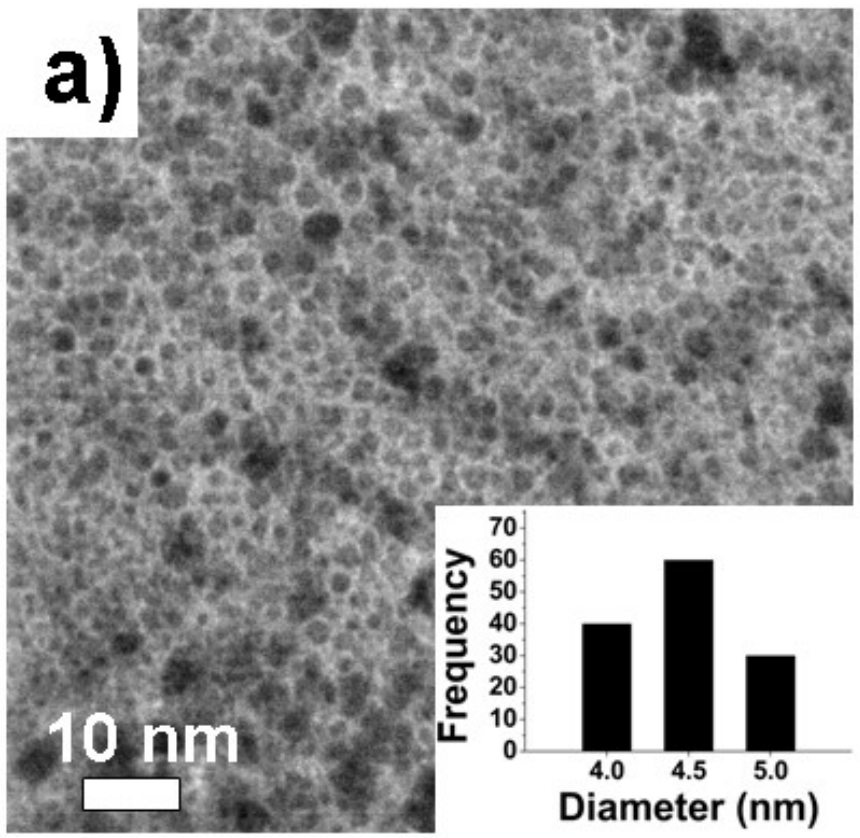

\section{b)}

Figure 3.7(a): Low magnification TEM image of silicon nanocrystals obtained using the precursor $\mathrm{SiBr}_{4}$ and (b) corresponding SAED pattern 
compared to silicon tetrachloride and is expected to produce smaller particles; on the contrary bigger particle sizes are obtained. This is possibly due to the varied reactivity of the $\mathrm{Br}^{-}$ions in the solvent medium compared to that of $\mathrm{Cl}^{-}$. Hence the rate at which silicon tetrabromide is reduced is much slower compare to silicon tetrachloride, thus forming bigger nanocrystals.

The reaction of silicon tetraiodide with lithium aluminium hydride failed to produce crystalline Si particles. On injection of the reductant $\left(\mathrm{LiAlH}_{4}\right)$ into the precursor solution, no immediate colour change was observed. The liberation of white solids of lithium iodide or aluminium iodide was observed. The absence of any colour in the reaction mixture could be either because of the absence of any reaction between $\mathrm{SiI}_{4}$ and $\mathrm{LiAlH}_{4}$, or due to the oxygen free nature of the experiment; since both aluminium iodide and lithium iodide turn yellow and brown in colour when exposed to oxygen. 


\subsection{Optical Properties of Silicon Nanocrystals}

\subsubsection{Effect of Particle Size on the Optical Properties}

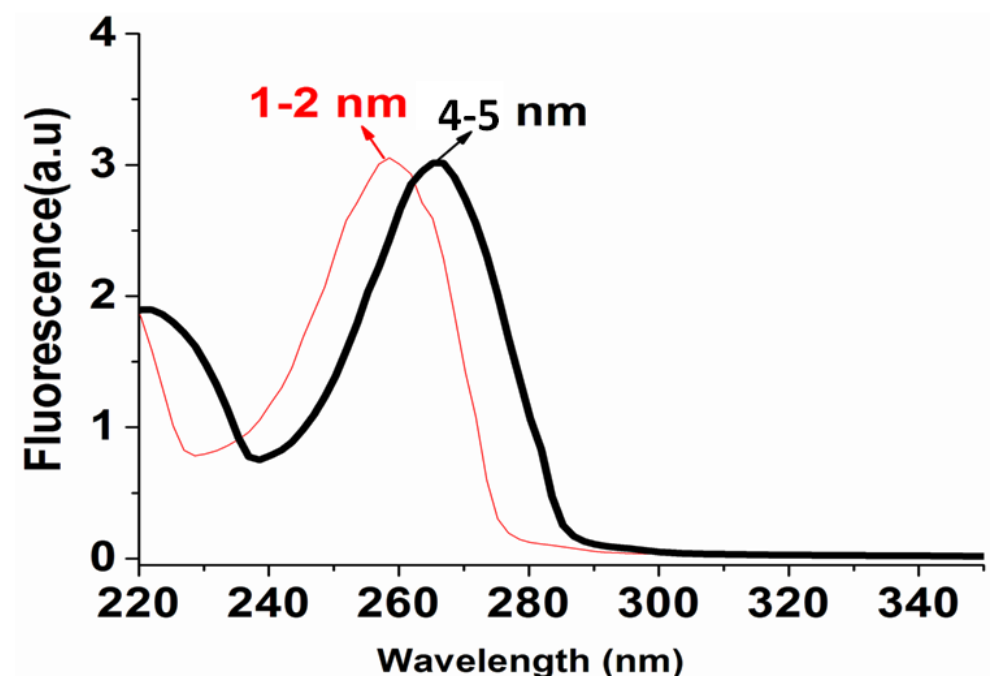

Figure 3.8: (a) Absorption spectra of 1-Hexene capped (a) 1-2nm and (b) 4-5 nm silicon nanocrystals

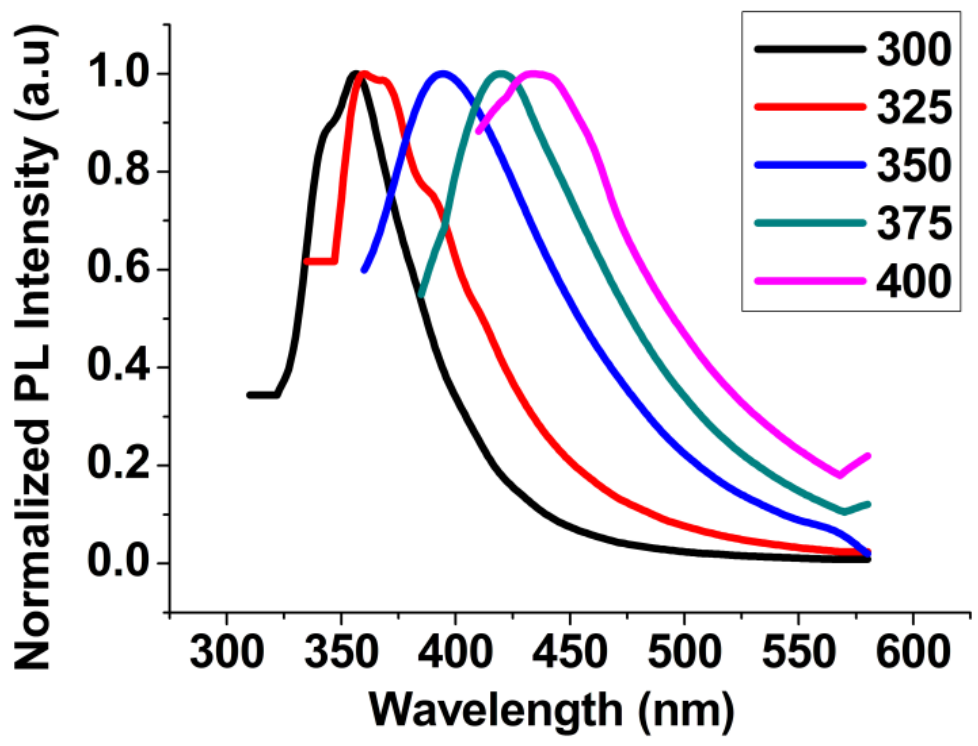

Figure 3.9: Photoluminescence spectra of 1-2 nm silicon nanocrystals 
The UV/Vis absorption spectra (Figure 3.8) of 1-Hexene capped silicon nanocrystals of size 1-2 $\mathrm{nm}$ (a) and 4-5 $\mathrm{nm}$ (b) display optical transitions in the 230- $280 \mathrm{~nm}$ range. Figure 3.9 shows the normalized photoluminescence spectra of 1-2 nm silicon nanocrystals, exhibiting peaks that range from $340 \mathrm{~nm}$ (for the $300 \mathrm{~nm}$ excitation) to $450 \mathrm{~nm}$ (for the $400 \mathrm{~nm}$ excitation). The photoluminescence peak position appears to be red shifted as the excitation wavelength is incremented form $200 \mathrm{~nm}$ to $400 \mathrm{~nm}$. A similar trend is observed for the photoluminescence spectra of 4-5 nm silicon nanocrystals shown in figure 3.10. The 4-5 nm silicon nanocrystals exhibit photoluminescence in the region of $250 \mathrm{~nm}$ to $480 \mathrm{~nm}$.

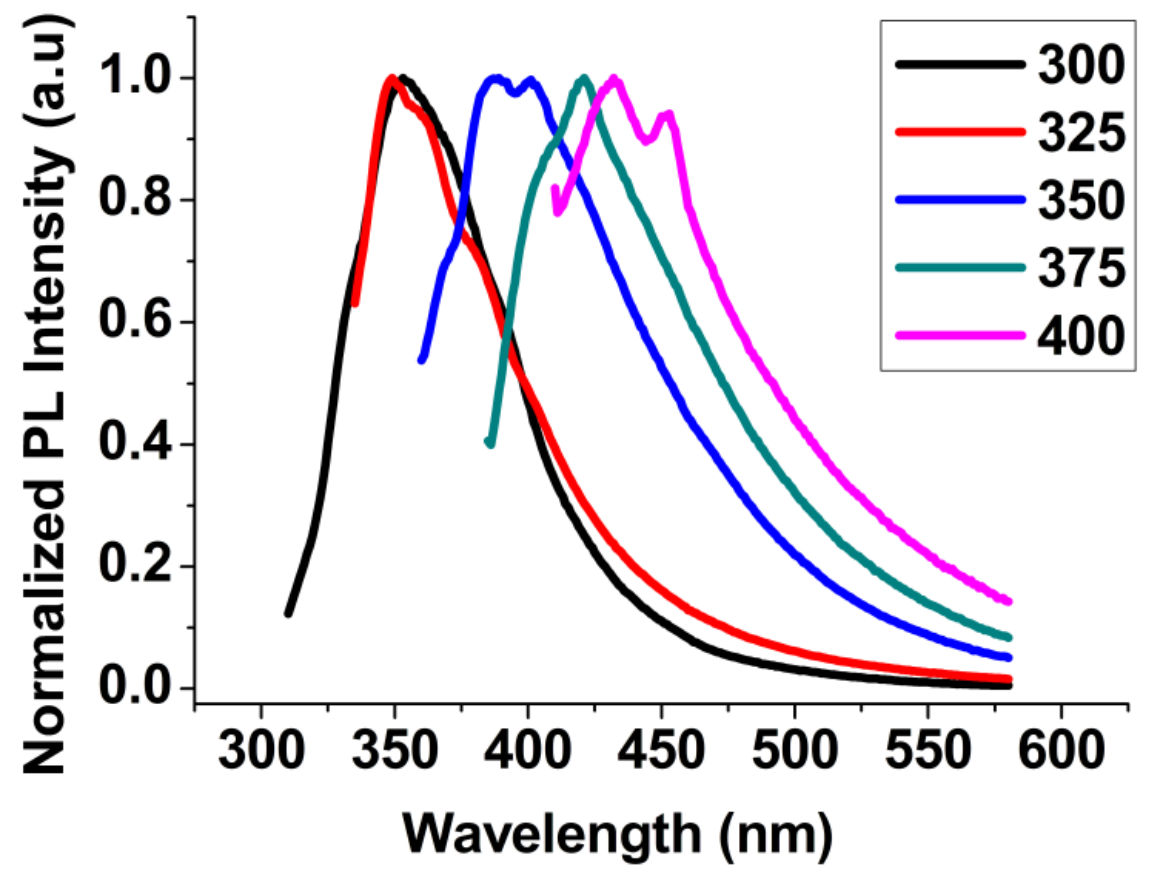

Figure 3.10: Photoluminescence spectra of $4-5 \mathrm{~nm}$ silicon nanocrystals 
Quantum confinement effects result in optical properties that are highly size-dependent, e.g., smaller nanocrystals absorb and emit light at higher energies than larger nanocrystals. Whilst bulk Si is, due to its indirect band-gap electronic structure, a very inefficient emitter, even at liquid He temperatures; Si nanocrystals absorb strongly in the visible and ultraviolet region. The narrow emission that is observed for both 1-2 nm and 4-5 nm nanocrystals in figures 3.9 and 3.10 are consistent with those reported in the literature and reflects on the monodispersity of the nanocrystals. ${ }^{18}$ A monotonic shift in the photoluminescence as the excitation wavelength is changed is an indication of quantum confinement. ${ }^{19,20}$ Such shifts result due to the excitation of different sizes of nanocrystals that have different optical energies. 


\subsubsection{Effect of capping agents on optical properties}

A silicon atom that is present in a silicon nanocrystal forms covalent bonds with four other silicon atoms. However, an atom on the surface, however, will have one or more valence electrons not bonded to $\mathrm{Si}$ atoms. If these valence electrons do not take part in a covalent bond with another material (e.g., hydrogen or carbon), these dangling bonds will give rise to localized defect states, providing sites for non-radiative recombination of an exciton (electron-hole pair), thus destroying the photoluminescence efficiency of the material. When such states exist, the material is said to be uncapped. These defect states might also be a source of photoluminescence along with bulk defects in the passivating material. Thus it is important to find a material that well passivates the nanocrystals and has a low bulk defect state density.

\begin{tabular}{|l|l|ll|l|l|}
\hline E.No & Precursor & \multicolumn{2}{|l|}{ Surfactant } & Reductant & Capping agent \\
\hline 2 & $\mathrm{SiCl}_{4}$ & $\begin{array}{l}\mathrm{C}_{12} \mathrm{E}_{5} \quad \text { (Pentaethylene glycol } \\
\text { monododecyl ether) }\end{array}$ & $\mathrm{LiAlH}_{4}$ & 1-Hexene \\
\hline 9 & $\mathrm{SiCl}_{4}$ & $\begin{array}{l}\mathrm{C}_{12} \mathrm{E}_{5} \quad \text { (Pentaethylene glycol } \\
\text { monododecyl ether) }\end{array}$ & $\mathrm{LiAlH}_{4}$ & 1-Heptene \\
\hline 10 & $\mathrm{SiCl}_{4}$ & $\begin{array}{l}\mathrm{C}_{12} \mathrm{E}_{5} \quad \text { (Pentaethylene glycol } \\
\text { monododecyl ether) }\end{array}$ & $\mathrm{LiAlH}_{4}$ & 1-Octene \\
\hline 11 & $\mathrm{SiCl}_{4}$ & $\begin{array}{l}\mathrm{C}_{12} \mathrm{E}_{5} \quad \text { (Pentaethylene glycol } \\
\text { monododecyl ether) }\end{array}$ & $\mathrm{LiAlH}_{4}$ & Allylamine \\
\hline
\end{tabular}

Table 3.4: List of capping agents used to modify the surface of Si nanocrystals 
Silicon nanocrystals were synthesized by the reaction of lithium aluminium hydride with silicon tetrachloride using $\mathrm{C}_{12} \mathrm{E}_{5}$ as surfactant. The hydride terminated particles were then surface modified with the capping agents mentioned in table 3.4. Silicon nanocrystals of size range 1.5-2 nm were used in this study. TEM studies indicated that the silicon nanocrystals size range was the same for all capping agents. The optical properties and the effect of different capping agents on the photoluminescence were studied. The alkyl capped particles were dispersed in the solvent n-hexane, while the amine capped particles were dispersed in methanol.

\section{UV-Vis and PL Studies of Alkyl and Amine Terminated Silicon Nanocrystals:}

Figure 3.11 shows the UV-Vis absorption spectra for the 1-hexene, 1-heptene and 1octene capped silicon nanocrystals. The alkyl terminated nanocrystals have absorption over a spectra range of $250-290 \mathrm{~nm}$. The peaks are centered around $265-270 \mathrm{~nm}$ in the absorption spectra and agrees relatively well with reports for $1-2 \mathrm{~nm}$ alkyl terminated silicon nanocrystals.

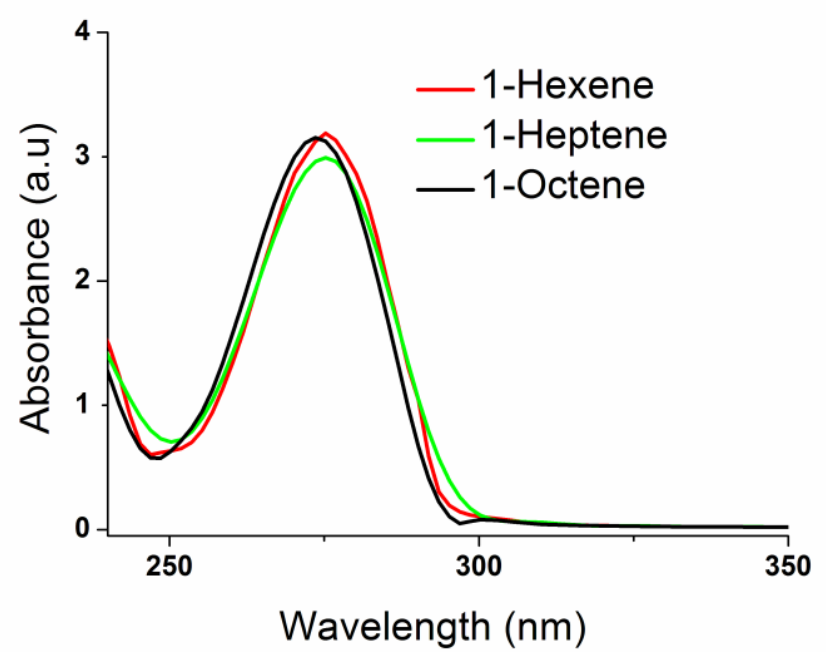

Figure 3.11: Absorption spectra of the alkyl capped silicon nanocrystals in hexane 


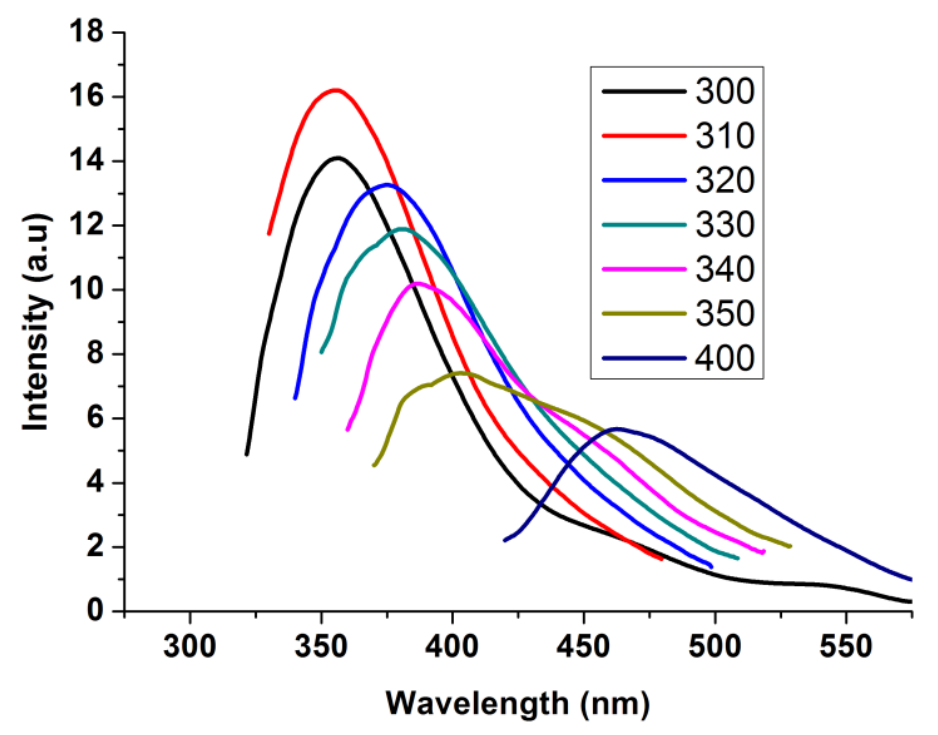

Figure 3.12: Photoluminescence spectra of 1-Hexene capped silicon nanocrystals in hexane

Figure 3.12 shows the photoluminescence spectra of 1-Hexene capped silicon nanocrystals in hexane. Photoluminescence spectra were obtained using wavelengths ranging from 300-400 nm. The photoluminescence of the 1-hexene capped silicon nanocrystal solution has a peak that ranges from $360 \mathrm{~nm}$ for the $300 \mathrm{~nm}$ excitation to $460 \mathrm{~nm}$ for the $400 \mathrm{~nm}$ excitation. 


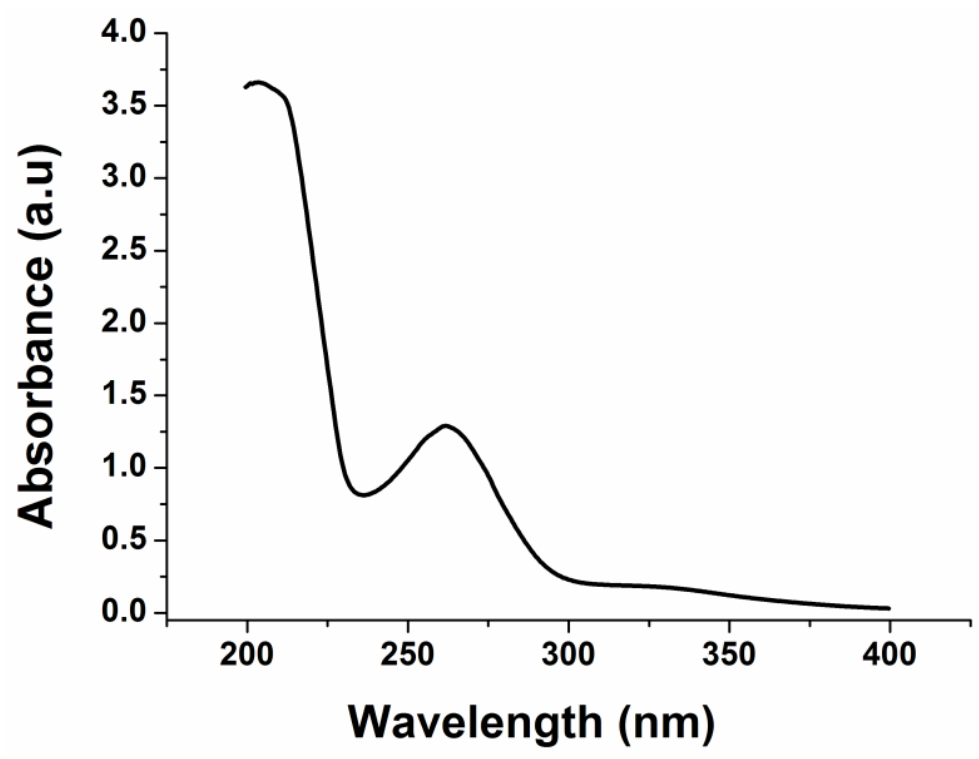

Figure 3.13: Absorption spectra of the amine capped silicon nanocrystals in methanol

Figure 3.13 shows the absorption spectra of amine capped silicon nanocrystals. The allylamine capped silicon nanocrystals exhibit absorption over a slightly greater spectral range (240-400) compared to the alkyl capped silicon nanocrystals. The amine capped particles exhibit a small shoulder at about $335 \mathrm{~nm}$ which is attributed to the absorption from the $\tau-\tau$ direct band gap transition. $5,16,21$ 


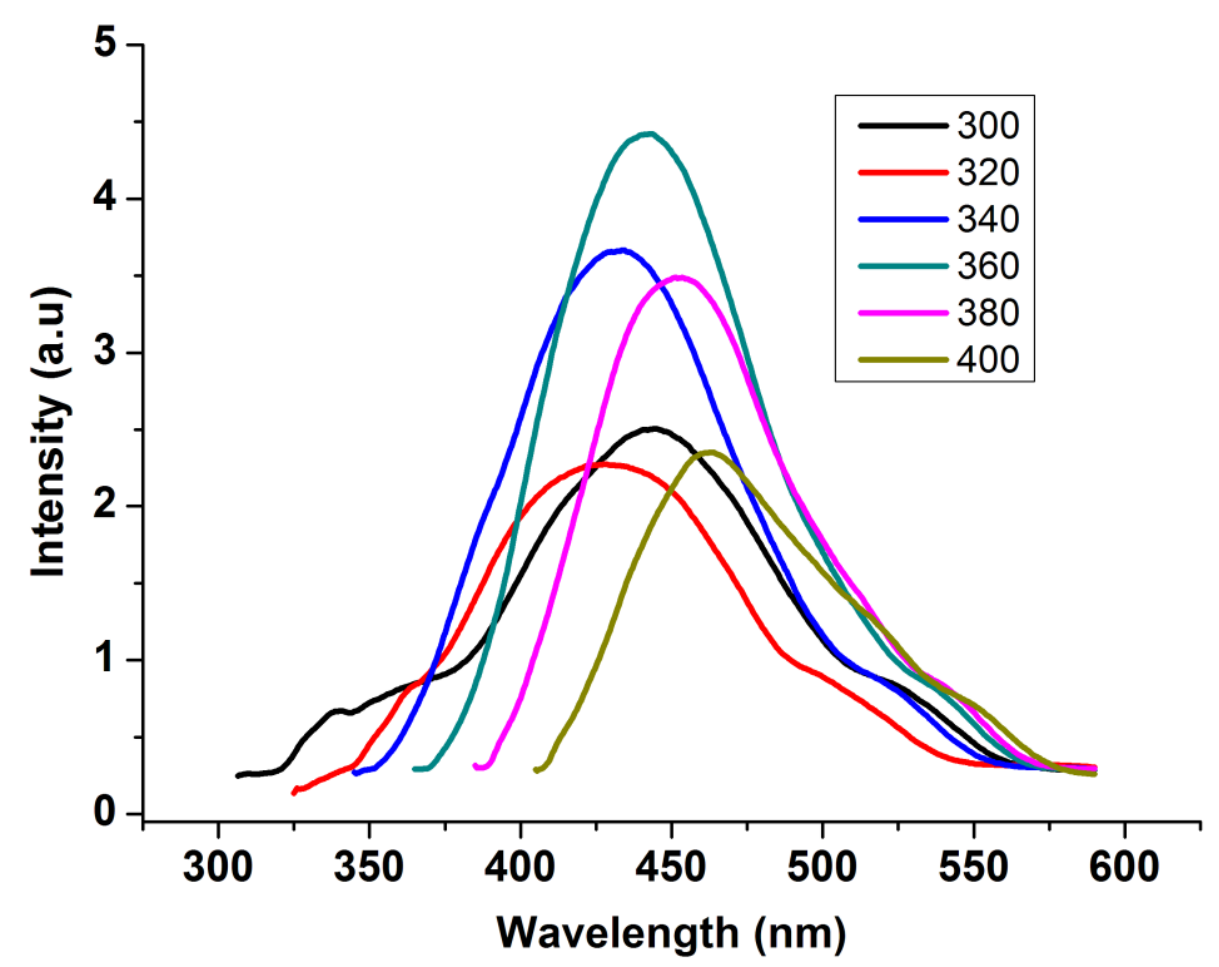

Figure 3.14: Photoluminescence spectra of amine capped silicon nanocrystals in methanol

Figure 3.14 shows the photoluminescence spectra of allylamine capped silicon nanocrystals in methanol. The PL spectra were obtained using excitation wavelengths ranging from 300 to $400 \mathrm{~nm}$ in $20 \mathrm{~nm}$ intervals. The photoluminescence of the allylamine-capped silicon nanocrystal solution has peaks ranging from $435 \mathrm{~nm}$ for the $320 \mathrm{~nm}$ excitation to $465 \mathrm{~nm}$ for the $400 \mathrm{~nm}$ excitation. This gives only a $35 \mathrm{~nm}$ red shift in the PL peak position, as the excitation wavelength is increased $80 \mathrm{~nm}$ from 320 to $400 \mathrm{~nm}$, possibly due to the presence of bigger nanocrystals.

There exists however a difference in optical response between the alkyl and amine terminated silicon nanocrystals. The photoluminescence spectra of the alkyl capped silicon nanocrystals displays small features within the main peak that are not observed 
in the allylamine-capped silicon nanocrystal PL spectra. The alkyl capped silicon nanocrystal solution also displays emission with higher energy than the allylaminecapped silicon nanocrystal solution for the same excitation wavelength. Since both solutions contained an identical size distribution of silicon nanocrystals, the vast difference in the PL spectra must be attributed to the effect of the surface-capping molecule and environment. This result provides direct evidence that the surface-capping molecule plays an important role in the radiative recombination mechanisms in silicon nanocrystals. ${ }^{18}$ In addition, the photoluminescence spectra in Figures 3.12 and 3.14 show no signs of emission at $600 \mathrm{~nm}$, which is typically associated with defects and $\mathrm{Si}-\mathrm{O}$ surface bonds. This shows that the silicon nanocrystals are well-passivated, robust, and highly crystalline. However, it is unlikely that all the silicon nanocrystal surface atoms are bonded to a surface passivating molecule (allylamine or alkene), and the small number of dangling silicon surface atoms probably form a $\mathrm{Si}-\mathrm{O}$ surface state. The PL spectra in both these figures show minimal emission from $\mathrm{Si}-\mathrm{O}$ surface bonds leading to the conclusion of a low defect density and a high percentage of surface passivation by the organic capping molecules. Solvents such as water and dichloromethane were each added to separate solutions of silicon nanocrystals, and no obvious shifts in the photoluminescence were detected. This indicates that the solvent does not play a major role in shifting the emission properties of silicon nanocrystals because of their well-passivated surfaces.

The blue photoluminescence from the allylamine and alkyl-capped silicon nanocrystals are in agreement with Zhou and co workers theoretical predictions. ${ }^{4}$ The difference in the optical response of the allylamine capped silicon nanocrystals and alkyl capped 
silicon nanocrystals is possibly due to the end groups attached to the capping agents as observed by Warner et al. Allylamine is a short-chain polar molecule, terminated by $\mathrm{NH}_{2}$, whereas the alkyl groups are short-chain nonpolar molecules with a $\mathrm{CH}_{3}$ termination.

The electronic charge distribution in the allylamine-capped silicon nanocrystals is modified by the polar nature of the allylamine in a similar way to that of a $\mathrm{Si}-\mathrm{O}$ surface bond in silicon nanocrystals. This may explain why the band gap of the allylaminecapped silicon nanocrystals is also slightly lower than that of the 1-heptene-capped silicon nanocrystals. Further Reboredo and co workers theoretical predictions on alkyl capped silicon nanocrystals suggest that the number of bound states increases as the length of the surface-capping molecule increases. ${ }^{23}$ They also suggest that the electronic structure of the 1-2 nm silicon nanocrystals changes because of the large overlap of these new states with the alkyl chains and the little overlap with the valence band wave functions localized in the silicon core, and the energy gap is not modified significantly. ${ }^{22}$ To conclude, the optical properties of silicon nanocrystals were found to be dependent on the nature of the capping agent. 


\subsubsection{Fourier Transform Infra-red Spectroscopy Studies}

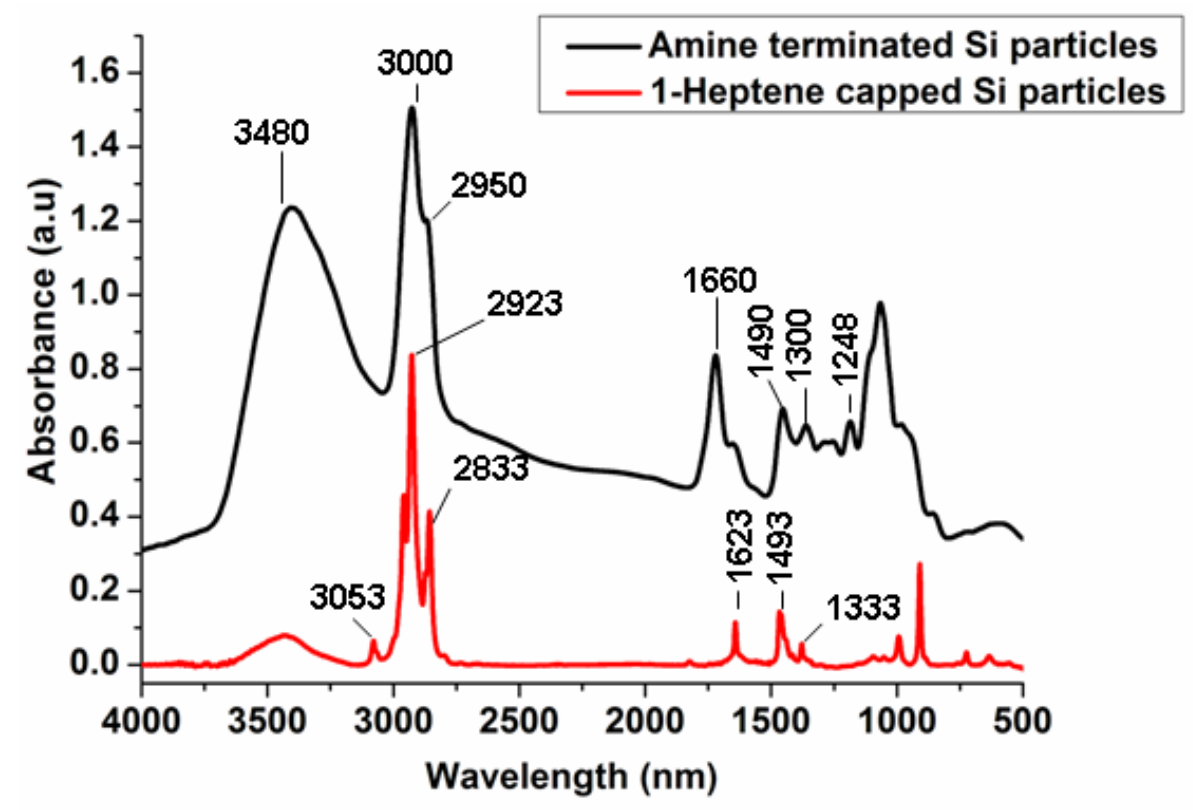

Figure 3.15: FTIR spectra of amine and 1-Heptene capped Si nanocrystals

Figure 3.15 is the FTIR spectra of amine (black) and 1-Heptene (red) capped silicon nanocrystals. The bonding of allylamine to the silicon nanoparticles was confirmed by FTIR spectroscopy. The peaks at 1490 and 1300 are attributed to the vibrational scissoring and symmetric bending of $\mathrm{Si}-\mathrm{CH}_{2}$. The absorbance between 2500 and $3500 \mathrm{~cm}^{-1}$ is attributed to symmetric and asymmetric vibrations of the $\mathrm{C}-\mathrm{CH}_{2}$ and $\mathrm{C}-\mathrm{NH}_{3}$ molecular components of allylamine. The peak at $1660 \mathrm{~cm}^{-1}$ is attributed to the allylamine and indicated its attachment to the silicon nanocrystals. The peaks between 1000 and 1100 $\mathrm{cm}^{-1}$ are attributed to the vibrational stretching of $\mathrm{Si}-\mathrm{OR}$, which is very small when compared to the FTIR reports of siloxane-coated silicon quantum dots, which have a total $\mathrm{Si}$ - OR surface coverage. ${ }^{21-23}$ This difference highlights the strength and stability 
of the $\mathrm{Si}-\mathrm{C}$ bond formed between the silicon quantum dots and the allylamine as well as the minimal number of $\mathrm{Si}$ - OR surface bonds present.

The bonding of the 1-Heptene to the silicon nanoparticles was also confirmed by FTIR spectroscopy. The peaks at $3053,2923,2833,1493$ and $1333 \mathrm{~cm}^{-1}$ correspond to the vibration peaks of the $-\mathrm{CH}_{3}$ and $-\mathrm{CH}_{2}$ groups. The presence of a small band between 1100 and $1000 \mathrm{~cm}^{-1}$ attributed to the $\mathrm{Si}-\mathrm{O}$ stretching mode indicates that though there is very minimum oxidation of the surface of the nanoparticles. 


\subsection{Discussion}

The effect of surfactants, reductants and precursors on particle size was studied in these set of experiments. While using the surfactants $\mathrm{TOAB}, \mathrm{C}_{12} \mathrm{E}_{5}$ and CTAB particles of size range $1.5-6 \mathrm{~nm}$ were synthesized. It was found that while using the cationic surfactants (CTAB and TOAB), though relatively monodisperse particles were obtained, the particles were not free standing and were embedded in surfactant. On the other hand while using the nonionic surfactant $\mathrm{C}_{12} \mathrm{E}_{5}$ as surfactant, the particles was found to be free standing and free of any organic impurities. The presence of organic impurities while using cationic surfactants and relatively low amounts of impurities on using a nonionic surfactant is attributed to the ease of purification. Whilst using silicon tetrabromide as precursor, nanocrystals of larger size were obtained, compare to silicon tetrachloride. This is attributed to the varied reactivity of the $\mathrm{Br}^{-}$ions in the solvent medium compared to that of $\mathrm{Cl}^{-}$.

The reducing agent $\mathrm{LiAlH}_{4}$ produced the smallest particles $(1.8 \pm 0.3 \mathrm{~nm})$. This is due to the reaction proceeding vigorously and quickly due to the strong reducing capability of $\mathrm{LiAlH}_{4}$. As a result of this rapid reduction there is a quick depletion of monomers with few monomers remaining for further growth of the nanocrystals, hence the small particle size. Slightly larger particle sizes are observed, if not significant for both $\mathrm{Li}(\mathrm{Et})_{3} \mathrm{BH}$ and $\mathrm{LiBH}_{4}$ on account of their slightly lowered reactivity. However on using $\mathrm{NaBH}_{4}$ as reductant particles of size $5 \mathrm{~nm}$ were synthesized. This is largely due to the weak reducing ability of sodium borohydride, which when injected into the solution, causes less 
monomer depletion in the nucleation burst leaving considerable unreacted monomer that contributes to nanocrystal growth causing the formation of nanoparticles of large size.

Three different silicon halide precursors were used to synthesize silicon nanoparticles. While silicon tetrachloride and silicon tetrabromide produced nanocrystals of size $1.8 \pm$ $0.3 \mathrm{~nm}$ and $4.5 \pm 0.5 \mathrm{~nm}$, the reaction using silicon tetraiodide did not produce any. The difference in particle size whilst changing precursors is attributed to the solventprecursor interactions. The lack of uniform solubility, of silicon tetraiodide, in the solvent was found to be the reason for not producing any nanocrystals. 


\subsection{Summary}

In this study, using inverse micelles, it was shown to be possible to produce monodisperse silicon nanocrystals of sizes. The effect of surfactants, reductants and precursors on particles size was studied along with their size dependent optical properties. It was found that $\mathrm{C}_{12} \mathrm{E}_{5}$ was an effective surfactant, which aided in the formation of free standing, impurity free silicon nanocrystals. Both lithium aluminium hydride and lithium triethyl borohydride were found to effective in producing nanoparticles of narrow size distribution. It was also found that using silicon tetrachloride smaller particles sizes could be made compared to silicon tetrabromide. Studies on the size control of silicon nanocrystals by solution methods using variables such as precursor, surfactants and reductants are few; these studies should help further knowledge about the synthetic aspects of silicon nanocrystals. Finally optical studies reveal the high monodispersity by displaying narrow fluorescence spectra at room temperature. 


\subsection{Experimental}

Synthesis: Alkyl-capped and amine capped silicon nanocrystals were made according to the following procedure. Dry toluene $(50 \mathrm{~mL})$ was made oxygen-free prior to use by 3 freeze-pump-thaw cycles, and put under $\mathrm{N}_{2}$ (using the Schlenk-line) during the whole synthesis. TOAB (Aldrich chemicals) $(0.5 \mathrm{~g}), \mathrm{C}_{12} \mathrm{E}_{5}$ (Nikko chemicals) $(0.5 \mathrm{~g})$ or CTAB (Aldrich) $(0.5 \mathrm{~g})$ was dispersed in the toluene by 20 min of mixing using a homogenizer. $\mathrm{SiCl}_{4}$ (Aldrich chemicals) $(0.1 \mathrm{~mL}), \mathrm{SiBr}_{4}$ (Aldrich chemicals) $(0.1 \mathrm{~mL})$ or $\mathrm{SiI}_{4}$ ((Aldrich chemicals $)(0.2 \mathrm{~g})$ was added via a gas-tight syringe. The reductants $\mathrm{LiAlH}_{4}(2 \mathrm{~mL})$, $\mathrm{Li}(\mathrm{Et})_{3} \mathrm{BH}(2 \mathrm{~mL}), \mathrm{LiBH}_{4}(2 \mathrm{ml})$ or $\mathrm{NaBH}_{4}(2 \mathrm{ml})$ were all obtained from Aldrich Chemicals was added to form hydrogen-terminated silicon nanocrystals in the micelles. Alkylterminated silicon nanocrystals were obtained by adding degassed and dry 1-Hexene (1 $\mathrm{mL})$, 1-Heptene $(1 \mathrm{ml})$ or Octene $(2 \mathrm{ml})$, while amine terminated silicon particles were obtained using allylamine (2ml). All capping agents were obtained from Aldrich Chemicals'. A catalytic amount of $0.05 \mathrm{M} \mathrm{H}_{2} \mathrm{PtCl}_{6}$ solution in isopropanol $(\sim 0.1 \mathrm{~mL})$ was added to the reaction mixture.

Purification: The resulting silicon nanocrystals were purified by removing solvents and other organic impurities by rotary evaporation, redispersing in suitable solvents and filtered through a hydrophilic $450 \mathrm{~nm}$ PVDF membrane filters (MILLEXHV, Millipore). The particles were then dissolved in hexane or methanol for characterization.

Characterization: All optical measurements were performed at standard pressure and room temperature, unless stated differently. Electronic absorption spectra were recorded 
in a quartz cuvette, using a Cary $1 \mathrm{UV}$-Vis double-beam spectrophotometer and were corrected for the solvent absorption. The scan range was $200-500 \mathrm{~nm}$ with a $600 \mathrm{~nm} / \mathrm{min}$ rate. Absorption spectra are corrected for hexane or methanol absorption, by subtracting. Photoluminescence spectra were obtained using a J-Y Fluorolog fluorescence spectrometer, with a slit width of $5 \mathrm{~nm}$ in both emission and excitation. FTIR measurements (Bruker, Vector 22 FTIR spectrometer), thin films of particle solutions in $\mathrm{CCl}_{4}$ were made between $\mathrm{NaCl}$ crystals and corrected for the background signal, by collecting 32 scans for each measurement. Transmission electron microscopy images and electron diffraction patterns were acquired digitally with a JEOL 2010 operated at an accelerating voltage of $200 \mathrm{KeV}$. EDX data were acquired on a JEOL 2010 equipped with an Oxford Inca EDS detector. 
125 


\subsection{References}

1. Veinot, J. G. C., Synthesis, surface functionalization, and properties of freestanding silicon nanocrystals, Chem. Comm. 2006, 4160-4168.

2. Hua, F.; Erogbogbo, F.; Swihart, M. T.; Ruckenstein, E., Organically capped silicon nanoparticles with blue photoluminescence prepared by hydrosilylation followed by Oxidation, Langmuir 2006, 22, 4363-4370.

3. Chao, Y. H., A.; Horrocks, B. R.; Hunt, M. R. C.; Poolton, N. R. J.; Yang, J.; Šiller, L., Optical luminescence from alkyl-passivated Si nanocrystals under vacuum ultraviolet excitation: origin and temperature dependence of the blue and orange emissions, Appl. Phys. Lett. 2006, 88, 263119-263121.

4. Zhou, Z. Y.; Brus, L.; Friesner, R., Electronic structure and luminescence of 1.1 and $1.4 \mathrm{~nm}$ silicon nanocrystals: oxide shell versus hydrogen passivation, Nano Lett. 2003, 3, 163-167.

5. Warner, J. H.; Hoshino, A.; Yamamoto, K.; Tilley, R. D., Water-soluble photoluminescent silicon quantum dots, Angew. Chem. Int. Ed. 2005, 44, 45504554. 
6. Rosso-Vasic, M.; Sprujit E.; Lagen B. V.; Cola, L.D.; Zuilhof, H., Alkylfunctionalized oxide-free silicon nanoparticles: synthesis and optical properties, Small, 2008, 10, 1835-1841.

7. (a) Sun, Q.-Y.D.; Van Lagen, B.; Giesbers, M.; Thuene, P. C.; Van Engelenburg, J.; De Wolf, F. A.; Zuilhof, H.; Sudhoelter, E. J. R., Covalently attached monolayers on crystalline hydrogen-terminated silicon: Extremely mild attachment by visible light, J. Am. Chem. Soc. 2005, 127, 2514-2523. ; (b) Sieval, A. B.; Vleeming, V.; Zuilhof, H.; Sudholter, E. J. R., An improved method for the preparation of organic layers of 1-alkenes on hydrogenterminated silicon surfaces, Langmuir 1999, 15, 8288-8291.

8. (a) Stewart, M. P. R., E. G.; Geders, T. W.; Allen, M. J.; Choi, H. C.; Buriak, J. M., Three methods for stabilization and functionalization of porous silicon surfaces via hydrosilylation and electrografting reactions, Phys. Stat. Sol. A: 2000, 182, 109-115.; (b) Buriak, J. M.; Stewart, M. P.; Geders, T. W.; Allen, M. J.; Choi, H. C.; Smith, J.; Raftery, D.; Canham, L. T. J., Lewis acid mediated hydrosilylation on porous silicon surfaces, J. Am. Chem. Soc. 1999, 121, 11491-11502. (c) Buriak, J. M., Silicon-carbon bonds on porous silicon surfaces, Adv. Mat. 1999, 11, 265-267. 
9. Liu, S.-M.; Yang, Y.; Sato, S.; Kimura, K., Enhanced photoluminescence from Si nano-organosols by functionalization with alkenes and their size evolution, Chem. Mater. 2006, 18, 637-642

10. Carter, R. S.; Harley, S. J.; Power, P. P.; Augustine, M. P., Use of NMR spectroscopy in the synthesis and characterization of air- and water-stable silicon nanoparticles from porous silicon, Chem. Mater. 2005, 17, 2932-2939.

11. (a) Jurbergs, D.; Rogojina, E.; Mangolini, L.; Kortshagen, U., Silicon nanocrystals with ensemble quantum yields exceeding 60\%, Appl. Phys. Lett. 2006, 88, 2331161-2331163. (b) Kortshagen, U.; Mangolini, L.; Bapat, A., Plasma synthesis of semiconductor nanocrystals for nano-electronics and luminescence applications, J. Nanoparticle Res. 2007, 9, 39-52.

12. Zou, J.; Sanelle, P.; Pettigrew, K. A.; Kauzlarich, S. M., Size and spectroscopy of silicon nanoparticles prepared via reduction of $\mathrm{SiCl}_{4}, J$. Clust. Sci. 2006, $17,565-578$.

13. Bley, R. A.; Kauzlarich, S. M., A low-temperature solution phase route for the synthesis of silicon nanoclusters, J. Am. Chem. Soc. 1996, 118, 12461-12462.

14. Neiner, D.; Chiu, H. W.; Kauzlarich, S. M., Low-temperature solution route to macrosopic amounts of hydrogen terminated silicon nanoparticles, J. Am. Chem. Soc. 2006, 128, 11016-11017. 
15. Zhang, X. M.; Neiner, D.; Wang, S. Z.; Louie, A. Y.; Kauzlarich, S. M., A new solution route to hydrogen-terminated silicon nanoparticles: synthesis, functionalization and water stability, Nanotechnology 2007, 18, 09560.

16. Neiner, D.; Kauzlarich, S. M., Hydrogen-capped silicon nanocrystals as a potential hydrogen storage material: Synthesis, characterization, and hydrogen release, Chem. Mater. 2010, 22(2), 487-493

17. Tilley, R. D. Warner, J. H.; Yamamoto, K.; Matsui, I.; Fujimori, H., Microemulsion synthesis of monodisperse surface stabilized silicon nanocrystals, Chem. Comm. 2005, 1833-1835.

18. Warner J. H.; Dunlop H-R.; Tilley R. D., Surface morphology dependent photoluminescence from colloidal silicon nanocrystals, $J$ Phys Chem $B$ 2005, 109(41), 19064-19067.

19. Taylor, B. R.; Kauzlarich, S. M.; Delgado, G. R.; Lee, H. W. H., Solution synthesis of quantum confined Ge nanoparticles, Chem. Mater. 1999, 11, 2493.

20. Taylor, B. R.; Kauzlarich, S. M.; Delgado, G. R.; Lee, H. W. H., Solution synthesis of germanium nanocrystals demonstrating quantum confinement, Chem. Mater. 1998, 10, 22. 
21. Wilcoxon, J. P.; Provencio, P. P.; Samara, G. A., Optical and electronic properties of Si nanoclusters synthesized in inverse micelles, Phys. Rev. B 1999, 60, 2704.

22. Puzder, A.; Williamson, A. J.; Grossman, J. C.; Galli, G., Computational Studies of the Optical Emission of Silicon Nanocrystals, J. Am. Chem. Soc. 2003, 125, 2786.

23. Reboredo, F. A.; Galli, G., Theory of alkyl-terminated silicon quantum dots, $J$. Phys. Chem. B 2005, 109, 1072.

24. Baldwin, R. K.; Pettigrew, K. A.; Ratai, E.; Augustine, M. P.; Kauzlarich, S. M., Solution reduction synthesis of surface stabilized silicon nanoparticles Chem. Commun. 2002, 1822. 


\section{Solution Synthesis and Characterization of Germanium Nanocrystals}

\subsection{Introduction}

This chapter describes the solution synthesis of germanium nanocrystals by microemulsion and high temperature routes, summarizing the procedures which have yielded the best quality germanium nanocrystals to date, and their characterization. This work began with the search for a method to produce large amounts of germanium nanocrystals, and exercise more control over their properties, than is possible by previously reported literature methods. The initial work on germanium demonstrated that water-soluble photoluminescent nanocrystals could be made by the hydride reduction of $\mathrm{GeCl}_{4}$ in inverse micelles, and demonstrated excellent stability when surface terminated with an amine group. Continuing work on the hydride reduction of $\mathrm{GeCl}_{4}$ in inverse micelles has demonstrated the utility of this method in preparing crystalline germanium nanocrystals in useful quantities, with excellent stability, photoluminescence, and some provision for size control. Though many synthetic approaches have been reported for the synthesis of Germanium nanoparticles, one of the primary challenges are the identification of appropriate precursors that would readily decompose or undergo reduction to sustain controlled crystalline particle growth.

${ }^{16}$ Portions of this chapter have been previously published as Sujay Prabakar, Amane Shiohara, Sanshiro Hanada, Kouki Fujioka, Kenji Yamamoto, and Richard D Tilley; Chemistry of Materials, 2010, 22(2), pp 482-486. 
As with the case in silicon, germanium particles have also been synthesized commonly by solution reduction routes. Germanium nanocrystals have previously been produced using different methods. Veinot et al. produced alkyl-surface functionalized germanium nanocrystals via metal hydride reduction of nonpolar solutions of CTAB and $\mathrm{GeI}_{4}$ at room temperature. ${ }^{1}$ Kauzlarich et al. investigated the synthesis of germanium nanoparticles by the sodium naphthalide reduction of $\mathrm{GeCl}_{4}$ varying reactions conditions. ${ }^{2}$

Korgel et al. formed high yields of Ge nanocrystals by the reduction of $\mathrm{GeI}_{4}$ by $\mathrm{LiAlH}_{4}$ in high boiling point solvents. ${ }^{3}$ Supercritical solvents have also been used to synthesize reasonable yields of germanium nanocrystals. ${ }^{4-6}$ The size controlled syntheses of monodisperse germanium nanocrystals using colloidal routes that involve high reaction temperature are well established. However, less progress has been made in the low temperature synthesis of Ge nanocrystals mainly because of its covalency. ${ }^{7}$ Experiments 1-4 of this chapter deals with the size controlled synthesis of germanium nanocrystals using hydride reducing agents. Experiment 5 in particular talks about size control by varying precursor concentrations.

Further to facilitate easy isolation of the particle from excess surfactant, $\mathrm{C}_{12} \mathrm{E}_{5}$ was used as the surfactant of choice, after results obtained in chapter 1. Germanium nanocrystals are expected to exhibit photoluminescence in the visible region with reasonable quantum yields, due to the large excitonic Bohr radius $(\mathrm{Rb}=11.5 \mathrm{~nm})$ and have been proposed for use in different applications because of their unique physical properties. ${ }^{8,9}$ However, reports on germanium nanocrystals as biological tools/labels are few and 
limited. For example, Boyle et al. reported the synthesis of $\sim 3-5 \mathrm{~nm}$ phospholipidencapsulated water-soluble germanium nanocrystals that were used as biomarkers for cell signaling in RBL-2H3 cells in vitro. ${ }^{10}$ For germanium nanocrystals to be used in biological applications, photoluminescence quantum yield in the visible region and water-solubility are vital. Thus the development of synthetic methods for producing germanium nanocrystals with controlled sizes and modified surfaces, with low toxicity, is important for the biological applications of germanium nanocrystals.

Thermal reduction of precursors to form nanocrystalline germanium particles have also been reported in the literature. Korgel et al used tetraethylenegermane (TEG) or diphenylgermane (DPG) in the presence of octanol and supercritical $\mathrm{CO}_{2}$ to synthesize highly crystalline germanium particles of $\mathrm{d}=5.6-10.1 \mathrm{~nm} \cdot{ }^{4-6}$ Gerung et al used an amido based germanium precursor $\mathrm{Ge}\left[\mathrm{N}\left(\mathrm{SiMe}_{3}\right)_{2}\right]_{2}$ in 1 -octadecene at $300^{\circ} \mathrm{C}$ using oleylamine as surfactant to prepare particles with an average particle size of $7 \pm 4 \mathrm{~nm} .{ }^{11}$

In addition to a room temperature micro-emulsion synthesis, high temperatures techniques were explored to provide alternate routes to high yielding and photoluminescing Ge nanocrystals. This section of this chapter presents, in particular the high temperature reduction of $\mathrm{GeCl}_{4}$ by sodium borohydride in high boiling point solvents. 


\subsection{The Microemulsion Synthesis of Germanium Nanocrystals}

\subsubsection{Effect of Reducing agents on Particle Size (Expt.1-4)}

Experiments 1-4 of this chapter deals with the size controlled synthesis of germanium nanocrystals using hydride reducing agents. Table 4.1 lists the four different hydride reducing agent used in this study, the reaction parameters and the respective particle sizes of the synthesized particles.

\begin{tabular}{|l|l|l|l|l|l|}
\hline $\begin{array}{l}\text { Exp. } \\
\text { No }\end{array}$ & Reductant & Surfactant & Solvent & Precursor & $\begin{array}{l}\text { Particle } \\
\text { Sizes }\end{array}$ \\
\hline 1 & $\mathrm{LiALH}_{4}$ & $\mathrm{C}_{12} \mathrm{E}_{5}$ & Hexane & $\mathrm{GeCl}_{4}$ & $4.0 \pm 0.5 \mathrm{~nm}$ \\
\hline 2 & $\mathrm{Li}(\mathrm{Et})_{3} \mathrm{BH}$ & $\mathrm{C}_{12} \mathrm{E}_{5}$ & Hexane & $\mathrm{GeCl}_{4}$ & $4.5 \pm 0.5 \mathrm{~nm}$ \\
\hline 3 & $\mathrm{LiBH}_{4}$ & $\mathrm{C}_{12} \mathrm{E}_{5}$ & Hexane & $\mathrm{GeCl}_{4}$ & $5.5 \pm 0.5 \mathrm{~nm}$ \\
\hline 4 & $\mathrm{NaBH}_{4}$ & $\mathrm{C}_{12} \mathrm{E}_{5}$ & Hexane & $\mathrm{GeCl}_{4}$ & $25.0 \pm 0.5 \mathrm{~nm}$ \\
\hline
\end{tabular}

Table 4.1: List of hydride reducing agents used to synthesize germanium nanocrystals

It has been previously shown that by using reducing agents with different reactivities,

the size of the resulting nanocrystals can be controlled. ${ }^{2}$ To compare the effect of reductants in this study, four experiments were conducted. The first used $\mathrm{LiAlH}_{4}$ as reductant, primarily because of its powerful reducing capability and also since it has been previously shown to produce nanocrystals of narrow size distribution. Additionally 
$\mathrm{Li}\left(\mathrm{C}_{2} \mathrm{H}_{5}\right)_{3} \mathrm{BH}$ (superhydride), $\mathrm{LiBH}_{4}$ and $\mathrm{NaBH}_{4}$ were used with the strength of the reducing agents decreasing along this list with $\mathrm{LiAlH}_{4}$ being the most powerful and $\mathrm{NaBH}_{4}$ the weakest. In this study, all other experimental variables were kept constant and the samples were reacted for 3 hours at room temperature, with the reducing agents added in one swift injection.

Figure 4.1 shows low-resolution transmission electron microscope (TEM) images of a number of allylamine capped germanium nanocrystals. The images illustrate that the selective use of the hydride reducing agents can control the size of the resulting Ge nanocrystals. 


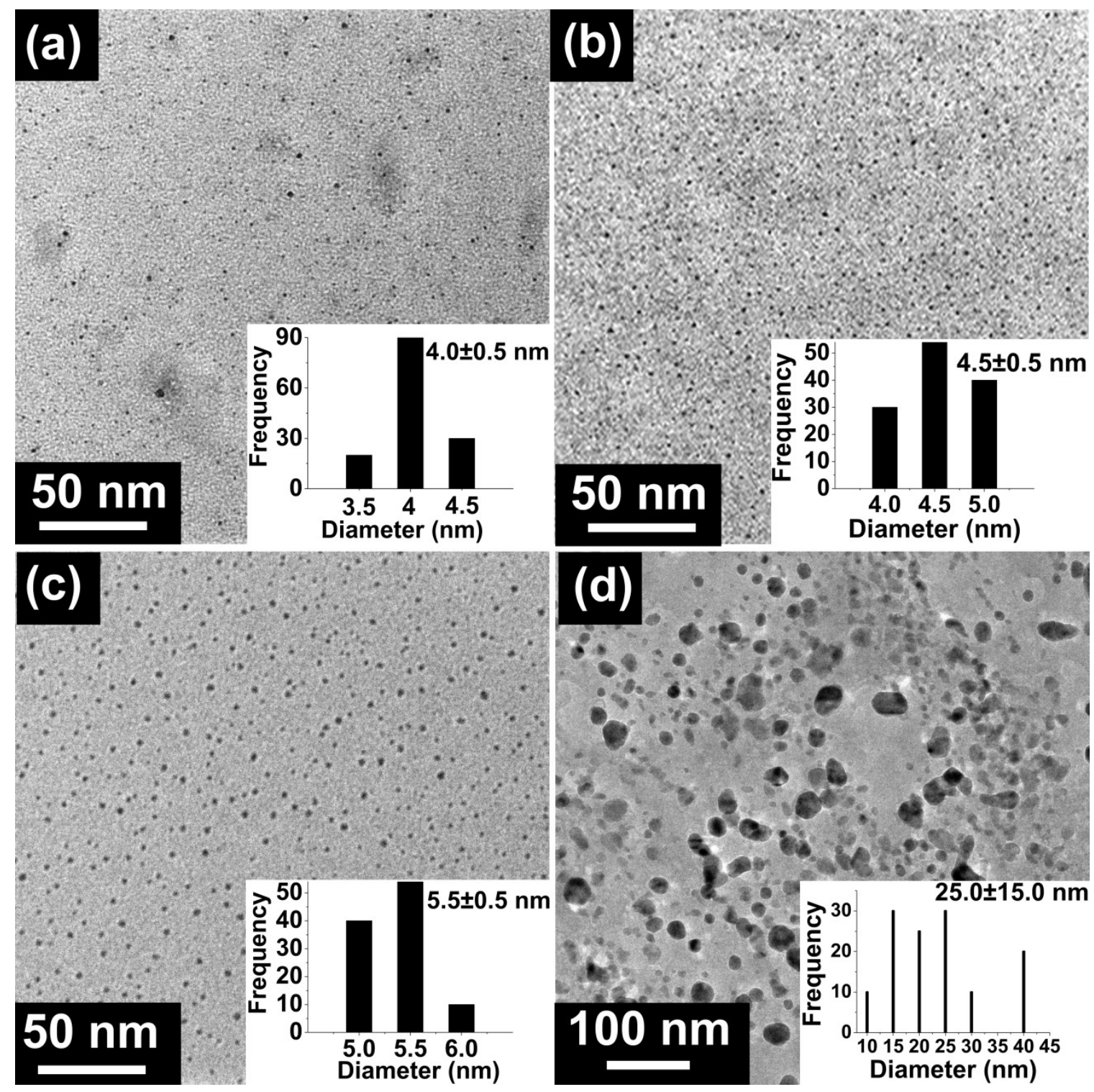

Figure 4.1: TEM images of Ge nanocrystals obtained from reduction by (a) $\mathrm{LiAlH}_{4}$ and (b) $\mathrm{Li}\left(\mathrm{C}_{2} \mathrm{H}_{5}\right)_{3}$, (c) $\mathrm{LiBH}_{4}$, (d) $\mathrm{NaBH}_{4}$.

Figure 4.1a is low resolution TEM image of germanium nanocrystals obtained from the reduction using $\mathrm{LiAlH}_{4}$. The nanocrystals $(4.0 \pm 0.5 \mathrm{~nm})$ are predominantly spherical and particles of sizes 3.5, 4.0 and $4.5 \mathrm{~nm}$ were formed (based on 300 particles). Figure 4.1b and $\mathrm{C}$ are low resolution TEM images of germanium nanocrystals obtained from 


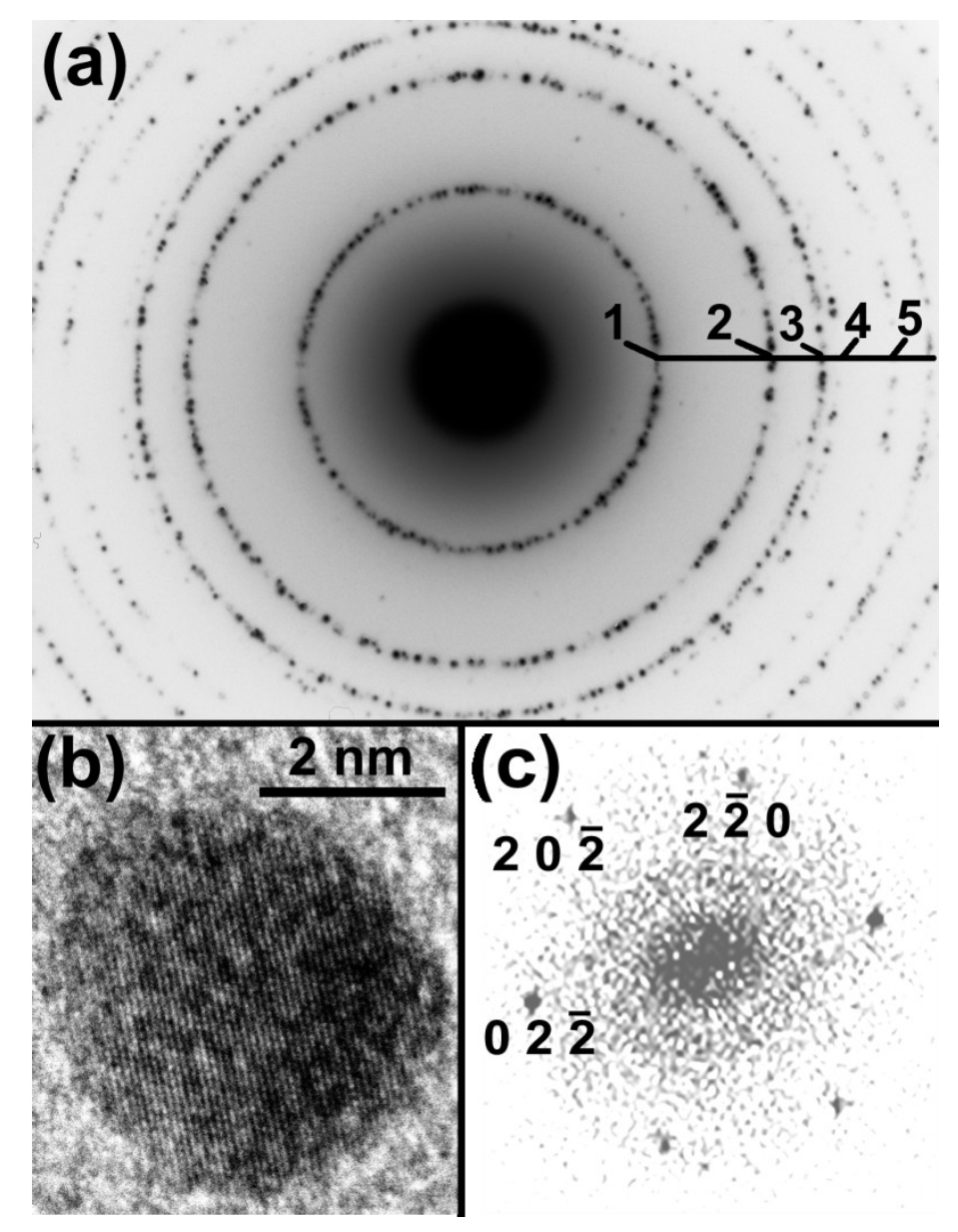

Figure 4.2: (a) Selected-area electron diffraction pattern (SAED) for Ge nanocrystals obtained from the hydride reduction of $\mathrm{GeCl}_{4}$, rings matched the diamond germanium planes: Ring $1=(111) 2=(220) 3=(311) 4=(400)$ and $5=(331)$, (b) high-resolution TEM image of an amine-terminated Ge nanocrystal synthesized using $\mathrm{LiAlH}_{4}$ as reductant, viewed down the [111] direction (c) corresponding fast Fourier transform (FFT) of the image.

the reduction using $\mathrm{Li}(\mathrm{Et})_{3} \mathrm{BH}$ and $\mathrm{LiBH}_{4}$ respectively. The nanoparticles were again found to be predominantly spherical and relatively monodisperse with sizes $4.5 \pm 0.5$ $\mathrm{nm}$ (based on 500 particles) and $5.5 \pm 0.5 \mathrm{~nm}$ (based on 250 particles) respectively. The 
decreasing reactivity's of the hydride reducing agents $\mathrm{s}$ attributed to the various particle sizes. Figure 4.1d is a low resolution TEM image of germanium nanocrystals obtained from the reduction using $\mathrm{NaBH}_{4}$. The nanoparticles were found to be larger in size and were irregularly shaped nanocrystals of size $25.0 \pm 15.0 \mathrm{~nm}$ (based on 300 particles). This trend is attributed to the weak reducing capability of $\mathrm{NaBH}_{4}$.

Confirmation of the crystal structure of the germanium nanocrystals was obtained from selected-area electron diffraction (SAED) and is shown in Figure 4.2a. The SAED rings matched well with the diamond crystal structure adopted by germanium. Figure $4.2 \mathrm{~b}$ is a high-resolution TEM image (HRTEM) of a $4 \mathrm{~nm}$ germanium nanocrystal obtained from the reaction using $\mathrm{LiAlH}_{4}$ as reductant. The nanocrystal is highly crystalline with the lattice fringes clearly observable. The fast Fourier transform of the HRTEM image in Figure $4.2 \mathrm{c}$, exhibits the characteristic pattern of the diamond germanium crystal structure, viewed down the [111] direction. Crystalline nanocrystals of germanium such as these were obtained for all hydride reducing agents. 


\subsubsection{Effect of Precursor Concentration on Particle Size (Expt. 5)}

To investigate the effect of precursor $\left(\mathrm{GeCl}_{4}\right)$ concentration on the formation of $\mathrm{Ge}$ nanocrystals, experiments using concentrations ranging from $0.46 \mathrm{mmol}$ (low concentration) to $1.38 \mathrm{mmol}$ (high concentration) were used. Pentaethylene glycol monodecyl ether $\left(\mathrm{C}_{12} \mathrm{E}_{5}\right)$ was used as surfactant. After the lithium aluminium hydride was added to the germanium tetrachloride the reaction proceeded to give a cloudy yellow solution after all gaseous species have escaped. A white precipitate similar to that observed for experiment 1 was observed for all concentrations. Allylamine was added to cap the hydride-terminated germanium nanocrystals rendering them hydrophobic and also prevent the nanocrystals from oxidizing. A platinum catalyst $\left(\mathrm{H}_{2} \mathrm{PtCl}_{6}\right)$ was used to

catalyze the hydro-germylation process. Yellow colored oils that were soluble in polar solvents were obtained for all concentrations after purification. At higher concentrations yellow precipitates were obtained that could be purified by centrifugation and resupension were obtained. EDX analysis confirmed the presence of Ge for all samples (Figure 4.3).

Figure 4.4 shows two typical results. At low concentration $(0.46 \mathrm{mmol})$ Ge nanocrystals with an average size of $4.0 \pm 1.08 \mathrm{~nm}$ are synthesized (shown earlier in figure 4.1a). When doubling the amount of precursor to $0.93 \mathrm{mmol}$ Ge nanocrystals of particle size $5 \pm 0.5 \mathrm{~nm}$ were synthesized. However at higher concentrations $(1.39 \mathrm{mmol})$ an average size of $7 \pm 2.0 \mathrm{~nm}$ (Figure $4.4 \mathrm{~b}$ ) was observed. Figure $4.4 \mathrm{c}$ is a plot of particle size as a function of concentration. From this plot it is clear that smaller size distributions are 
obtained at low concentrations. At concentrations higher than $1.8 \mathrm{mmol}$, the particles were observed to form Ge clusters. These observations are consistent with the La Mer and Dinegar's theory ${ }^{18}$ that, a higher amount of particles will be polydisperse and exhibit non-uniform growth when the initial solutions are highly concentrated.

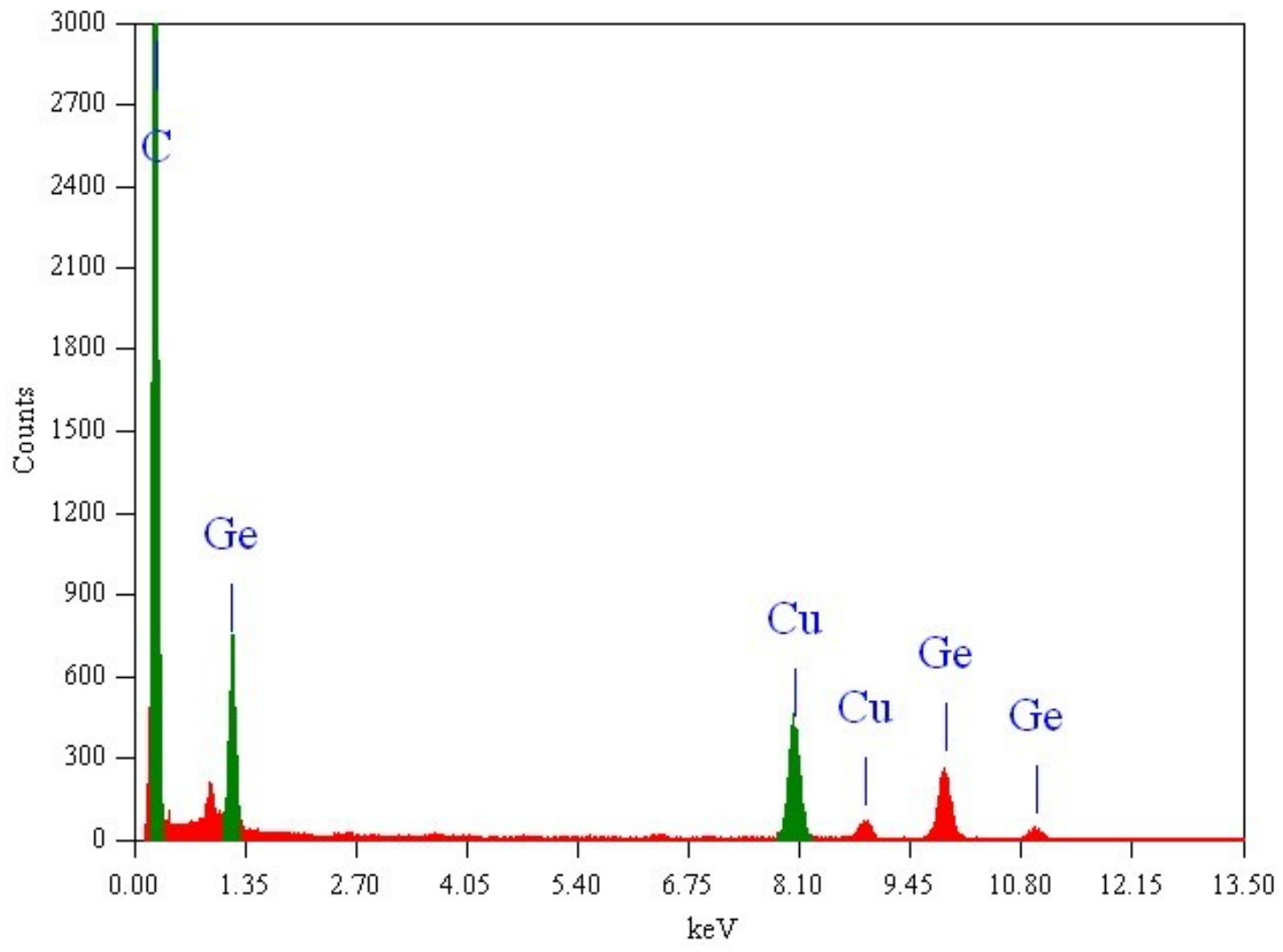

Figure 4.3: An EDX spectrum of germanium nanocrystals synthesized by the reduction of germanium tetrachloride by lithium aluminium hydride 

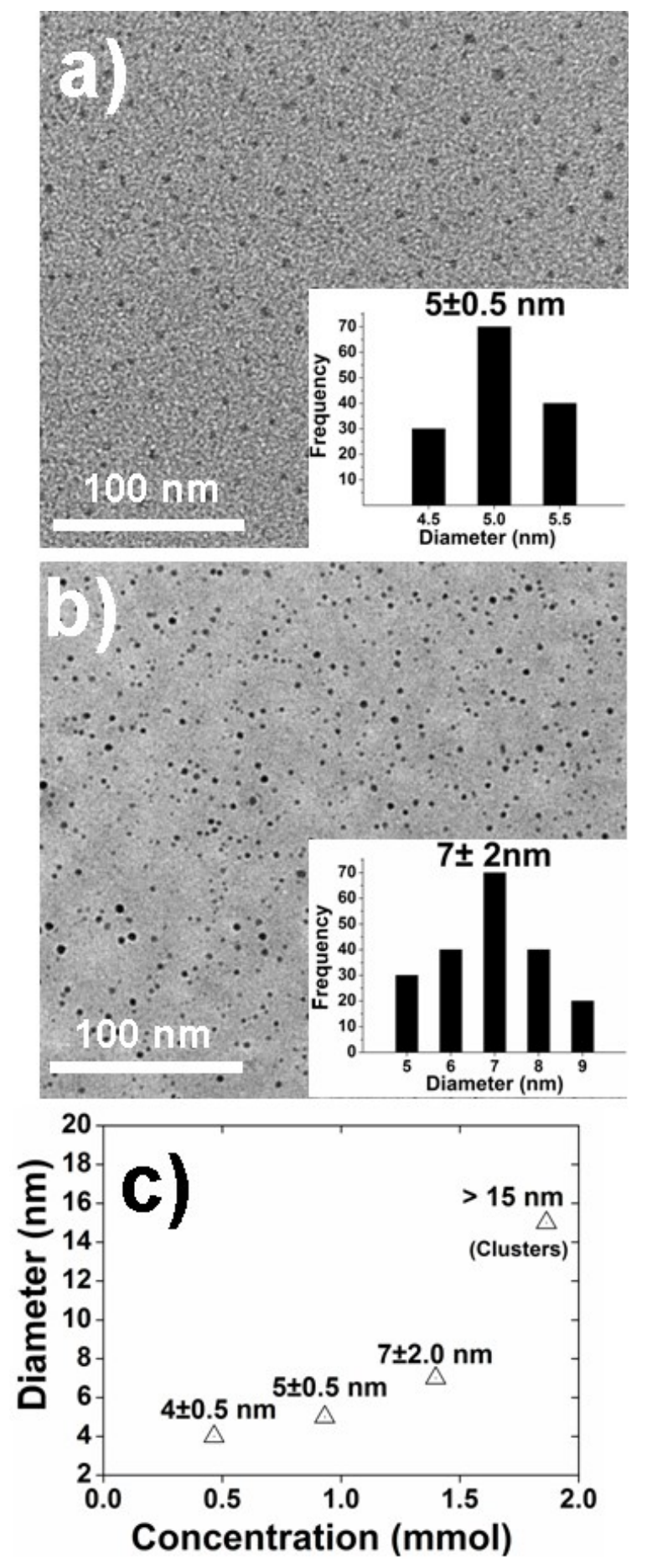

Figure 4.4: TEM images of Ge nanocrystals prepared from (A) $(0.93 \mathrm{mmol})$ and (B) (1.39 mmol) precursor concentrations. Insets show size distribution. (C) Plot of Ge nanocrystal size as a function of precursor concentration. 


\subsubsection{Spectroscopic Evidence of Quantum Confinement}

Quantum confinement results in optical properties that are highly size-dependent, e.g., smaller nanocrystals absorb and emit light at higher energies than larger nanocrystals and have large oscillator strengths. While bulk Ge absorbs weakly in the infra-red, nanocrystals produced by this method absorb strongly in the visible and ultraviolet region.

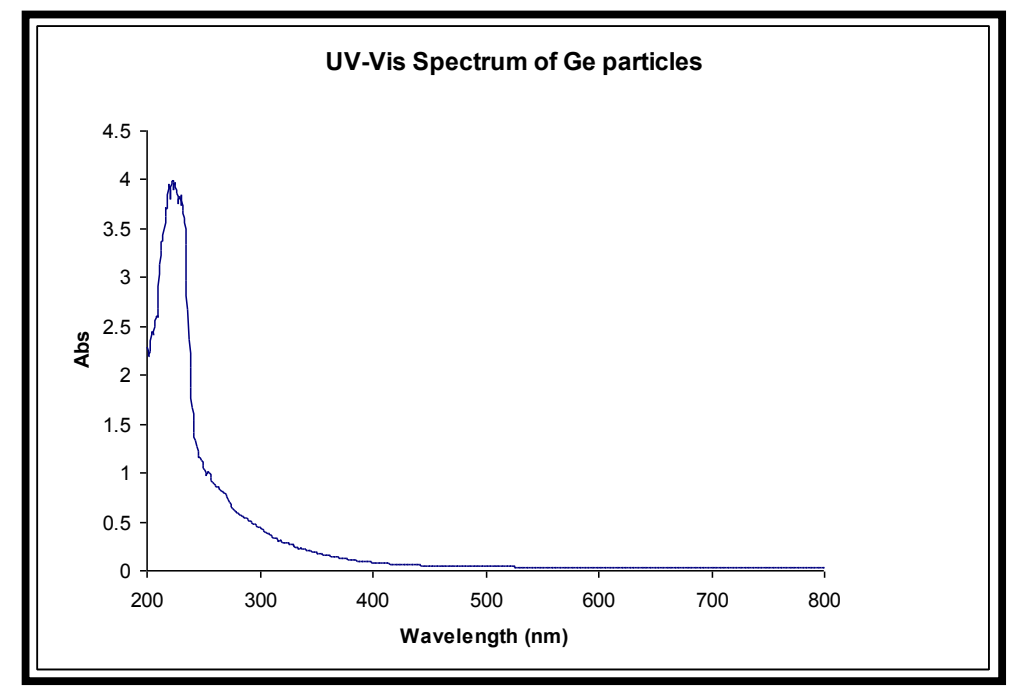

Figure 4.5: UV-vis absorption spectra of Ge nanocrystals in methanol

Figure 4.5 shows UV-vis absorption spectra of Ge nanocrystals of approximately $5 \mathrm{~nm}$ mean diameter produced from the reaction using $\mathrm{LiBH}_{4}$ as reductant. The spectra show an absorption onset considerably above the bandgap of bulk of Ge, suggesting that these nanocrystals are quantum confined. The effect of solvent on the optical properties of nanocrystals is also an important issue. When the solvent was changed from methanol to water, the same results were obtained suggesting that re-suspending the solvents in 
different solvents does not change the UV-vis absorption spectrum. Thus the UV-vis absorption spectra of these nanocrystals reflect the properties of the Ge nanocrystalline core and are largely insensitive to solvent effects.

Figure 4.6 shows the $400 \mathrm{~nm}$ photoluminescence spectra (normalized) of an aqueous solution of allylamine-capped Ge nanocrystals of sizes 4.0, 4.5 and $5.5 \mathrm{~nm}$ made using $\mathrm{LiAlH}_{4}, \mathrm{Li}\left(\mathrm{C}_{2} \mathrm{H}_{5}\right)_{3} \mathrm{BH}$ (superhydride) and $\mathrm{LiBH}_{4}$ respectively. Emission across a relatively narrow region of 420-480 nm is observed with full width maximums (FWHM) of $70-80 \mathrm{~nm}$ indicating the monodisperse nature of the Ge nanocrystals. The PL peaks between 420 to $480 \mathrm{~nm}$ are similar to previous reports of Ge nanocrystals. ${ }^{11,12}$

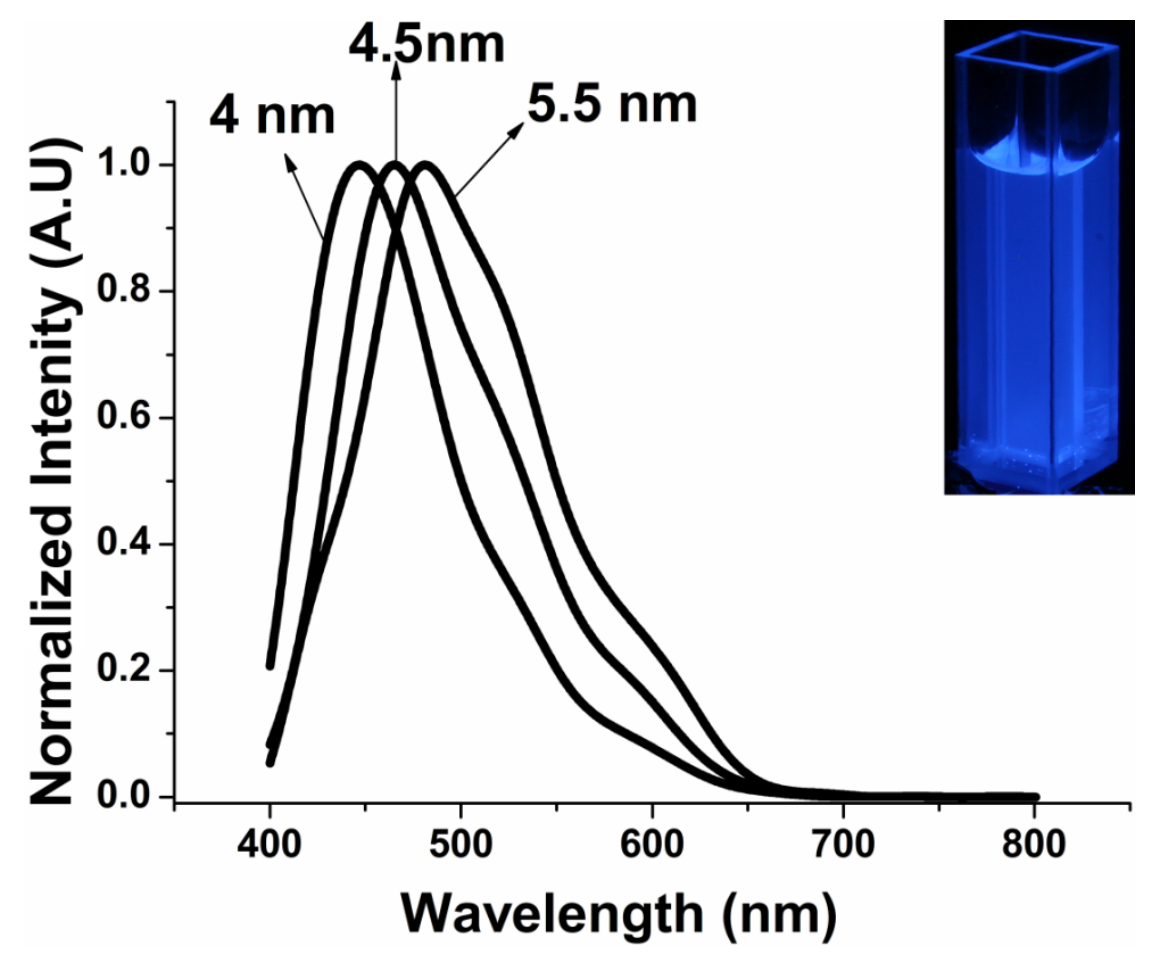

Figure 4.6: Photoluminescence spectra of amine-terminated Ge nanocrystals excited at $400 \mathrm{~nm}$. The inset shows the fluorescence of the germanium nanocrystals excited under an UV lamp. 
As expected, no photoluminescence from direct band gap transitions was detected from the nanocrystals of average size $25 \mathrm{~nm}$, and were too big to be in the quantum confinement regime. These results are important as they illustrate the ability to control nanoparticle size in the quantum confinement regime leading to the Ge nanocrystals exhibiting tunable photoluminescence in the visible wavelength region via direct band gap transitions. $^{13-16}$

\subsubsection{Fourier Transform Infra-red Spectroscopy}

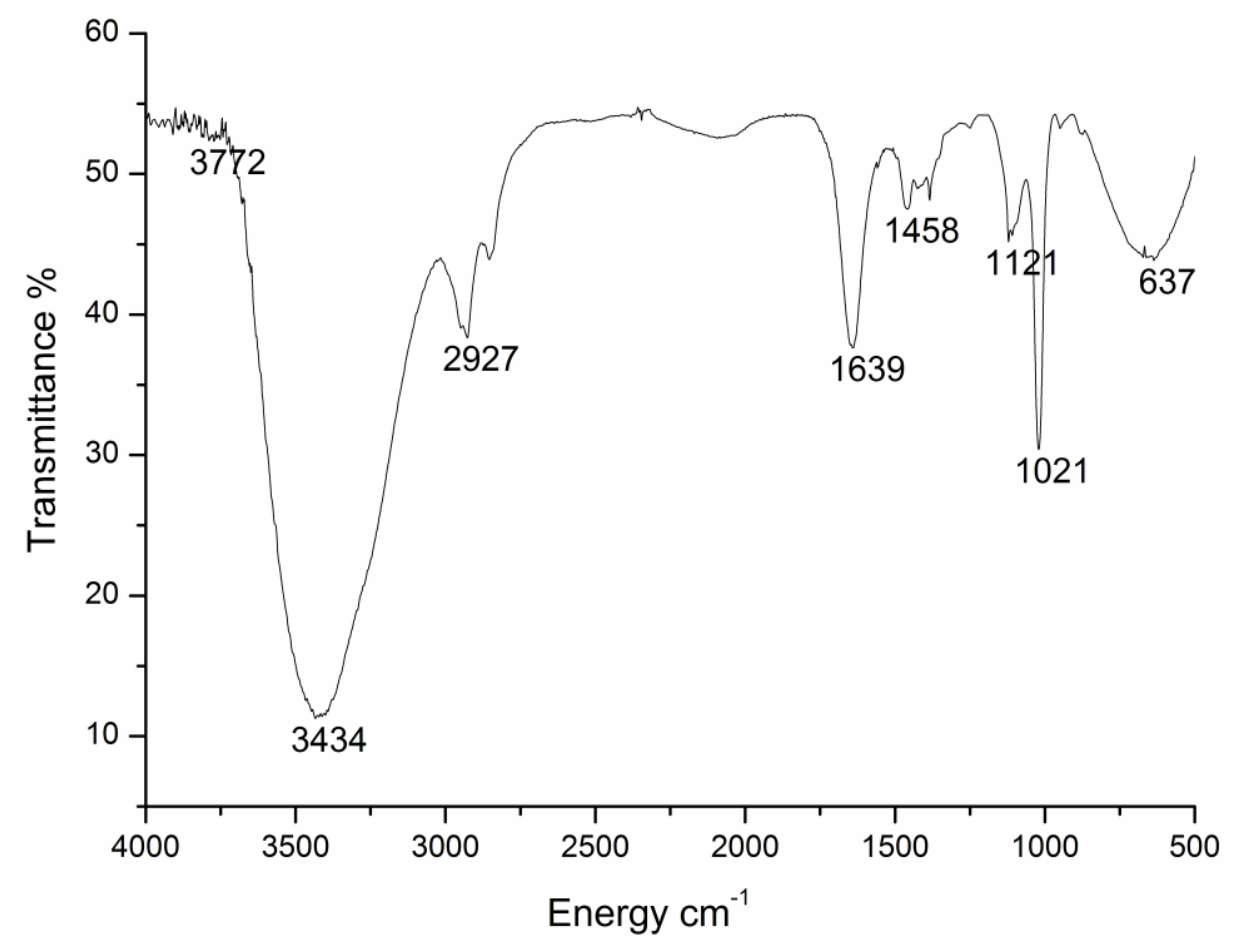

Figure 4.7: FTIR pattern of amine capped germanium nanocrystals

The bonding of allylamine to the surface of the silicon quantum dots was confirmed by

FTIR spectroscopy (figure 4.7). Peaks were observed at 1458 and $1121 \mathrm{~cm}^{-1}$ and attri- 
buted to the vibrational scissoring and symmetric bending of $\mathrm{Ge}-\mathrm{CH}_{2}$. The absorbance between 2500 and $3500 \mathrm{~cm}^{-1}$ is attributed to symmetric and asymmetric vibrations of the $\mathrm{C}-\mathrm{CH}_{2}$ and $\mathrm{C}-\mathrm{NH}_{3}$ molecular components of the allylamine, while the dominant peak at $1639 \mathrm{~cm}^{-1}$ is attributed to the allylamine and clearly indicates its attachment to the silicon quantum dots. The peaks between 1000 and $1100 \mathrm{~cm}^{-1}$ is attributed to the vibrational stretching of Ge-O. 


\subsection{High Temperature Synthesis of Germanium Nanocrystals}

\subsubsection{Reactions of Germanium Tetrachloride and Sodium Borohydride in 1-octadecene and 1-octadecene/oleic acid at High Temperatures (Experiments 6 and 7)}

The aim of this experiment was to develop a synthesis which could produce high yields of photoluminescing germanium nanocrystals. Sodium borohydride in triglyme was used as the reductant to facilitate high temperature reduction and facile crystallization. Sodium borohydride $\left(\mathrm{NaBH}_{4}\right)$ in triglyme was injected into a degassed solution of either 1-octadecene or 1-octadecene/oleic acid mixture and heated up to $200^{\circ} \mathrm{C}$. $\mathrm{GeCl}_{4}$ is dissolved in either of the two solvent systems in a $\mathrm{N}_{2}$ filled glove box and transferred into an airtight syringe, which is then injected into the reaction mixture and allowed to react for 10 minutes before the reaction mixture is gradually heated to $300^{\circ} \mathrm{C}$ and refluxed at that temperature for $3 \mathrm{~h}$. On injection the colour of the reaction mixture turns reddish brown and becomes dark brown on reacting after $3 \mathrm{~h}$. The resulting brown powder was washed with excess toluene and ethanol and centrifuged to remove byproducts. Figure 4.8 is the TEM image of the as-synthesized product obtained whilst using 1-octadecene as the solvent. The reaction failed to produce monodisperse nanocrystals; instead bulk crystalline material aggregating into clusters of a few hundred nanometers was synthesized. An XRD of the brown powder corresponded to the di- 
amond cubic crystal structure of germanium. The peaks denoted by * were from impurities.
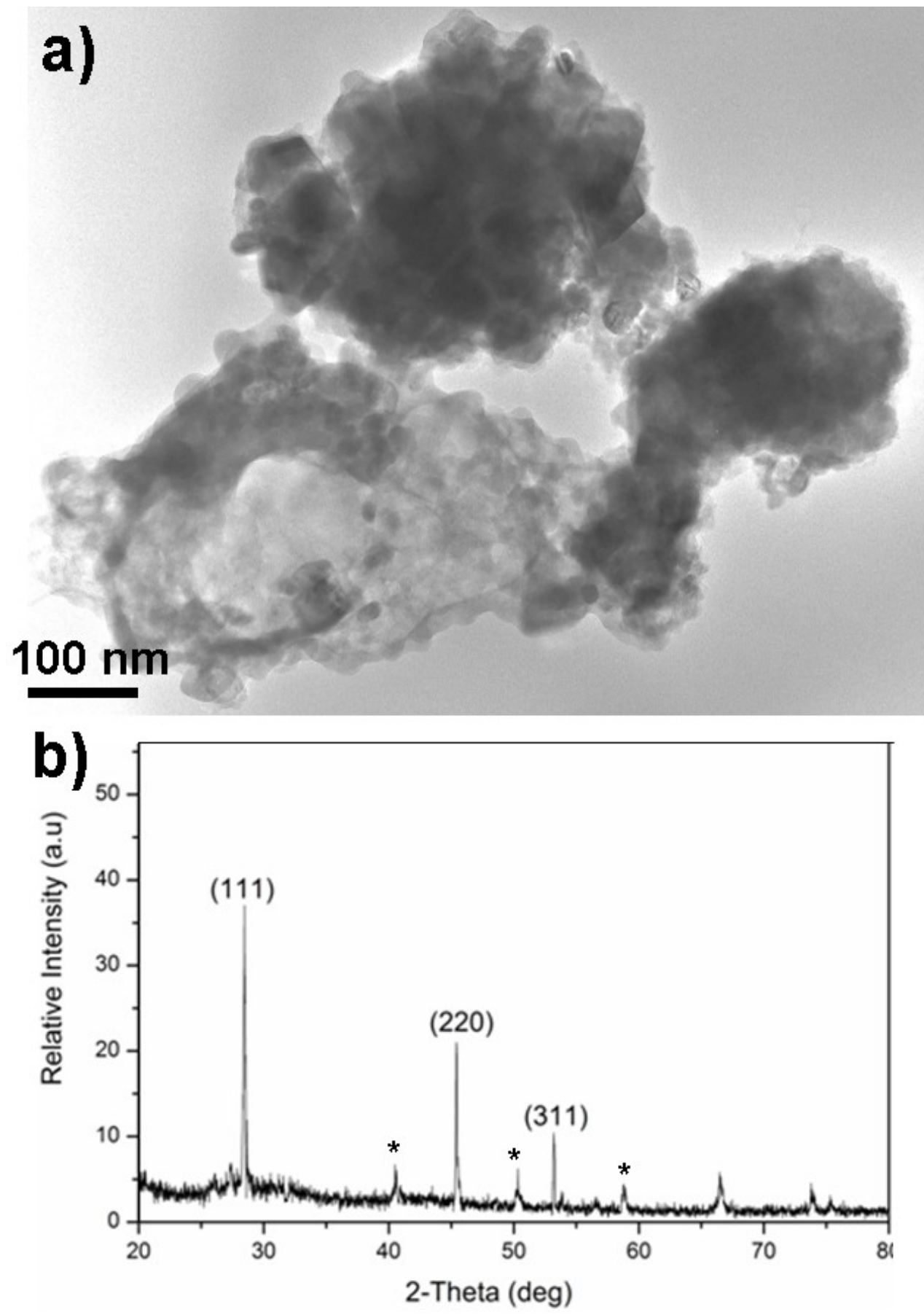

Figure 4.8: (a) TEM image of bulk crystalline germanium and (b) corresponding XRD pattern 
In experiment 7 whilst using surfactant/solvent system (1-octadecene/oleic acid) crystalline nanoparticles of a large size range $(5-25 \mathrm{~nm})$ were synthesized. SAED analysis of the nanoparticles gave the characteristic diamond crystal structure of germanium. Though the addition of oleic acid as a surfactant provided the impetus for size reduction, the large size range was still a disadvantage due to the absence of photoluminescence. Figure $4.9 \mathrm{a}$ is the TEM image of the as-synthesized nanoparticles obtained by the reaction. Figure $4.9 \mathrm{~b}$ is the EDX pattern of the particles shown in figure $4.10 \mathrm{a}$ and shows that germanium is the predominant product with traces of $\mathrm{NaCl}$ as impurities. The Si peak is an artifact from the TEM. 

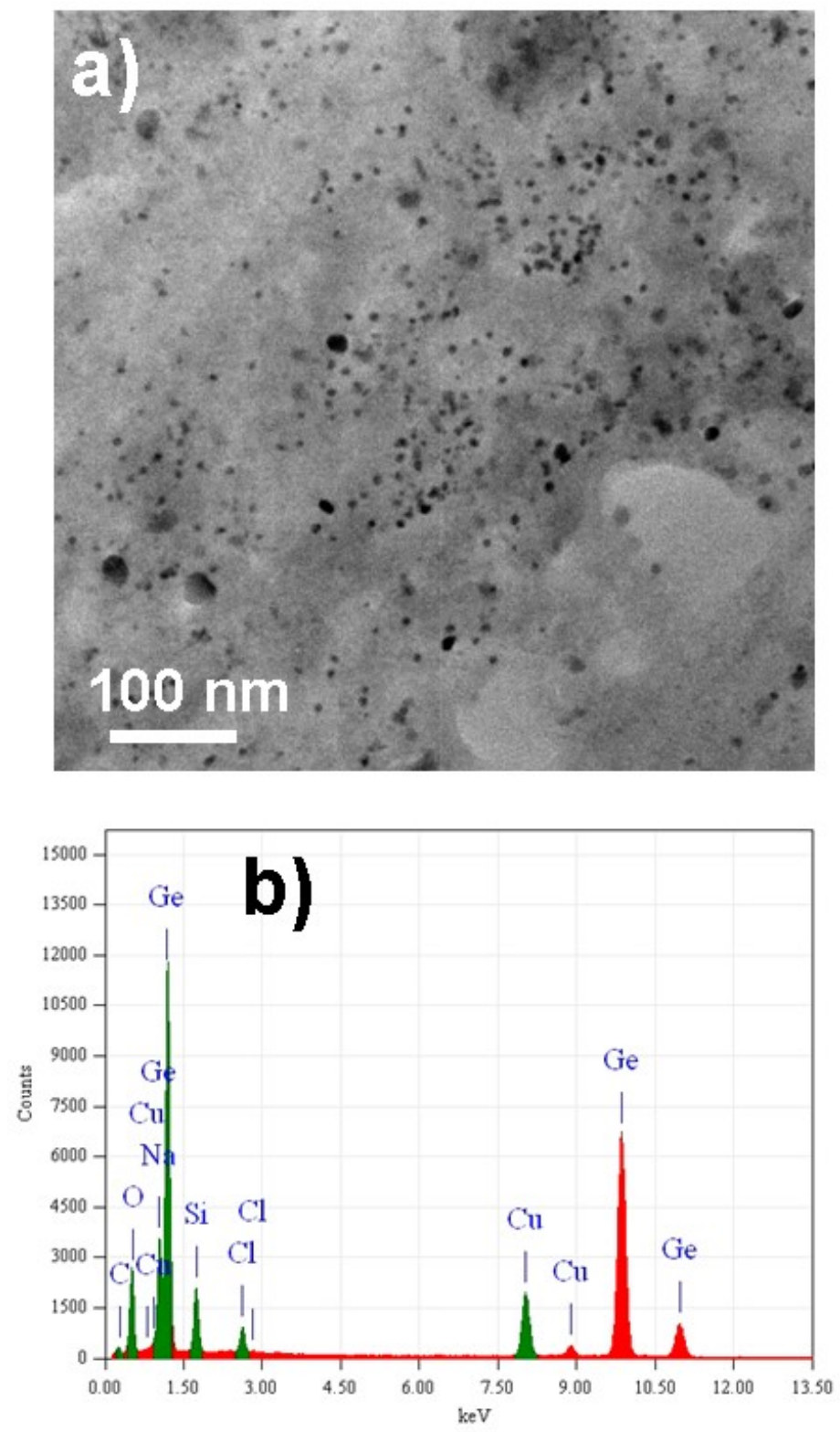

Figure 4.9: (a) TEM image of germanium nanoparticles (b) corresponding EDX pattern 


\subsubsection{Reactions of Germanium Tetrachloride and Sodium Borohydride in oleylamine and oleylamine/oleic acid at High Temperatures (Expt. 8 and 9)}

Experiment 8 and 9 deals with the synthesis of germanium nanocrystals using oleylamine and oleylamine/oleic acid as solvent/surfactant systems. Since the previous experiment failed to produce monodisperse nanocrystals, a solvent system involving a ligand was used.
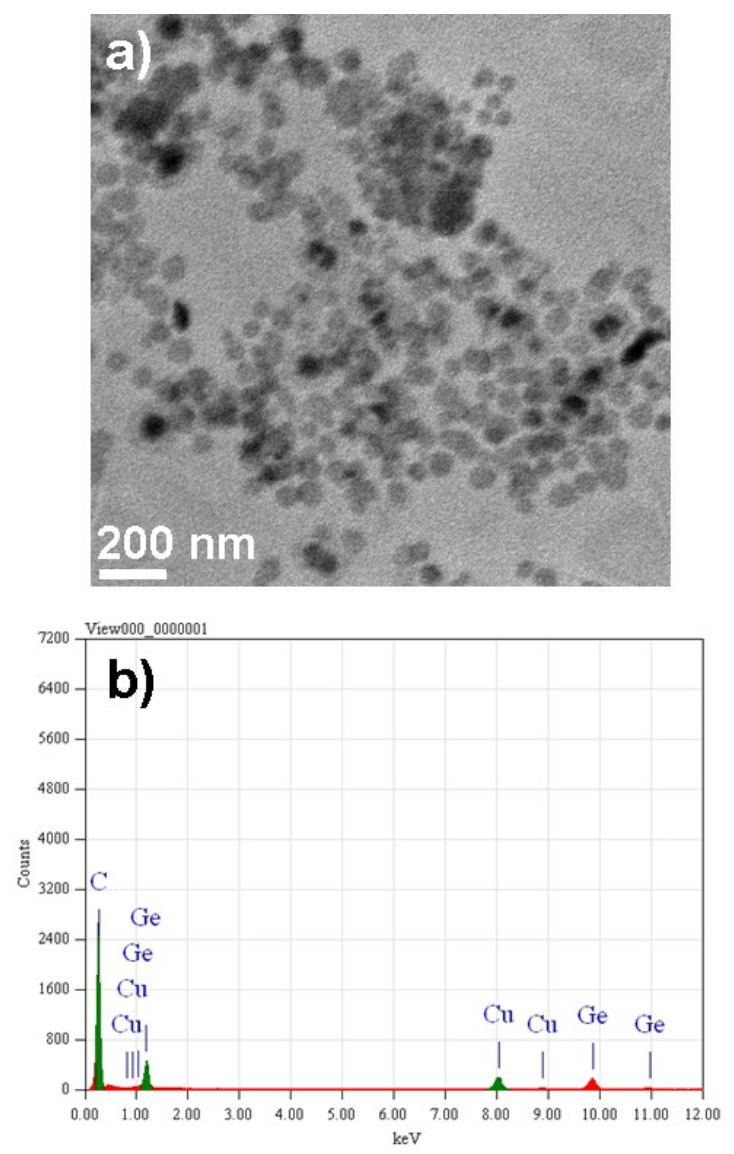

Figure 4.10: (a) TEM image of germanium nanoparticles synthesized using oleylamine as solvent and surfactant (b) corresponding EDX pattern 
Sodium borohydride $\left(\mathrm{NaBH}_{4}\right)$ in triglyme was injected into a degassed solution of either oleylamine or oleylamine/oleic acid mixture and heated up to $200^{\circ} \mathrm{C} . \mathrm{GeCl}_{4}$ is dissolved in either of the two solvent systems in a $\mathrm{N}_{2}$ filled glove box and transferred into an airtight syringe, which is then injected into the above mixture and allowed to react for 10 minutes before the reaction mixture is gradually heated to $300^{\circ} \mathrm{C}$ and refluxed at that temperature for $3 \mathrm{~h}$. On injection the colour of the reaction mixture turns reddish brown and becomes dark brown on reacting after $3 \mathrm{~h}$. The resulting brown powder was washed with excess toluene and ethanol and centrifuged to remove by-products. Figure 4.10a is the TEM image of the as-synthesized product obtained for experiment 8. Amorphous germanium nanoparticles of size range 70-100 nm were synthesized. A large amount of carbonaceous matter from the surfactant prevented the high resolution imaging of the nanoparticles. An EDX pattern of the nanoparticles (figure 4.10b) shows that they are made up of germanium.

Experiment 9 involves the synthesis of germanium nanocrystals using oleylamine/oleic acid as a solvent/surfactant system. The experimental procedures were the same as the previous experiment. The experiment again failed to produce nanocrystals of uniform size distribution. Germanium nanocrystals of size range $4-25 \mathrm{~nm}$ were synthesized. Figure 4.11 is the TEM image of the as-synthesized nanocrystals with the inset being the corresponding selected area diffraction pattern. The nanocrystals were highly crystalline and the SAED pattern corresponded to the characteristic diamond crystal structure of germanium. 


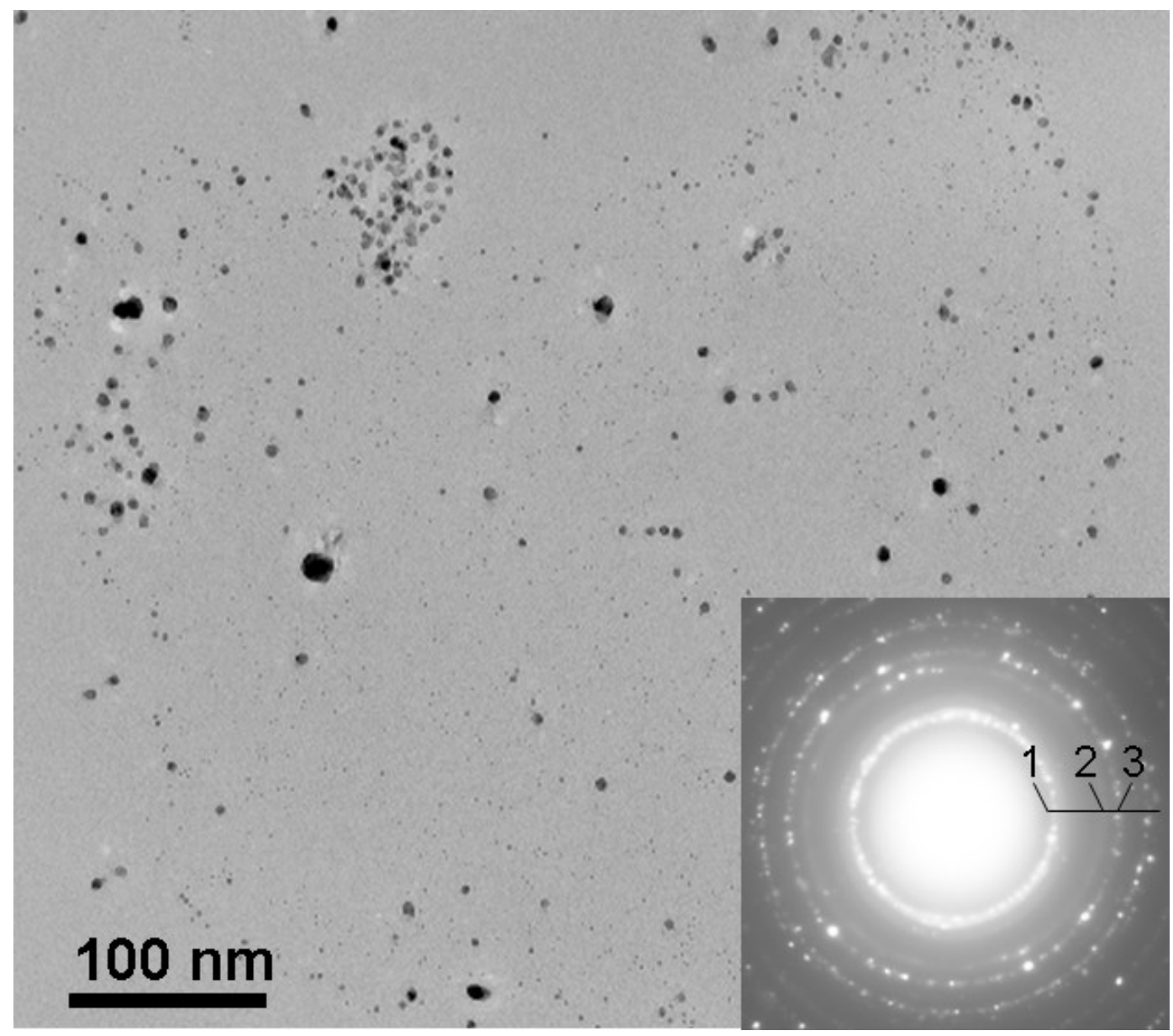

Figure 4.11: TEM image of germanium nanocrystal synthesized using oleylamine oleic acid as surfactant/solvent system (inset) corresponding SAED pattern, with rings matching the diamond germanium planes: Ring $1=(111), 2=(220)$ and $3=(311)$. 


\subsection{Biological Applications *}

(*Note: Both biological imaging and toxicity studies on the germanium nanocrystals were conducted by Dr Kenji Yamamoto and co-workers on the samples. The results described below were not done by the author and are only present to illustrate the applications of the as-synthesized nanocrystals for biological applications.)

One application of germanium nanocrystals is as imaging agents for cell biology and this application is demonstrated with HepG2 cells. Figure 4.7 shows the overlay of the transmission and fluorescence image obtained from HepG2 cells with Ge nanocrystals transfected into the cytosol. The blue fluorescence from direct-bandgap emission from the Ge nanocrystals in the HepG2 cells illustrates the use of hydrophilic germanium nanocrystals as biological fluorescence imaging agents.

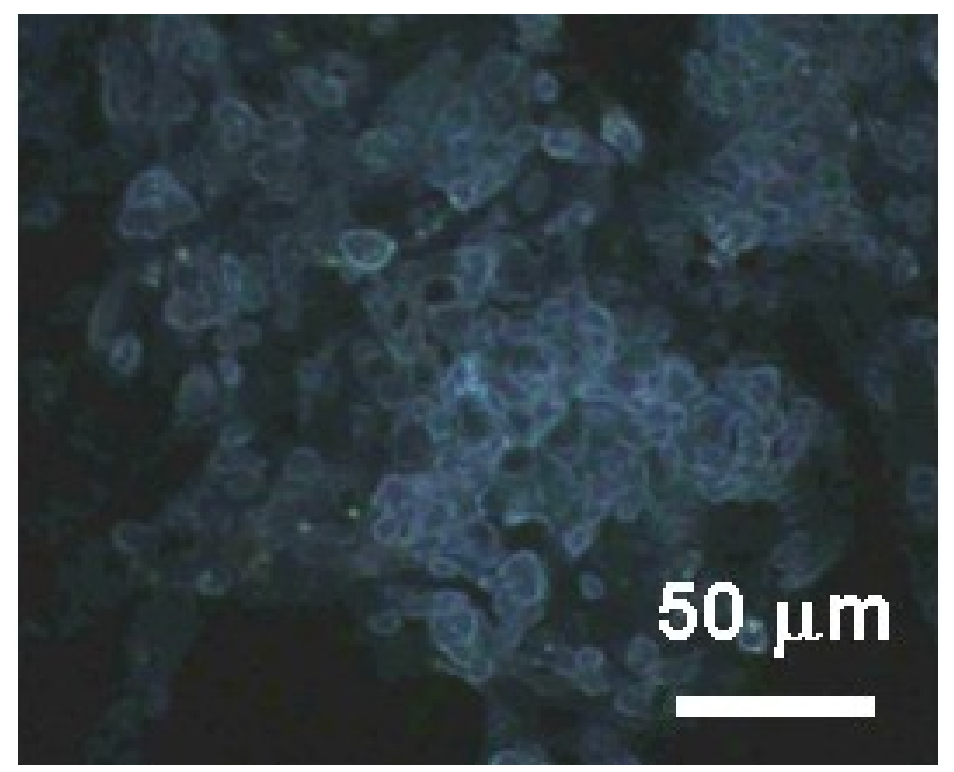

Figure 4.12: Overlay of the transmission and fluorescence microscopy image of HepG2 cells with germanium nanocrystals transfected inside the cytosol. 
To use germanium nanocrystals in biological imaging the effect of cell activity should be known. The mitochondrial activity in $0-1000 \mu \mathrm{g} / \mathrm{ml}$ of germanium nanocrystals was investigated with HePG2 cells as a carcinoma cell line. From the histogram in Figure 4.13, the $50 \%$-inhibitory toxicity concentration (TC50) with HePG2 cells was calculated and found to be $100 \mu \mathrm{g} / \mathrm{mL}$. This indicates that germanium nanocrystals could be used at concentrations of $100 \mu \mathrm{g} / \mathrm{mL}$, and has relatively low toxicity. ${ }^{17}$ A TC 50 of 100 $\mu \mathrm{g} / \mathrm{mL}$ is lower than that reported by Fujioka and co-workers for CdSe quantum dots exposed to UV light with HeLa cells. This present study can provide the basis for further advances on the cytotoxicity of germanium nanoparticles.

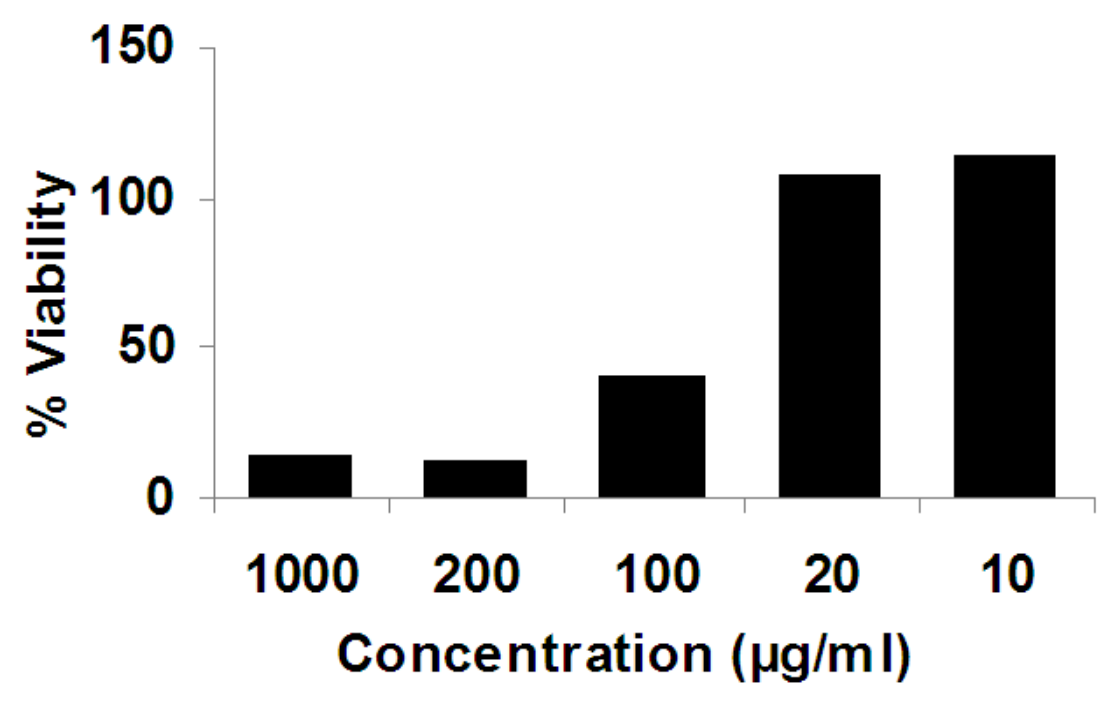

Figure 4.13: Toxicity studies: Mitochondrial activity of HePG2 cells cultured with germanium nanocrystals. 


\subsection{Discussion}

While studying the effect of reducing agents on particles size, it was found that the reaction with $\mathrm{LiAlH}_{4}$ produced the smallest nanocrystals $(4 \pm 0.5 \mathrm{~nm})$ as shown in figure 4.1a. The reaction using $\mathrm{LiAlH}_{4}$ as the reducing agent proceeds vigorously and quickly due to the strong reducing capability of $\mathrm{LiAlH}_{4}$. As a result of this rapid reduction there is a quick depletion of the monomers with few monomers remaining for further growth of the nanocrystals, hence, the small particle size. A similar trend is observed for the reduction by $\mathrm{Li}(\mathrm{Et})_{3} \mathrm{BH}$ and $\mathrm{LiBH}_{4}$, but due to their lower reactivity compared to $\mathrm{LiAlH}_{4}$, slightly larger nanoparticle sizes are obtained. $\mathrm{NaBH}_{4}$ is a weak reducing agent, and when injected into the solution there is less monomer depletion in the nucleation burst leaving considerable unreacted monomer that contributes to nanoparticle growth, thus causing the formation of nanoparticles of large size.

The ability to control particle size in group IV nanocrystals using reducing agents is uncommon and not reported for systems involving hydride reducing agents and inverse micelles. Of particular significance is the ability to control nanoparticle size in the quantum confinement regime.

Germanium nanocrystals of different sizes were obtained while studying the effect of precursor concentration on particle size. According to La Mer and Dinegar's nucleation theory, ${ }^{18}$ the precursor concentration is an important factor that controls nanoparticle size. At lower concentrations $(0.46 \mathrm{mmol})$ the smallest particle sizes 
were obtained, whilst at higher concentrations particles of large sizes were obtained (1.39 mmol). This is consistent with the theory that, a higher percentage of nonuniform growth and polydispersion will be observed when the initial solutions are concentrated. For the concentrated sample in this study (Figure 4.4), the rate of Ge seed production becomes high, thus producing a large nanocrystal size.

The high temperature reactions involving the reduction of germanium tetracholride at $200^{\circ}$ by sodium borohydride, followed by the crystallization at $300^{\circ}$ using 1 octadecene,1-octadecene/oleic acid, oleylamine and oleylamine/oleic acid failed to produce monodisperse germanium nanocrystals of uniform size distribution. Experiment 6 produced bulk germanium, mainly due to the non-coordinating ability of 1-octadecene, resulting in its failure to bind to the germanium during nucleation causing it to nucleate into bulk materials. Experiment 7 which involved the addition of oleic acid to aid in the formation of nanocrystals, though succesful in forming crystalline nanoparticles failed to produce an uniform size distribution. Size selective purifcation could aid in separation of the nanocrystals from the larger particles rendering them useful for applications.

Similarly experiment 8 which involved the use of oleylamine as surfacatant and solvent failed to produce monodisperse germanium nanocrystals. Amorphous germanium nanoparticles of large sizes were obtained. The failure of oleylamine to bind to bind to the particles during nucleation could be a reas on for its large particle size and the lack of crystallization. Experiment 9 however produced nanocrystals of 
size range 4-25 $\mathrm{nm}$. Size selective purifcation could again aid in separation of the nanocrystals from the larger particles rendering them useful for applications. It could be said that the addition of oleic acid to the reaction mixture has faciliated, atleast in part the formation of germanium nanocrystals by high temperature reduction routes. 


\subsection{Summary}

In conclusion germanium nanocrystals with narrow size distributions were synthesized in reverse micelles and powerful reducing agents at room temperature and pressure. By using the difference in reactivity of the reducing agents, size control of the quantum dot

was achieved. It was observed that by using $\mathrm{LiAlH}_{4}$, the strongest reductant, small nanoparticles could be obtained and by using weaker reductants such as $\mathrm{NaBH}_{4}$, larger nanoparticles were obtained. In addition it was found that by varying the concentration of the precursors, particle sizes could be controlled. The surface of the germanium nanocrystals were made water-soluble by capping with allylamine. The germanium nanocrystals were purified by column chromatography. The germanium nanocrystals dispersed in aqueous solvents displayed strong photoluminescence in the blue region of the visible spectrum. The amine functionalized germanium nanocrystals were used for the imaging of HePG2 cells and were shown to be relatively non-toxic. The optical properties, purity and cell compatibility of the germanium nanocrystals reported here make them excellent candidates for future biological optical probes. High temperature reactions involving a high temperature reduction where also studied. It was found that the addition of oleic acid to the reaction mixture facilitated particle size control. 


\subsection{Experimental}

The Microemulsion Synthesis of Germanium Nanocrystals: In a typical synthesis, 50 $\mathrm{ml}$ of anhydrous hexane (Aldrich, $\geq 99 \%$ ) was added to a 3-necked flask attached to a schlenk line, and purged with nitrogen. $0.026 \mathrm{~g}$ of Pentaethylene glycol monododecyl ether $\left(\mathrm{C}_{12} \mathrm{E}_{5}\right)$ a non-ionic surfactant (Nikko Chemicals Co, $>98 \%$ ) and $100 \mu \mathrm{L}$ of $\mathrm{GeCl}_{4}$ (Aldrich, $\geq 99.0 \%$ ) was removed from the glove-box in an air-tight syringes and injected into the reaction flask. Germanium nanocrystals were then formed by adding a stoichiometric amount $\left(1: 4, \mathrm{GeCl}_{4}\right.$ : reductant $)$ of a solution of either $\mathrm{LiAlH}_{4}(1.0 \mathrm{M}$ in THF), $\mathrm{Li}(\mathrm{Et})_{3} \mathrm{BH}\left(1.0 \mathrm{M}\right.$ in THF), $\mathrm{LiBH}_{4}\left(2.0 \mathrm{M}\right.$ in THF) or $\mathrm{NaBH}_{4}(2.0 \mathrm{M}$ in triethylene glycol dimethyl ether) as reducing agent and were all purchased from Aldrich Chemicals. After the above mixture was left to react for 2 hours, the surface of the nanocrystals is terminated with hydrogen. $40 \mu \mathrm{L}$ of $\mathrm{H}_{2} \mathrm{PtCl}_{6}(99.995 \%$, Aldrich) in anhydrous isopropanol $(99.5 \%$, Aldrich) as catalyst and $2 \mathrm{ml}$ of allylamine $(\geq 99 \%$, Aldrich) were added to modify the surface germanium-hydrogen bonds thus rendering the nanocrystals water-soluble.

Separation and Purification: The water-soluble Ge nanocrystals were purified by first removing the solvent by rotary evaporation to produce yellow viscous oil. $50 \mathrm{ml}$ of distilled water was then added to the flask, in which the nanocrystals dissolved but not the $\mathrm{C}_{12} \mathrm{E}_{5}$ which is subsequently removed by filtration. After sonication for 5 minutes the solution was further filtered using a Millipore $0.45 \mu \mathrm{m}$ membrane filter. The filtrate was concentrated to about $1 \mathrm{ml}$ and then purified down a Sephadex gel LH-20 (bead size 
$25-100 \mu \mathrm{m}$ a column $(\varphi=1 \mathrm{~cm})$. The luminescent fraction was then collected and concentrated down to $1 \mathrm{~mL}$ to give a solution of water-soluble germanium nanocrystals.

Incorporation of germanium nanocrystals into HepG2 cells: HepG2 cells were used with assays conducted at $1.0 \mu \mathrm{g} / \mathrm{mL}$ for 6 hours incubation. The germanium nanocrystals were excited with a mercury lamp, and the images were captured on a cooled chargecoupled device (CCD), mounted on a fluorescent microscope IX-81 (Olympus) with an excitation filter $330-380 \mathrm{~nm}$.

High Temperature Synthesis of Germanium Nanocrystals: Sodium borohydride in Triglyme $(2 \mathrm{ml}, 2 \mathrm{M})$ was added to either 1-octadecene $(4 \mathrm{ml})$ or 1octadecene/oleylamine $(4 \mathrm{ml} / 2.5 \mathrm{ml})$ or oleylamine $(4 \mathrm{ml})$ or oleylamine/oleic acid $(4 \mathrm{ml} / 2.5 \mathrm{ml})$ and stirred under $\mathrm{N}_{2}$ in a three necked flask. The mixture is gradually heated to $200^{\circ} \mathrm{C} .0 .1 \mathrm{ml}(0.46 \mathrm{mmol})$ of germanium tetrachloride was mixed with the respective solvent/surfactant systems in the same quantity as above in a $\mathrm{N}_{2}$ filled glovebox and taken in air-tight gas syringe and injected into the reaction mixture. The reaction mixture is then raised rapidly to $300^{\circ} \mathrm{C}$ and reacted at that temperature for $3 \mathrm{~h}$.

Purification: The as-synthesized materials is first washed and centrifuged in toluene to remove solvents and then repeatedly washed and centrifuged in methanol to remove impurities. The final dry powder is then dispersed in hexane for analysis. 


\subsection{References}

1. Fok, E.; Shih, M.; Meldrum, A.; Veinot, J. C., Preparation of alkyl-surface functionalized germanium quantum dots via thermally initiated hydrogermylation, Chem. Commun. 2004, 386-387.

2. Chiu, H. W.; Kauzlarich, S. M., Investigations of reaction conditions for optimal germanium nanoparticle production by a simple reduction route, Chem. Mater. 2006, 18, 1023-1028.

3. Lu, X.; Korgel, B. A.; Johnston, K. P., High yield of germanium nanocrystals synthesized from germanium diiodide in solution, Chem. Mater. 2005, 17, 64796485.

4. Lu, X.; Ziegler, K. J.; Ghezelbash, A.; Johnston, K. P.; Korgel, B. A., Synthesis of Germanium Nanocrystals in High Temperature Supercritical Fluid Solvents, Nano Lett. 2004, 4, 969.

5. Myung, N.; Lu, X.; Johnston, K. P.; Bard, A. J., Electrogenerated chemiluminescence of Ge Nanocrystals, Nano Lett. 2004, 4, 183. 
6. Lu, X.; Korgel, B. A.; Johnston, K. P., Synthesis of Germanium Nanocrystals in High Temperature Supercritical Fluid Solvents, Nanotechnology 2005, 16, 389.

7. Wu, H. P.; Liu, J. F.; Wang, Y. W.; Zeng, Y. W.; Wu, J., Preparation of Ge nanocrystals via ultrasonic solution reduction, J. Mater. Lett. 2006, 60, 986.

8. Wilcoxon, J. P.; Provencio, P. P.; Samara, G. A., Synthesis and optical properties of germanium nanocrystals, Phys. Rev. B 2001, 64, 035417.

9. Wilcoxon, J. P.; Provencio, P. P.; Samara, G. A., Optical and electronic properties of Si nanoclusters synthesized in inverse micelles, Phys. Rev. B 1999, 60, 2704.

10. Lambert, T. N.; Andrews, N. L.; Gerung, H.; Boyle, T. J.; Oliver, J. M,; Wilson, B. S.; Han, S. M., Water-soluble germanium (0) nanocrystals: cell recognition and near-infra red photothermal conversion properties, Small 2007, 3(4) 691-699.

11. Gerung, H.; Bunge, S. D.; Boyle, T. J.; Brinker, C. J.; Han, S. M., Anhydrous solution synthesis of germanium nanocrystals from the germanium(II) precursor $\mathrm{Ge}\left[\mathrm{N}\left(\mathrm{SiMe}_{3}\right)_{2}\right]_{2}$, Chem. Comm. 2005, 1914-1916. 
12. Al-Salim, N.; Young, A. G.; Tilley, R. D.; McQuillan, A. J.; Xia, J., Synthesis of CdSeS nanocrystals in coordinating and noncoordinating solvents: solvent's role in evolution of the optical and structural properties, Chem. Mater. 2007, 19, $5185-5193$.

13. Warner, J. H.; Rubinsztein-Dunlop, H.; Tilley, R. D., Surface morphology dependent photoluminescence from colloidal silicon nanocrystals, J. Phys. Chem B. 2005, 109, 19064-19067

14. Taylor, B. R.; Kauzlarich, S. M.; Delgado, G. R.; Lee, H. W. H., Synthesis and characterization of germanium/Si-alkyl and germanium/silica core-shell quantum dots, Chem. Mater. 1999, 11, 2493.

15. Henderson, E. J.; Hessel, C. M.; Veinot, J. G. C., Synthesis and photoluminescent properties of size controlled germanium nanocrystals from phenyl trichlorogermane-derived polymers, J. Am. Chem. Soc. 2008, 130, 3624.

16. Ma, X.; Wu, F.; Kauzlarich, S. M., Alkyl-terminated crystalline Ge nanoparticles prepared from $\mathrm{NaGe}$ : Synthesis and functionalization and optical properties, J. Solid State Chem. 2008, 181, 1628. 
17. Fujioka, K.; Hiruoka, M.; Sato, K.; Manabe, N.; Miyasaka, R.; Hanada, S.; Hoshino, A.; Tilley, R. D.; Manome, Y.; Hirakuri, K.; Yamamoto, K., Luminescent passive-oxidized silicon quantum dots as biological staining labels and their cytotoxicity effects at high concentration, Nanotechnology, 2008, 19, 415102 .

18. La Mer, V. L; Dinegar, R. H., Theory, production, and mechanism of formation of monodispersed hydrosols, J. Am. Chem. Soc. 1950 72, 4847-4854. 


\section{Inorganic Fluorescent Whitening Agents}

\subsection{Introduction}

This chapter describes the application of colloidal silicon and germanium nanocrystals as fluorescent whitening agents in wool fabrics. The studies detailed in this chapter have been done in collaboration with Dr Santanu Deb Choudhury and Dr Jolon Dyer of AgResearch, Christchurch.

The yellowing of wool garments in sunlight impedes the marketability of wool fabrics, especially where light and pastel shades are desirable. FWAs, also called optical brightening agents (OBAs), are used as additives to brighten colours and mask natural yellowing. FWAs convert ultraviolet light into longer wavelength visible light on the blue region of the spectrum, causing treated fabric to reflect a greater proportion of visible light and making it appear brighter. Since the tone of the extra light reflected is on the blue side of the spectrum, this blue-violet tinge will counter any yellowing on the fabric to make it look whiter, as well as brighter. When fixed in solid substrates, brighteners fluoresce in the blue. ${ }^{1}$ Organic based fluorescent whitening agents (FWAs) have been utilized to improve the initial whiteness of wool fabrics by absorbing UV light and emitting visible blue light, thus masking this yellowing. ${ }^{2-5}$ However, this increase in whiteness is brief, as the addition of FWAs exacerbates the yellowing process relative to untreated wool and leads to severe photoyellowing. ${ }^{2}$ This has been shown by previous studies to be due to the production of reactive oxygen species (ROS), produced by wool

keratin and FWA's when irradiated with UV wavelengths in air. ${ }^{5}$ Further studies have 
shown that it is the subsequent attack of ROS upon certain amino acid residues present in wool, attack of ROS on the FWA itself, or a combination of both of these processes that leads to the formation of yellow chromophores in FWA-treated wool. ${ }^{6}$

The motivation for this research came from the fact that both Si and Ge nanocrystals emit blue light under UV radiation and are inert. ${ }^{7-9}$ The silicon and germanium nanocrystals were produced using the micro-emulsion synthesis detailed in chapters 3 and 4 , and were functionalized using allylamine and 1-hexene as capping agents. The nanocrystals were incorporated into the wool fabric by means of a silica based sol-gel matrix, coated onto its surface. Both amine and 1-hexene terminated silicon and germanium nanocrystals were dispersed into this matrix.

\begin{tabular}{|c|c|}
\hline S.No & Fluorescent Whitening Agent \\
\hline 1. & 1-Hexene capped silicon nanocrystals \\
\hline 2. & Allylamine capped silicon nanocrystals \\
\hline 3. & 1-Hexene capped germanium nanocrystals \\
\hline 4. & Allylamine capped germanium nanocrystals \\
\hline 5. & Uvitex (commercial organic FWA) \\
\hline
\end{tabular}

Table 5.1: List of fluorescent whitening agents used in this study

To test the ability of silicon and germanium nanocrystals as an alternate for commercial organic FWA's, the following samples (Table 5.1) were prepared and coated onto the surface of the wool. Silica sol-gel matrices treatments were used along with the whitening agents. In addition to the above samples, blank samples containing only sol-gel coating and without any coating were also prepared. After coating the wool fabrics with 
FWA's, they were subject to various tests. Colour measurements to determine the level of yellowness and photodegradation were conducted after irradiation of the fabric with UVA and UVB lamps. Since reactive oxygen species (ROS) are responsible for the yellowing of wool fabric, an HPLC assay was conducted to determine their generation by FWA's. Electron microscopy studies of the fabric were conducted to provide insight into the structure and morphology of the fibres before and after coating. Fluorescence spectroscopy studies were conducted on the wool fibres and the nanocrystal containing sol-gel matrix to study the luminescence. Finally to test the commercial viability of the modified wool fabrics, their durability after several wash and abrasion tests were conducted. 


\subsection{Fabrication of Inorganic Fluorescent Whitening Agents}

\subsubsection{Synthesis of Silicon and Germanium Nanocrystals}

Silicon and germanium nanocrystals of size, approximately $2.5 \pm 0.5 \mathrm{~nm}$ and $5 \pm 0.5 \mathrm{~nm}$ respectively were synthesized using techniques detailed in chapters 3 and 4 of this thesis. Solutions containing either low concentration $\left(\sim 2.106 \times 10^{15}\right.$ particles $\left./ \mathrm{ml}\right)$ or high concentration $\left(\sim 6.318 \times 10^{15}\right.$ particles $\left./ \mathrm{ml}\right)$ were prepared. The nanocrystals were either surface modified with allylamine or 1-hexene and exhibited strong blue photoluminescence.

\subsubsection{Preparation of nanocrystal incorporated sol-gel coated wool fabric}

A silica based sol-gel matrix using tetraethyl orthosilicate was prepared at room temperature. To facilitate a thin distribution of the matrix onto the wool fabric, a diluent containing the organic solvent, di(propylene glycol) n-propyl ether (DPnP) was used in combination with water. The high water to $\mathrm{DPnP}$ ratio $(75 \%$ water, $25 \% \mathrm{DPnP})$ also meant that this application was environmentally friendly and commercially viable. To the above mixture colloidal solutions of silicon and germanium nanocrystals, including the commercial whitening agent Uvitex were injected and stirred for an hour. Uvitex is a commercially available, stilbene based fluorescent whitening agent. 


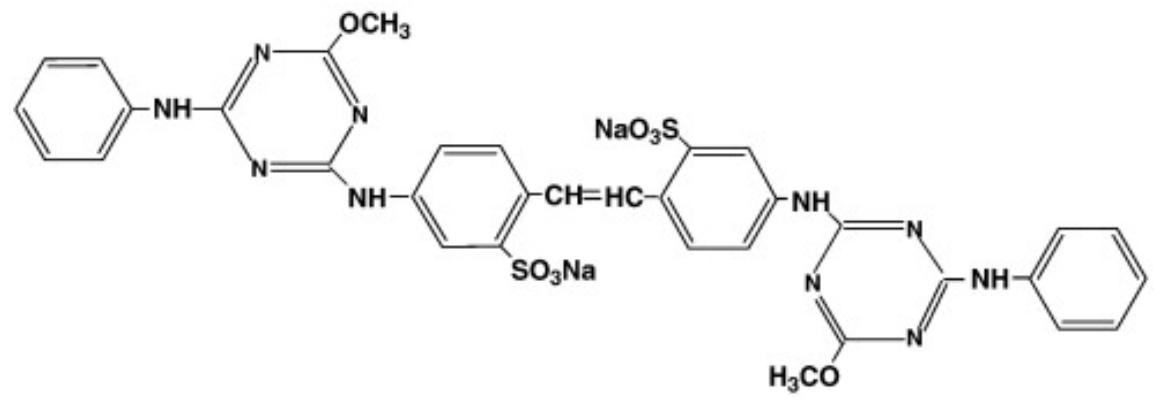

Figure 5.1: Chemical structure of Uvitex

Wool Fabric samples $(6 \times 6 \mathrm{~cm})$ were dipped into this mixture and allowed to dry overnight at room temperature to form nanocrystal incorporated sol-gel coated wool fabrics. The fabrics were then annealed at $120^{\circ} \mathrm{C}$ for an hour to remove any remaining moisture. 


\subsection{Photoluminescence Studies}

The aim of this study was to determine the effect of the nanocrystals to act as fluorescent whitening agents. Photoluminescence studies of the nanocrystals in solution and the wool fibres with or without the sol-gel/nanocrystal matrix were conducted.

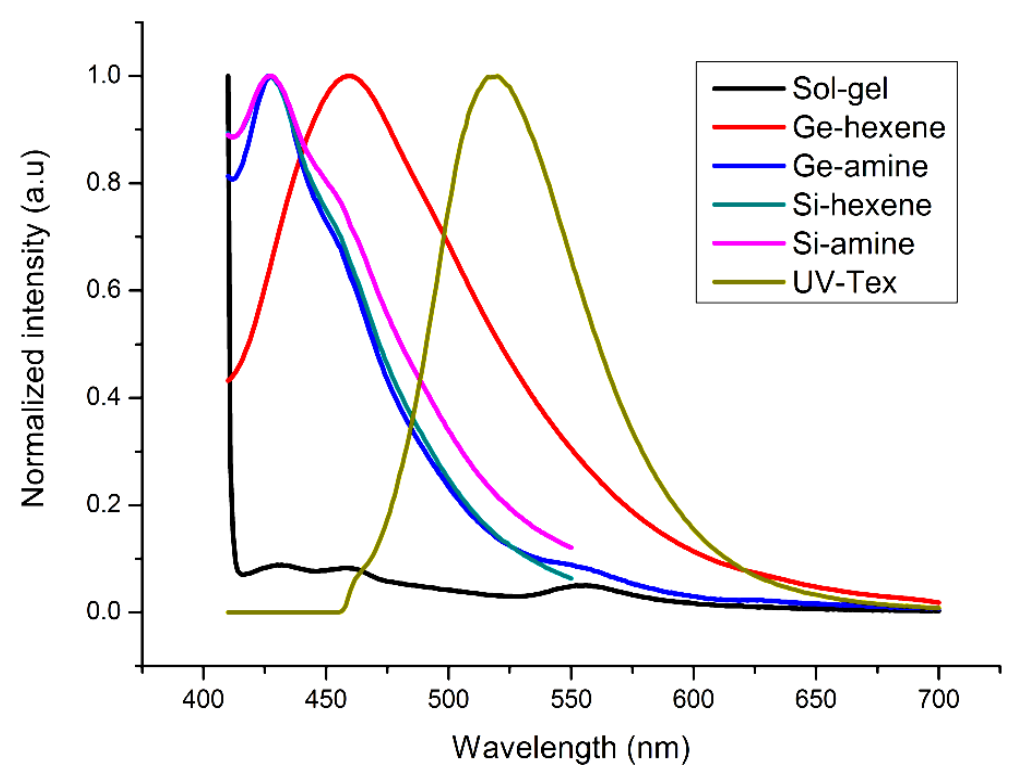

Figure 5.2: Normalized photoluminescence spectrum of nanocrystal incorporated solgel matrices excited at $400 \mathrm{~nm}$.

Figure 5.2 is the photoluminescence spectra of solutions of the fluorescent whitening agents dissolved in sol-gel matrixes. After incorporating the fluorescing whitening agents into the sol-gel matrix, their fluorescence was measured using a spectrofluorometer. The excitations at $400 \mathrm{~nm}$ gave rise to sharp emissions across a relatively narrow region of 425 to 450 for the Si and Ge nanocrystal incorporated sol-gel matrix solutions. However, there was no observed emission from the solution containing just the sol-gel 
matrix (black trace). The commercially available fluorescent whitening agent Uvitex had a slightly red shifted emission compared to the nanocrystals $(530 \mathrm{~nm})$.

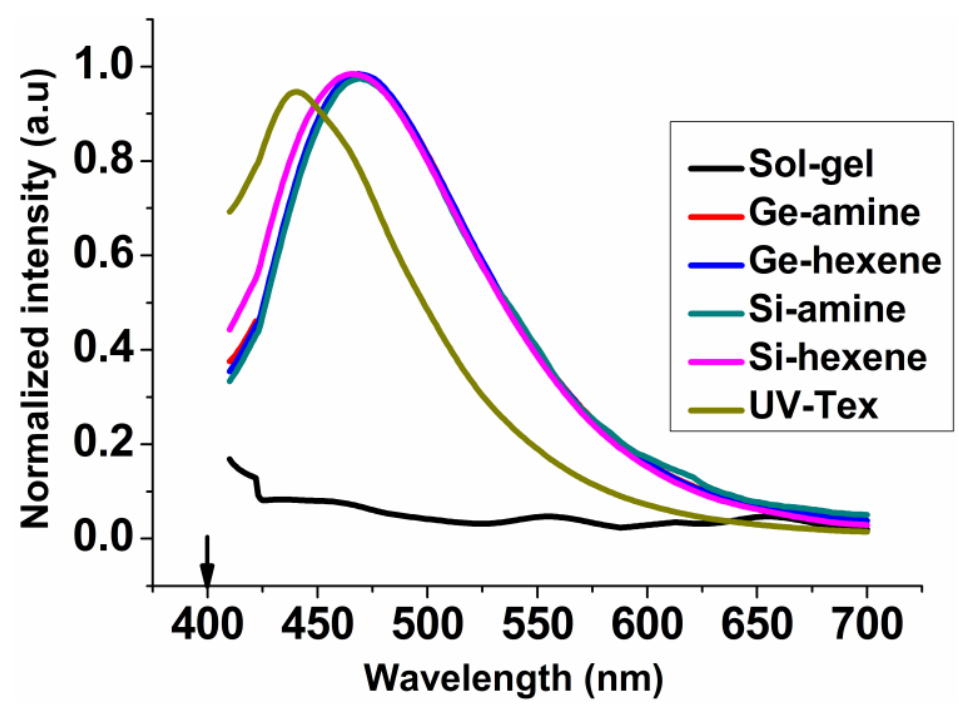

Figure 5.3: Normalized photoluminescence spectrum of wool coated with nanocrystal incorporated sol-gel matrices and excited at $400 \mathrm{~nm}$.

Figure 5.3 is the photoluminescence spectrum of wool samples coated with the fluorescent whitening agents. The samples after coating with fluorescent whitening agents were dried overnight and their fluorescence measured using a spectrofluorometer. The sol-gel coated wool sample still showed no fluorescence. The nanocrystal coated wool samples however appear slightly shifted compared to the measurements done in the solution, and had maximum emissions at $475 \mathrm{~nm}$. The commercial FWA Uvitex has an emission at $430 \mathrm{~nm}$ compared to its emission in the solution $(530 \mathrm{~nm})$. Although it appears that the organic commercial whitening agent (UV-Tex) is brighter compared to the inorganic whitening agents, it is not so. 


\subsection{Photoprotection analysis}

This section details the evaluation, of the degree of yellowing of the various FWA coated wool fabrics. To determine the degree of yellowing in the wool fabrics a chromameter was used. Squares of wool fabric treated with the various fluorescent whitening agents were exposed to UVA and UVB radiation to determine their photoprotective effect. The samples were exposed to UVA radiation $(400 \mathrm{~nm}-320 \mathrm{~nm})$ and UVB radiation $(320 \mathrm{~nm}-280 \mathrm{~nm}$ ) for time periods of $24-48 \mathrm{~h}$ and $12-24 \mathrm{~h}$ respectively. The UVA and UVB dosages obtained from the irradiation chamber used in this study were $52.670 \mathrm{Wm}^{-2}$ and $35.337 \mathrm{Wm}^{-2}$, respectively $\left(\mathrm{Wm}^{-2}=\mathrm{SI}\right.$ unit for solar irradiance) For the UVA irradiation, therefore, a $24 \mathrm{~h}$ exposure is equivalent to approximately 1 to 2 years of regular sunlight exposure through window glass. ${ }^{10}$ Fabric that has been exposed to such radiation may undergo both photobleaching and photoyellowing, due to the formation and subsequent destruction of protein derived chromophores. The photostability of fabrics under these irradiation conditions therefore represents a good correspondence to the colour stability that can be achieved by these treatments in terms of real product life.

In order to determine the photoyellowing effect, the CIE Y-Z values were recorded. A higher Y-Z value indicates an increase in yellowness of the sample, while a lower Y-Z value indicates improved whiteness. $\triangle \mathrm{CIE} \mathrm{Y}-\mathrm{Z}$ fabric scores (sample $\mathrm{Y}-\mathrm{Z}$ minus control $\mathrm{Y}-\mathrm{Z}$ ) were tabulated and plotted to demonstrate the change in yellowness after treatment before and after UVA and UVB radiation. (A detailed discussion on the CIE 
colour space and its relevance to the current study has been given in section 1.7.1 of chapter 1). Each colour measurement represents the average of nine readings from three separate samples. A high concentration of nanoparticles $\left(6.318 \times 10^{15}\right.$ particles $\left./ \mathrm{ml}\right)$ and a low concentration $\left(2.106 \times 10^{15}\right.$ particles $\left./ \mathrm{ml}\right)$ were dispersed in the Si-matrix and then coated onto fabric pieces to assess their photo-protective effect. Seven different treatments were applied to the fabric and are listed in table 5.2.

\begin{tabular}{|c|c|}
\hline Treatments & Fluorescent whitening agent \\
\hline 1 (Control) & - \\
\hline 2 (Sol-gel matrix) & Amine capped Ge particles \\
\hline 3 & 1-Hexene capped Ge particles \\
\hline 4 & Amine capped Si particles \\
\hline 5 & 1-Hexene capped Ge particles \\
\hline 6 & Uvitex \\
\hline 7
\end{tabular}

Table 5.2: List of FWA treatments on the wool fabric 


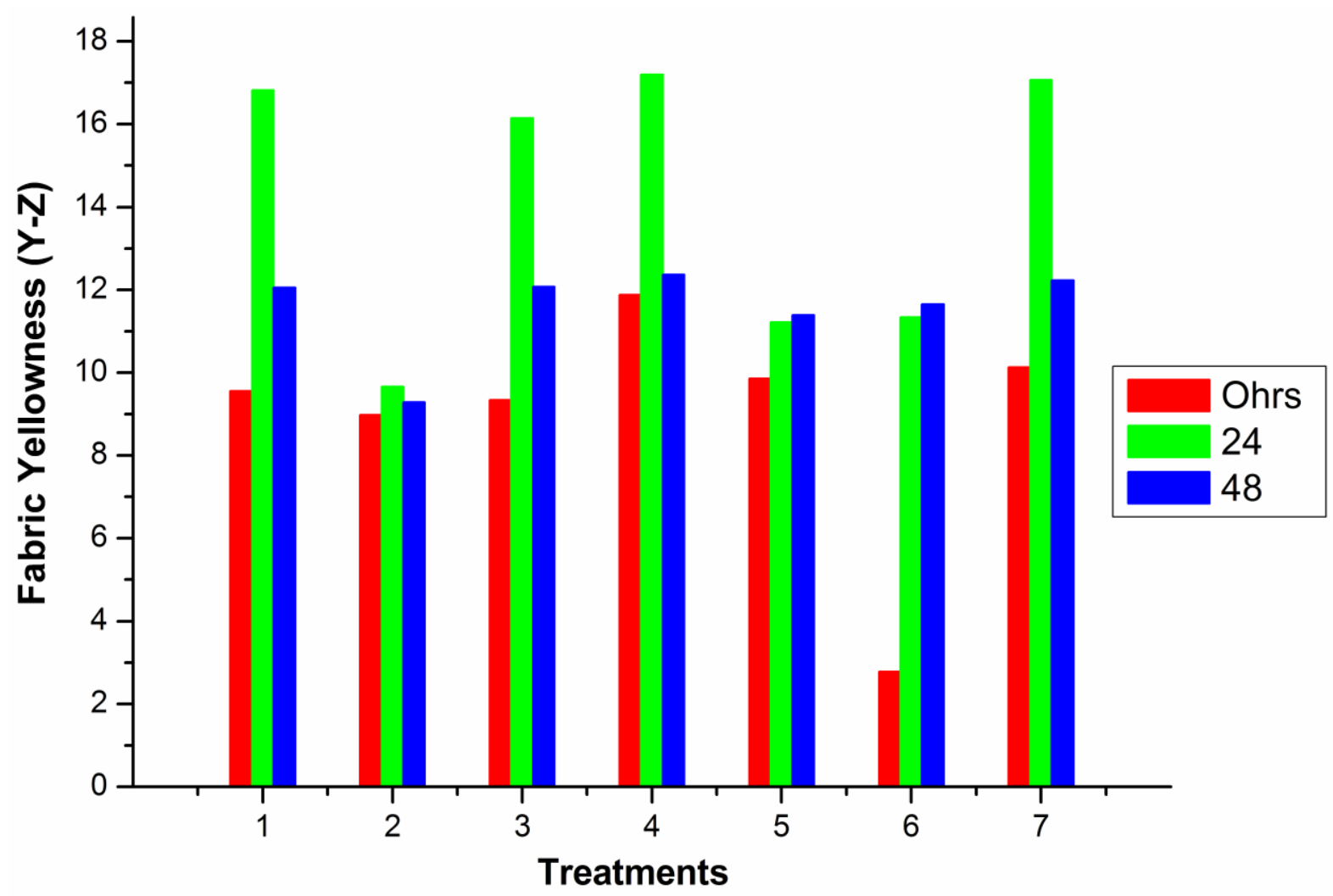

Figure 5.4: CIE Y-Z (yellowness) fabric scores before and after $24 \mathrm{~h}$ and $48 \mathrm{~h}$ UVA irradiation, with low concentration of nanoparticles $(2.106 \times 1015$ particles $/ \mathrm{ml})$ dispersed in the matrix. (1) Control (untreated fabric); (2) Matrix treated; (3) Matrix + Geamine; (4) Matrix + Ge-alkene; (5) Matrix + Si-amine; (6) Matrix + Si-alkene; (7) Matrix + Uvitex.

The $\mathrm{Y}-\mathrm{Z}$ values of fabrics exposed to UVA radiation after coating with Si-matrix and a low concentration of nanoparticles $\left(2.106 \times 10^{15}\right.$ particles $\left./ \mathrm{ml}\right)$ is shown in Figure 5.4. The differences in CIE Y-Z value between the treated fabric and the control are displayed in Figure 5.5. The CIE Y-Z values after UVB radiation are displayed in Figure 5.6 and the differences in CIE Y-Z value in Figure 5.7. 
At $0 \mathrm{~h}$ of exposure to UVA radiation, apart from the 1-hexene capped Si nanocrystals treated fabric, all other treatments had started to yellow considerably. At $24 \mathrm{~h}$ exposure, apart from the matrix, Si-hexene and Si amine coated fabrics; the rest had CIE Y-Z values of about 16-17. After 48h of UVA exposure the yellowness of the fabrics is seen to have decreased to lower values, indicating that the fabrics have undergone photobleaching. However the Si-Hexene capped particles remained stable from photobleaching and only showed a slight increase in photoyellowing.

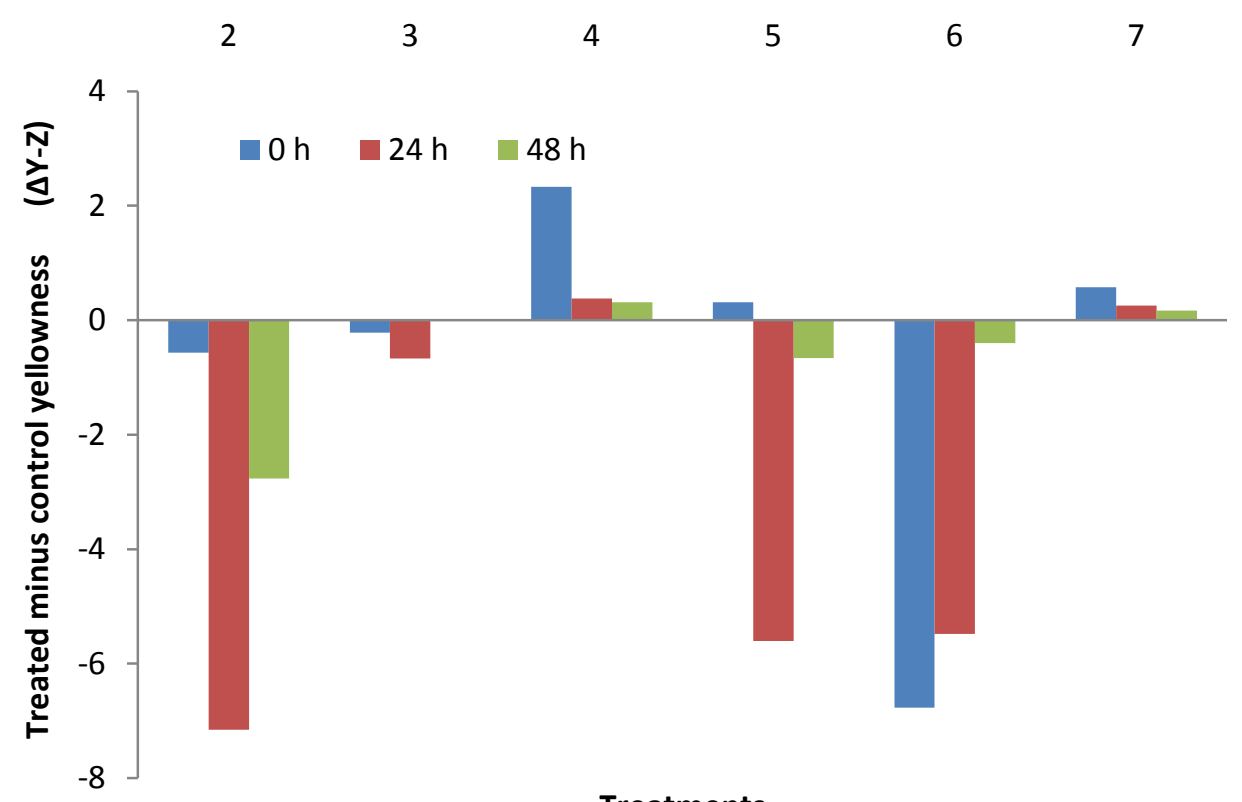

Treatments

Figure 5.5: $\Delta$ CIE Y-Z fabric scores (sample $Y-Z$ minus control $Y-Z$ ) for (2) Matrix treated; (3) Matrix + Ge-amine; (4) Matrix + Ge-alkene; (5) Matrix + Si-amine; (6) Matrix + Si-alkene; (7) Matrix + Uvitex. 


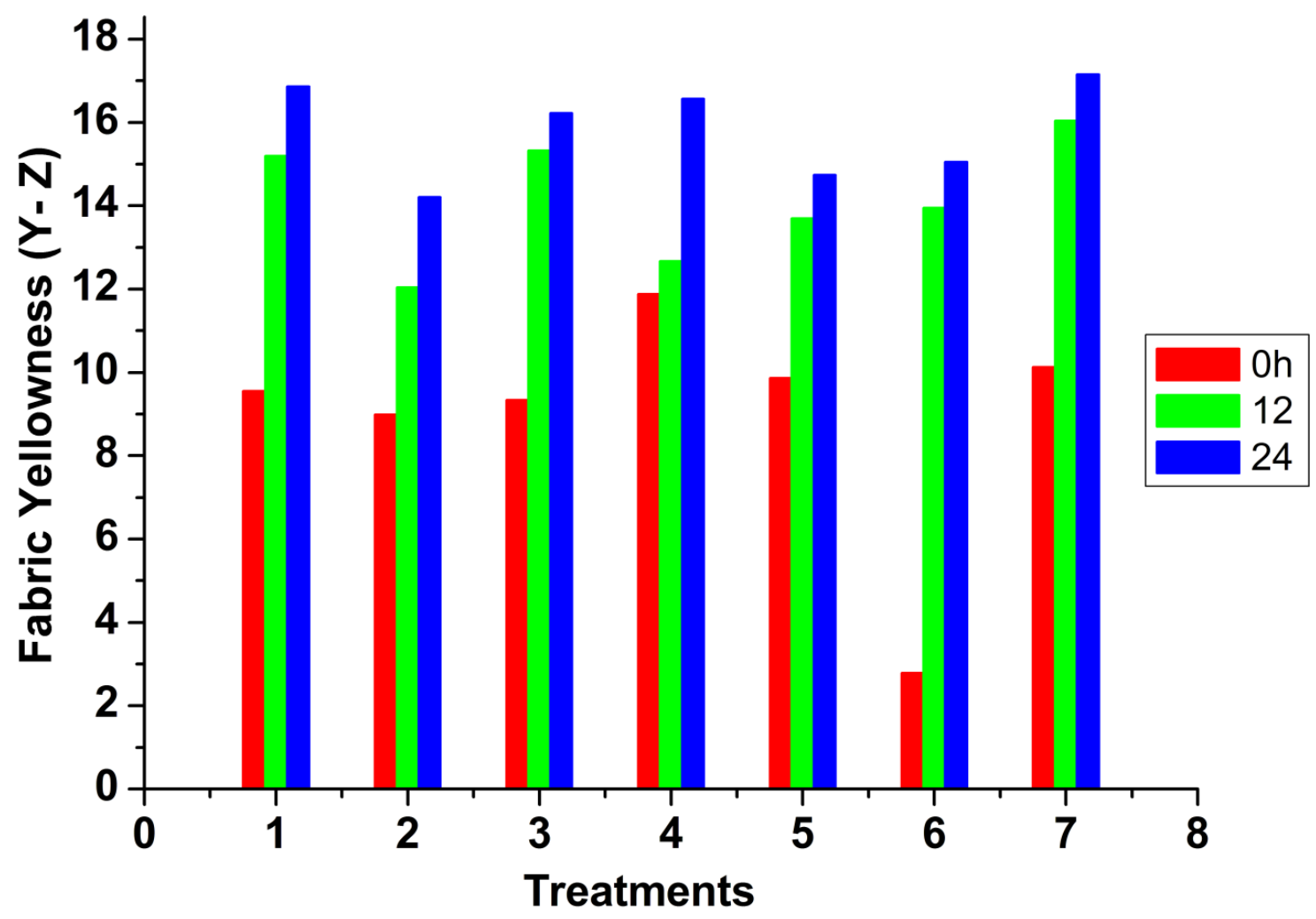

Figure 5.6: CIE Y-Z (yellowness) fabric scores before and after $12 \mathrm{~h}$ and $24 \mathrm{~h}$ UVB irradiation, with a low concentration of nanoparticles $(2.106 \times 1015$ particles $/ \mathrm{ml})$ dispersed in the matrix. (1) Control (untreated fabric); (2) matrix treated; (3) matrix + Ge-amine; (4) matrix + Ge-alkene; (5) matrix + Si-amine; (6) matrix + Si-alkene; (7) matrix + Uvitex.

The $\mathrm{Y}-\mathrm{Z}$ values of fabrics exposed to UVB radiation after coating with Si-matrix and a low concentration of nanoparticles $\left(2.106 \times 10^{15}\right.$ particles $\left./ \mathrm{ml}\right)$ is shown in Figure 5.6. At $12 \mathrm{~h}$ and $24 \mathrm{~h}$ of UVB radiation, the fabric is seen to yellow drastically, with the Uvitex treatment undergoing maximum yellowing. 


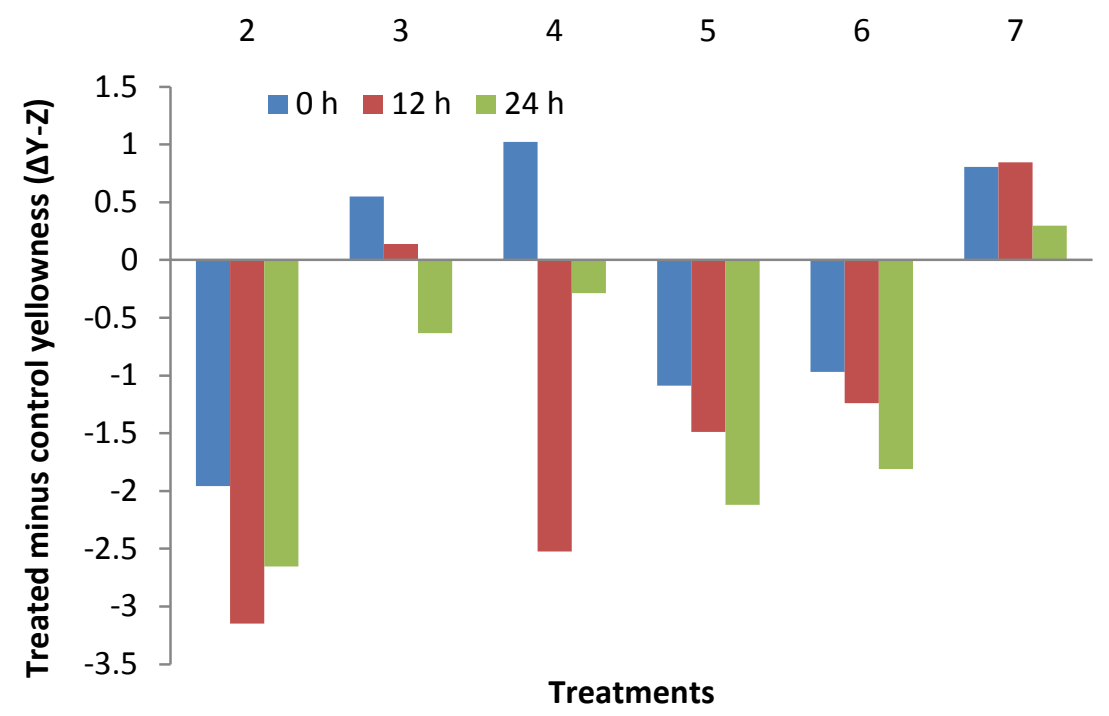

Figure 5.7: $\triangle$ CIE Y-Z fabric scores (sample Y-Z minus control Y-Z (2) Matrix treated; (3) Matrix + Ge-amine; (4) Matrix + Ge-alkene; (5) Matrix + Si-amine; (6) Matrix + Sialkene; (7) Matrix + Uvitex.

The CIE Y-Z values after matrix treatment with high concentration of nanoparticles $\left(6.318 \times 10^{15}\right.$ particles $\left./ \mathrm{ml}\right)$ and exposed to UVA radiation are displayed in Figure 5.8 and the differences in CIE Y-Z value between the treated fabric and the control are displayed in Figures 5.9. The CIE Y-Z values after UVB radiation are displayed in Figure 5.10 and the differences in CIE Y-Z value in Figure 5.11. 


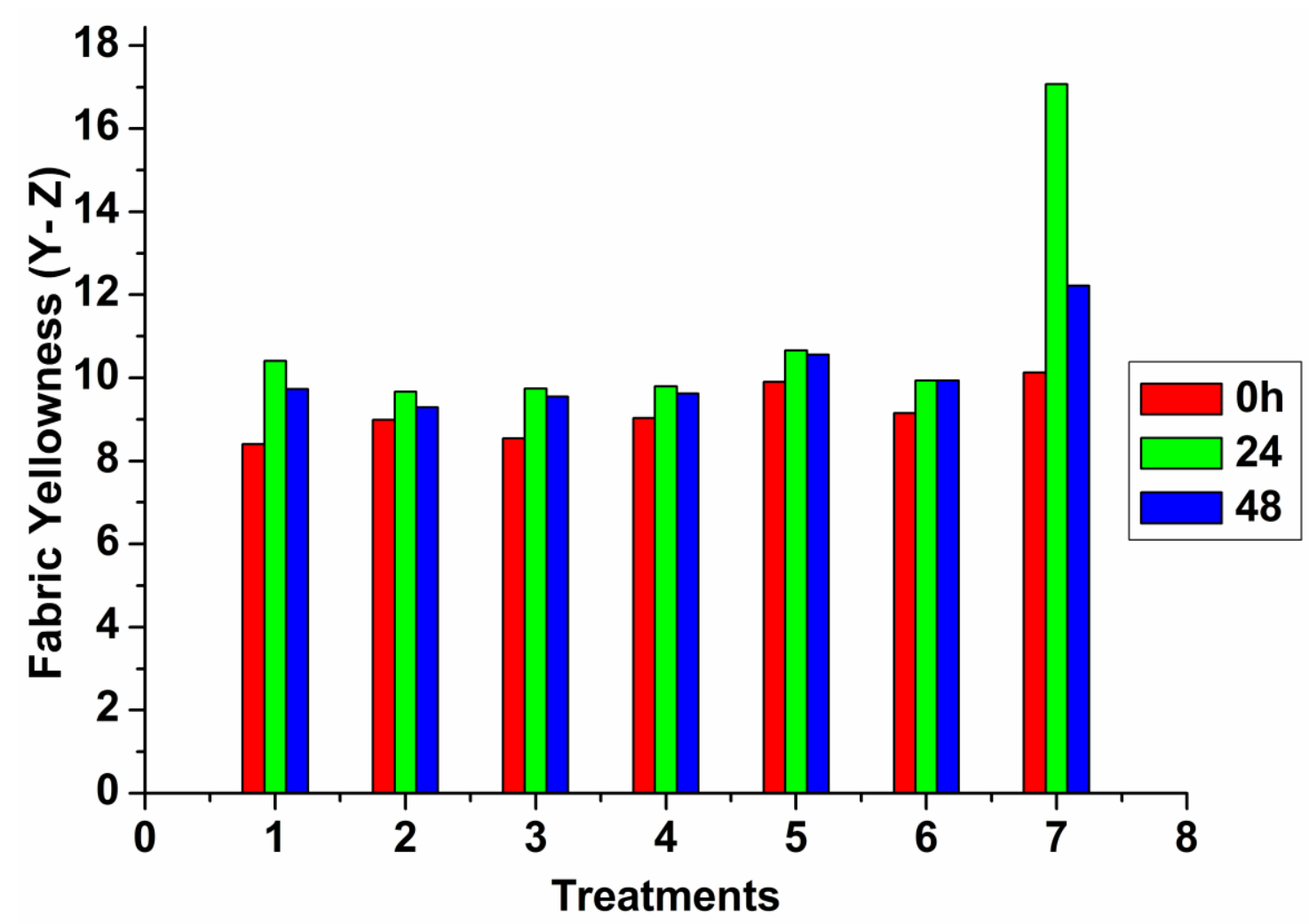

Figure 5.8: $\mathrm{Y}-\mathrm{Z}$ (yellowness) fabric scores before and after $24 \mathrm{~h}$ and $48 \mathrm{~h}$ UVA irradiation, with a high concentration of nanoparticles $(6.318 \times 1015$ particles $/ \mathrm{ml})$ dispersed in the matrix. (1) Control (untreated fabric); (2) matrix treated; (3) matrix + Ge-amine; (4) matrix + Ge-alkene; (5) matrix + Si-amine; (6) matrix + Si-alkene; (7) matrix + Uvitex.

Figure 5.8 gives the $\mathrm{Y}-\mathrm{Z}$ values of fabric exposed to UVA radiation, with a high concentration of fluorescent whitening agents. At $24 \mathrm{~h}$ of exposure to radiation, the fabric with Uvitex treatment had deteriorated drastically, with a Y-Z value of about 17. Treatments $1-5$, though relatively stable at $24 \mathrm{~h}$ exposure, showed signs of photobleaching after $48 \mathrm{~h}$. 
However treatment 6 (Si-hexene), remained stable even after $48 \mathrm{~h}$ of exposure to radiation.

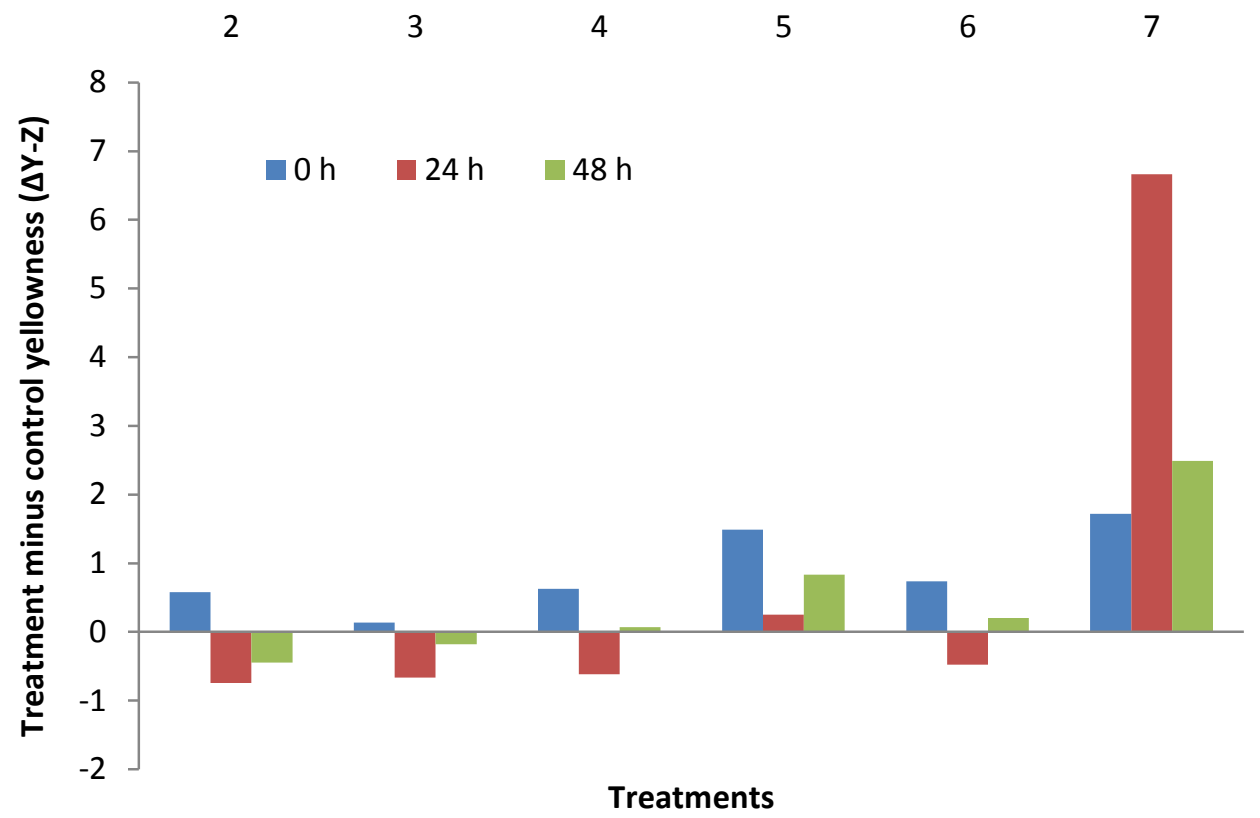

Figure 5.9: $\Delta$ CIE Y-Z fabric scores (sample $Y-Z$ minus control $Y-Z$ (2) Matrix treated; (3) Matrix + Ge-amine; (4) Matrix + Ge-alkene; (5) Matrix + Si-amine; (6) Matrix + Sialkene; (7) Matrix + Uvitex. 


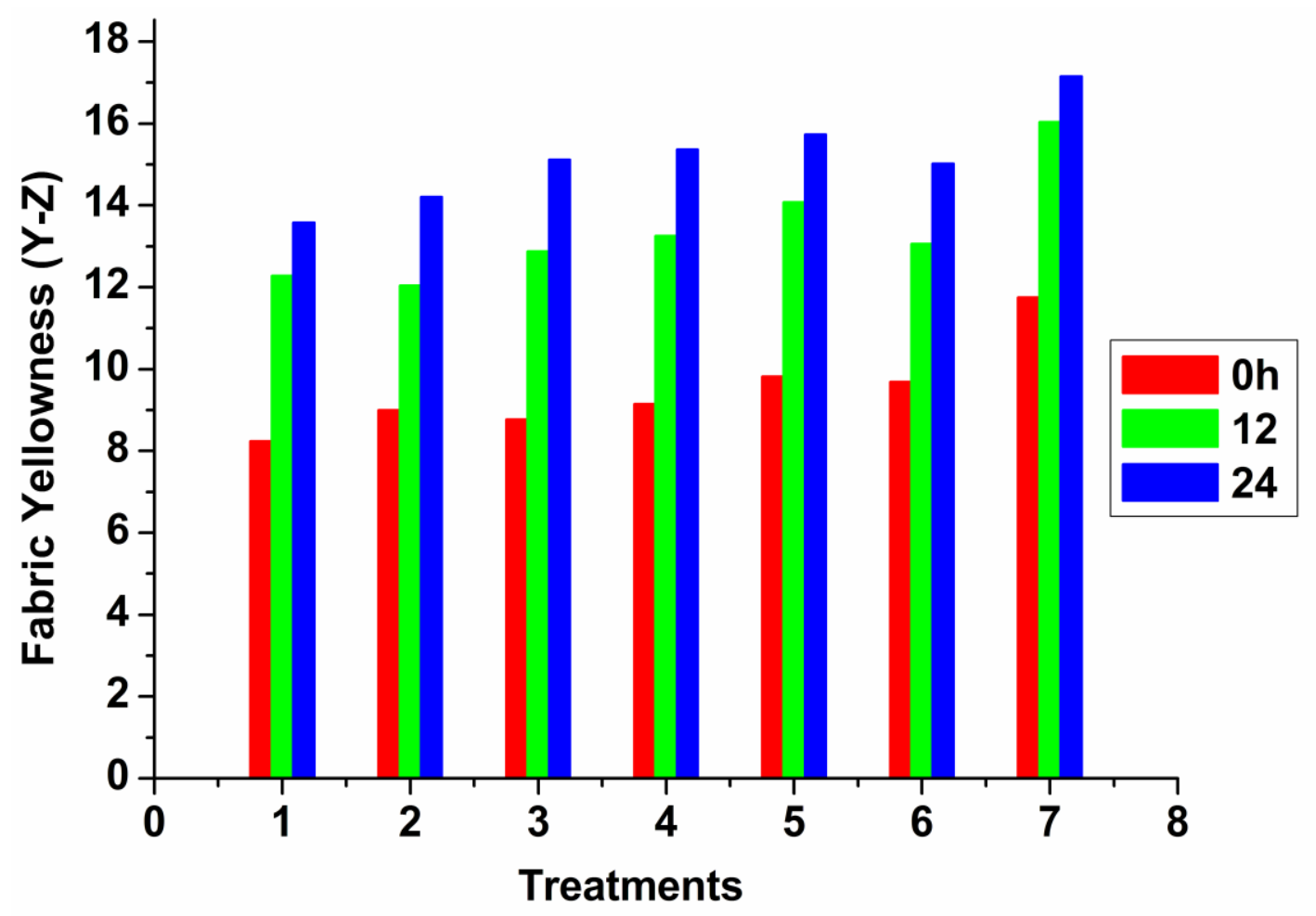

Figure 5.10: $\mathrm{Y}-\mathrm{Z}$ (yellowness) fabric scores before and after $24 \mathrm{~h}$ and $48 \mathrm{~h}$ UVB irradiation, with a high concentration of nanoparticles $(6.318 \times 1015$ particles $/ \mathrm{ml})$ dispersed in the matrix. (1) Control (untreated fabric); (2) matrix treated; (3) matrix + Ge-amine; (4) matrix + Ge-alkene; (5) matrix + Si-amine; (6) matrix + Si-alkene; (7) matrix + Uvitex.

Figure 5.10 gives the yellowness scores of fabric coated with a high concentration of fluorescent whitening agents and exposed to UVB radiation. At $0 \mathrm{~h}$ exposures, most fabric treatments had yellowness values in the region of about 8-8.5, however even before the fabric could be exposed to radiation the Uvitex coated fabric had started to yellow rapidly. At $24 \mathrm{~h}$ exposures, a similar trend was observed, the Uvitex coated fabric experiencing even more yellowing. After $48 \mathrm{~h}$ of UVB radiation, the nanocrystal coated 
fabrics showed increased yellowing; however the Uvitex coated fabric showed maximum levels of yellowing.

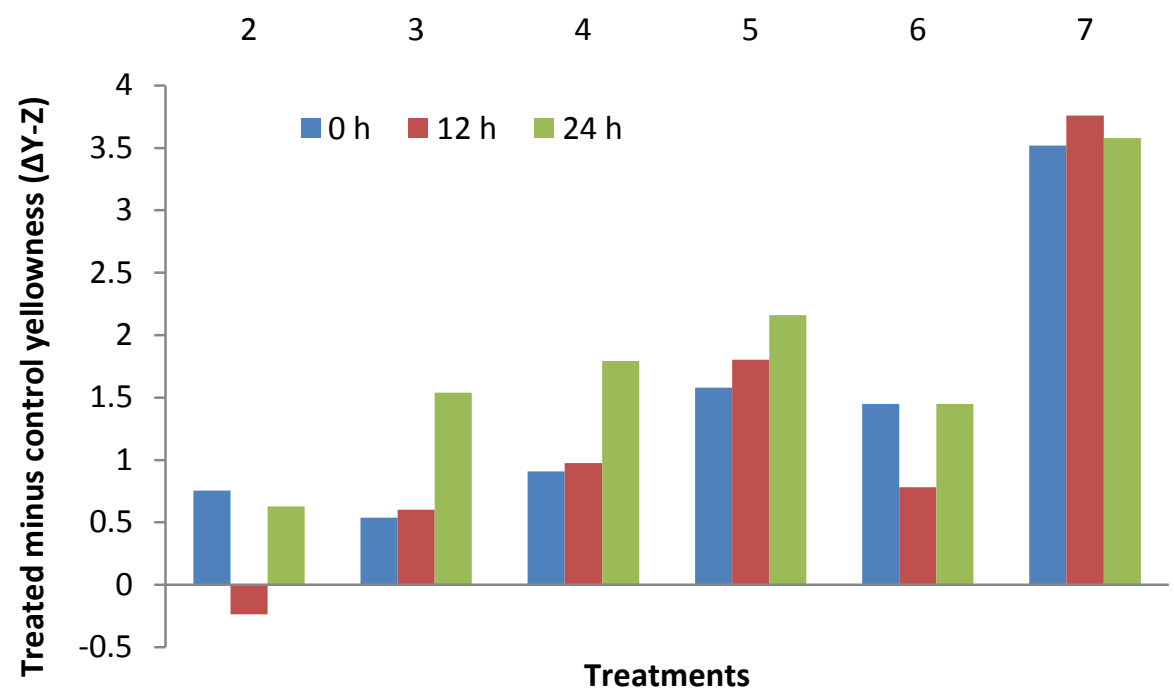

Figure 5.11: $\Delta$ CIE Y-Z fabric scores (sample Y-Z minus control Y-Z (2) Matrix treated; (3) Matrix + Ge-amine; (4) Matrix + Ge-alkene; (5) Matrix + Si-amine; (6) Matrix + Si-alkene; (7) Matrix + Uvitex.

As expected, the samples displayed varying degrees in Y-Z (yellowness) before and after exposure to UVA or UVB irradiation. Some treatments were observed to impart a beneficial pre-irradiation whitening effect. These can be seen as negative values at $0 \mathrm{~h}$ UVA or UVB exposure in figures 5.5, 5.7. 5.9 and 5.11 (or lower initial $\mathrm{Y}-\mathrm{Z}$ than the control in Figures 5.4, 5.6. 5.8 and 5.10). Pre-irradiation whitening effects were highest with Si-Hexene nanoparticles (low concentration) incorporated in the matrix (figure 5.5, treatment 6) with a value of -7 (negative values indicate that they are less yellow than control). Si-Hexene treated fabrics maintained their whiteness advantage over untreated fabric even after $48 \mathrm{~h}$ UVA exposure (this equates to three or four years worth of regular sunlight exposure through window glass) ${ }^{9}$. 
The improved photostability of some of the treatments at lower concentrations is evident in figures 5.4 through 5.7. There was noticeable improvement in photostability during UVB radiation for $\mathrm{Si}$-Amine and $\mathrm{Si}-\mathrm{Hexene}$ treatments applied at lower concentrations (Figures 5.6 and 5.7, treatments 5 and 6) with values of 14 and 15 respectively. While fabrics with these treatments became more yellow ( Figure 5.6) they did so more slowly than untreated fabric, while maintaining a whiteness advantage over Uvitex-treated fabric during 12 and $24 \mathrm{~h}$ UVB exposure, thereby demonstrating superior colour stability. Under UVA irradiation, Si-Amine treated fabric, in particular, yellowed very little over 48h exposures to UVA. Ge-Hexene (Figure 5.5, treatment 4) also demonstrated a photoprotective effect against UVA when applied at lower concentration, as can be seen by only slight increases in yellowing (negative trend) compared to the control. GeAmine also displayed photostability over $24 \mathrm{~h}$ UVB exposures as can be seen in Figures 5.6 and 5.7 treatment 3$)$.

FWA treatments applied at higher concentrations (Figure 5.8 and 5.9), imparted excellent colour stability to UVA over $48 \mathrm{~h}$ (yellowness of $\sim 10-11$ ), with the exception of Uvitex (yellowness of 17), which yellowed considerably after 24h. Against UVB irradiation, treatments applied at the higher concentration were not observed to exert a photoprotective effect (Figures 5.10 and 5.11). Generation of reactive oxygen species by high concentration of nanoparticles have been reported earlier ${ }^{10}$ and an increase in the yellowness index can be attributed to the generation reactive oxygen species which can exacerbate the photoyellowing process. This matches with the results obtained from the luminol assay, described later in section 5.5, where a clear evidence of luminol degradation is seen in the presence of both Ge and Si nanoparticles. 
Significantly lower number of nanoparticle treatments at the lower concentration were observed to display worse initial whiteness and increased photoyellowing in comparison to the control (Figures 5.5 and 5.7). Uvitex treated fabrics were observed to display much yellowed colouration than fabrics treated with nanoparticle fluorescent whitening agents (Figures 5.5, 5.7, 5.9 and 5.9). Overall, Si-Amine and Si-Hexene treatments at the lower concentration (treatments 5 and 6, yellowness values range of 2-14) demonstrated good photostability, whitening and improved performance in comparison to untreated fabric and Uvitex treated fabric.

Uvitex, compared to the nanoparticles, showed the highest increase in yellowness index. Overall against UVA irradiation, the Si-amine and Si-alkene and against UVB Gealkene, Si-amine and Si-alkene, at the low concentration, were similar in their photoprotective effect compared to the matrix treatment alone. Both of these attributes, whitening and photoprotection, are desirable features of high value textiles, and hold significant potential for further development. 


\subsection{HPLC Assay Evaluating the Oxidative Degradation of Luminol}

Reactive oxygen species (ROS) are known to be generated by organic FWAs, thereby exacerbating the photoyellowing process in the wool. At high concentration, nanoparticles are also reported to generate $\operatorname{ROS}^{11}$. The generation of singlet oxygen is known to photosensitize wool through the formation of highly reactive intermediates ${ }^{11,12}$. Here, an assay was adapted to evaluate the levels of singlet oxygen generated by the inorganic FWA's, with correlation to the levels generated by the reference stilbene-based organic FWA, Uvitex NFW.

This HPLC assay is based on monitoring singlet oxygen generation via the degradation of luminol (LUM) to aminophthalic acid (APA) ${ }^{14,15}$. The formation of APA from LUM is shown in schematic form in Figure 5.12. Oxidative decomposition of luminol (LUM) was monitored in the presence of the inorganic FWAs, rose bengal (a well-known photosensitiser ${ }^{14}$ ), and Uvitex NFW (a commercially available organic FWA), before and after exposure to 1,6 and $12 \mathrm{~h}$ of UV irradiation. 
<smiles>Nc1cccc2c(=O)[nH][nH]c(=O)c12</smiles>

Luminol

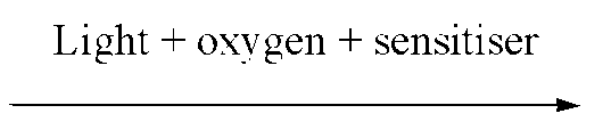

Aminophthalic acid

Figure 5.12: Structures of luminol and its degradation product, aminophthalic acid [11].

Analysis of HPLC chromatogram traces demonstrated the formation of APA as a result of photo-oxidation of LUM, with almost total degradation of LUM after $6 \mathrm{~h}$ of UVA irradiation (Figure 5.13).

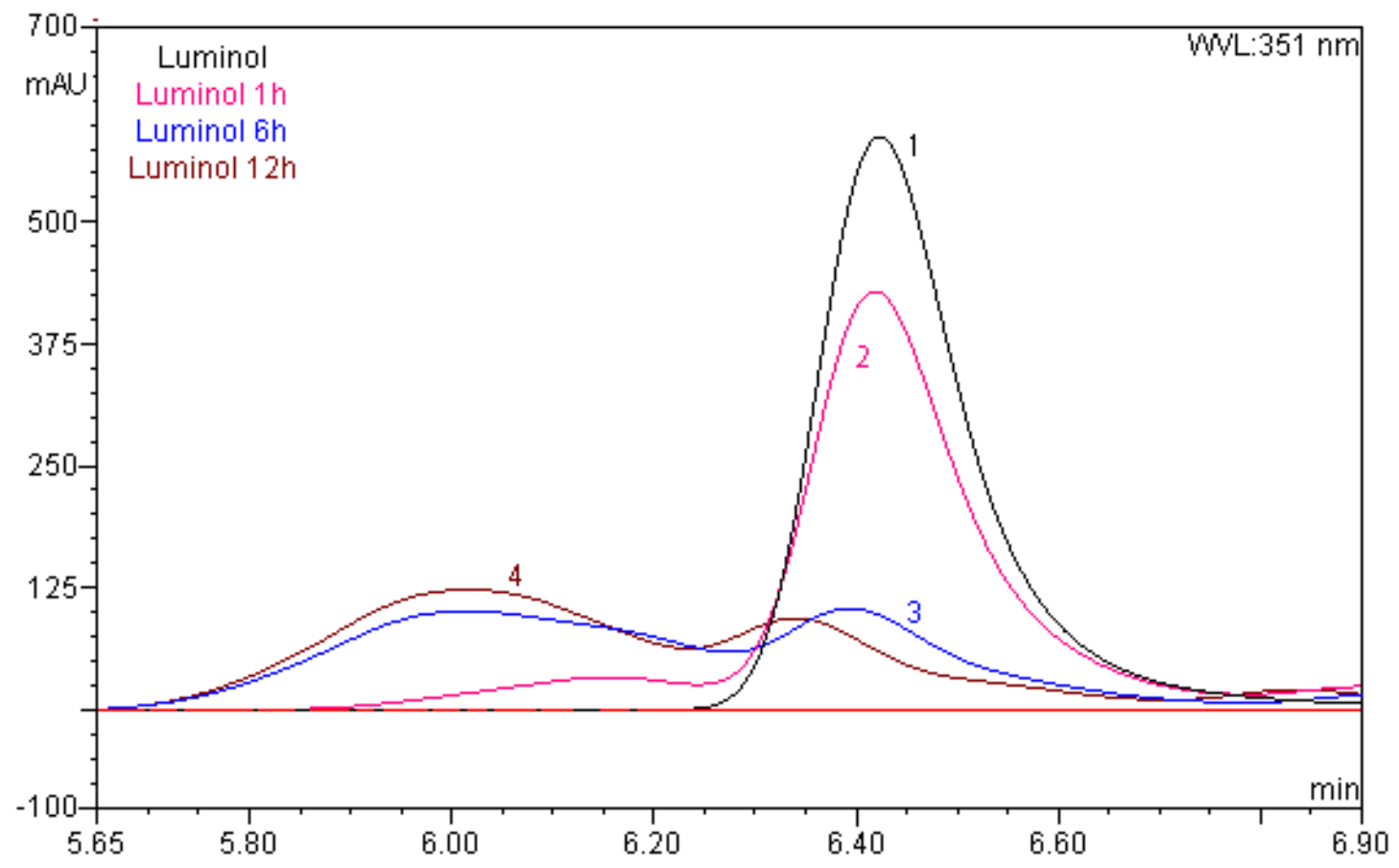

Figure 5.13: HPLC spectrum demonstrating the photodecomposition of LUM to APA after $0 \mathrm{~h}$ (black trace 1), $1 \mathrm{~h}$ (pink trace 2), $6 \mathrm{~h}$ (blue trace 3 ) and $12 \mathrm{~h}$ (brown trace 4 ) irradiation with UVA. 


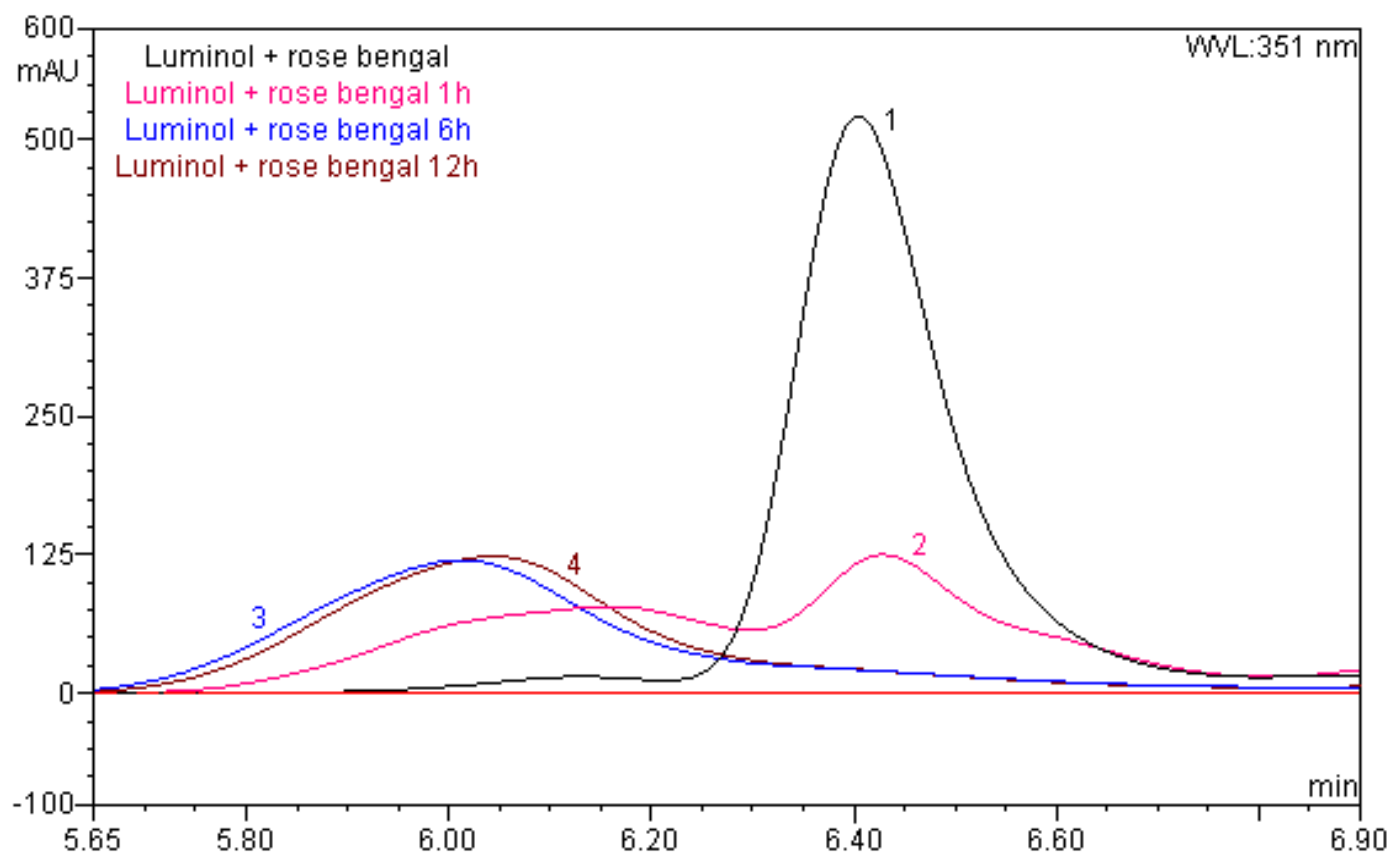

Figure 5.14: HPLC spectrum showing the formation of APA from LUM in the presence of rose bengal irradiated with UVA for $0 \mathrm{~h}$ (black trace 1), $1 \mathrm{~h}$ (pink trace 2), $6 \mathrm{~h}$ (blue trace 3 ) and $12 \mathrm{~h}$ (brown trace 3 ) in the presence of rose bengal.

The singlet oxygen-generator, rose bengal, as expected, was seen to exacerbate LUM degradation upon UVA exposure, as demonstrated by its considerable degradation after only $1 \mathrm{~h}$ irradiation (pink trace 2). When irradiated for $12 \mathrm{~h}$ in the presence of the singlet oxygen-generator, rose bengal, LUM degradation was almost complete, with corresponding APA formation. 
The addition of an antioxidant, L-ascorbic acid, used as an inhibitor of ROS generation, reduced LUM degradation to a considerable extent (Figure5.15, black trace 1). These results effectively validated the assay.

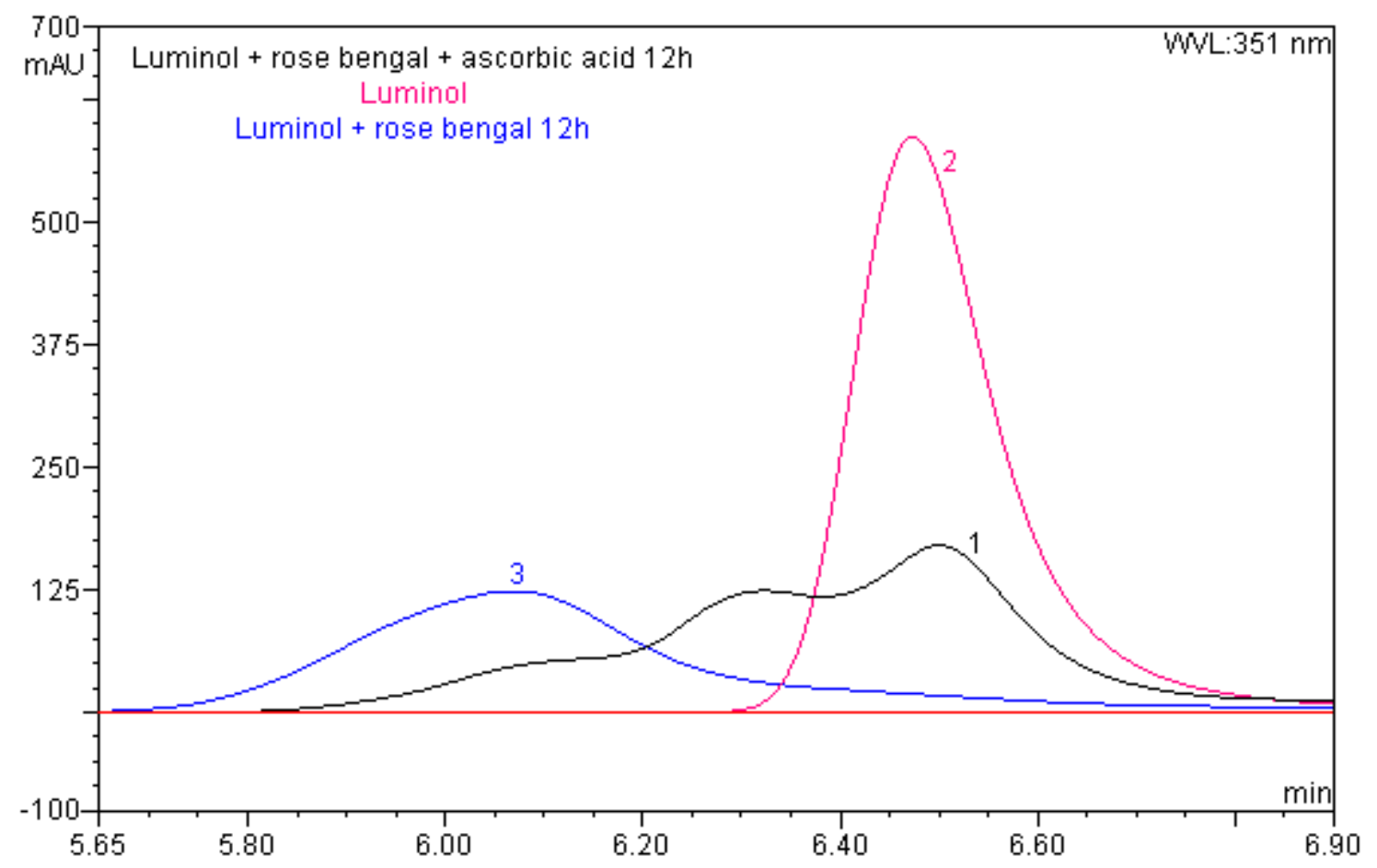

Figure 5.15: HPLC spectrum demonstrating the formation of APA from LUM (pink trace 1) after $12 \mathrm{~h}$ UVA irradiation in the presence of the sensitizer, rose bengal (blue trace 3), and the inhibition of complete photodecomposition in the presence of ascorbic acid (black trace 1).

In the presence of the organic FWA, Uvitex NFW, a significant degradation of LUM to APA is evident after $6 \mathrm{~h}$ and $12 \mathrm{~h}$ UVA irradiation (Figure 5.16, blue trace 3). A smaller reduction in the LUM peak is seen after $1 \mathrm{~h}$. ROS generation in response to Uvitex application is driven by the formation of Uvitex degradation products, which this assay 
indicates may have taken more than one hour to form in substantive quantities. The longer-term results seen after 6 and 12 hours are more conclusive.

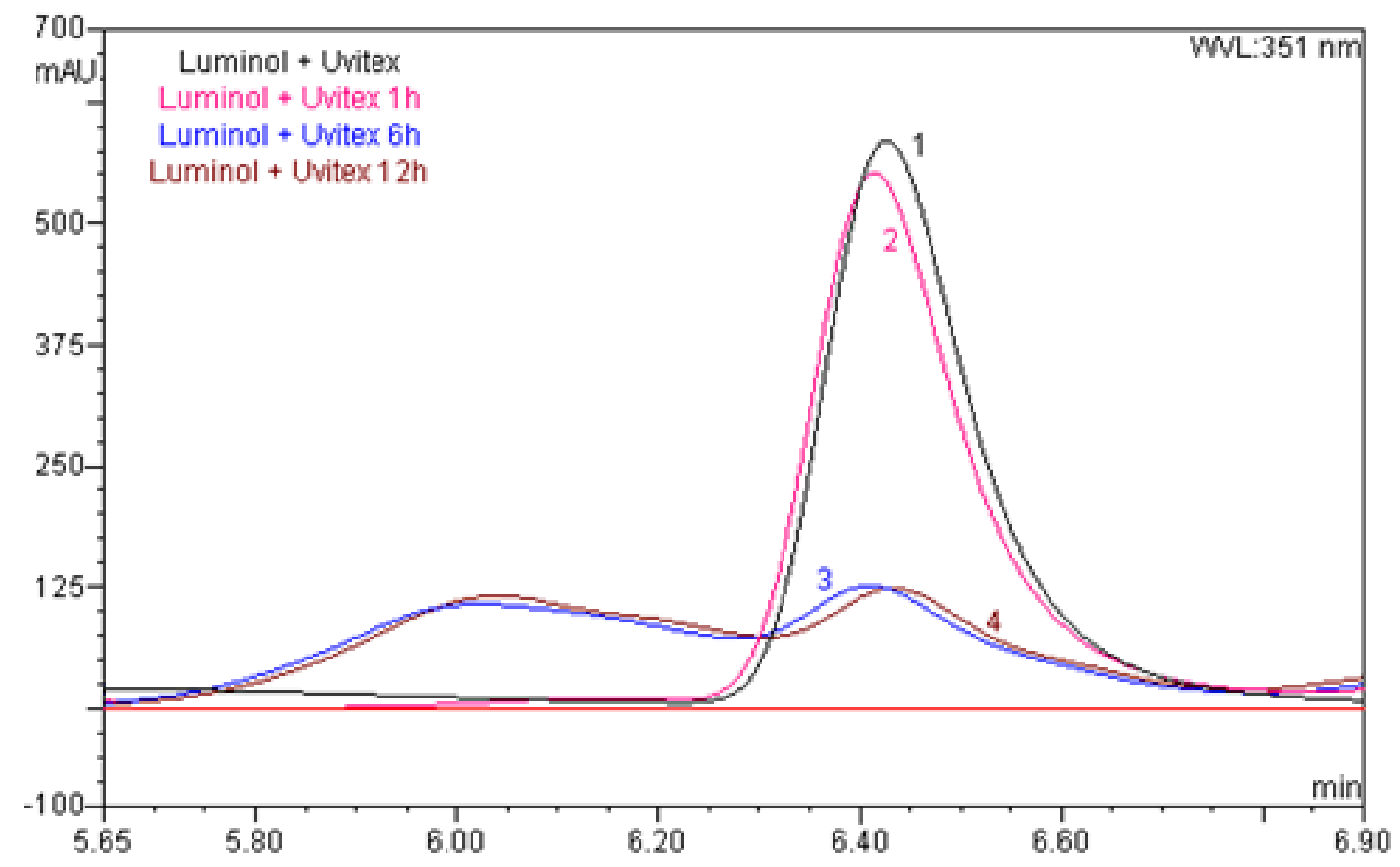

Figure 5.16: HPLC spectrum demonstrating the increased stability of LUM to singlet oxygen induced degradation in the presence of the organic FWA, Uvitex, after irradiation with UVA for $0 \mathrm{~h}$ (black trace 1), $1 \mathrm{~h}$ (pink trace 2), $6 \mathrm{~h}$ (blue trace 3) and $12 \mathrm{~h}$ (brown trace 4).

LUM degradation by singlet oxygen during UVA irradiation was also observed in the presence of high concentrations $(6.318 \times 1015$ particles $/ \mathrm{ml})$ of the inorganic Si-amine and Ge-amine nanoparticles (Figures 5.17 and 5.18). After $12 \mathrm{~h}$ UVA exposure, the percentage of LUM degradation by Uvitex was higher than both Si-amine and Ge-amine nanoparticles $(6.318 \times 1015$ particles $/ \mathrm{ml})$. Approximately $59 \%$ of the LUM degraded to 
APA in the presence of Uvitex, higher than the $45 \%$ and $26 \%$ degradation observed in the presence of Si-amine and Ge-amine nanoparticles, respectively. The Ge nanoparticles showed the least generation of singlet oxygen.

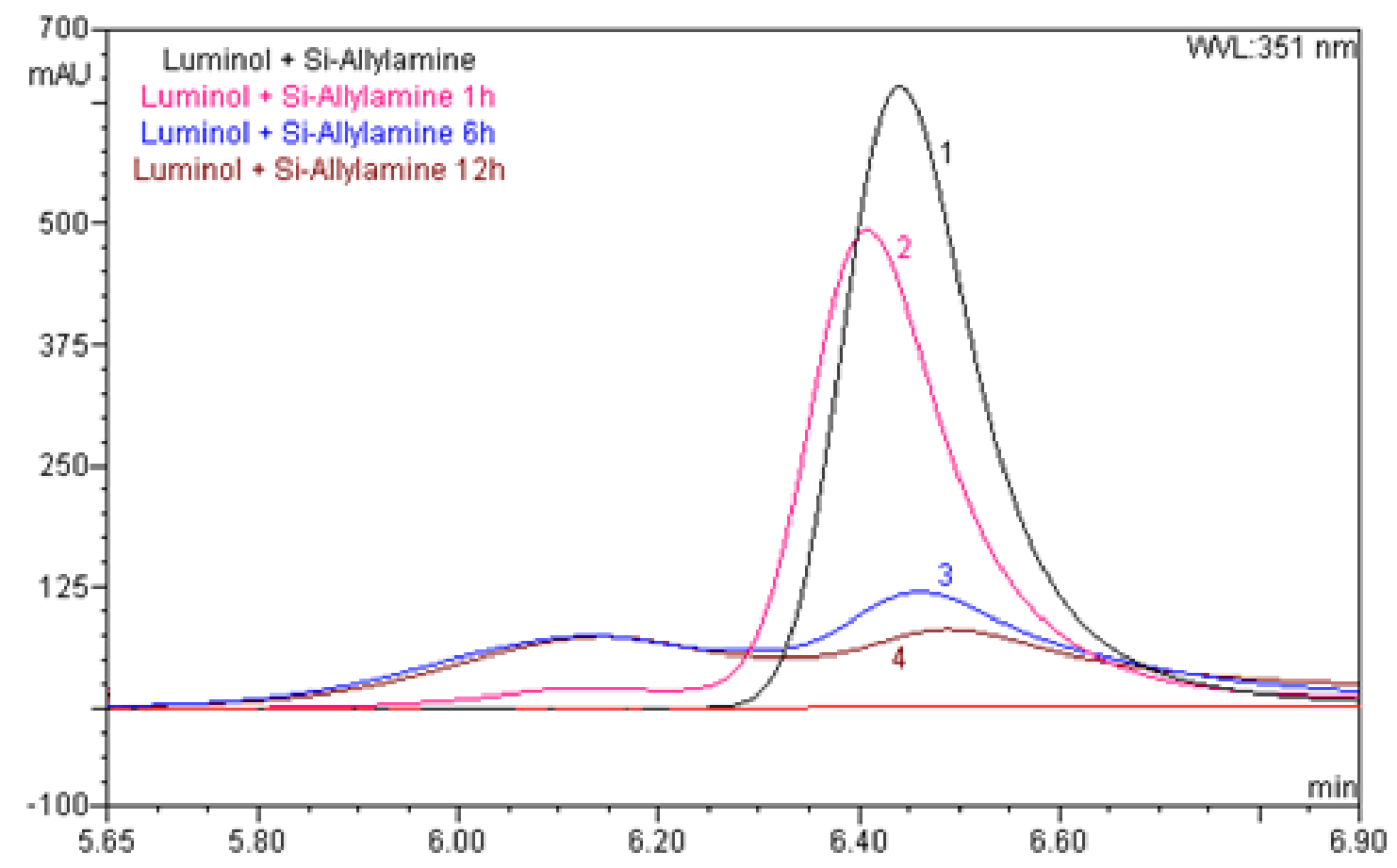

Figure 5.17: HPLC spectrum demonstrating the reduction of LUM peak area after irradiation with UVA for $0 \mathrm{~h}$ (black trace 1), $1 \mathrm{~h}$ (pink trace 2), $6 \mathrm{~h}$ (blue trace 3) and 12 h (brown trace 4) in the presence of a high concentration of Si-amine nanoparticles $(\sim 6.318 \times 1015$ particles $/ \mathrm{ml})$. 


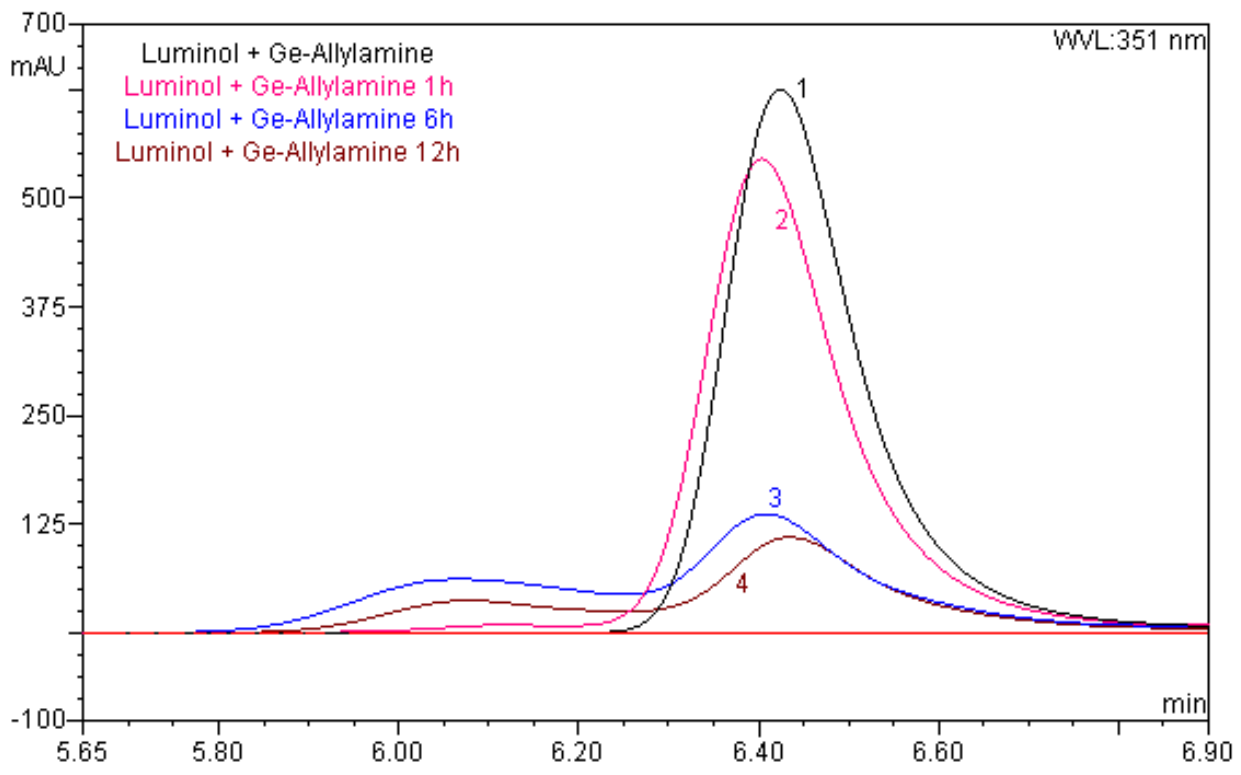

Figure 5.18: HPLC spectrum demonstrating the reduction of LUM peak area after irradiation with UVA for $0 \mathrm{~h}$ (black trace 1), $1 \mathrm{~h}$ (pink trace 2), $6 \mathrm{~h}$ (blue trace 3) and 12 $\mathrm{h}$ (brown trace 4) in the presence of a high concentration of Ge-amine nanoparticles $(\sim 6.318 \times 1015$ particles $/ \mathrm{ml})$. 


\subsection{Electron Microscopy Studies on Wool 5.6.1 Scanning Electron Microscopy analysis}

Scanning electron microscope (SEM) images were taken of fabric samples before and after coating with the matrix and with the higher nanoparticle concentration. Fibres from untreated fabric exposed to $120^{\circ} \mathrm{C}$ for $1 \mathrm{~h}$ showed scale damage and exposed cortical structure (Figures 5.19a and b). The matrix coating reduced such damage and gave good results with the matrix coverage more pronounced on top of the weave (Figures $5.19 \mathrm{~b}$ ).

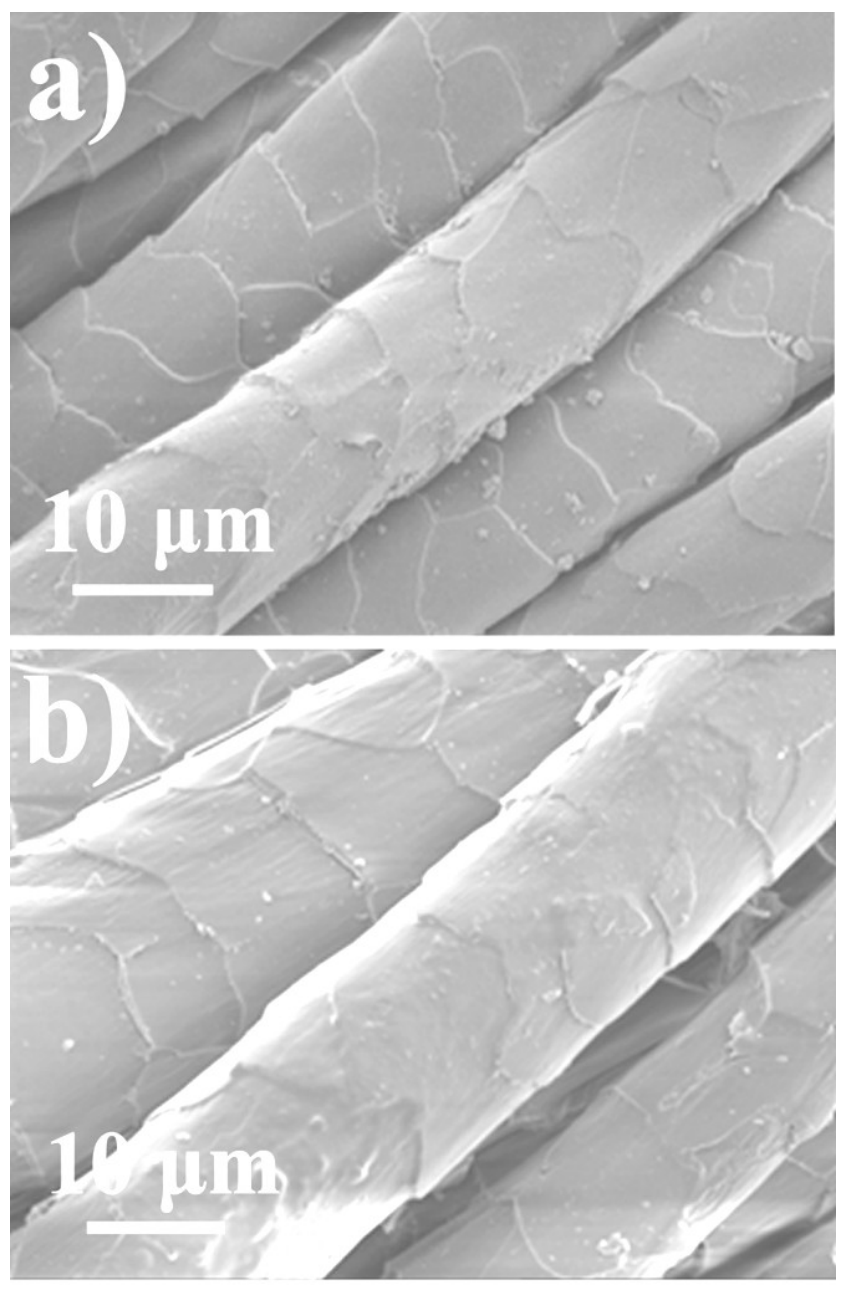

Figure 5.19: SEM image of (a) untreated fabric (b) sol-gel coated fabric 
The treated fibers do not show any obvious cracks in the matrix when observed at the higher magnifications, with the matrix coating both the top and sides of the fabric, though lesser on the sides. When nanoparticle or Uvitex FWAs were added to the matrix, the patchiness of the coating was observed to be greater (Figure 5.20 a to e). Minimal ('sticky') coating was observed along and between treated fibers. The coating did not completely obscure scale definition with no observable detachment of the matrix from the fiber surface indicating a thin (desirable) distribution of the coating on the fiber surface. 

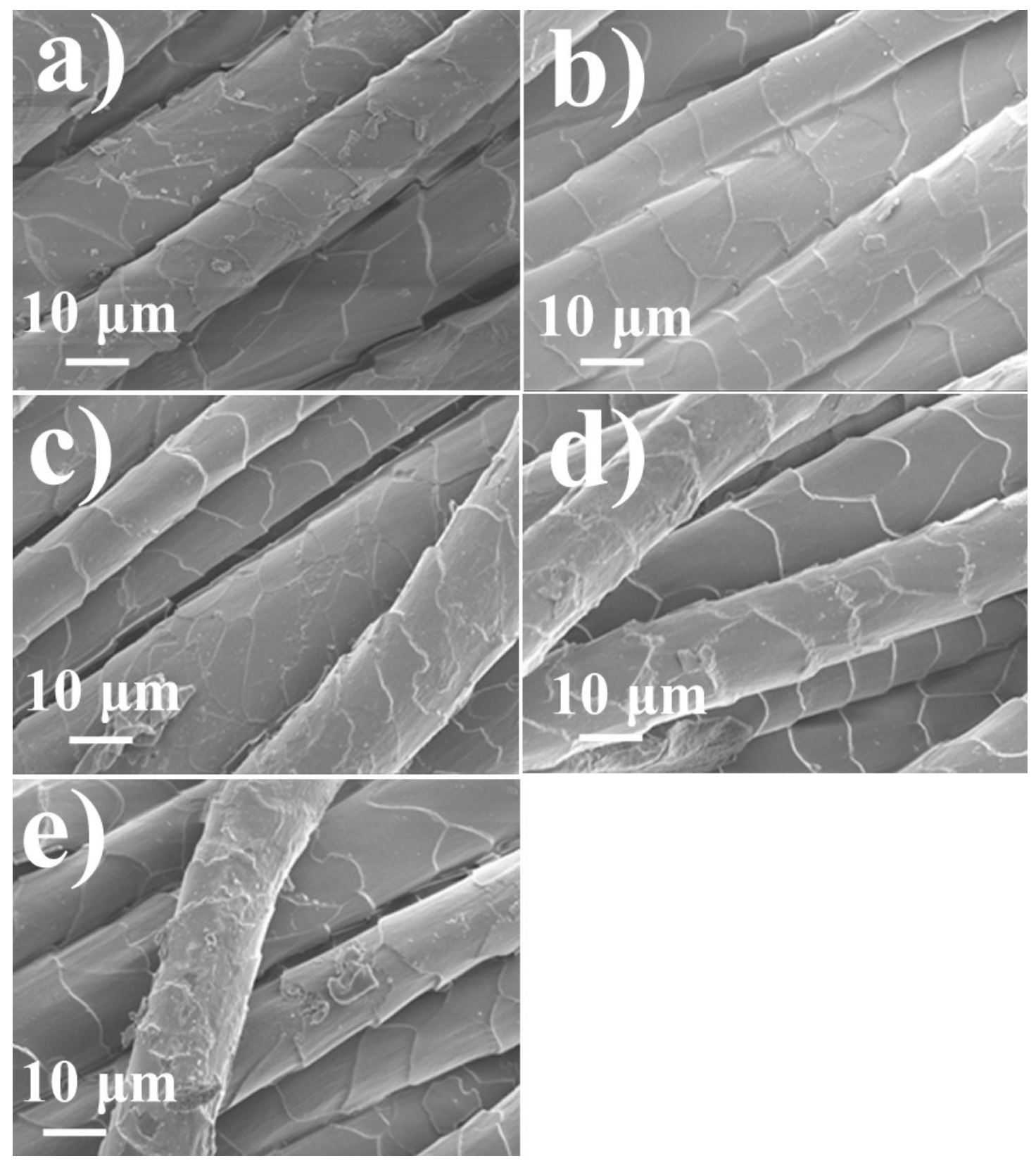

Figure 5.20: SEM image of wool fabric coated with (a) Ge-amine, (b) Ge-Hexene, (c)

Si-amine, (d) Si-Hexene and (e) Uvitex 


\subsubsection{STEM/TEM Studies of Ge-amine Treated Wool Fiber}

Scanning transmission electron microscopy (STEM), implemented on a conventional transmission electron microscope with STEM-attachment, has been used as a primary tool to investigate the nature and composition of the coated fabric using EDX mapping.

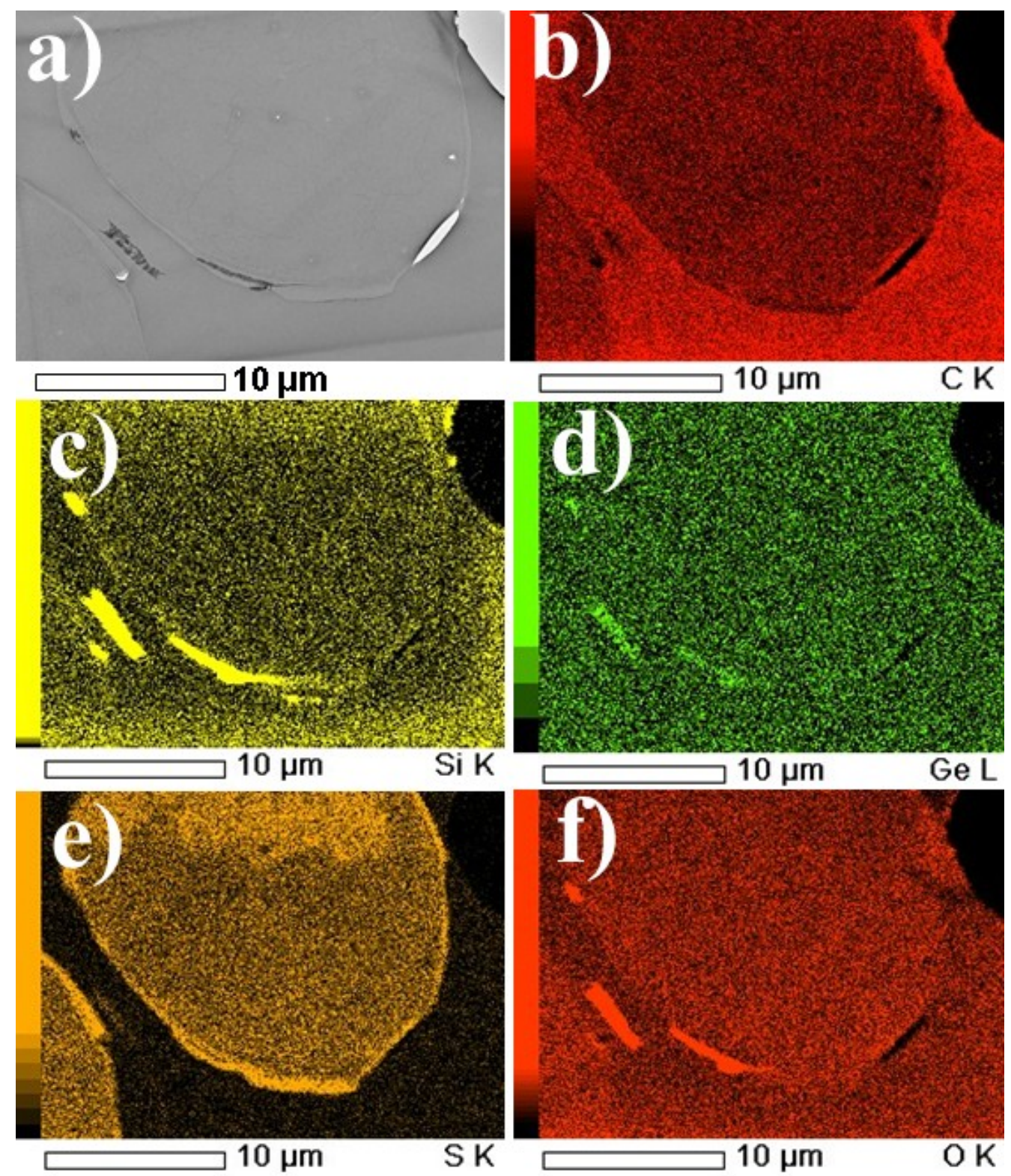

Figure 5.21: (a) TEM image of a section of germanium 1-hexene nanoparticle coated fabric and (b-f) the elements that constitute the fabric. 
Due to the sol-gel matrix containing silicon by itself, an EDX map of silicon nanoparticle coated fibers would prove futile. Therefore germanium nanoparticles coated fabrics were used in this study. Figure 5.21a is a TEM image of a section of germanium 1hexene coated wool fabric. Due to the presence of a silicon based sol-gel matrix, high resolution TEM of the fiber was not possible. Figures 5.21c, e and $\mathrm{f}(\mathrm{C}, \mathrm{O}$ and $\mathrm{S})$ are EDX maps of the wool fiber in figure 5.21a and are the basic elements that constitute wool. The $\mathrm{Si}$ in figure 5.21c is from the sol-gel matrix, whilst the germanium is from the nanoparticle coating in the fiber, thus verifying that a uniform coating of germanium nanoparticles is possible using this method. 


\subsection{Summary}

Surface-modified nanoparticles with good photostability against UVA and UVB relative to Uvitex, as well as excellent blue light fluorescence characteristics have been synthesized. Both the Si and Ge nanoparticles have diameters smaller than the bulk excitation Bohr radius, resulting in enhanced photoluminescence in the visible range. The results from this study are summarized as follows.

Both the Si and Ge nanoparticles displayed matching intense blue fluorescence when excited at $400 \mathrm{~nm}$, both in dry and aqueous states and when applied to the fabric, the emission range of the nanoparticles was more centralized in the visible range than that of the organic FWA, Uvitex; this effect will generate a brighter fabric. The emission properties of the nanoparticles are therefore demonstrated to be equally or more suited to improving the brightness properties of fabric than Uvitex. This result confirms that these inorganic nanoparticles possess desirable emission properties for use as commercial FWAs.

Under UVA irradiation, LUM was observed to be degraded in the presence of both Uvitex and amine-capped nanoparticles at high concentration. Over $12 \mathrm{~h}$ UVA, 59\% of the LUM degraded to APA in the presence of Uvitex, while over the same time period, only $45 \%$ and $26 \%$ of LUM $\rightarrow$ APA degradation was observed for Si-amine and Geamine nanoparticles, respectively. This reflects reduced generation of singlet oxygen by the nanoparticles, compared to the organic FWA, Uvitex, particularly in the case of the Ge-amine nanoparticles. Reduced ROS generation is a desirable attribute for new generation whitening and brightening agents. 


\subsection{References}

1. Higginbotham, R. S.; Thomas, F. W., Fluorescent whitening agents, Nature 1958, 181 1437-1439.

2. Dyer, J.M., Cornellison, C.D., Bringans, S.D., Maurdev, G., Millington, K.R., The photoyellowing of stilbene-derived fluorescent whitening agentsmass spectrometric characterization of yellow photoproducts. Photochem. Photobiol. 2008; $84: 145-53$

3. Davidson, R.S., The photodegradation of some naturally occurring polymers, $J$. Photochem. Photobiol. B 1996 33, 3-25.

4. Dyer, J.M.; Bringans, S. D.; Bryson, S.D., Determination of photo-oxidation products within photoyellowed bleached wool proteins J. Photochem. Photobiol. B $200682,551-557$.

5. Dyer, J.M.; Bringans, S. D.; Bryson, S.D., Characterisation of photo-oxidation products within photoyellowed wool proteins: tryptophan and tyrosine derived chromophores, Photochem. Photobiol. Sci. 2006 5, 698-706.

6. Dyer, J. M.; Cornellison, C. D.; Bringans, S. D.; Maurdev, G.; Millington, K. R., The photoyellowing of stilbene-derived fluorescent whitening agents- 
mass spectrometric characterization of yellow photoproducts, Photochem. Photobiol. 200884 145-153

7. Warner J. H.; Dunlop H-R.; Tilley R. D., Surface morphology dependent photoluminescence from colloidal silicon nanocrystals, $J$ Phys Chem $B$ 2005, 109(41), 19064-19067.

8. Warner, J. H.; Hoshino, A.; Yamamoto, K.; Tilley, R. D., Water-soluble photoluminescent silicon quantum dots, Angew. Chem. Int. Ed. 2005, 44, 45504554.

9. Tilley, R. D. W., J. H.; Yamamoto, K.; Matsui, I.; Fujimori, H., Microemulsion synthesis of monodisperse surface stabilized silicon nanocrystals, Chem. Comm. 2005, 1833-1835.

10. Chadysiene, R. S.; Girgzdiene, R.; Girzdys, A., Ultraviolet radiation and ground-level ozone variation in Lithuania, J. Env. Eng. Landscape Manag 2005 13 31-36.

11. Fujioka, K., Hiruoka, M., Sato, K., Manabe, M., Miyasaka, R., Hanada, S., Hoshino, A., Tilley, R., Manome, Y., Hirakuri, K., Yamamoto, K., Luminescent passive-oxidised silicon quantum dots as biological staining labels and their cytotoxicity effects at high concentration, Nanotechnology 2008;19:1-7. 
12. Davidson, R.S., Ismail, G.M., Lewis, D.M., Retardation of the photoyellowing of untreated wool and wool treated with fluorescent whitening agens by the action of reducing agents, J. Soc. Dyers \& Colourists 1987;103:308-13.

13. Auer, P.D., Pailthorpe, M.TB., Effect of topical applications of antioxidants and quenchers on the photoyellowing of fluorescently whitened wool fabric. Text. Res. J. 1995; 65:287-93.

14. Wierrani, F., Kubin, A., Loew, H.G., Henry, M., Spängler, B., Bodner, K., Grünberger, W., Ebermann, R., Alth, G., Photodynamic action of some sensitizers by photooxidation of luminol, Naturwissenschaften 2002;89:466-9.

15. Millington, K.R., Maurdev, G., The generation of superoxide and hydrogen peroxide by exposure of fluorescent whitening agents to UVA radiation and its relevance to the rapid photoyellowing of whitened wool. J. Photochem. Photobiol. A 2004; 165:177-85. 


\section{Solution Synthesis of Titanium Disulphide Nanostructures}

\subsection{Introduction}

This chapter deals with the synthesis of titanium disulphide nanostructures and inorganic fullerene-like nanoparticles (IF) using solution routes. The materials were prepared by high temperature solution synthesis using high boiling point solventsurfactant systems. Titanium tetrachloride or titanium isopropoxide was used as the titanium source and was reacted with elemental sulphur in the presence of organic solvents. $^{16}$

Elemental sulphur has been used in the preparation of bulk titanium disulphide such as by the direct reaction of the elements at temperatures higher than $1000^{\circ} \mathrm{C}$. $^{1}$ Nanotubes of titanium disulphide have also been prepared using elemental sulphur via chemical transport reactions at temperatures of $\sim 750^{\circ} \mathrm{C} .^{2}$ However the synthesis of titanium disulphide nanostructures in solution using elemental sulphur at temperatures lower than $300^{\circ} \mathrm{C}$ at ambient pressures, have not been previously reported.

Titanium disulphide nanotubes were first prepared in solution using a two-step process by Chen and co-workers. ${ }^{3}$

${ }^{16}$ Portions of this chapter have been previously published as Sujay Prabakar, Chris W Bumby and Richard D Tilley; Chemistry of Materials, 2009, 21 (8),pp 1725-1730. 
Titanium tetrachloride in tetrahydrofuran (THF) was used as the titanium source and sodium sulphide nonahydride $\left(\mathrm{Na}_{2} \mathrm{~S} .9 \mathrm{H}_{2} \mathrm{O}\right)$ as the sulphur source. The constituents were first reacted at $40^{\circ} \mathrm{C}$ using THF to produce nano-sized amorphous products; the products were then annealed at $200^{\circ} \mathrm{C}$ for $6 \mathrm{~h}$ to form multi-walled tubular nanostructures of $\mathrm{TiS}_{2}$.

Sulphides including hydrogen sulphide are commonly used in the solution synthesis of titanium disulphide nanostructures. The toxicity of sulphides and the difficulty of scaling up the synthesis whilst using a gaseous precursor are some of the problems that current synthetic techniques face.

The motivation of the research was to establish a non-sulphide route to produce crystalline titanium disulphide nanostructures via simple bench-top solution chemistry. Sulphides including hydrogen sulphide are commonly used in the solution synthesis of titanium disulphide nanostructures.

The toxicity of sulphides and the difficulty of scaling up the synthesis whilst using a gaseous precursor are some of the problems that current synthetic techniques face. The motivation of the research was to establish a non-sulphide route to produce crystalline titanium disulphide nanostructures via simple bench-top solution chemistry.

All experiments reported in this chapter were conducted using 1:6 titanium to sulphur molar ratio. A brief discussion on using this ratio is given in the discussion section. The aim of this research was to develop a new synthetic method and the research has been 
carried out in an explanatory manner. Nine experiments crucial in the development of the new method were selected and the corresponding results described in sections 6.1 to 6.5. Titanium isopropoxide was used as the titanium source in experiments 1 and 2 and titanium tetrachloride was used in experiments 3-9. Experiments 1 and 2 differed in the coordinating abilities of the solvent used whilst experiments 4 and 5 differed only in reaction sequences detailed in table 6.1. Experiment 3 was a unique experiment which was conducted under conditions different from that of the other experiments. It was from the results in experiment three that the idea of preparing titanium disulphide from titanium tetrachloride, elemental sulphur and the non-coordinating solvent 1-Octadecene took shape. Experiments 6 to 9 were also reactions that differed only in reaction sequences and are detailed in table 6.1. 


\subsection{The Reaction of Titanium Isopropoxide and Elemental Sulfur: The formation of Titanium Dioxide (Expt. 1 and 2)}

Experiments were carried out with the aim to synthesize crystalline nanostructures of titanium disulphide from elemental sulphur and titanium isopropoxide. However, both experiments failed to produce titanium disulphide. Partly crystalline particles of titanium dioxide (Titania) of size ranging from 100-300 nm were observed along with various other products of carbonaceous matter and were the only product that could be characterized with certainty using energy dispersive X-ray analysis. In both experiments 1 and 2 crystalline titanium dioxide particles were observed to be the predominant product.

In experiment 1, a non-coordinating solvent, 1-Octadecene was used as a solvent. 1Octadecene is a straight chain heavy hydrocarbon with 1 double bond in its structure; the absence of a coordinating functional group in its structure prevents it from acting as a surfactant, but facilitates high reaction temperatures because of its boiling point and easy removal from the as-synthesized materials whilst purifying. Titanium isopropoxide is injected into a stirring hot sulphur solution at $100^{\circ} \mathrm{C}$ and the temperature of the solution increased to $250^{\circ} \mathrm{C}$. The mixture was then reacted at that temperature for $3 \mathrm{~h}$. The product obtained from the experiment consisted of clusters of semi-crystalline $\mathrm{TiO}_{2}$ particles (figure 6.1a). High resolution electron microscopy was not possible due to the presence of impurities of carbonaceous nature. EDX analysis (figure 6.1b) showed that the materials were composed of the oxide of titanium. 
In experiment 2, a coordinating solvent oleylamine was used as solvent. Reaction conditions similar to that of experiment 1 were employed, wherein titanium isopropoxide was injected into a stirring hot sulphur solution at $100^{\circ} \mathrm{C}$ and reacted at $250^{\circ} \mathrm{C}$ for $3 \mathrm{~h}$. The products were similar to that obtained for experiment 1 and were composed predominantly of titanium dioxide. EDX analysis of the materials was the same as present in figure $6.1 \mathrm{~b}$.

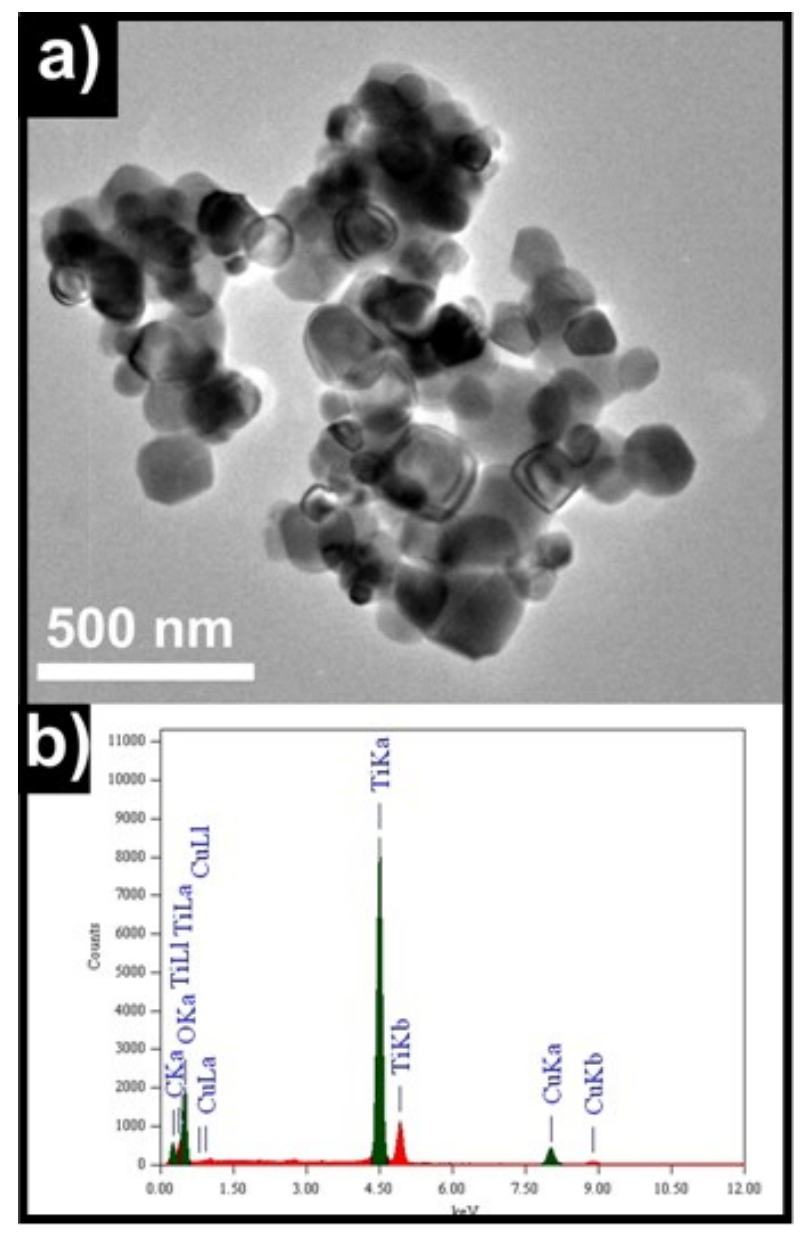

Figure 6.1: (a) a TEM micrograph of titanium dioxide obtained in experiment 1 (b) EDX spectrum of titanium dioxide obtained from experiment 2. 
Experiments 1 and 2 showed that no titanium disulphide was formed by reacting titanium isopropoxide and sulphur under these synthetic conditions. The absence of sulphur in the EDX analysis showed that that no reaction occurred between sulphur and the titanium source. The mechanism by which amorphous titanium dioxide formed from titanium isopropoxide is unclear, but the liberation of water either from the precursor or from the reaction of the precursor with the sulphur solution is thought to be a reason for the formation of titanium dioxide. 


\subsection{The reaction of Titanium Tetrachloride and Sulphur in 1-Octadecene (Expt. 3)}

An experiment was carried out reacting elemental sulphur and titanium tetrachloride in 1-Octadecne. The reactants were mixed together at room temperature and the temperature of the reaction mixture slowly increased to $250^{\circ} \mathrm{C}$, and reacted at that temperature for $2 \mathrm{~h}$. Crystalline bulk titanium disulphide was obtained in this experiment. As can be seen in the low resolution TEM image in figure 6.2 (a), large particles of size ranging from $500 \mathrm{~nm}$ to a few microns were obtained. The large particles were found to have smaller clusters of $\mathrm{TiS}_{2}$ nanocrystals (figure 6.2b) in their periphery. Figure $6.2 \mathrm{c}$ is a high resolution TEM image of a section of such a cluster. The clusters consisted of crystalline $\mathrm{TiS}_{2}$ nanocrystals and clearly observable lattice fringes as seen in the figure. An SAED pattern of the clusters indicated that they were highly crystalline and corresponded to the characteristic hexagonal phase of $\mathrm{TiS}_{2}$ (Figure $6.3 \mathrm{~b}$ ). An EDX analysis showed that the as-synthesized materials were made up of $\mathrm{TiS}_{2}$ in a stoichiometric 1:2 ratio (Figure 6.3a). The presence of bulk titanium disulphide in the sample proved that crystalline and stoichiometric $\mathrm{TiS}_{2}$ could be synthesized using a colloidal route. 

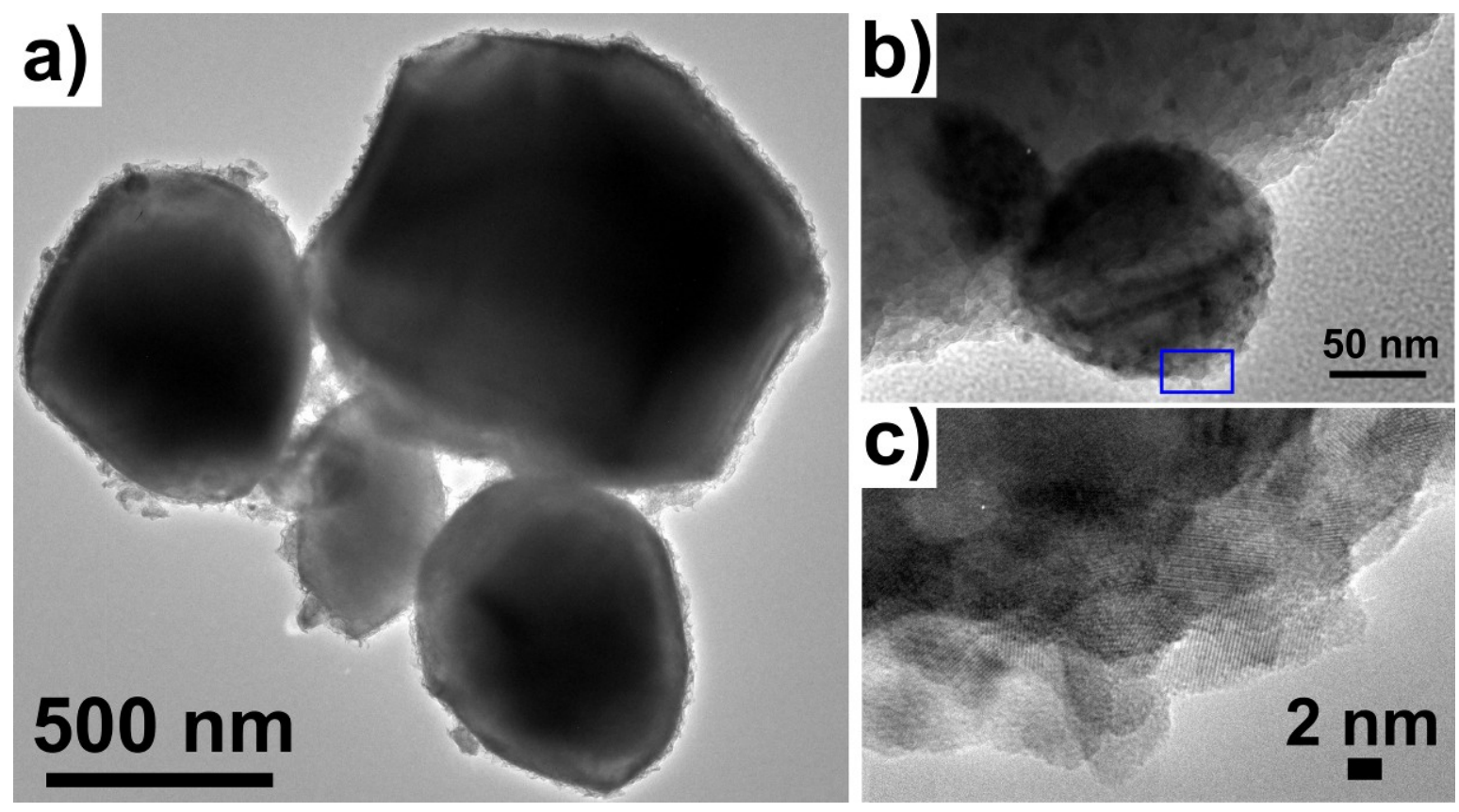

Figure 6.2: (a) low magnification TEM image of a cluster of bulk TiS2 particles, (b) TEM image of a cluster of $\mathrm{TiS}_{2}$ particles at the periphery of a bulk particle and (c) HRTEM image of the rectangular section in (b)

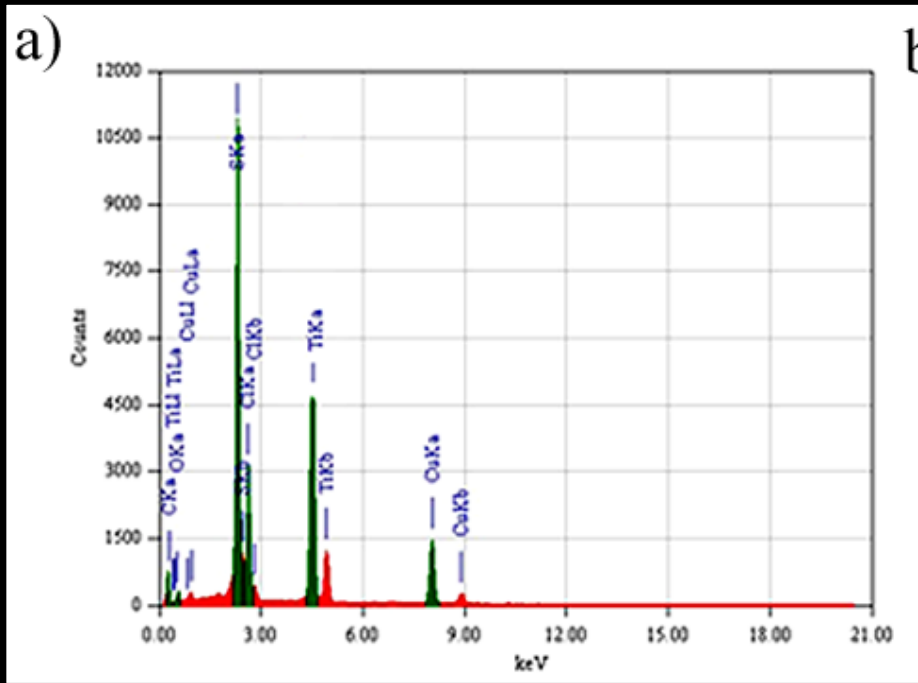

b)

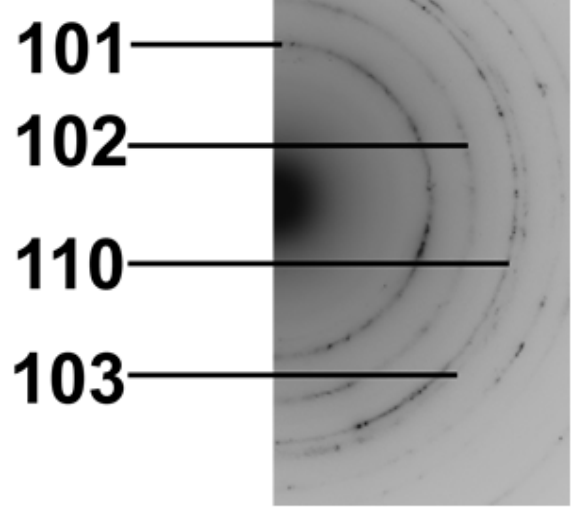

Figure 6.3: (a) EDX analysis of bulk TiS2 and (b) SAED pattern of the $\mathrm{TiS}_{2}$ clusters 


\subsubsection{The Reaction of Titanium Tetrachloride and Sulphur in 1-Octadecene varying precursor injection temperature (Expt. 4 and 5)}

An experiment was carried out reacting elemental sulfur and titanium tetrachloride in 1Octadecene. Experiment 4 had both an injection and a reaction temperature of $300^{\circ} \mathrm{C}$ of the titanium tetrachloride in the elemental sulfur solution for $10 \mathrm{~min}$. Low and medium magnification images of the as-synthesized nanostructured materials formed are shown in figure 6.4.

From the images it may be observed that the structures are three-dimensional, partly spherical, and flower-like in appearance. Figure 6.4(a) is a typical SEM image and shows the flower-like nanostructures aggregated together. The flower-like nanostructures tend to cluster in groups of around 10-50. Individual flower-like nanostructures are typically between 100 and $500 \mathrm{~nm}$ in size. Higher magnification images showing more detailed morphology of the flower-like nanostructures are shown in figure 6.4(b) and indicate that the flower-like nanostructures are built from tens of nanopetals. As can be seen in figure 6.4(c), individual flowers are made up of randomly aligned nanopetals of about $100-500 \mathrm{~nm}$ in length and $20-50 \mathrm{~nm}$ in width. Sonication of the nanostructures did not break them into discrete petals, suggesting that the nanostructures are integrated and not an aggregation of nanopetals. 

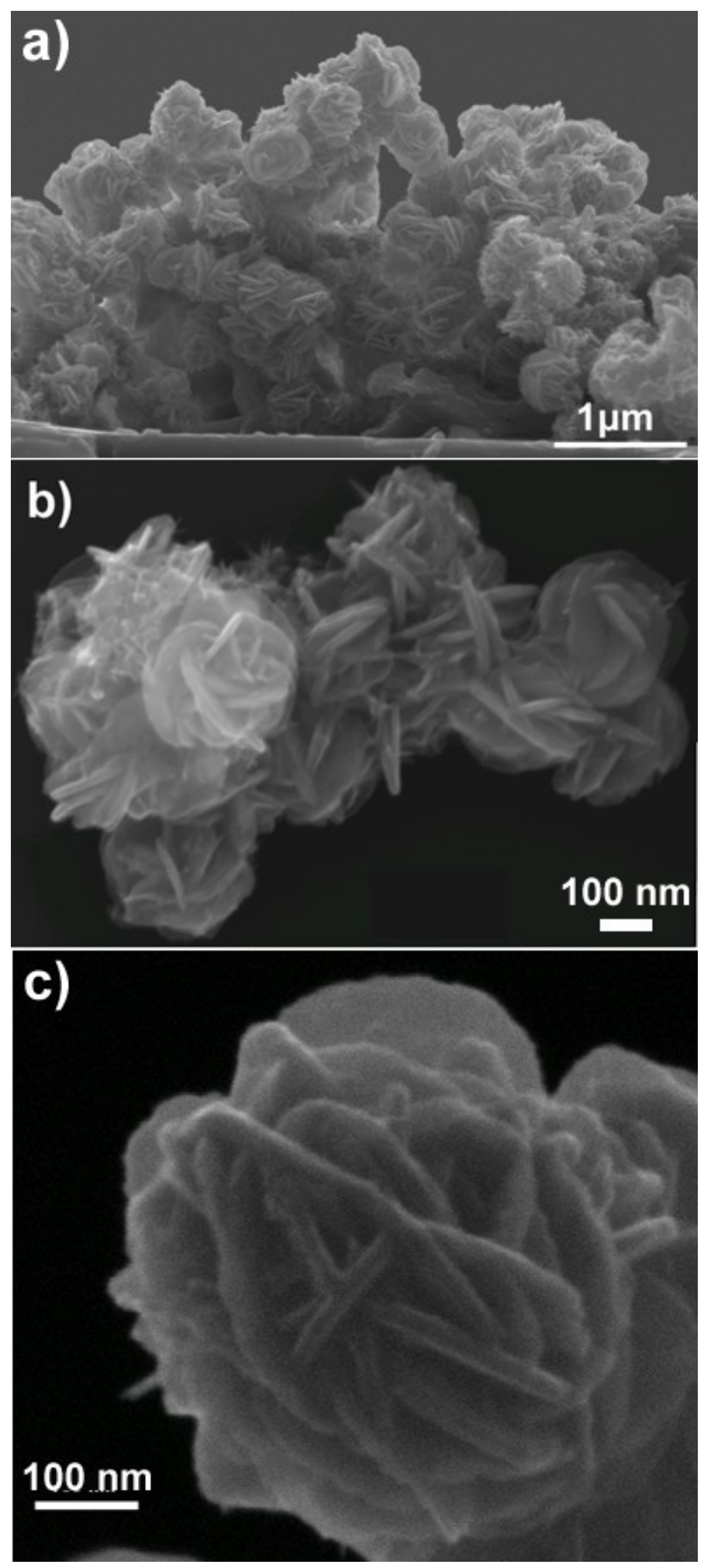

Figure 6.4: (a) SEM images (a) and (b) are low-magnification images of flower-like TiS2 nanostructures and (c) individual flower-like nanostructures. 
Further insight into the morphology and structure of the flower-like nanostructures was gained using low- and high-resolution transmission electron microscopy (TEM). The TEM images in parts (a) and (b) of figure 6.5 shows aggregates of flower-like nanostructures and an individual flower-like nanostructure, respectively. From the images it may be seen that the petals are about $25 \mathrm{~nm}$ in width and taper towards the edge.

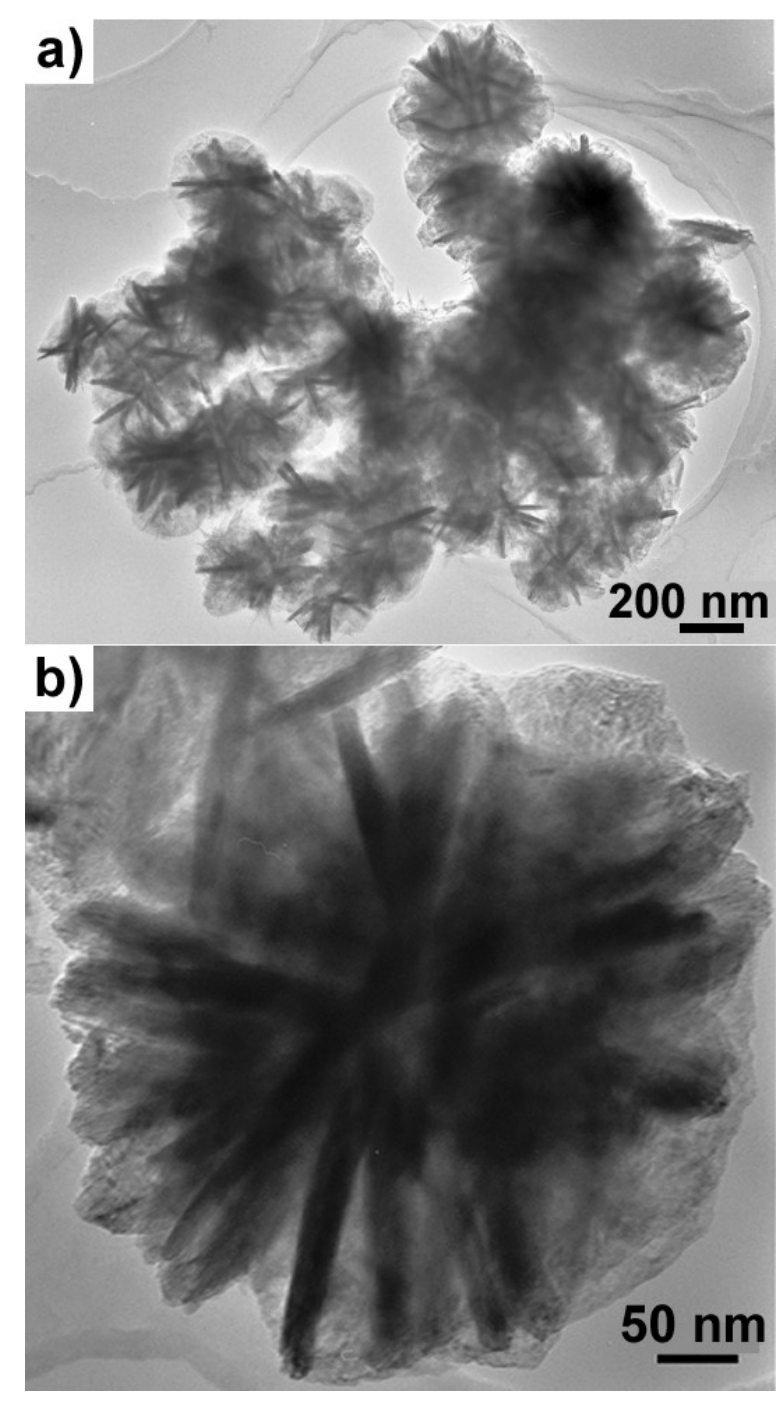

Figure 6.5: (a) Low-resolution TEM image of as-prepared $\mathrm{TiS}_{2}$ nanoflowers; (b) a single nanoflower exhibiting rod-like growth originating from the centre of the flake. 
The nanopetals are crystalline and the TEM image in figure 6.6 shows clearly observable lattice fringes. Fast Fourier transform (FFT) analysis (inset) of the corresponding image matched the crystal structure of titanium disulphide viewed down the [001] orientation.

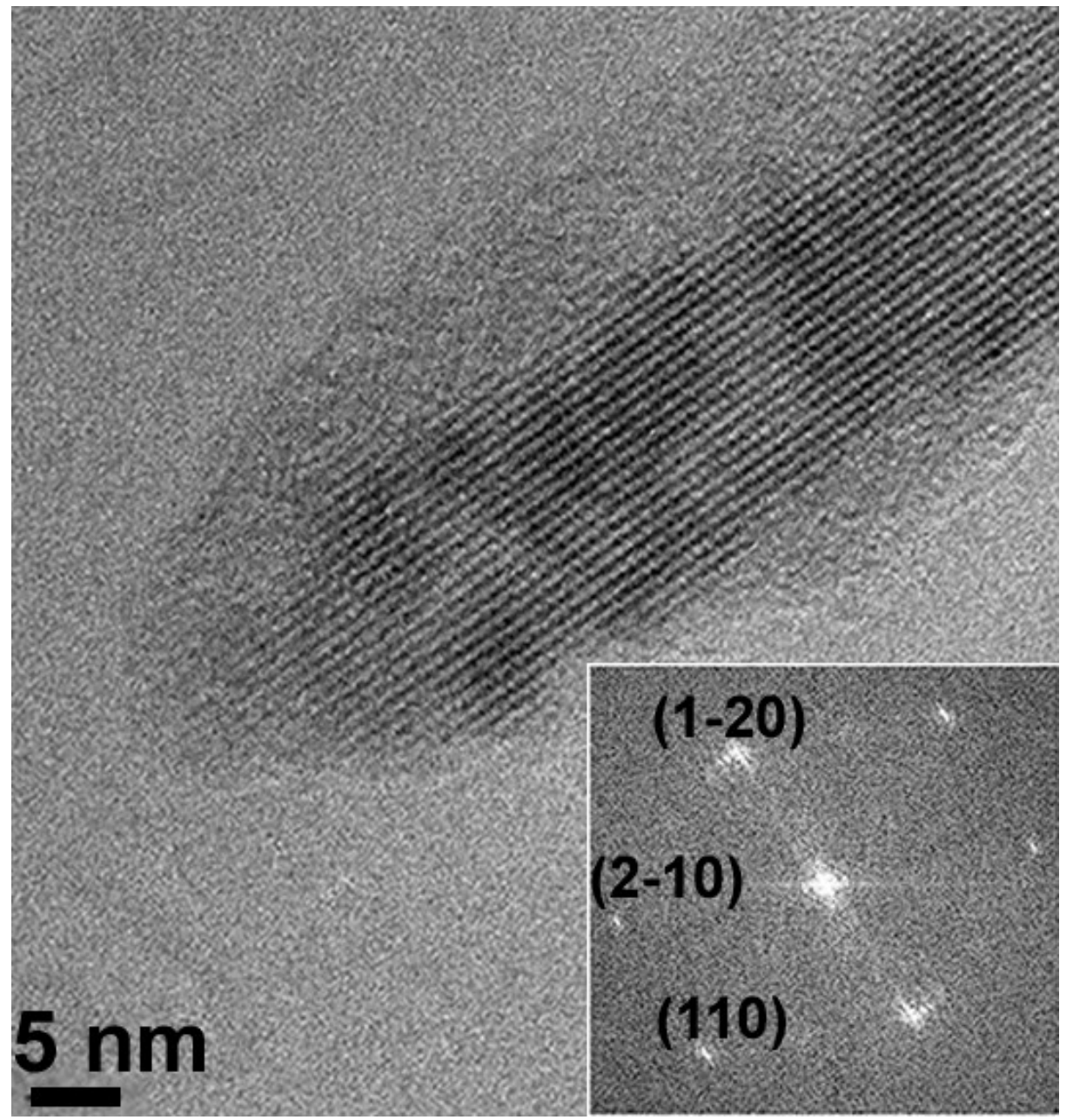

Figure 6.6: High-resolution TEM image of a nanopetal and (inset) FFT pattern of the corresponding image.

An X-ray powder diffraction pattern of the TiS2 flower-like nanostructure is shown in figure 6.7. The reflections could be readily indexed to the hexagonal phase of $\mathrm{TiS}_{2}{ }^{4}$ 


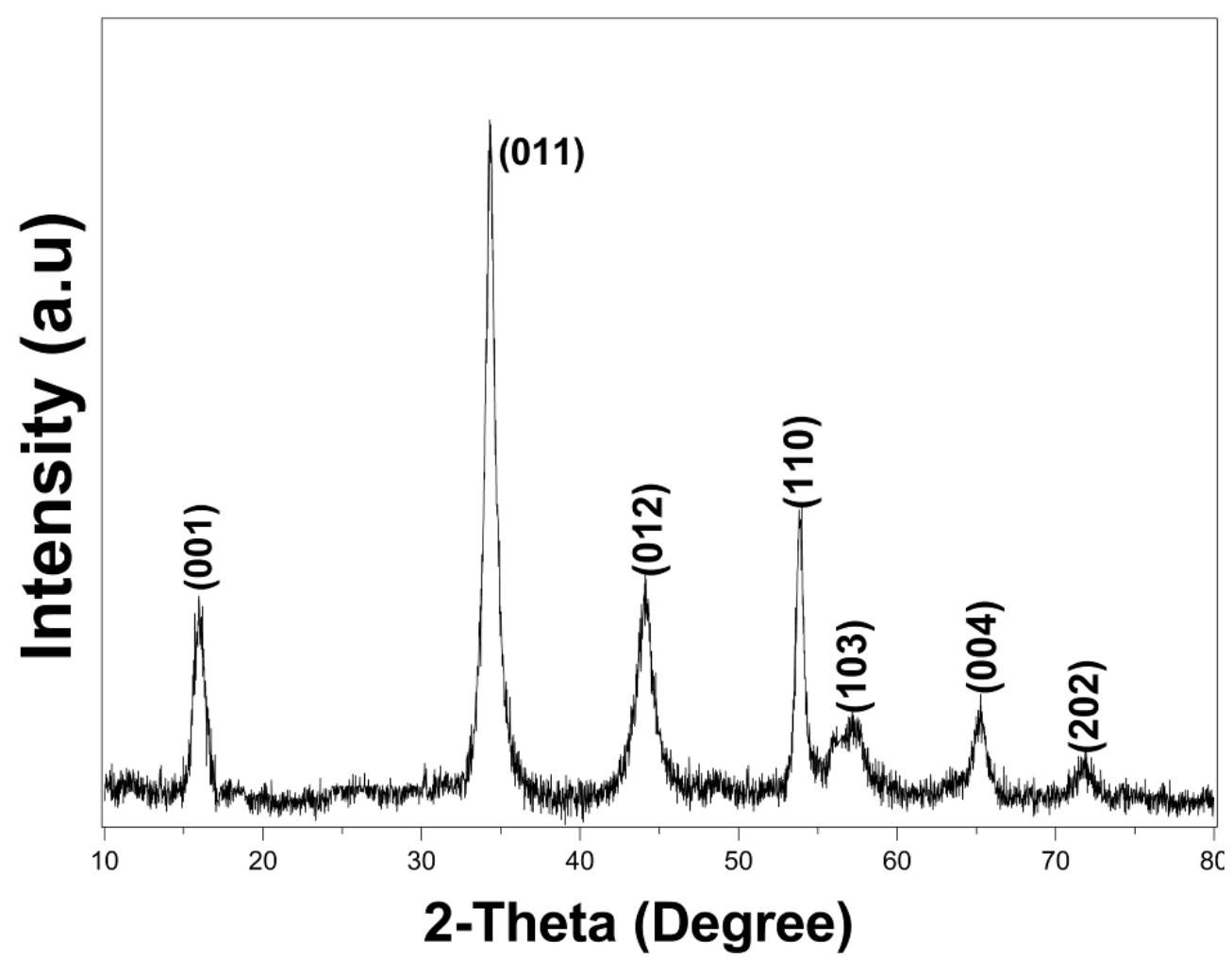

Figure 6.7: XRD pattern of the $\mathrm{TiS}_{2}$ flower-like nanostructure.

In experiment 5 the injection temperature of the titanium source into the elemental sulfur solution was decreased to $150^{\circ} \mathrm{C}$. After injection at $150^{\circ} \mathrm{C}$, the temperature of the solution was gradually increased to $300^{\circ} \mathrm{C}$ over a period of $15 \mathrm{~min}$ and the reaction further heated to $10 \mathrm{~min}$. The as-synthesized product was purified and dispersed in hexane for analysis by SEM and TEM. SEM and TEM images of the products are shown in figure 6.8. As can be seen from figure 6.8(a), the product was highly irregularly shaped ${ }_{\text {TiS2 }}$ fragment, which are referred to as flake-like nanostructures that were typically tens of micrometers in size. The higher magnification SEM image shown in figure $6.8(\mathrm{~b})$ shows that each of these flake-like nanostructures were made of smaller 
flakes about a micrometer in size attached to each other in random orientations to form discrete flake-like nanostructures and do not disintegrate upon sonication.
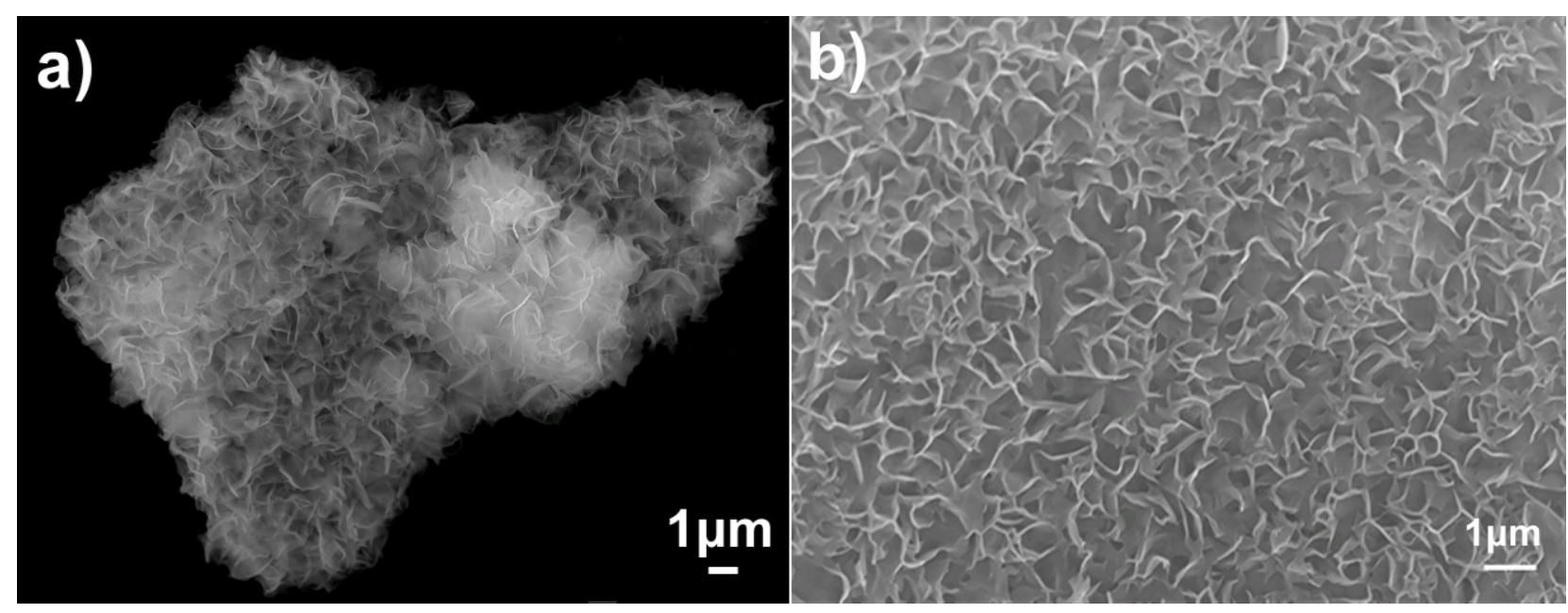

Figure 6.8: Low-resolution SEM image of the flake-like nanostructures; (b) higher magnification view of the flakes.

Figure 6.9 shows an individual flake-like nanostructure of $\mathrm{TiS}_{2}$, showing good agreement with SEM images, and illustrates that the nanostructures are made up of flaky protrusions. The light contrast in many areas of the TEM image in figure 6.9 indicates the thin 2D nature of the flake-like nanostructures. 


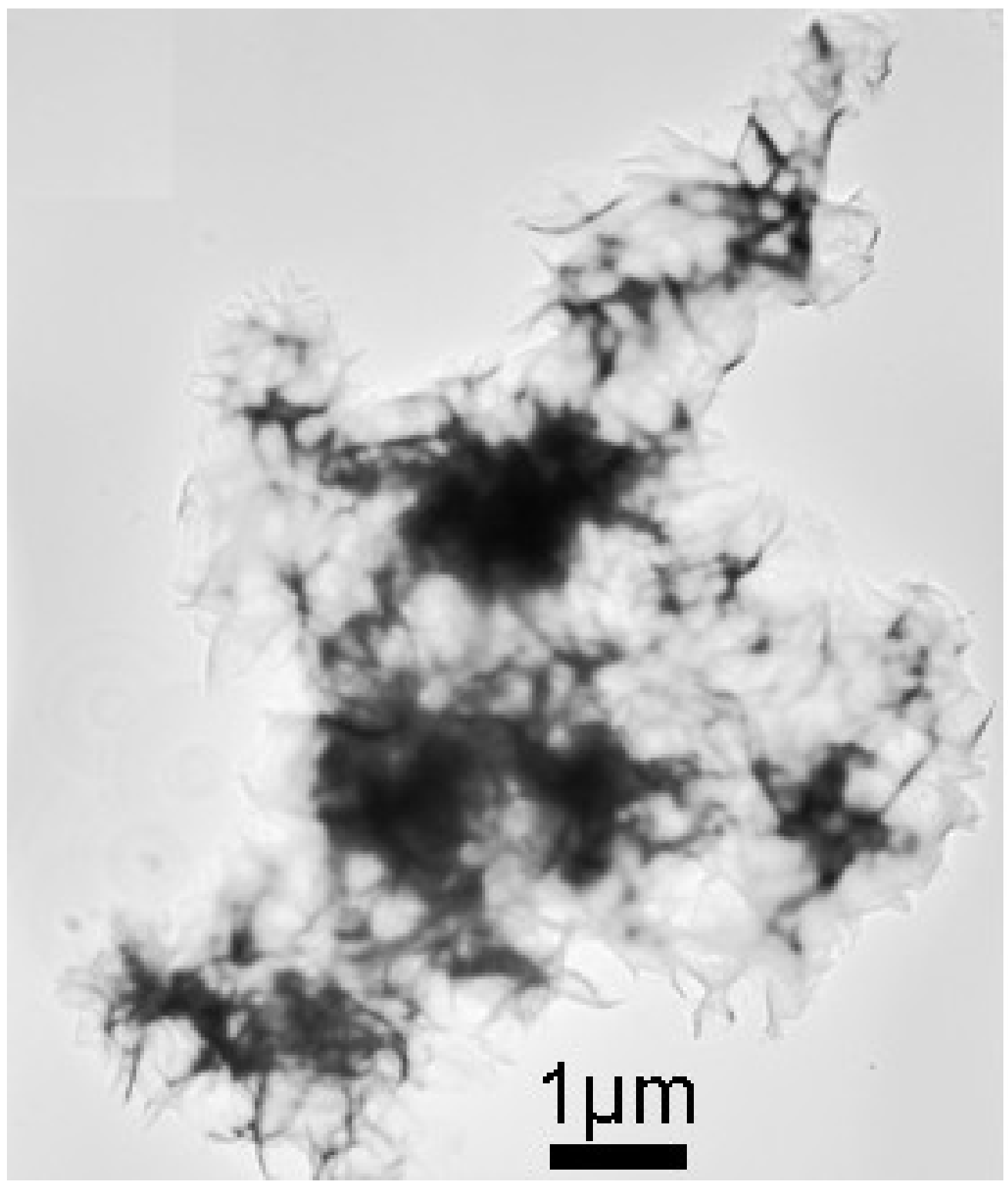

Figure 6.9: $\mathrm{TEM}$ image of a single $\mathrm{TiS}_{2}$ flake.

The flake-like nanostructures were found to be crystalline and SAED patterns could be indexed to the hexagonal phase of $\mathrm{TiS}_{2}$. EDX analysis was carried out on individual flakes of $\mathrm{TiS}_{2}$, which showed good agreement with 1:2 molar ratios of titanium to sulphur. 


\subsubsection{Variation of Reaction Time and Growth Mechanism}

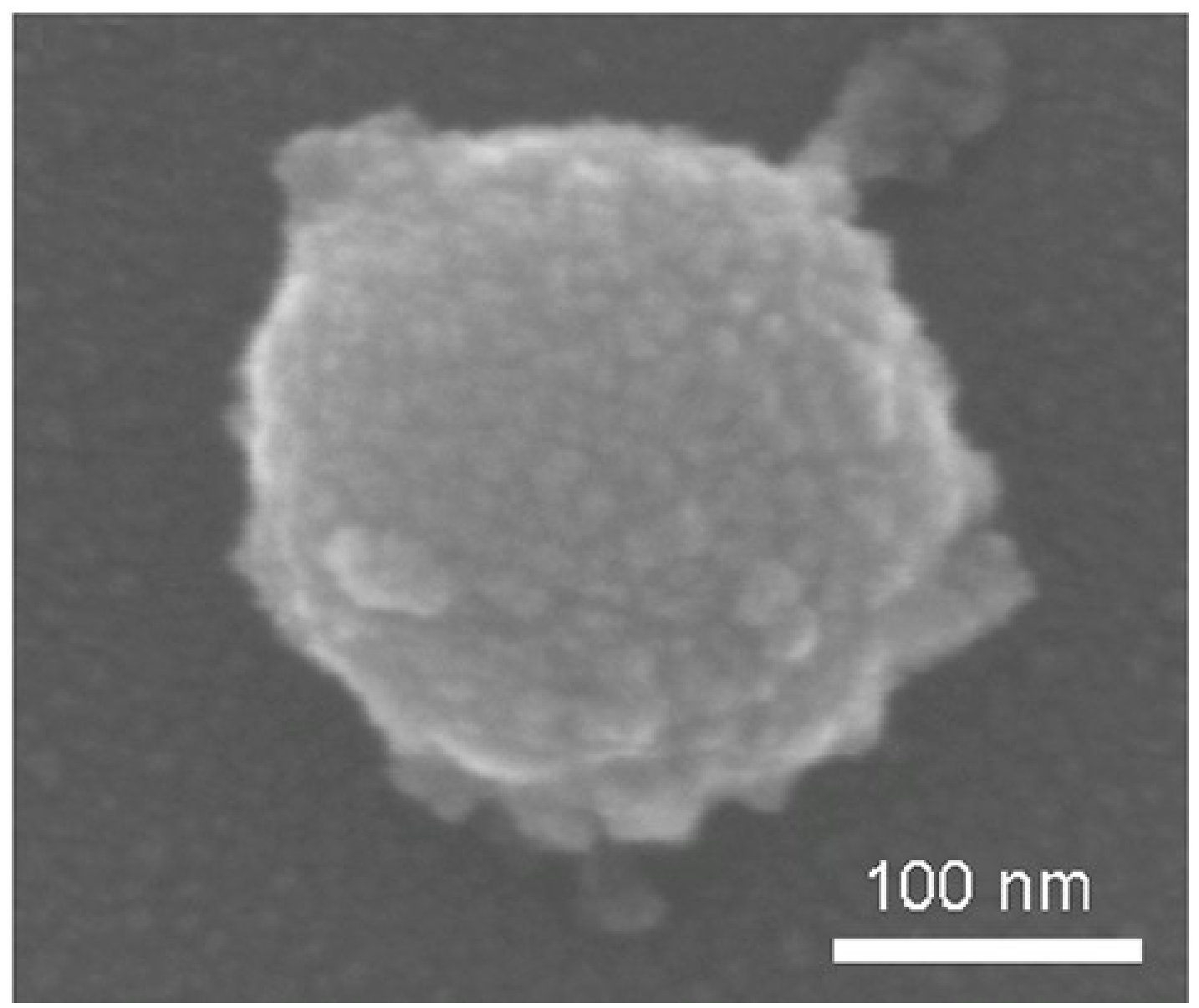

Figure 6.10: SEM image of the product obtained at $30 \mathrm{~s}$.

To investigate the growth mechanism of the flower-like $\mathrm{TiS}_{2}$, experiments were performed at $300^{\circ} \mathrm{C}$ where small aliquots were removed from the reaction flask at times of 30, 60, and $90 \mathrm{~s}$ and quenched in an ice bath. The SEM images shown in figures $6.12 \mathrm{a}-\mathrm{c}$ are of the samples obtained at these different reaction times and illustrate the evolution of the morphology of the $\mathrm{TiS}_{2}$ flower-like nanostructure. The sample taken after $30 \mathrm{~s}$ reaction time is shown in the image figure 6.10 . The products consist of a spherical core 
of size 200-300 nm with blisters growing from this core. These structures are defined as spheres with surface blisters. The surface blisters are roughly semi-spherical in shape with diameters typically less than $35 \mathrm{~nm}$. Energy-dispersive X-ray (EDX) analysis (figure 6.11) in TEM of the spheres with surface blisters showed that their composition was $\operatorname{TiS}_{1.3} \mathrm{Cl}_{0.8}$.

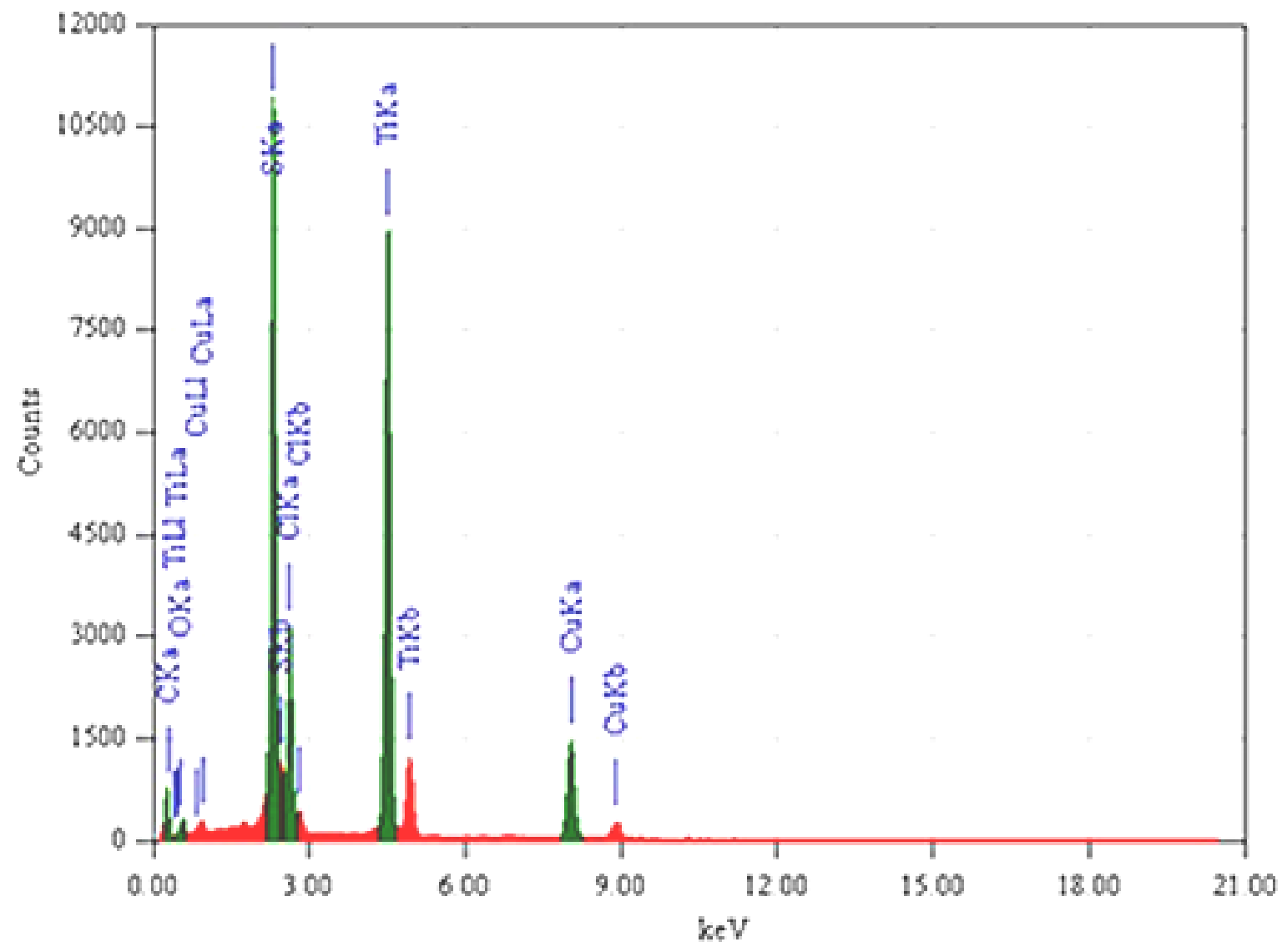

Figure 6.11: EDX spectrum of the product obtained at $30 \mathrm{~s}$

An SEM image of a typical particle from the sample with reaction time of $60 \mathrm{~s}$ is shown in figure $6.12(a)$. From SEM analysis its is found that over $80 \%$ of the product were particles similar to the one shown in figure 6.12 a, which were called spheres with 
protuberances and $20 \%$ of the product still remained as spheres with surface blisters. As shown in figure 6.12(a), a typical sphere with protuberances has a spherical core of similar size and structure to the spheres with surface blisters. As can be seen in figure 6.12(a), the protuberances are made of platelets that are randomly oriented, growing off the spherical core. From high-resolution TEM it was discovered that they are 10-100 atomic layers in thickness and typically $35-200 \mathrm{~nm}$ horizontally. TEM studies on both surface blisters and protuberances showed that they were crystalline and were composed of stoichiometric $\mathrm{TiS}_{2}$. However, from selected area electron diffraction (SAED), the spherical core was found to be amorphous.
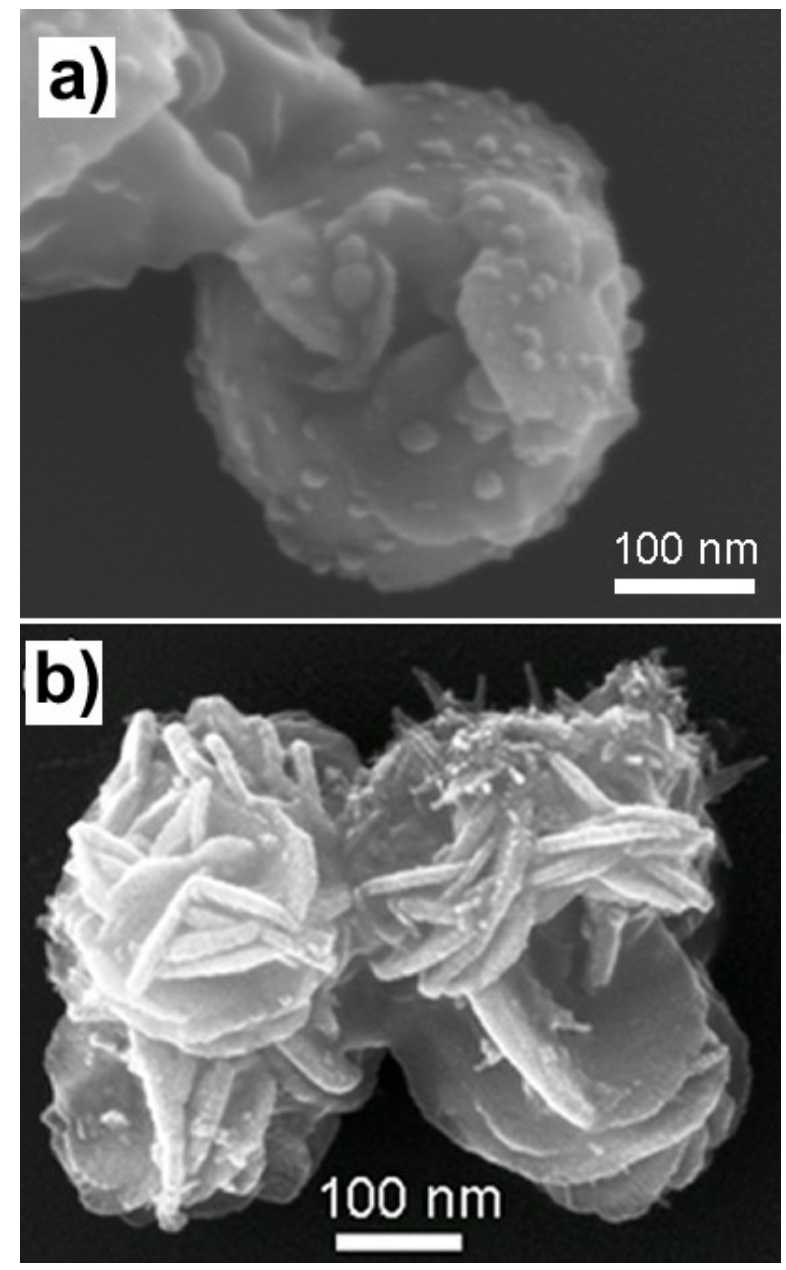

Figure 6.12: SEM images of the products obtained at $60 \mathrm{~s}$ and $90 \mathrm{~s}$ 
At $90 \mathrm{~s}$, the sample contained flower-like nanostructures of $\mathrm{TiS}_{2}$ in a $100 \%$ yield. A typical flower-like nanostructure is shown in figure $6.12 \mathrm{~b}$. As can be seen from figure $6.12 \mathrm{~b}$, these structures are made up of randomly aligned nanopetals originating from the centre that are so large that the spherical core is no longer evident. SAED indicated that the flower-like nanostructures are highly crystalline. Reactions for longer time periods were also found to form identical flower-like nanostructures, suggesting that they are the final thermodynamic product. EDX analysis in TEM of the final flower-like nanostructures indicated a composition of $\mathrm{TiS}_{1.8}$.

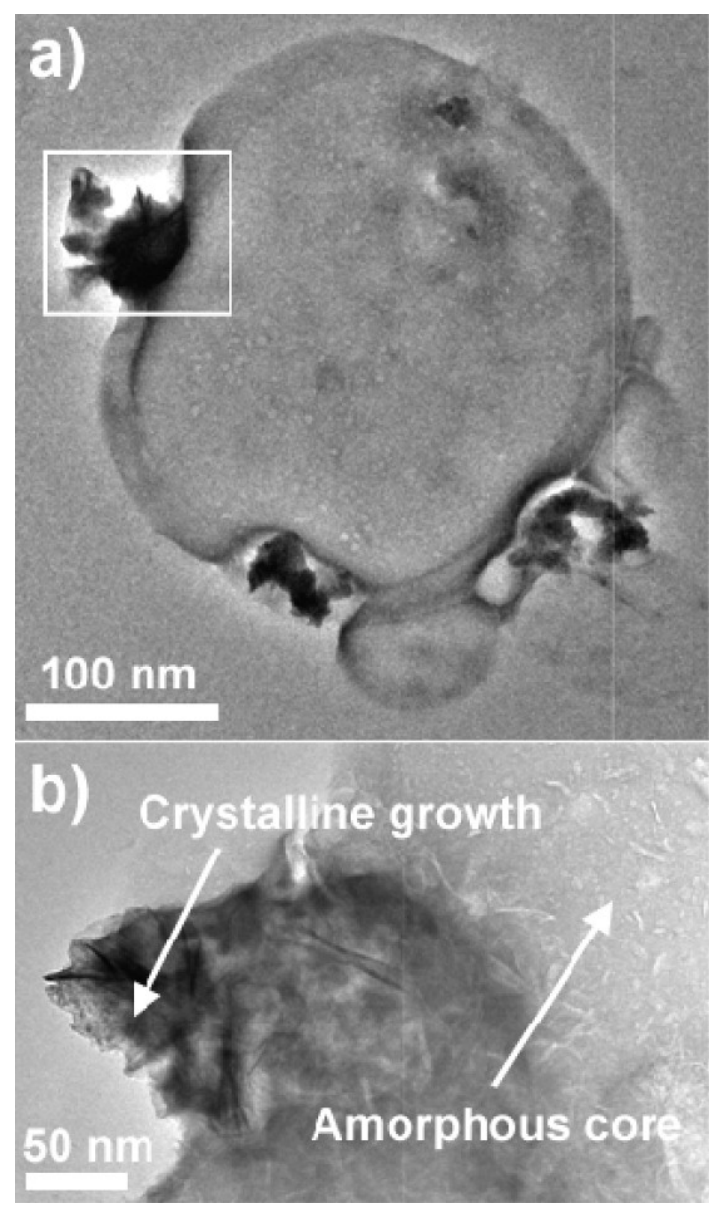

Figure 6.13: (a) Low-magnification TEM image of a sphere isolated at $60 \mathrm{~s}$. (b) Higher magnification image of the rectangular section in Figure 6.13(a), indicating the crystalline and amorphous part of the sphere. 
TEM analysis of the spheres with surface blisters was undertaken to further characterize the intermediate structure. The TEM image of a typical sphere with surface blisters isolated at $60 \mathrm{~s}$ is shown in figure 6.13 and a higher magnification image in figure 6.13b. SAED of the area boxed in white containing the surface blister indicated that the surface blister was crystalline and SAED of the spherical core gave an amorphous diffraction pattern.

To understand the formation of the flake-like nanostructures, the reaction was repeated with small aliquots removed at different reaction times in a fashion similar to the method used for the flower-like nanostructures. The product in all the aliquots removed were analyzed by SEM and TEM and even those taken after only $1 \mathrm{~s}$ were flake-like nanostructures with the same size and morphology of those found at the longer reaction times and shown in figure 6.9. 


\subsubsection{BET Surface Area Measurements on Nanostructured Flower-like and Flake-like Titanium Disulphide}
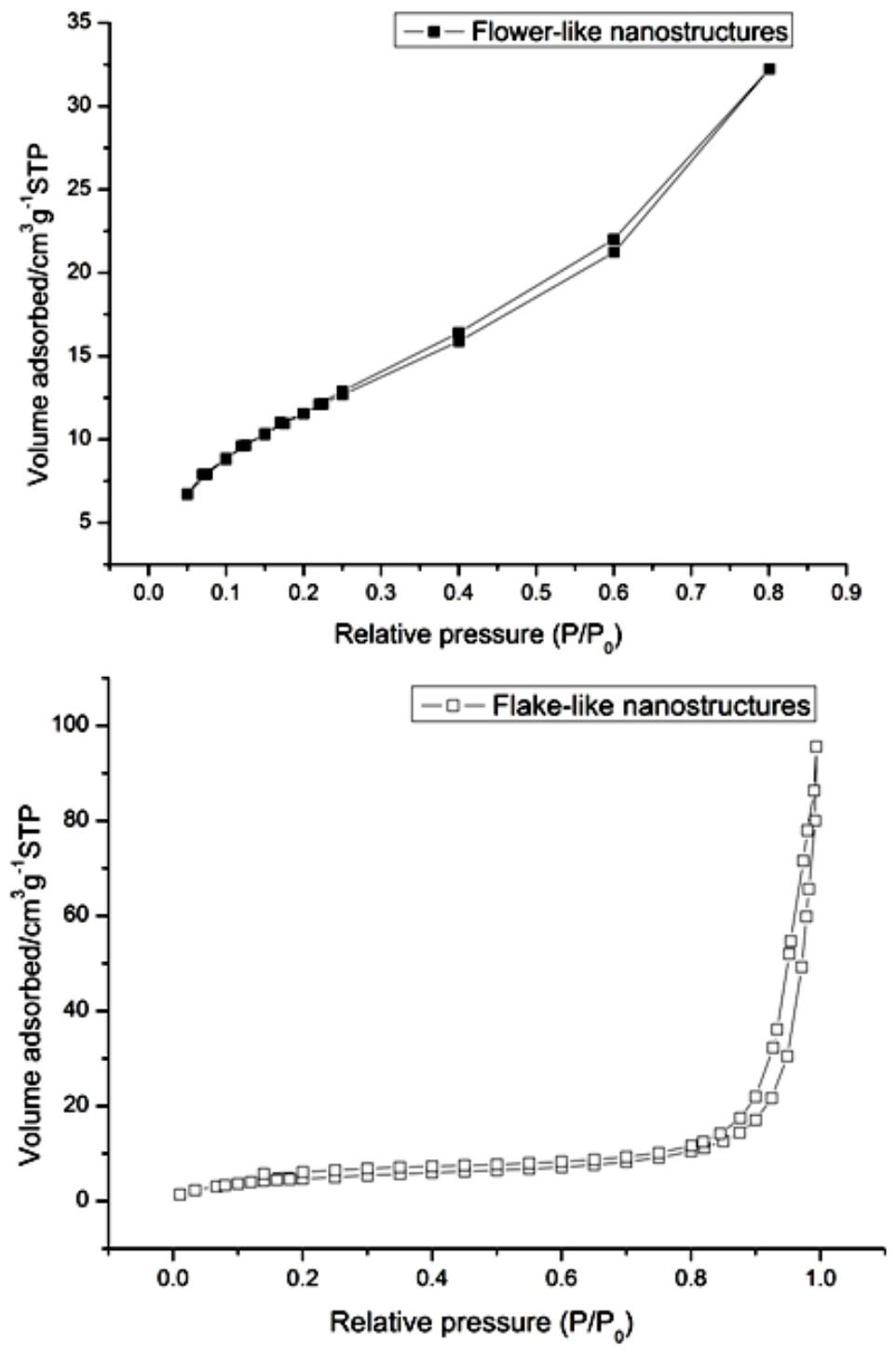

Figure 6.14: BET isotherms of flower-like and flake-like nanostructures.

Titanium disulphide is known to have possible applications as hydrogenation catalysts and hydrogen storage devices. For these applications high surface area is important. 
BET surface area measurements were conducted on both flower-like and flake-like nanostructures and were found to be 32 and $19 \mathrm{~m}^{2} \mathrm{~g}^{-1}$, respectively as can be seen in figure 6.14. 


\subsection{The Reaction of Titanium Tetrachloride and Elemental Sulfur in 1-Octadecene with Oleylamine as Surfactant (Expt. 6 and 7)}

An experiment was carried out by reacting titanium tetrachloride and elemental sulphur in 1-Octadecene with oleylamine as surfactant. The titanium source was injected at both high (experiment 6) and low temperatures (experiment 7) of the elemental sulphur solution. However, the products obtained were similar to that obtained for the reaction involving just $\mathrm{TiCl}_{4}$ and elemental sulphur in 1-Octadecene (Experiment 4 and 5). SAED analysis of the products obtained for both high and low temperature injection revealed that flower-like and flake-like nanostructures were obtained, with no reduction in size or change in morphology of the nanostructures. 


\subsection{The Reaction of Titanium Tetrachloride and Elemental Sulfur in Oleylamine (Expt. 8 and 9)}

An experiment was carried out by reacting titanium tetrachloride and elemental sulphur in oleylamine as the solvent and surfactant. The titanium source was injected at both high and low temperatures of the elemental sulphur solution. Experiment 8 had both an injection and reaction temperatures of $250^{\circ} \mathrm{C}$ of the titanium tetrachloride in the elemental sulphur solution for 90 mins. Medium magnification and high resolution TEM images of the nanostructures materials formed are shown in figure 6.61 (a) and (b).

Fullerene-like nanoparticles of titanium disulphide were obtained. From the images in figure 6.15 it may be observed that the structures are a mixture of spherical or faceted fullerene-like structures with a minor amount of nanotubes. The fullerene-like nanoparticles tend to cluster in groups of around 10-25, with individual nanoparticles typically 20-30 nm in size. Higher magnification TEM images showing more detailed morphology of the fullerene-like nanostructures are shown in figures 6.15(a) and (c) and indicate that the fullerene-like nanostructures depending on their sizes were made up of 20-30 concentric and spherical layers. Each of these dark lines that make up the concentric circles of the nanoparticle represents an atomic layer of the basal plane (001). At the centre of these fullerene-like nanoparticles lies a hollow core and again depending on the size of the nanoparticles ranged from 5-8 $\mathrm{nm}$ (figure 6.15). The fullerene-like nanoparticles were crystalline and the TEM image in figure 6.15(a) shows clearly observable lattice fringes. An SAED pattern of a cluster of fullerene-like nanoparticles of $\mathrm{TiS}_{2}$ is shown in figure 6.16(a). 


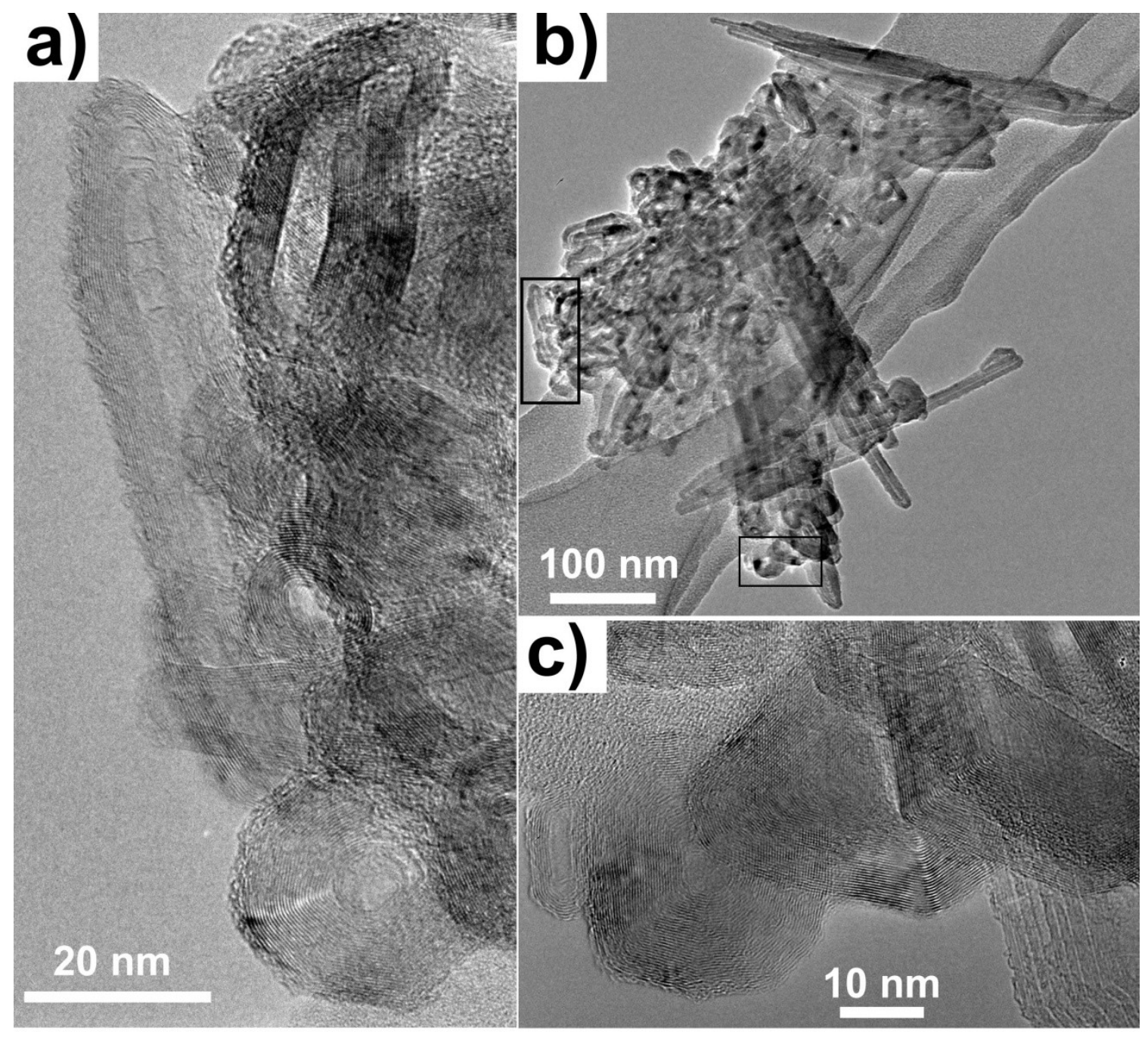

Figure 6.15: (a) TEM image of a cluster of fullerene-like nanoparticles and (b) HRTEM image of a fullerene-like nanoparticle.

EDX analysis of the clusters show a 1:2 ratio of titanium to sulphur as can be seen in figure $6.16(b)$. 


\section{a)}

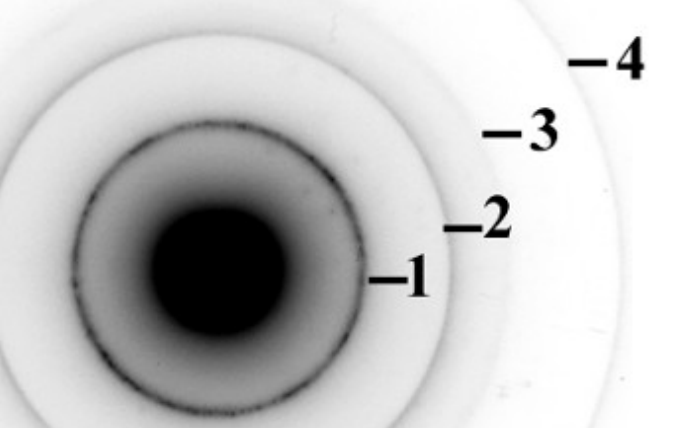

\section{b)}

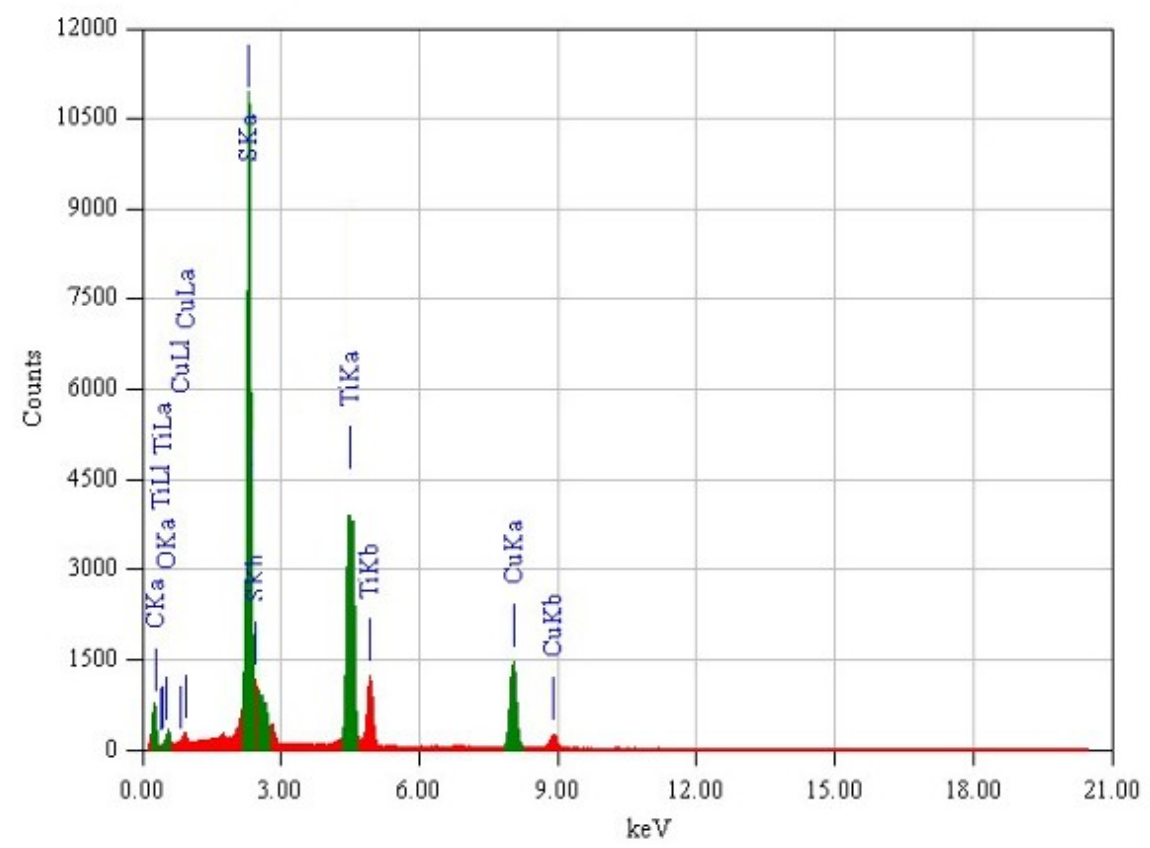

Figure 6.16(a): SAED pattern of a cluster of fullerene-like nanoparticles, rings matched the crystal structure of TiS2: ring $1=(001), 2=(102), 3=(110)$ and $4=(111)$ and $(b)$ EDX of the corresponding cluster. 

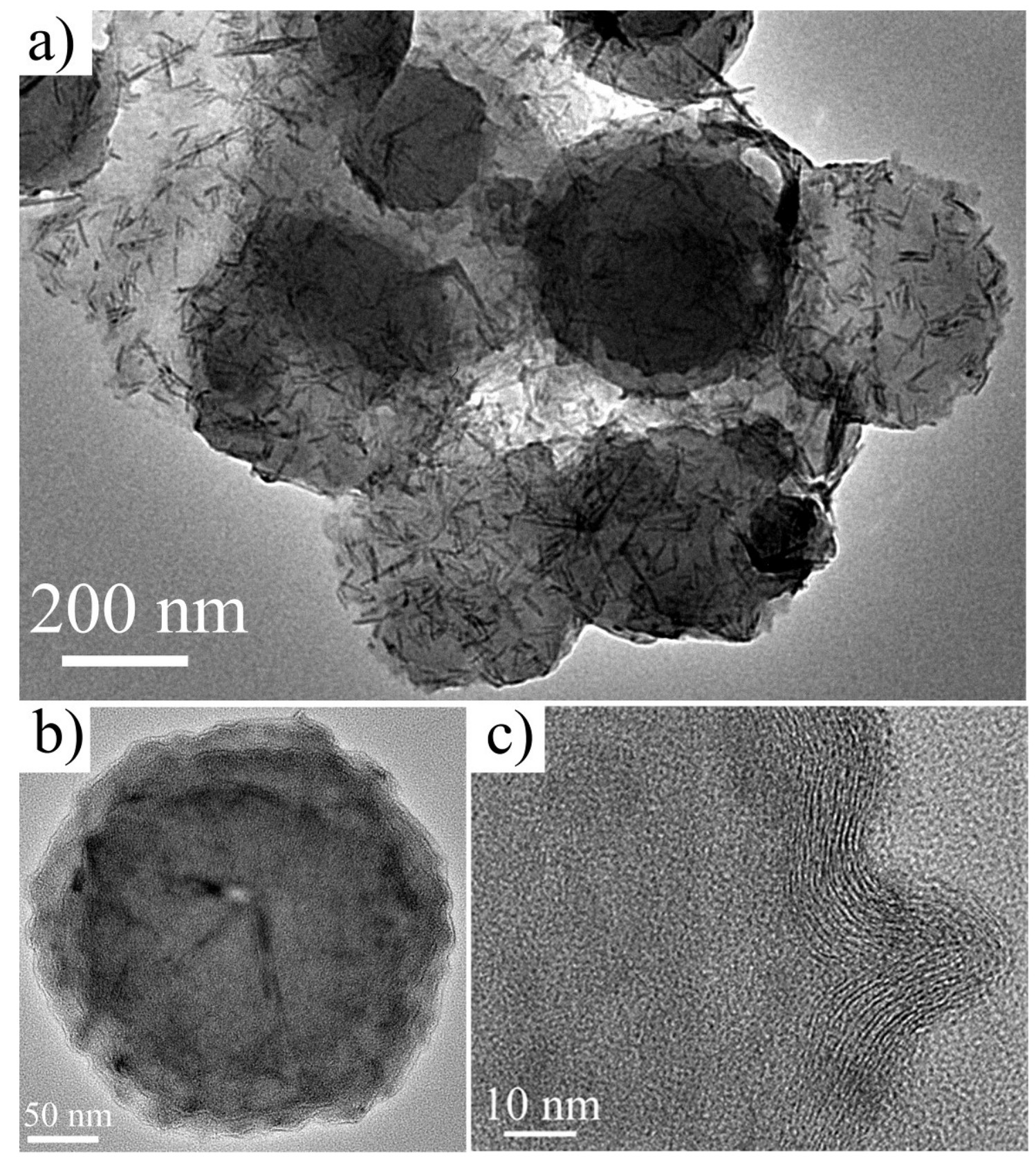

Figure 6.17(a): low magnification TEM image of aggregates of hollow $\mathrm{TiS}_{2}$ spheres,

(b) TEM image of an individual hollow sphere and (c) HRTEM image of the external surface of a hollow sphere. 
In experiment 9 the titanium source was injected into the sulphur containing oleylamine solution at room temperature. After injection the temperature of the reaction mixture was rapidly raised to $250^{\circ} \mathrm{C}$ and reacted at that temperature for 90 minutes. Hollow spherical spheres of $\mathrm{TiS}_{2}$ (figure 6.17a) were obtained.

The hollow spheres were $300-450 \mathrm{~nm}$ in size and were found in aggregates of about 1020 spheres. Figure 6.17(b) is a TEM image of an individual hollow sphere. The higher magnification TEM image of such a sphere is shown in figure 6.17(c) and shows the presence of disordered lattice fringes. The external surface of the hollow spheres is rough and a high concentration of surface defects can be seen. The EDX analysis of the hollow sphere showed a 1:2 ratio of Ti: S. 


\subsection{Discussion}

\subsubsection{Chemical Reaction}

The formation of crystalline $\mathrm{TiS}_{2}$ from $\mathrm{TiCl}_{4}$ and elemental sulphur most likely occurs through the overall reaction shown in the equation below. Sulphur has been known to act as both the reductant and sulfiding agent at high temperatures. ${ }^{5-8}$ Hence a 1:6 ratio of Ti to $\mathrm{S}$ ensures complete sulfurisation.

$$
\mathrm{TiCl}_{4}+6 \mathrm{~S} \rightarrow \mathrm{TiS}_{2}+2 \mathrm{~S}_{2} \mathrm{Cl}_{2}
$$

\subsubsection{Growth Mechanism for the Formation of Flower-like and Flake-like Nanostructures}

On the basis of the electron microscopy results shown in figures 4 and 5, the growth mechanism for the formation of the flower-like nanostructures was elucidated and is depicted schematically in figure 6.18 .
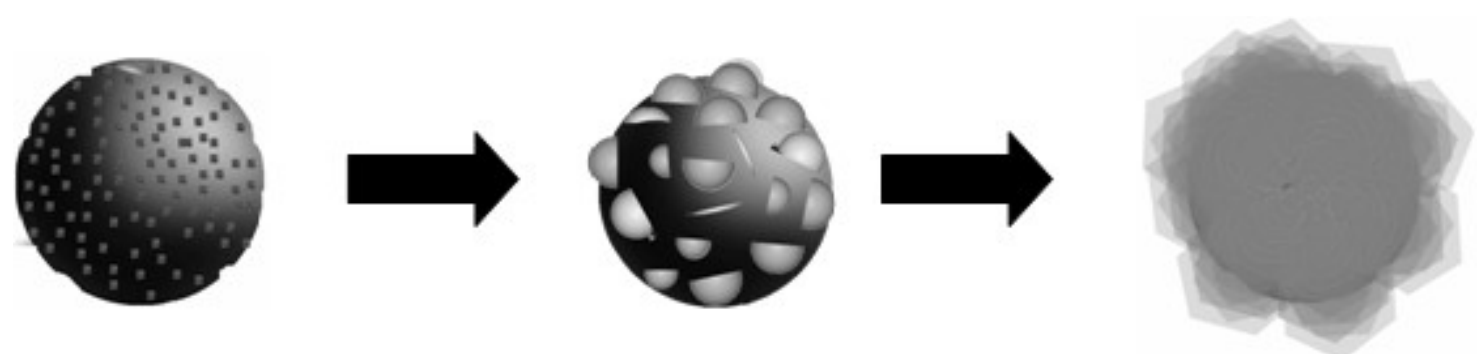

Figure 6.18: Growth Mechanism of the $\mathrm{TiS}_{2}$ flower-like nanostructures 
During the initial stages of nucleation, amorphous spheres between 200 and $300 \mathrm{~nm}$ with an approximate composition of $\mathrm{TiS}_{1.3} \mathrm{Cl}_{0.8}$ form. These spheres have small crystalline blisters of $\mathrm{TiS}_{2}$ on the surface to give spheres with surface blisters. As the reaction continues, the inner amorphous spherical core remains constant in size and the growth occurs on the surface of the spheres as the surface blisters grow into bigger protuberances. As the reaction continues to $90 \mathrm{~s}$, these protuberances have continued to grow into size to become full crystalline flower-like petals. These observations agree with previous reports of growth mechanism involving a multistage growth process. ${ }^{9-12}$

Thus, the growth and morphology of the flower-like nanostructures may be understood as follows. The initial particles formed are amorphous and thus adopt a spherical shape. The surface of the spheres, act as nucleation sites for the formation of smaller crystalline blisters of $\mathrm{TiS}_{2}$. As the reaction proceeds, it is energetically more favorable for the crystalline $\mathrm{TiS}_{2}$ blisters to grow, which they do via protuberances into full crystalline flower-like petals with the blisters on the surface of the spheres providing high-energy sites for crystalline growth. ${ }^{13,14}$ The plate-like 2D shape of the protuberances and flower-like petals originates from the layered structure of $\mathrm{TiS}_{2}$, which grows preferentially along the $a$ and $b$-axes compared to the $c$-axis.

The results obtained by varying reaction times for the synthesis of flake-like nanostructures show that the flake-like nanostructures are formed immediately after injection and prevented the isolation of initial products. The formation of $2 \mathrm{D}$ flake-like nanostructures in dichalcogenide systems originates from the layered crystal structure. Flake-like nanostructures form with growth along the [100] and [010] directions, these directions 
being more favorable because of stronger bonding than that seen from growth in the longer [001] direction. ${ }^{15}$

\subsubsection{Morphological Control}

The results show that the final morphology of the $\mathrm{TiS}_{2}$ nanostructure can be controlled by the nature of the initial nuclei. At a lower injection temperature of $150^{\circ} \mathrm{C}$ flake-like nanostructures are obtained, most likely from the instant nucleation of $\mathrm{TiS}_{2}$ flakes. At a higher injection temperature of $300^{\circ} \mathrm{C}$, flower-like nanostructures are obtained through the formation of spherical nuclei of $\mathrm{TiS}_{\mathrm{x}} \mathrm{Cl}_{\mathrm{y}}$. This understanding of the controlled and selected growth of both flower-like and flake-like nanostructures is highly significant for understanding the growth of all materials with similar layered structures including industrially important $\mathrm{MoS}_{2}, \mathrm{NbS}_{2}$ and $\mathrm{WS}_{2}$.

\subsubsection{Growth Mechanism for the Formation of Fullerene-like Nanoparticles}

The solvent/surfactant agent oleylamine plays an important role in the morphology of the final nanostructures. The spherical morphologies with relatively smooth curvature exhibited by these nanoparticles suggest that the bending of the molecular sheets of titanium disulphide into fullerene-like nanostructures could be due to the combination of these two main reasons. 1) The instability of the planar layered $\mathrm{TiS}_{2}$ structure which is formed immediately on injection of the titanium source into the hot sulphur-oleylamine solution; this leads to the shrinking size of the molecular sheet to the nano-regime which 
in turn leads to the increasing rim/bulk atoms ratio, making the planar nanosheets unstable and resulting in the folding of the layers into fullerene-like nanoparticles. ${ }^{17} 2$ ) The ability of oleylamine to bind to the surface of planar sheets during growth, preventing further growth in the planar direction and causing them to form stable fullerene like nanostructures. 


\subsection{Summary}

A new method was successfully developed to synthesize highly crystalline titanium disulphide nanostructures in solution. Flower-like and flake-like nanostructures were produced by varying the injection temperature of the titanium source into the 1Octadecene/sulphur solution. Two different pathways were suggested for the formation of the final products; an instant nucleation to form $\mathrm{TiS}_{2}$ flakes and spherical nuclei to form flower-like nanostructures. The flower-like nanostructures were found to have higher BET surface area compared to the flake-like nanostructures and previously reported surface areas for analogous $\mathrm{TiS}_{2}$ nanostructures. Whilst using oleylamine as solvent, the low temperature injection yielded hollow spheres of $\mathrm{TiS}_{2}$ and the high temperature injection, fullerene-like nanoparticles of $\mathrm{TiS}_{2}$. The property of oleylamine to selectively bind to the nanostructure surface in conjunction with the effect of injection temperature was understood to be behind the growth of these nanostructures. 


\subsection{Experimental}

Synthesis of Flower-like and Flake-like Titanium Disulphide Nanostructures in 1Octadecene (Expt. 4 \&5): A solution of a mixture containing 0.192 g of sulphur and 20 $\mathrm{ml}$ of 1-Octadecene was degassed and then heated to either $150^{\circ} \mathrm{C}$ (experiment 4 ) or $300^{\circ} \mathrm{C}$ (experiment 5). $0.1 \mathrm{ml}$ of titanium tetrachloride was then injected swiftly into the hot sulfur solution. In the case of experiment 4 the temperature of the reaction upon injection of the titanium source, was rapidly raised to $300^{\circ} \mathrm{C}$ and allowed to react at that temperature for 10 mins under nitrogen. In experiment 5 the reaction mixture was allowed to react for 10 mins after injection of the titanium precursor at $300^{\circ} \mathrm{C}$. In both cases the mixtures were quenched after reacting for 10 mins.

\section{Purification}

The reaction mixture after being cooled to room temperature was transferred to a centrifuge tube and diluted with $30 \mathrm{ml}$ of toluene. The supernatant was repeatedly discarded after five $10 \mathrm{~min}$ centrifugation cycles, after which the centrifugate was repeatedly washed with $20 \mathrm{ml}$ methanol. The final black product was then collected in a round-bottomed flask, sonicated and re-dispersed in n-hexane for characterization.

Synthesis of Fullerene-like nanoparticles and Hollow Spheres of Titanium Disulphide in Oleylamine (Expt. 8 \& 9): A solution of a mixture containing $0.192 \mathrm{~g}$ of sulfur, $20 \mathrm{ml}$ of oleylamine was degassed and then heated to $250^{\circ} \mathrm{C}$ (experiment 8 ) or kept at room temperature (experiment 9). $0.1 \mathrm{ml}$ of titanium tetrachloride was then 
injected swiftly into the sulfur solution. In the case of experiment 8 the temperature of the reaction upon injection of the titanium source, was rapidly raised to $300^{\circ} \mathrm{C}$ and allowed to react at that temperature for 90 mins under nitrogen. In experiment 9 the reaction mixture was allowed to react for 90 mins after injection of the titanium precursor at room temperature. In both cases the mixtures were quenched after reacting for 90 mins.

Purification: The reaction mixture after being cooled to room temperature was transferred to a centrifuge tube and diluted with $30 \mathrm{ml}$ of toluene. The supernatant was repeatedly discarded after five 10 min centrifugation cycles, after which the centrifugate was repeatedly washed with $20 \mathrm{ml}$ methanol. The final bluish-black product was then collected in a round-bottomed flask, sonicated and re-dispersed in n-hexane for characterization. 


\subsection{References}

1 Hahn, H.; Ness, P., Subchalcogenide phases of titanium, Z.Anorg. Allg. Chem., 1959, 302, 17.

2 Chen, J.; Li, S.-L.; Tao, Z.-L.; Shen, Y.-T.; Cui, C.-X., Titanium disulphide nanotubes as hydrogen storage devices, J. Am. Chem. Soc. 2003, 125, 5284.

3 Chen, J.; Tao, Z.-L.; Li, S.-L., Lithium intercalation in open-ended $\mathrm{TiS}_{2}$ nanotubes, Angew. Chem., Int. Ed. 2003, 42, 2147.

4 Natl. Bur. Stand. (U.S.) Monogr. 1966, 25 (4), 72.

5 Li, X. L.; Ge, J. P.; Li, Y.-D., Atmospheric pressure chemical vapour deposition: An alternative route to large-scale MoS2 and WS2 inorganic fullerene-like nanostructures and nanoflowers, Chem. Eur. J. 2004, 10, 6163.

6 Li, X. L.; Li, Y.-D., Formation of $\mathrm{MoS}_{2}$ inorganic fullerenes (IFs) by the reaction of $\mathrm{MoO}_{3}$ nanobelts and S, Chem. Eur. J. 2003, 9, 2726.

7 Ge, J. P.; Li, Y.-D., Controlled CVD route to CoS and MnS single-crystal nanowires, Chem. Commun. 2003, 19, 2498. 
8 Ge, J. P.; Li, Y.-D., Selective atmospheric pressure chemical vapor deposition route to CdS arrays, nanowires, and nanocombs, Adv. Funct. Mater. 2004, 14, 157.

9 Burda, C.; Chen, X. B.; Narayanan, R.; El-Sayed, M. A., Chemistry and properties of nanocrystals of different shapes, Chem. Rev. 2005, 105, 1025.

10 Cheng, Y.; Wang, Y. S.; Zheng, Y. H.; Qin, Y., Two-step self assembly of nanodisks into plate-built cylinders through oriented aggregation, J. Phys. Chem. B 2005, 109, 11548.

11 Penn, R. L., Kinetics of oriented aggregation, J. Phys. Chem. B 2004, 108, 12707

12 Park, J.; Privman, V.; Matijevic, E., Model of formation of monodispersed colloids, J. Phys. Chem. B 2001, 105, 11630

13 Chang, Y.; Teo, J. J.; Zeng, H. C., Formation of colloidal CuO nanocrystallites and their spherical aggregation and reductive transformation to hollow $\mathrm{Cu}_{2} \mathrm{O}$ nanospheres, Langmuir 2005, 21, 1074

14 Xi, G.; Xiong, K.; Zhao, Q.; Zhang, H.; Qian, Y., Nucleation-dissolutionrecrystallization: A new growth mechanism for t-selenium nanotubes, Cryst. Growth Des. 2006, 6, 577 
15 Wells, A. F., Structural Inorganic Chemistry, 5th ed; Oxford University Press: London, 1984.

16 Prabakar, S.; Bumby, C. W.; Tilley, R. D., Liquid-phase synthesis of flowerlike and flake-like titanium disulphide nanostructures, Chem Mater. 2009, 21, 8, 1725

17 Margolin, A.; Popovitz-Biro, R.; Albu-Yaron, A.; Rapoport, L.; Tenne, R., Inorganic fullerene-like nanoparticles of $\mathrm{TiS}_{2}$, Chem. Phys. Lett. 2005, 411, 162. 


\section{Conclusions and Future Work}

In this thesis the synthesis and characterization of nanocrystalline materials by solution phase techniques was investigated. Using these technique nanocrystals of silicon and germanium and nanostructures of titanium disulphide were synthesized. The application of Si and Ge nanocrystals as inorganic fluorescent whitening agents where also studied. Techniques such as HRTEM, SAED, EDX, XRD and FTIR were some of the tools used to characterize the synthesized nanomaterials. Research work concerning the various synthetic techniques is summarized in sections 7.1-7.4. In each section, the highlights and significance of the research work reported in the thesis are concluded. Future work concerning each chapter is then summarized in section 7.5.

\subsection{Silicon Nanocrystals}

In Chapter three the syntheses of silicon nanocrystals by the microemlusion method was investigated. The particle size was controlled by surfactants, reductants and varying precursor concentrations. $\mathrm{C}_{12} \mathrm{E}_{5}$ was found to an effective surfactant to aid in the production of free standing silicon nanocrystals, whilst the reducing agents, both lithium aluminium hydride and lithium triethyl borohydride were found to produce nanocrystals of narrow size distribution. Whilst comparing the two halide precursor's silicon tetrachloride and silicon tetrabromide, it was found that the former produced smaller nanocrystals. The optical spectroscopy further confirmed the highly monodisperse nature of the nanocrystals by their narrow room temperature fluorescence spectra. 
In the literature, under bench-top conditions, similar microemlusion reactions have

shown to yield crystalline silicon nanocrystals. ${ }^{1-4}$ However the nanocrystal particle size could not be fine controlled, nor manipulated using reaction conditions. This is also the first example of particle size control using various reductants, surfactants and precursors in the literature pertaining to silicon nanocrystals. Further, studies on improving the yield of the product obtained must be conducted in detailed to facilitate the large scale synthesis using this methodology.

\subsection{Germanium Nanocrystals}

The results presented in chapter four of the thesis represent a good basis to understand the properties of germanium nanocrystals. They also give a solution for the synthesis of monodisperse and size controlled nanocrystals with possible applications in bioimaging. Hydride reducing agents were used to control the particle size of the germanium nanocrystals and, are the first example of its kind in the literature. Smaller nanocrystals were obtained using a strong reductant such as $\mathrm{LiAlH}_{4}$ and larger nanocrystals when using a weak reducing agent such as $\mathrm{NaBH}_{4}$. The UV-Vis absorption and PL spectra of the nanocrystals do not change with solvents or surface termination, indicating that the nanocrystalline core is largely responsible and those solvent or surface effects do not contribute significantly. Emission across a relatively narrow region of $420-480 \mathrm{~nm}$ is observed with full width maximums (FWHM) of 70-80 nm indicating that the germanium nanocrystals are monodisperse. Toxicity studies on the nanocrystals showed that they could be used at concentrations of $100 \mu \mathrm{g} / \mathrm{ml}$ and have relatively low toxicity. The high temperature reduction experiments demonstrated that crystalline 
nanoparticles could be formed although without much size control or the ability to terminate the surface. The current methodology leaves much to be desired in terms of surface modification and size control and hence better synthetic protocols need to be adopted.

\subsection{Fluorescent Whitening Agents}

Silicon and germanium nanocrystals with both amine and alkene surface capping were used as whitening agents for woolen fabric. Strong blue luminescence was observed for both the nanocrystals when applied to the fabric and was found to improve its brightness, compared to commercial FWA, Uvitex. Silicon-amine and silicon-alkene treated fabrics were found to have increased stability against both UVA and UVB radiation, when applied at low concentrations. Under UVA irradiation, LUM was observed to be degraded in the presence of both Uvitex and amine-capped nanoparticles at high concentration. Over $12 \mathrm{~h}$ UVA, $59 \%$ of the LUM degraded to APA in the presence of Uvitex, while over the same time period, only $45 \%$ and $26 \%$ LUM $\rightarrow$ APA degradation was observed for Si-amine and Ge-amine nanoparticles, respectively. This reflects reduced generation of singlet oxygen by the nanoparticles, compared to the organic FWA, Uvitex, particularly in the case of the Ge-amine nanoparticles. Reduced reactive oxygen species generation is a desirable attribute for a new generation of whitening and brightening agents. 


\subsection{Titanium Disulphide}

Nanostructures of titanium disulphide were synthesized by solution phase routes in both coordinating and non-coordinating solvents.

A new method was successfully developed to synthesize highly crystalline titanium disulphide nanostructures in solution. Flower-like and flake-like nanostructures were produced by varying the injection temperature of the titanium source into the 1Octadecene/sulphur solution. Two different pathways were suggested for the formation of the final products; an instant nucleation to form $\mathrm{TiS}_{2}$ flakes and spherical nuclei to form flower-like nanostructures. The flower-like nanostructures were found to have higher BET surface area compared to the flake-like nanostructures and previously reported surface areas for analogous $\mathrm{TiS}_{2}$ nanostructures. Whilst using oleylamine as solvent, the low temperature injection yielded hollow spheres of $\mathrm{TiS}_{2}$ and the high temperature injection, fullerene-like nanoparticles of $\mathrm{TiS}_{2}$. The property of oleylamine to selectively bind to the nanostructure surface in conjunction with the effect of injection temperature was understood to be behind the growth of these nanostructures. 


\subsection{Future Work:}

The results discussed in chapter 3 and 4 present a good basis to understand the synthesis and properties of silicon and germanium nanocrystals in solution. The current synthesis may be used to prepare a range of $\mathrm{Si}$ and Ge nanocrystals that are monodisperse and non-oxidized. Future work on the current synthetic methodology would be to investigate the method for the production of large-scale and monodisperse Si and Ge nanocrystals with a wider range of sizes (colors). This could be made possible by using the same methodology, but parameters such as the amount of precursor, surfactant type and reductant should be varied to achieve this. The production of bigger nanocrystals would provide a wider range of applications, especially in bio-imaging, where organic dyes emitting $<600 \mathrm{~nm}$ are problematic, since they tend to damage biomaterials by the UV light required to excite it. Further the use of $\mathrm{Si}$ and Ge nanocrystals for bio-imaging purposes should be extended to a wider variety of cell types, as each of them might have different cell toxicities. ${ }^{5}$

The synthesis of $\mathrm{Si} / \mathrm{Ge}$, core/shell or alloy nanoparticles by solution-phase routes are very few and limited. ${ }^{6}$ These nanostructures are known to have combined potential applications in potential applications in thermo-photovoltaics (TPV) and solar cells, as

well as in silicon based optoelectronic devices. ${ }^{7-8}$ Using the current methodology, the synthesis of either core/shell or alloy nanocrystals of Si and Ge could be synthesized. By co-reducing, both $\mathrm{SiCl}_{4}$ and $\mathrm{GeCl}_{4}$ and modifying the surface with suitable capping agents, it could be possible to alloy or make core/shell nanocrystals. Similarly the 
doping of these nanocrystals with atoms such as manganese ${ }^{9}$ could be investigated by this synthetic methodology, because of their applications as bi-functional (paramagneticoptical) nanomaterials.

The results described in chapter 5, illustrate the applications of Si and Ge nanocrystals as fluorescent whitening agents. The plausibility of directly attaching the nanocrystals to the surface of the wool through chemical interactions should be investigated. The chemically rich wool surface offers a plethora of ways by which a functionalized Si and Ge nanocrystal could be attached. The use of coatings could be avoided and further help in up-scaling the current synthetic methodology.

The synthesis of titanium disulphide nanostructures by solution techniques is detailed in chapter 6 . The successful synthesis of stoichiometric $\mathrm{TiS}_{2}$ provides a background for the synthesis of other metal dichalcogenide systems. More research is needed to refine the methods presented in this chapter, in order to control the size and morphology of the titanium disulphide nanostructures. The current method allows the synthesis of 3D flower-like nanostructures and 2D flake-like nanostructures using non-coordinating solvents. Further, hollow spheres and fullerene-like nanoparticles are obtained using coordinating solvents. Though these nanostructures are interesting in their own right, the synthesis of much smaller structures could result in more interesting properties, due to quantum confinement effects. To fine-tune the synthetic methodology, an in-depth study of the reaction kinetics is needed. Reaction temperature, amount of precursors and growth temperature are some parameters that could be studied to bring about this effect. 
Its is well known that layered metal di-chalcogenides such as $\mathrm{TiS}_{2}$ and $\mathrm{MoS}_{2}$ are of great interest as host lattices, by reacting with a variety of guest atoms $(\mathrm{Li}, \mathrm{Na})$, to yield intercalation compounds. It would be interesting to investigate if $\mathrm{TiS}_{2}$ nanostructures synthesized by this method could be intercalated in solution by guest atoms such as $\mathrm{Li}$ and Na. Further work on extending the synthesis to other metal-dichalcogenide systems such as $\mathrm{MoS}_{2}, \mathrm{NbS}_{2}$ and $\mathrm{ZrS}_{2}$ should also be investigated. 


\subsection{References:}

1. Rosso-Vasic, M., Sprujit, E., Lagen, B. V., Cola, L. D., Zuilhof, H, Alkylfunctionalized oxide-free Silicon Nanoparticles: Synthesis and Optical Properties, Small, 2008, 10, 1835-1841.

2. Ruizendaal, L.; Bhattacharjee, S.; Pournazari, K.; Rosso-Vasic, M., de Haan, L. H. J.; Alink, G. M.; Marcelis, A. T. M.; Zuilhof, H, Synthesis and cytotoxicity of silicon nanoparticles with covalently attached organic monolayers, Nanotoxicology, 2009, 3,4, 339-347.

3. Rosso-Vasic, M., Sprujit, E., Popovi, Z.; Overgaag, K.; Lagen, B. V.; Grandidier, B.; Vanmaekelbergh, D.; Domínguez-Gutiérrez, D.; Cola, L. D., Zuilhof, H, Amine-terminated silicon nanoparticles: synthesis, optical properties and their use in bio-imaging, J. Mater. Chem, 2009, 19, 5926.

4. Bee, T. G.; Saravanan, N.; Tilley, R, D.; Ramesh, S.; Lim, Y. S. Colloidal synthesis of silicon nanocrystals via inverse micelles microemlusion. Zeitschrift fuer Physikalische Chemie, 2009, 223, 12, 1417-1426.

5. Li, Z.; Zhang, Y.; Jiang, S, Multicolor core/shell-structured up-conversion fluorescent nanoparticles. Adv. Mater, 2008, 20, 4765-4769.

6. Yang, C-S.; Kauzlarich, S. M.; Synthesis and characterization of Ge /Si/alkyl and Ge/silica core-shell quantum dots. Chem. Mater, 1999, 11(12), 3666-3670. 
7. Palfinger, G.; Bitnar, B.; Sigg, H.; Muller, E.; Stutz, S.; Grutzmacher, D. Absorption measurement of strained SiGe nanostructures deposited by UHVCVD, Physica E, 2003, 16, 48.

8. Yang, Y. M.; Wu, X. L.; Siu, G. G.; Huang, G. S.; Shen, J. C.; Hu, D. S. Formation, structure, and phonon confinement effects of nanocrystalline $\mathrm{Si}_{1-\mathrm{x}} \mathrm{Ge}_{\mathrm{x}}$ in $\mathrm{SiO}_{2}-\mathrm{Si}$-Ge co-sputtered films, J.Appl.Phys. 2004,96(9)

9. Zhang, Z.; Brynda, M.; Britt, D.; Carroll, E.; Larsen, D. S.; Louie, A. Y.; Kauzlarich, S. M.; Synthesis and characterization of manganese doped silicon nanoparticles, J. Am. Chem. Soc. 2007, 129(35), 10668-10669. 